\title{
WestVirginiaUniversity
}

THE RESEARCH REPOSITORY @ WVU

Graduate Theses, Dissertations, and Problem Reports

2005

\section{fMRI studies of amblyopia: Pediatric and adult perspectives}

\author{
Ian Patrick Conner \\ West Virginia University
}

Follow this and additional works at: https://researchrepository.wvu.edu/etd

\section{Recommended Citation}

Conner, lan Patrick, "fMRI studies of amblyopia: Pediatric and adult perspectives" (2005). Graduate Theses, Dissertations, and Problem Reports. 2350.

https://researchrepository.wvu.edu/etd/2350

This Dissertation is protected by copyright and/or related rights. It has been brought to you by the The Research Repository @ WVU with permission from the rights-holder(s). You are free to use this Dissertation in any way that is permitted by the copyright and related rights legislation that applies to your use. For other uses you must obtain permission from the rights-holder(s) directly, unless additional rights are indicated by a Creative Commons license in the record and/ or on the work itself. This Dissertation has been accepted for inclusion in WVU Graduate Theses, Dissertations, and Problem Reports collection by an authorized administrator of The Research Repository @ WVU.

For more information, please contact researchrepository@mail.wvu.edu. 


\title{
fMRI Studies of Amblyopia: Pediatric and Adult Perspectives
}

\author{
Ian Patrick Conner
}

\author{
Dissertation submitted to the \\ School of Medicine \\ at West Virginia University \\ in partial fulfillment of the requirements \\ for the degree of
}
Doctor of Philosophy
in

Neurobiology and Anatomy

\author{
Janine D. Mendola, Ph.D., Chair \\ Ariel Agmon, Ph.D. \\ Susan K. Lemieux, Ph.D. \\ J. Vernon Odom, Ph.D. \\ Adrienne K. Salm, Ph.D.
}

\author{
Department of Neurobiology and Anatomy \\ Morgantown, West Virginia \\ 2005
}

Keywords: Amblyopia, Vision, fMRI, Children 


\section{Abstract \\ fMRI Studies of Amblyopia: Pediatric and Adult Perspectives}

\section{Ian Patrick Conner}

Functional magnetic resonance imaging (fMRI) is currently the technique of choice for mapping functional neuroanatomy in humans, and over the past 15 years there has been a dramatic growth in the number of studies that provide brain-behavior correlations in normal healthy adults. More recently, a few studies have begun to make such measures in healthy children. In addition, fMRI is increasingly being applied to study brain function in subjects with neurological disease. The overall aim of these studies was to apply fMRI methods to the study of amblyopia, the most prevalent developmental vision disorder. Amblyopia develops early in life, usually before 5 years old, and is most treatable during childhood. Our approach was to study both children and adults with either the strabismic or the anisometropic type of amblyopia. In our first experiment (Chapter 3), we applied fMRI techniques to map retinotopic visual organization in children. We conclude that cortical visual organization is measurable and highly mature in children aged 9 to 12 years. In our second experiment (Chapter 4), we applied similar techniques to adults with amblyopia. We conclude that visual field organization is abnormal in the brains of these adults. In our final experiment (Chapter 5), we applied these same techniques to children with amblyopia, and observed abnormalities similar to those seen in adults. These studies present a novel neurological characterization of amblyopia, and provide a basis for further studies of human visual development, in health and disease. 


\section{Dedication}

My wife, Toni, has provided immeasurable support and encouragement throughout the process of completing this work. She inspires me and keeps me sane, and I feel so fortunate to have found such a wonderful and beautiful partner in life. I dedicate this work to her.

In addition, I must also acknowledge the loving support of my parents, Gary and Carolyn Conner, and my wife's parents, Jim and Marietta Hartline. They have been constant cheerleaders who never seem to tire of listening to stories of my successes and failures. Despite the fact that I never seem to stop "going to school," they have always been there with words of encouragement. I could not imagine doing this without them. 


\section{Acknowledgements}

Of course, I must first thank my thesis advisor, Janine. I may never find the words to express my gratitude to her for taking me on as her first student, and for working tirelessly and patiently to mold me into a scientist. I know that I have sometimes tested her, and I have often stubbornly resisted her efforts to help me and improve my work, but I feel blessed to have had her as a mentor. Thank you, Janine. I hope to make you proud.

Likewise, I would like to thank the members of my committee for their contributions and insights. I chose each member carefully to fill a specific advisory role, and I appreciate their efforts as I have worked through this project. I also thank Terry Schwartz, M.D., for her intellectual contributions to helping me learn about and understand childhood vision and amblyopia.

I would also like to thank James Trimmier and Joshua Robinson, the talented computer programmers I have worked with in the lab. I learned a lot from our interactions, and always enjoyed our collaborations. Likewise, I am glad to have worked with and formed friendships with the other students who have come and gone from the lab in my time there, especially Saloni Sharma, Ritwick Agrawal, and Amol Bahekar. I hope to be lucky enough to work with each of you again.

Finally, I would like to thank my friend, Ruth Walsh. Ruth started as a research assistant in the lab a few months after I began, and left for another position last month. Despite our (quite) different social, political, and medical perspectives, I thoroughly enjoyed every conversation and debate through the years. In addition, Ruth did all of the leg work to recruit and maintain our database of volunteers, saving me a tremendous amount of work. Thank you, Ruth.

Finally (finally), thanks to all of my research volunteers, and to all volunteers for medical research everywhere. Everybody has different motivations for helping out with these experiments, but the bottom line is that this work couldn't be done without their help. I respect and appreciate anyone who is willing to give their time, talents, and effort to improving the human condition. 


\section{Table of Contents}

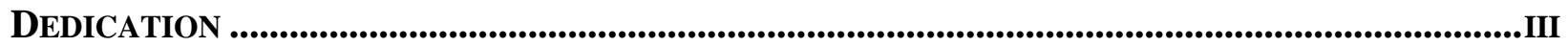

ACKNOWLEDGEMENTS........................................................................................................................... IV

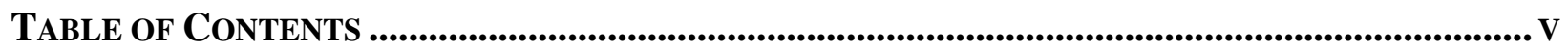

LIST OF FIGURES ….......................................................................................................................

LIST OF ABBREVIATIONS...........................................................................................................................XI

CHAPTER 1: INTRODUCTION AND LITERATURE REVIEW ........................................................... 1

1.1 Overview of the Human Visual System ........................................................................ 2

1.1.1 Anatomical Organization ............................................................................ 2

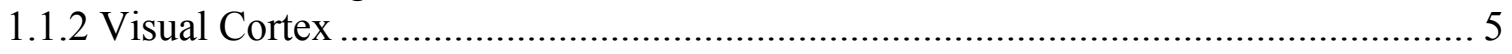

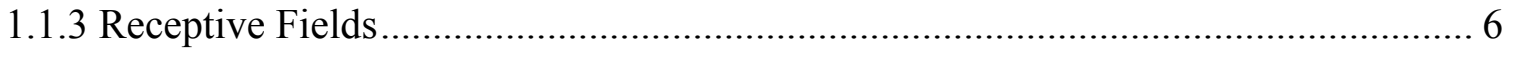

1.2 Normal Development of Spatial Vision............................................................................ 8

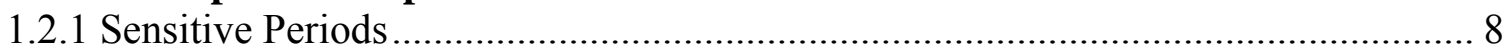

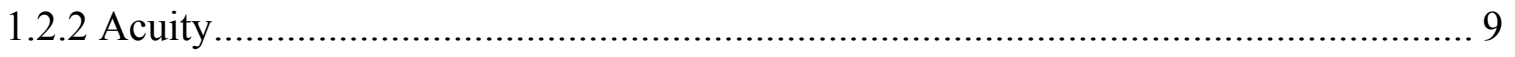

1.2.3 Contrast Sensitivity ………………………………....................................... 9

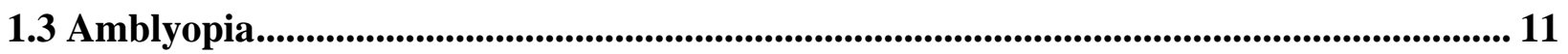

1.3.1 Clinical Description and Significance ………….................................................. 11

1.3.2 Spectrum of Known Visual Deficits .................................................................. 12

1.3.3 Animal Models of Amblyopia …………………….............................................. 13

1.4 Functional Magnetic Resonance Imaging.............................................................................. 15

1.4.1 fMRI and the BOLD Response................................................................. 15

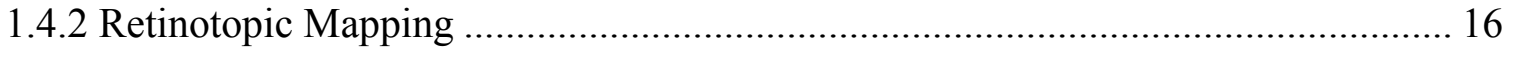

1.5 Functional Imaging of Amblyopia.............................................................................................. 17

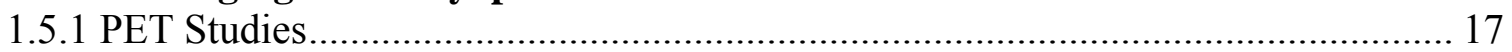

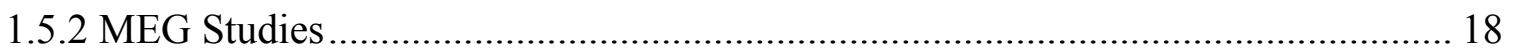

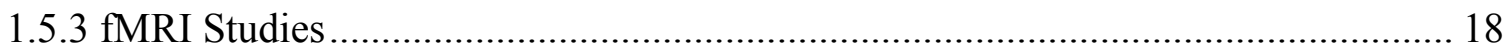

1.6 Figures............................................................................................................................................ 23

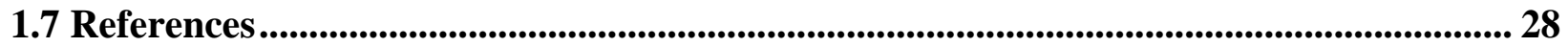

CHAPTER 2: OBJECTIVES AND RATIONALES ............................................................................. 42

2.1 Objective 1- To determine the cortical retinotopic organization in children with normal

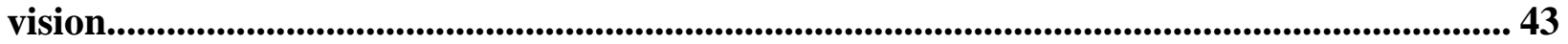

2.2 Objective 2- To determine the cortical retinotopic organization in adults with amblyopia.. 
2.3 Objective 3- To determine the cortical retinotopic organization in children with amblyopia.

CHAPTER 3: RETINOTOPIC ORGANIZATION IN CHILDREN MEASURED WITH FMRI............. 45

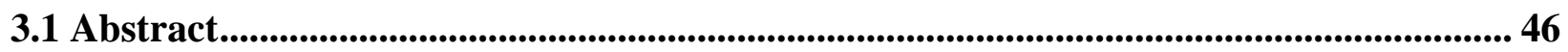

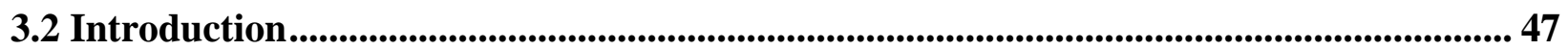

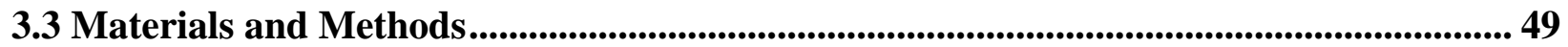

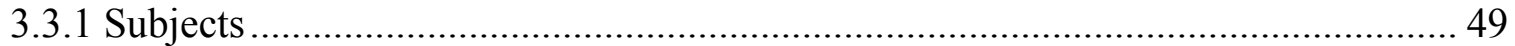

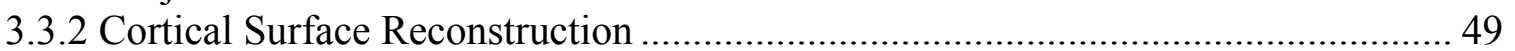

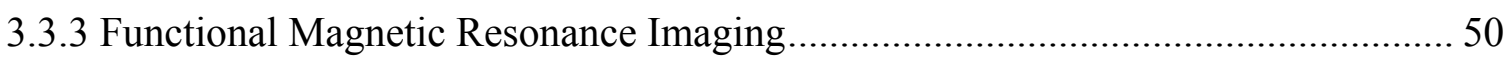

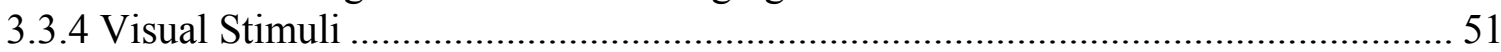

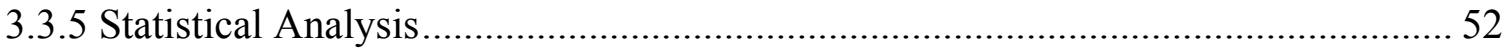

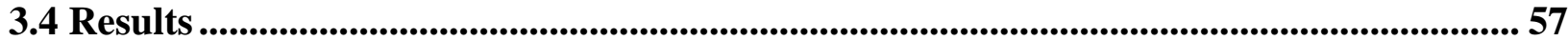

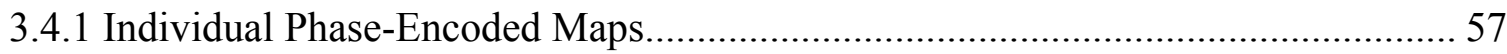

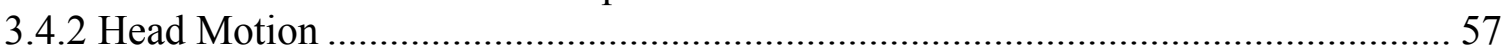

3.4.3 Region-of-Interest Analysis for Across-Group Comparisons................................ 58

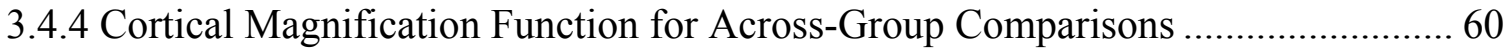

3.4.5 Cortical Surface Averaging for Across-Group Comparisons ................................ 61

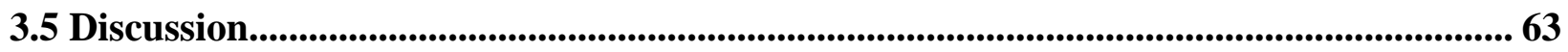

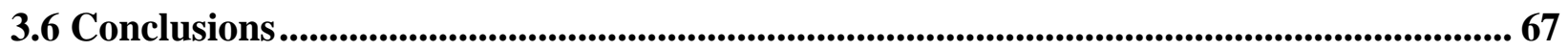

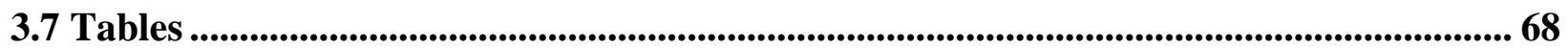

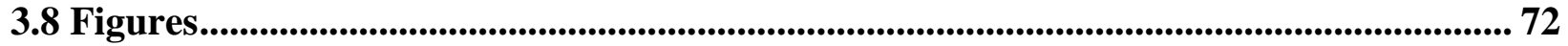

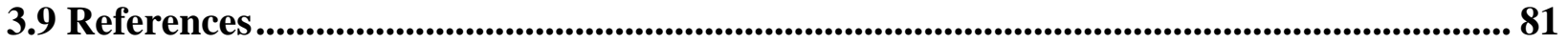

CHAPTER 4: Monocular Representation in Visual CoRTEX OF AMblyopic Adults

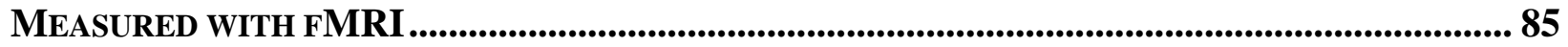

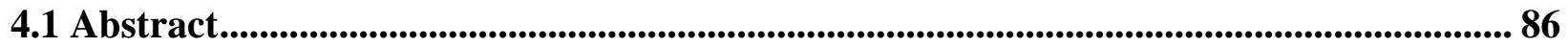

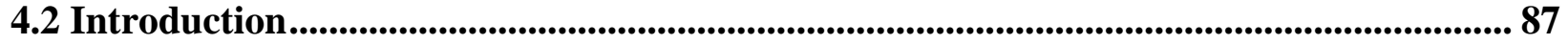

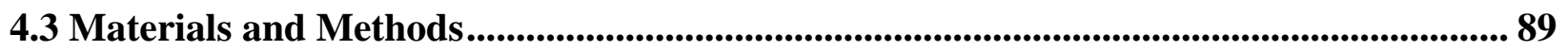

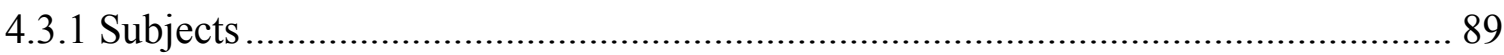

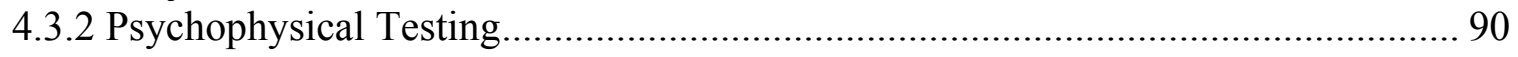

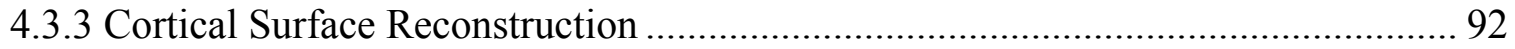

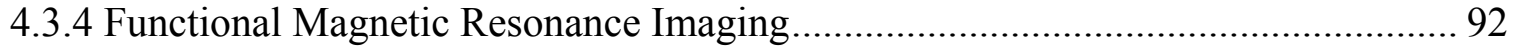

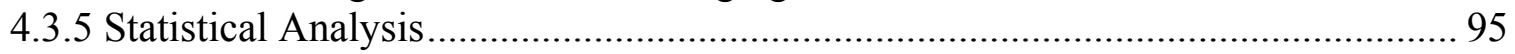

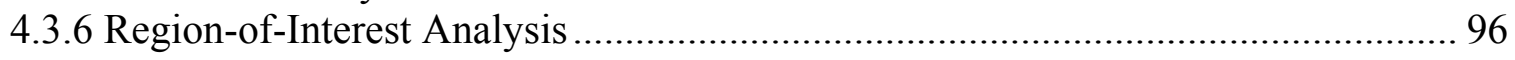

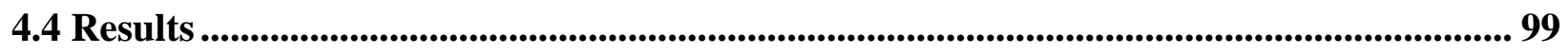

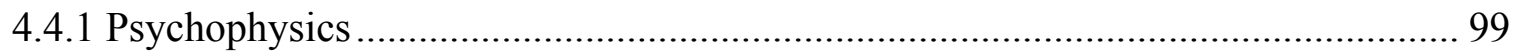

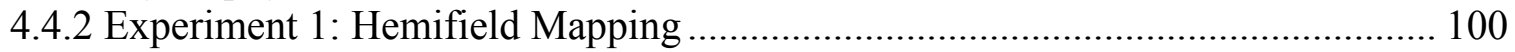

4.4.3 Experiment 2: Eccentricity Mapping ....................................................... 102

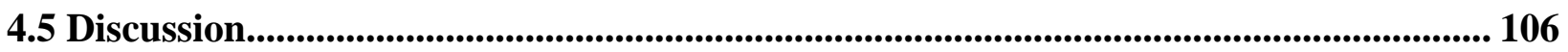




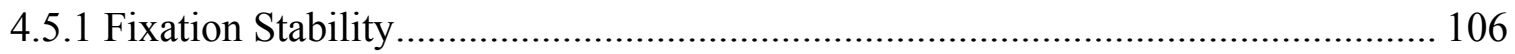

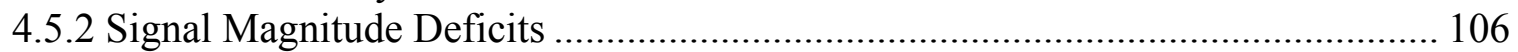

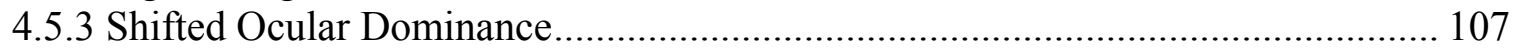

4.5.4 Impaired Cortical Binocularity ........................................................................ 107

4.5.5 Nasotemporal Asymmetry ............................................................................. 108

4.5.6 Visual Field Remapping ………………………....................................... 108

4.5.7 Findings in Temporal and Parietal Cortex …………............................................ 109

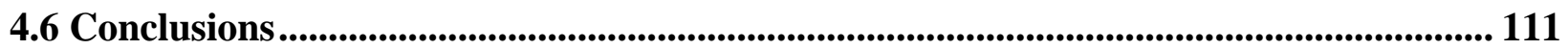

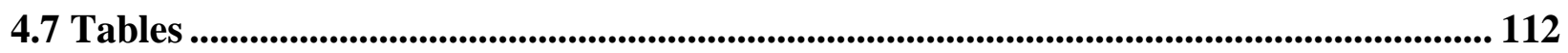

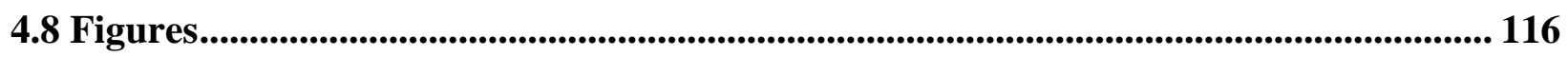

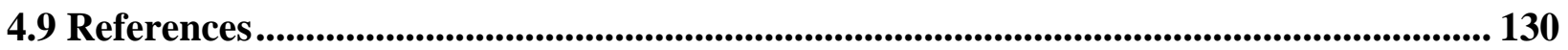

CHAPTER 5: MONOCULAR REPRESENTATION IN VISUAL CORTEX OF AMBLyOPIC CHILDREN

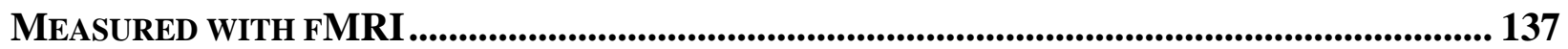

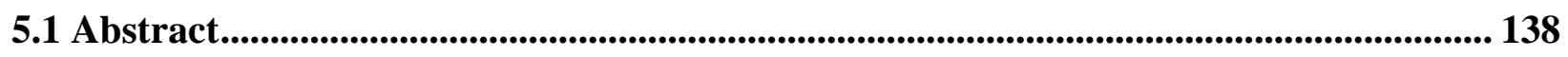

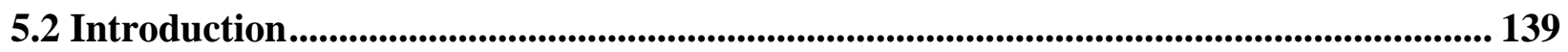

5.3 Materials and Methods................................................................................................ 143

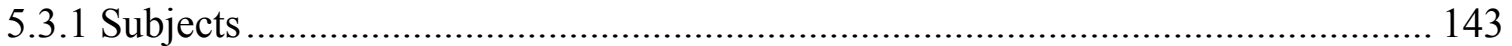

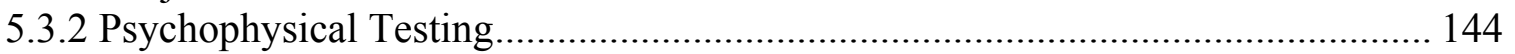

5.3.3 Cortical Surface Reconstruction ...................................................................... 145

5.3.4 Functional Magnetic Resonance Imaging.......................................................... 145

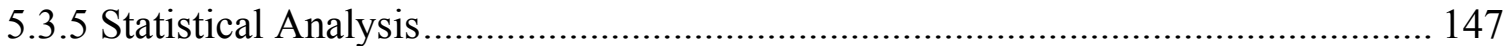

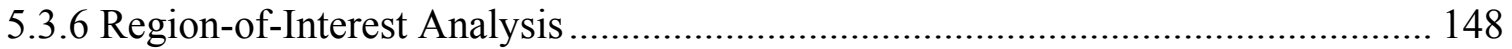

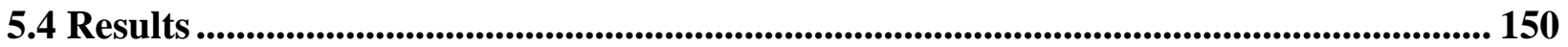

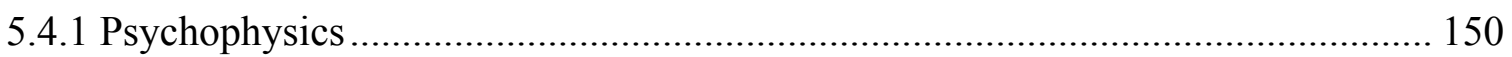

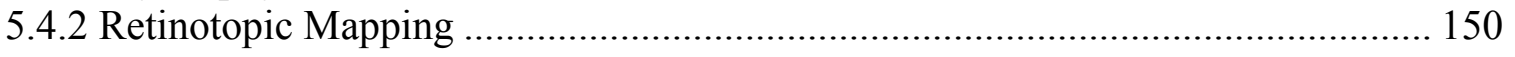

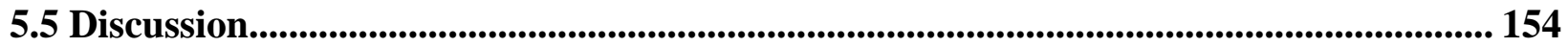

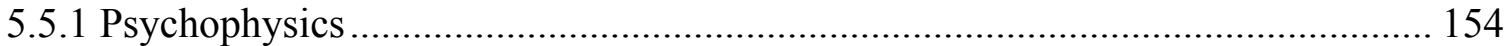

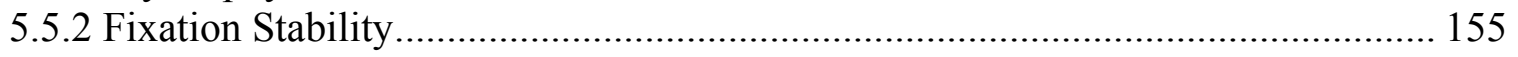

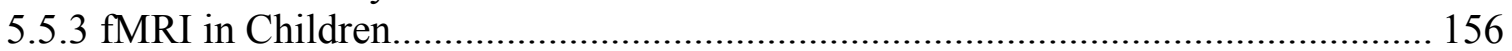

5.5.4 Visual Field Remapping ………………………….................................... 157

5.5.5 Implications for Treating Amblyopia ………………....................................... 158

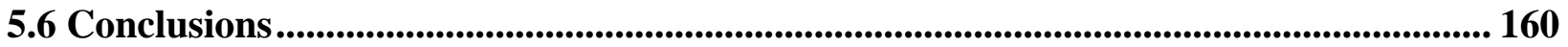

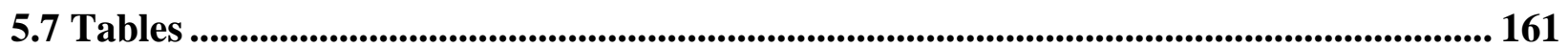

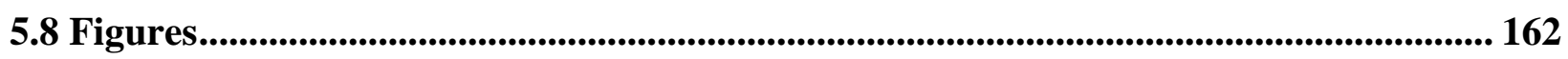

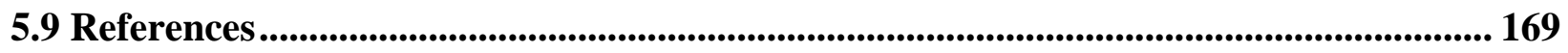

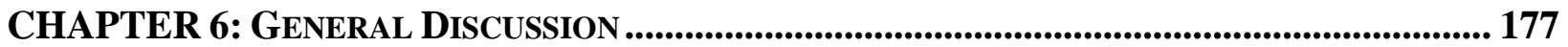

6.1 Summary and Significance of the Current Project ................................................................... 178

6.2 Comparisons with Previous Studies...................................................................................... 182 


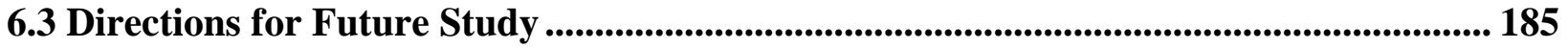

6.4 Conclusions ........................................................................................................................... 186

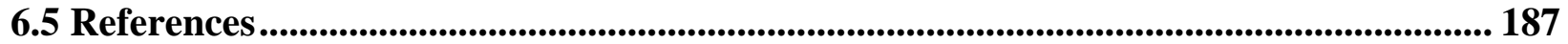

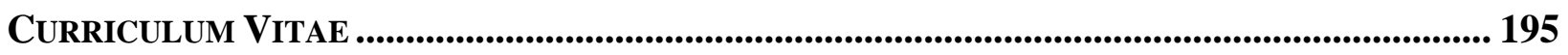




\section{List of Figures}

\section{CHAPTER 1}

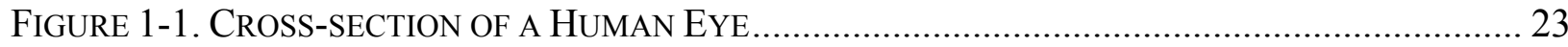

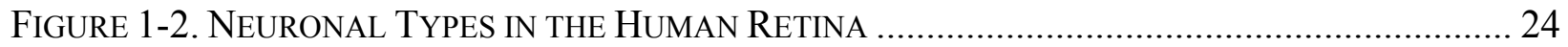

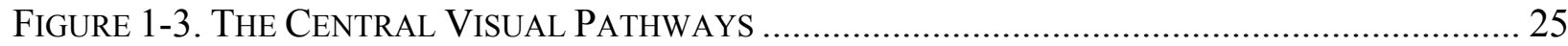

FiguRE 1-4. MulTIPLE ReTINOTOPIC AREAS IN VISUAL CORTEX ………..................................... 26

Figure 1-5. The Contrast Sensitivity FunCtion (CSF) HAS AN INVERTEd U-ShaPe ............. 27

\section{CHAPTER 3}

Figure 3-1. DePiction of DyNAMic EcCENTRICITY AND Polar ANGLE STIMULI ........................ 72

FiguRE 3-2. RETINOTOPIC MAPPING RESUlTS FROM ONE CHILD .................................................... 73

Figure 3-3. Comparison of AVERAge Head Motion During the fMri SCANS for Adults

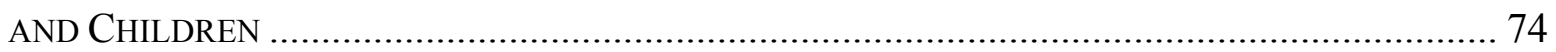

FiguRE 3-4. COMPARISON OF SIZE OF VisUAL AREAS IN CHILDREN AND ADULTS .......................... 75

Figure 3-5. COMPARISON OF CORTICAL MAGNifiCATION FUnCTIONS IN AREA V1 FOR CHILDREN

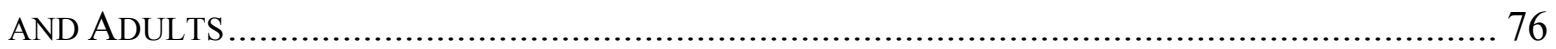

FiguRE 3-6. COMPARISON OF CORTICAL MAGNIFICATION FUNCTIONS IN AREAS V2V AND V2D FOR

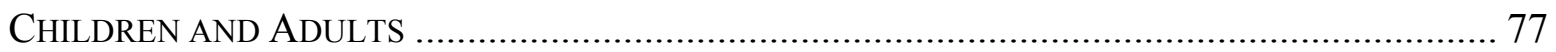

Figure 3-7. COMPARISON OF ECCENTRICITY Signals IN LATERAL OCCIPITO-TEMPORAL CORTEX FOR ADULTS AND CHILDREN …………................................................................ 78

Figure 3-8. Comparison of Polar ANGle Signals in Lateral OCCIPITO-TeMPORAL CorteX FOR ADULTS AND CHILDREN …………………………....................................... 79

Figure 3-9. COMPARISON OF ECCENTRICITY AND POLAR ANGLE Signals In VENTRAL TEMPORAL

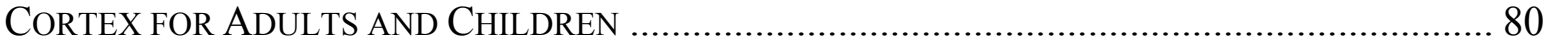

\section{CHAPTER 4}

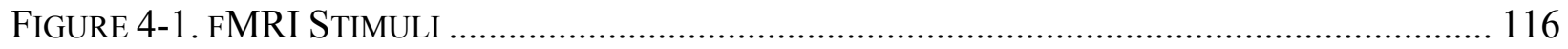

FIGURE 4-2. FIXATION STABILITY FOR EXPERIMENT 1 ……................................................. 117

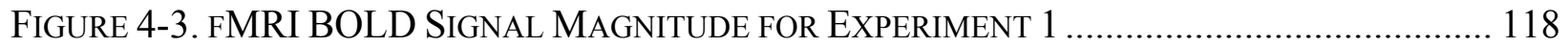

FIGURE 4-4. OCULAR DOMINANCE INDEX BY GROUP AND ROI............................................. 119

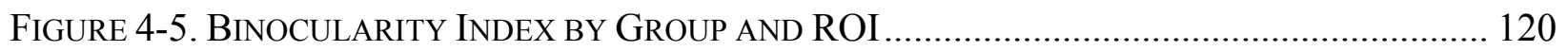

FIGURE 4-6. FIXATION STABILITY FOR EXPERIMENT 2 ……................................................. 121

FigURE 4-7. ECCENTRICITY MAPS FROM AMBLYOPIC PATIENTS .................................................. 123

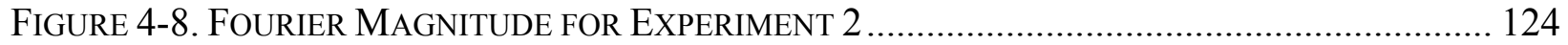

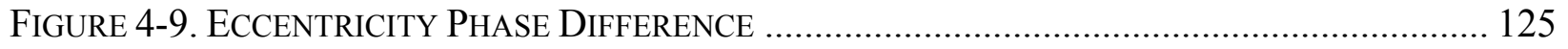

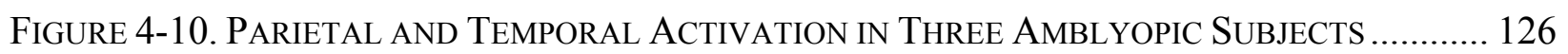

FigURE 4-S1. ECCENTRICITY PHASE CALCULATION FOR EXPERIMENT 2 .................................. 127

FIGURE 4-S2. RESULTS FROM PSYCHOPHYSICS TESTING ……............................................... 128

FigURE 4-S3. ECCENTRICITY MAPS FROM CONTROL PATIENTS....................................................... 129

\section{CHAPTER 5}

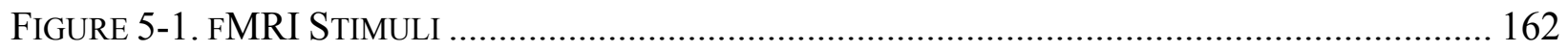

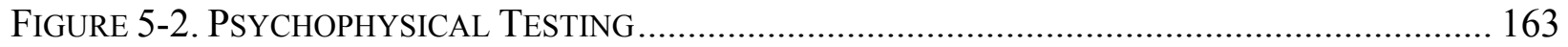

FigURE 5-3. FiXATION STABILITY AND RESPONSE ACCURACY ..................................................... 164 
Figure 5-4. ECCENTRICITY MAPS FROM SiX AMBLYOPIC AND THREE CONTROL CHILDREN ...... 165

Figure 5-5. SignifiCANCE MAPS FROM SiX AMBLyopiC AND ThreE CONTROL CHILDREN ....... 166

Figure 5-6. Fourier Signal Magnitude is DePRESSED for AMBLyopic Eye Viewing......... 167

Figure 5-7. ECCENTRICITY PHASE IS SHIFTED FOR AMBLYOPIC SUBJECTS .............................. 168 


\section{List of Abbreviations}

$\begin{array}{ll}\text { AE } & \text { amblyopic eye } \\ \text { ANISO } & \text { anisometropic amblyopia } \\ \text { BOLD } & \text { blood oxygen level dependent } \\ \text { BI } & \text { binocularity index } \\ \text { CO } & \text { cytochrome oxidase } \\ \text { cpd } & \text { cycles per degree } \\ \text { CRT } & \text { cathode ray tube } \\ \text { CSF } & \text { contrast sensitivity function } \\ \text { CTL } & \text { control } \\ \text { DE } & \text { dominant eye } \\ \text { DI } & \text { dominance index } \\ \text { deg } & \text { degrees } \\ \text { EEG } & \text { electroencephalography } \\ \text { ExFOV } & \text { extrafoveal calcarine ROI } \\ \text { FE } & \text { fellow eye } \\ \text { FG } & \text { fusiform gyrus } \\ \text { fMRI } & \text { functional magnetic resonance imaging } \\ \text { FOV } & \text { foveal ROI } \\ \text { IPS } & \text { intra-parietal sulcus } \\ \text { LCD } & \text { liquid crystal display } \\ \text { LGN } & \text { lateral geniculate nucleus } \\ \text { MAR } & \text { minimum angle of resolution } \\ \text { MEG } & \text { magnetoencephalography } \\ \text { MRI } & \text { magnetic resonance imaging } \\ \text { MT } & \text { middle temporal visual area } \\ \text { MTG } & \text { middle temporal gyrus } \\ \text { NDE } & \text { non-dominant eye } \\ \text { OD } & \text { oculus dexter, the right eye } \\ \text { OS } & \text { oculus sinister, the left eye } \\ \text { PET } & \text { positron emission tomography } \\ \text { RGC } & \text { retinal ganglion cell } \\ \text { ROI } & \text { region-of-interest } \\ \text { SF } & \text { spatial frequency } \\ \text { SPECT } & \text { single photon emission computed tomography } \\ \text { STRAB } & \text { strabismic amblyopia } \\ \text { VA } & \text { visual angle } \\ \text { VBM } & \text { voxel-based morphometry } \\ \text { VEP } & \text { visual evoked potential } \\ \text { V1 } & \text { primary/striate visual area } \\ \text { V1 } & \text { extrafoveal V1 region-of-interest } \\ \text { V2 } & \text { secondary visual area } \\ \text { V2 } & \text { extrafoveal V2 region-of-interest } \\ & \end{array}$


CHAPTER 1: Introduction and Literature Review 


\subsection{Overview of the Human Visual System}

Human vision is the process of transforming photons of light into coherent perception. It occurs seemingly without effort, yet all attempts to reproduce even the most fundamental visual functions in machines have proven exceedingly difficult. Consider, for a moment, the complexity of the system. Light must first be transduced into an electrical signal at the retina by specialized neurons called photoreceptors. This signal is processed in the retina, transmitted through at least one relay to the visual cortex, where it is further processed and distributed to multiple visual areas in the brain for integration with information from other neural processes. We then use this information to make decisions- jump or crouch, eat or don't eat, friend or foe. We also use this information to navigate in space, determining distance, direction, and depth, and avoiding danger. We read, we drive, we create and appreciate art, and we take all of these things for granted, because of the seeming simplicity of visual perception. It is a remarkable system.

However, the "machine" of human vision is susceptible to failure, particularly when its normal postnatal development is interrupted. Since human vision requires experience for proper development, the first months and years of life are crucial. Impaired visual function and perception result from abnormal development, and are largely untreatable in adults after development is complete. This work aims to better understand the consequences of this process, ultimately so that better management and treatment of patients with impaired vision can be achieved.

First, though, it is necessary to have some background information about the organization and physiological properties of the normal human visual system. This section will cover some of the basic concepts necessary to understand abnormal visual development and perception. It will begin with a brief description of the anatomical organization of the visual system, placing particular emphasis on the central visual pathways. This will be followed by a discussion of the organizing principles in visual cortex, and the concept and properties of visual receptive fields.

\subsubsection{Anatomical Organization}

The eye is a complex sensory organ comprised of optical and neural elements, which develops from the neural tube as an outgrowth of the prosencephalon, and therefore is truly a part of the central nervous system (Pei and Rhodin, 1970; Barishak, 1992). The optical 
components of the eye serve to refract and focus light onto the retina (Fig. 1-1). Most of the refraction occurs at the cornea, the thick, fibrous, anterior window of the eye. In the normal eye, the elastic lens, by action of the ciliary muscle, refines the focus to form a clear, inverted image on the retina via a process called accommodation. However, eyes which are too long in the anteroposterior dimension are unable to focus far away objects, while eyes which are too short cannot focus close objects. These conditions are termed myopia (i.e., near-sightedness) and hyperopia (i.e., far-sightedness), and are important because they can lead to one type of amblyopia, which will be discussed later (von Noorden and Campos, 2001).

The retina, which lines the entire posterior chamber of the eye, contains the first neural elements of the visual system (Kuffler, 1953). Although the terminology is variable, the sensory retina is composed of ten layers and at least five different classifications of neurons: the photoreceptors and bipolar, ganglion, horizontal, and amacrine cells, in addition to supporting cell types (Fig. 1-2) (Wassle and Boycott, 1991; Dacey, 2000). The photoreceptors are located in the outermost layer of the retina and are comprised of two types: rods and cones (O'Brien, 1982). The rods, distributed throughout the retina except in the fovea (i.e., the central 2 degrees of the retina), are primarily responsive under low luminance levels, and have only one photopigment. In contrast, the cones, concentrated in the fovea, are most responsive under higher luminance levels, and possess one of three different photopigments (Curcio et al., 1987). Both photoreceptor types signal to specialized bipolar cells, which in turn signal to retinal ganglion cells (RGCs). Communication occurs in a basically serial fashion, with pooling and refinement of visual information via the horizontal and amacrine cells, which are types of retinal interneurons (Yamada and Ishikawa, 1965; Dowling and Boycott, 1966). The RGCs, located in the innermost nuclear layer of the retina, project their axons to form the optic nerve, providing the output from the retina to the lateral geniculate nucleus (LGN) of the thalamus, the superior colliculus, and the pretectum of the midbrain. The largest projection is to the LGN, although the other pathways are important for the regulation of eye movements, accommodation, and pupillary constriction. The optic nerve exits the eye at the papilla, or optic disk, where retinal layers are absent and the physiologic blind spot is located.

Specialized RGCs transmit different aspects of the visual image from the retina (Boycott and Wassle, 1974; Kaplan and Shapley, 1986). A class of large RGCs, called parasol or Y cells, responds quickly but transiently to sustained illumination and projects to the ventral two laminae 
of the LGN, termed the magnocellular layers (Leventhal et al., 1981; Conley and Fitzpatrick, 1989). These neurons are insensitive to color but quite sensitive to luminance contrast ( $\sim 2 \%$ or less), and are optimally tuned for low spatial and high temporal frequencies (Hicks et al., 1983; Shapley and Lennie, 1985). A class of smaller RGCs, called midget or X cells, adapts more slowly to specific wavelengths of light and projects to the dorsal four laminae of the LGN, termed the parvocellular layers (Conley and Fitzpatrick, 1989; Rodieck and Watanabe, 1993). These neurons are sensitive to color but relatively insensitive to luminance contrast $(\sim 10 \%)$, and are optimally tuned for high spatial and low temporal frequencies (Schiller and Colby, 1983; Shapley and Lennie, 1985). Approximately $80 \%$ of all RGCs in the human retina are of the midget or X cell type, while parasol or Y cells account for about 10\% (Dacey, 1994). Several other morphologies are also present in lower proportions.

In the LGN, each lamina possesses a retinotopic map of visual space (Erwin et al., 1999). Retinotopy is a key organizing principle of the visual system which maintains ordered "labeled line" connections, analogous to somatotopy in the somatosensory system (Tootell et al., 1982; Tootell et al., 1998). In other words, RGC axons transmitting information about adjacent retinal points project to LGN neurons adjacent to one another. However, as with somatotopy, each half of the brain (at least in early sensory structures) represents only the opposite half of the external world. Retinotopy divides visual space into left and right hemifields, such that axons carrying information from the left half of visual space (i.e., the left nasal retina and right temporal retina) project to the right LGN, and axons carrying information from the right half of visual space (i.e., the right nasal retina and left temporal retina) project to the left LGN (Fig. 1-3) (Stone et al., 1973; Chalupa and Lia, 1991). RGC axons projecting from the contralateral nasal retina cross the midline at the optic chiasm and terminate in laminae 1, 4, and 6, while axons from the ipsilateral temporal retina terminate in laminae 2, 3, and 5 (Wiesel and Hubel, 1966). This eyespecific segregation into layers has been recently confirmed in humans using post-mortem diI fluorescent staining (Hevner, 2000).

Axons originating from LGN neurons project to ipsilateral primary visual cortex in the occipital pole of the brain (Fig. 1-3). This cortical region is also commonly known as V1 or striate cortex, due to the thick stripe of Gennari found in the input layer (layer 4) which can be seen with the naked eye in cross-section. Magnocellular LGN layers project primarily to layer 
4C $\alpha$, while parvocellular LGN neurons project to 4C $\beta$ (Hubel and Wiesel, 1972; Florence and Casagrande, 1987).

Furthermore, the distribution of these axons is divided by the horizontal meridian, with the upper visual field represented below the calcarine sulcus, and the lower visual field above the calcarine sulcus. Visual cortex both above and below the calcarine sulcus is further divided into multiple visual areas, each with its own retinotopic map of visual space.

\subsubsection{Visual Cortex}

The neocortex contains six cellular layers with columnar organization, and is approximately $2 \mathrm{~mm}$ thick in human V1 (Powell and Mountcastle, 1959; O'Kusky and Colonnier, 1982; Mountcastle, 1997). Neurons present include glutamatergic pyramidal and spiny stellate cells, as well as a wide variety of interneurons, which are mostly GABAergic (Lund, 1987; Lund et al., 1988; Lund and Yoshioka, 1991; Lund and Wu, 1997). V1 is organized into orientation and ocular dominance columns, meaning that for a given neuron and its columnar counterparts, there is a preferential bias for stimuli of a particular orientation presented to either the ipsilateral or contralateral eye (Hubel and Wiesel, 1962; Hubel and Wiesel, 1968; Hubel and Wiesel, 1969). Ocular dominance and orientation columns are organized orthogonally to each other across V1, and together form retinotopically distributed hypercolumns (Hubel and Wiesel, 1977). Additional organizational features such as cytochrome oxidase blobs, thought to be important in the parvocellular color pathway, are also found in V1 (Livingstone and Hubel, 1982; Livingstone and Hubel, 1984).

Within V1, there is also an extensive network of long-range horizontal connections. These connections can be excitatory or inhibitory, and seem to be present in all layers, linking cortical columns with similar response properties (McGuire et al., 1991; Hirsch and Gilbert, 1991; Bosking et al., 1997). These connections typically span cortical distances of at least 6-8 $\mathrm{mm}$, a distance much larger than the minimum discharge field size for V1 neurons (Gilbert, 1998). For this reason, it is thought that these connections are likely responsible for shaping receptive field surround effects (see 1.1.3 Receptive Fields), whereby stimulation outside of a neuron's receptive field can modulate its response to stimuli within its receptive field (Kapadia et al., 1995). These connections also provide a potential substrate for cortical plasticity. 
Much has been made of the observation that certain regions within V1 and the neighboring visual area V2 stain heavily for the mitochondrial enzyme cytochrome oxidase (CO) (Horton, 1984; Tootell et al., 1984). Most evidence indicates that magnocellular outputs from layer $4 \mathrm{C} \alpha$ primarily project to layer $4 \mathrm{~B}$ of $\mathrm{V} 1$, and then to the $\mathrm{CO}$ thick stripes of $\mathrm{V} 2$. Outputs from each of these locations can also project directly to other visual areas, including area MT which is known to selectively process information about the movement of visual stimuli (although MT also receives direct geniculate input) (Sincich et al., 2004). In contrast, the parvocellular outputs from layer $4 \mathrm{C} \beta$ project primarily to either the $\mathrm{CO}$ patches or interpatches of layers 2 and 3 in V1. These neurons seem specialized to encode either information about color or form features of visual stimuli (Livingstone and Hubel, 1987). Patch neurons then project to thin $\mathrm{CO}$ stripes in V2, while interpatch neurons project to both pale and thick stripes, merging the magnocellular and parvocellular streams (Sincich and Horton, 2002).

Visual information is transmitted from V1 and V2 to other visual areas as well as to other regions of the brain. These extrastriate visual areas are hierarchically organized and include in humans V2d, V3, and V3A dorsally, and V2v, VP, and V4v ventrally (Van Essen et al., 1992; Van Essen et al., 2001). Each extrastriate cortical area contains another retinotopic map of visual space, processing increasingly more complex stimulus features with increasingly larger receptive fields (Desimone et al., 1985; Maunsell and Newsome, 1987). In addition, there are extensive feedback connections from extrastriate visual areas as well as other parts of the brain to early visual cortex, liking a large network of visually responsive cortical regions. In the macaque, cortex that is exclusively or predominantly visually responsive accounts for about $50 \%$ of the total cortical surface area (Felleman and Van Essen, 1991). In part because of the greatly expanded frontal lobe, this ratio is lower in humans but is still quite substantial (Van Essen et al., 2001). Fig. 1-4 illustrates the organization of several of the known early retinotopic visual areas in the brain (Tootell and Hadjikhani, 2001).

\subsubsection{Receptive Fields}

The receptive field is perhaps the most important physiological principal of sensation (Sherrington, 1906). Every neuron in the visual sensory system, from the retina to the cerebral cortex, has a receptive field tuned for a specific retinal location and set of stimulus properties 
(Hartline, 1938). At the neuronal level, receptive fields can be most basically described as regions of visual space where the presentation or withdrawal of light affects the rate of action potentials (Barlow, 1953). In V1, receptive fields are often termed minimum discharge fields, and average about 2 degrees of visual angle in size (Hubel and Wiesel, 1962). Furthermore, receptive fields are shaped by horizontal connections from neighboring neurons, enabling the visual system to function as a change detector. These connections can span significant cortical distances, modulating neuronal firing responses from as far away as 10 degrees of visual angle, or 6-8 $\mathrm{mm}$ of cortical distance (DeAngelis et al., 1994).

Two examples of receptive fields that have already been discussed are the orientation and ocular preferences of pyramidal cells in V1 columns. Ocular dominance is easiest to understand, since it is based on segregation of inputs from the separate eyes, which come together to synapse on V1 neurons in proportions relatively favoring one or the other eye (Wiesel and Hubel, 1963). Orientation preference, however, is an essentially monocular property entirely dependent on the summation and lateral inhibition of increasingly more complex receptive fields from the retina to the cortex (Hubel and Wiesel, 1962; Eysel et al., 1998; Gillespie et al., 2001). In addition to these static properties, receptive fields can also vary in the temporal domain, allowing visual neurons to be sensitive to fast or slow changes, and even motion (Maunsell and Van Essen, 1983a).

Finally, receptive fields generally become larger and more specific for visual features as they progress to higher levels of the visual system (Reid and Alonso, 1996). While a photoreceptor is responsive only to illumination of a single visual location, neurons in high levels of human visual cortex appear to be responsive to large receptive fields containing highly abstracted visual information, such as motion (Tootell et al., 1995; Culham et al., 1999), a specific object or face (Ungerleider and Haxby, 1994; Allison et al., 1994; Puce et al., 1996), or even to the affect of the face (Allison et al., 2000). As an example of the abstracted processing in higher visual areas, selective lesions to human V2 have been shown to produce little effect on visual acuity (unlike V1 lesions), but instead create increased difficulty for detecting a set of collinear dots in a random dot background (Merigan et al., 1993). In contrast, V4v lesions allow for the feature detection, but make it almost impossible to discriminate the orientation of the collinear dots (Merigan, 1996). These examples simply illustrate the specificity of primate visual areas for processing features of the visual scene. 


\subsection{Normal Development of Spatial Vision}

As one might imagine, it can be exceptionally difficult to study vision in infants, who cannot provide overt feedback or responses. Therefore, a specialized set of methods have been developed for use in young children. Psychophysical investigations typically use forced-choice preferential looking (FPL) with a blinded observer (Fantz, 1965; Atkinson, 2002). FPL takes advantage of the tendency for infants to look at "interesting" stimuli more than stimuli which are of less interest (Dobson et al., 1978). The blinded observer records which of two displays or cards an infant looks at, and after many trials, can determine the limits of the child's vision. However, depending on the function being studied, these procedures can be time-consuming and of poor reliability, since it is difficult to control for inattention in infants (Daw, 1995).

Vision researchers also commonly use electrophysiological techniques like visual evoked potentials (VEPs) and electroretinograms (ERGs) to quantify neural responses to stimuli (Braddick et al., 1986). These techniques generally yield more reliable results, since success is not dependent on sustained attention (Regan, 1989). However, repeated measures suing FPL and electrophysiology do show reasonable agreement. This section will describe the development of several key abilities of spatial vision, as have been determined using FPL and electric potential recordings in children.

\subsubsection{Sensitive Periods}

An important concept for discussion of development is the critical or sensitive period (Lewis and Maurer, 2005). Visual experience is essential for normal brain development (Blakemore, 1976; von Noorden and Crawford, 1979). For over 40 years, early visual deprivation has been associated with abnormalities in adult visual cortex (Wiesel and Hubel, 1963). Furthermore, the specific time course of deprivation is important in directing the pattern of deficits, since different visual functions are acquired at varying times and rates throughout development (Harwerth et al., 1990). The sensitive period for a visual function refers then to the time window for its normal maturation (Harwerth et al., 1986). Amblyopia develops when the sensitive periods for functions of spatial vision are disrupted (Blakemore, 1976; Campos, 1995). Two of the most important of these functions, acuity and contrast sensitivity, will now be discussed with their sensitive periods. 


\subsubsection{Acuity}

Acuity is the most basic function of spatial vision. Optotype acuity is commonly measured clinically, usually in the form of the familiar Snellen letters or "tumbling E" forms of eye charts. Optotype charts provide a reliable, fast measure of visual acuity, but can be confounded by "crowding" effects of adjacent elements in patients with visual impairments, like amblyopia (Stuart and Burian, 1962). Therefore, most vision scientists prefer to use gratings to measure acuity.

At birth, grating acuity is quite poor, approximately 40 times worse than that of adults (Dobson and Teller, 1978). However, it improves very rapidly to 8 times worse by six months, and finally reaches adult-like levels at around 4 to 6 years of age (Mayer and Dobson, 1982; Skoczenski and Norcia, 1999; Maurer and Lewis, 2001). Most of the developmental improvement in grating acuity is thought to be due to maturation of the photoreceptor mosaic in the retina (Banks and Bennett, 1988), although refinement of neural connections and receptive fields in the LGN and cortex certainly also play a role (Jacobs and Blakemore, 1988).

\subsubsection{Contrast Sensitivity}

Contrast sensitivity is defined as the minimum amount of contrast needed to detect the presence of a barely visible grating. More importantly, spatial contrast sensitivity imposes a limit on what information is available to the visual system for further processing. Traditionally, sinusoidal gratings with variable contrasts and spatial frequencies have been used to characterize contrast sensitivity (Campbell and Robson, 1968). Sensitivity, defined as the inverse of the threshold, is determined over a range of spatial frequencies, typically from approximately 0.5 18 cycles per degree (cpd) to plot the contrast sensitivity function (CSF). Humans with normal vision are best at seeing spatial frequencies in the middle of this range, typically between 1 and 4 cpd. A typical CSF is shown in Fig. 1-5 (Campbell, 1983). Note that the peak contrast sensitivity for this subject is centered at $2 \mathrm{cpd}$, and the $\mathrm{x}$-intercept is extrapolated to $50 \mathrm{cpd}$. This is the highest resolvable acuity, and is therefore equivalent to grating acuity.

Like acuity, contrast sensitivity is immature at birth (Norcia et al., 1990). Compared with adults, the newborn visual system requires more contrast and is less sensitive to high spatial 
frequencies (Atkinson et al., 1977). Contrast sensitivity then matures in parallel with acuity, improving to adult-like levels between 6 and 9 years of age (Bradley and Freeman, 1982; Ellemberg et al., 1999; Adams and Courage, 2002). The normal development of contrast sensitivity is primarily attributed to maturation of the retinal photoreceptors (Wilson, 1988). 


\subsection{Amblyopia}

\subsubsection{Clinical Description and Significance}

Amblyopia, often referred to as "lazy eye," is a common developmental disorder of vision, affecting approximately $1-3 \%$ of the population (Flom and Neumaier, 1966; Hillis et al., 1983; Ohlsson et al., 2001; Simons, 2005). It is clinically defined as decreased visual acuity in an otherwise healthy and properly corrected eye (acuities most frequently range from 20/30 to 20/60). Reduced visual acuity in the affected eye usually coexists with normal acuity in the patient's other eye (i.e., the fellow eye).

Amblyopia is often characterized as a disorder of form vision, since the "light sense" mediated by the basic retinal mechanisms appears to be intact and normal (Wald and Burian, 1944; Delint et al., 1998; von Noorden and Campos, 2001; Barrett et al., 2004). Instead, the amblyopic deficit has its basis in the abnormal development of visual areas of the brain in response to atypical early visual experience (Campos, 1995; Kiorpes and Movshon, 1996; Grigg et al., 1996; Hess, 2001; Barrett et al., 2004). Since amblyopia results from abnormal neural development, it is not correctable in adults with refractive lenses alone.

There are many impediments to normal visual experience that can result in amblyopia, but the two most prevalent etiologies are: 1) unequal interocular refractive error or 2) a deviated eye (Cobb and MacDonald, 1978; Vaegan and Taylor, 1979; Daw, 1998). When the early impairment is unbalanced refractive error, the amblyopia is termed anisometropic, whereas amblyopia secondary to ocular deviation is termed strabismic. In some cases, both anisometropia and strabismus can be present concurrently. In addition, an infantile unilateral cataract can result in the comparatively severe condition of deprivation amblyopia (von Noorden and Maumenee, 1968). This condition is less prevalent in humans, but common in animal models of the disorder which utilize the eyelid suture technique (Hess and Howell, 1977; BodisWollner, 1980; Bradley and Freeman, 1981; Abrahamsson and Sjostrand, 1988; McKee et al., 2003). Regardless of the cause, the key to all types of amblyopia is that they are associated with an interruption of normal visual experience early in life, during the critical periods of visual development (Burian, 1967; Thomas, 1978; Hess and Jacobs, 1979; Sireteanu and Fronius, 1990). Thus, studies of the visual abilities of humans with amblyopia provide an opportunity to 
understand how early abnormal sensory experience during neural development can affect adult brain organization and function.

Amblyopia is also clinically significant because of its socioeconomic impact. In addition to the cosmetic and social issues faced by an individual with a deviated eye, the impact of the visual impairment is even farther reaching. Lifelong reduced vision affects peoples' lives in many ways, including scholastic progress, recreational activity, and occupational choice (Chua and Mitchell, 2004). Moreover, it is important to realize that individuals with untreated or undertreated amblyopia have effectively monocular vision throughout life, so loss of the nonamblyopic eye through injury or disease renders them functionally blind. A Finnish study reported that the risk of total blindness in patients with untreated amblyopia is nearly 3 times that of the normal population (Tommila and Tarkkanen, 1981). This increased risk is potentially devastating for a person of working age, and could mean the personal loss of thousands or millions of dollars of unrealized income, as well as the societal cost of providing care and rehabilitation.

\subsubsection{Spectrum of Known Visual Deficits}

Prior investigations of human amblyopia have relied heavily on the use of careful psychophysical techniques (Levi and Harwerth, 1980; Hess et al., 1981). These studies have consistently revealed that affected eyes of anisometropic and strabismic amblyopes exhibit impaired visual acuity and decreased contrast sensitivity, especially at high spatial frequencies (Sireteanu and Fronius, 1981; Hess and Pointer, 1985). Acuity and contrast sensitivity are particularly impaired for central vision, regardless of clinical type (Levi and Klein, 1982; Levi and Klein, 1985). However, additional asymmetric acuity and sensitivity deficits have also been reported for the peripheral visual fields of strabismic amblyopes, with the nasal retina more impaired than the temporal retina for strabismics with inward deviations (i.e., esotropic strabismics) (Flom and Bedell, 1985; Kovacs et al., 2000; Sharma et al., 2000). Furthermore, strabismic eyes usually perform worse than anisometropic eyes for letter and vernier acuity relative to contrast sensitivity, suggesting additional sources of spatial uncertainty in visual processing (Cynader, 1982; Hess, 2001). Subsequent reports have found that spatial discrimination functions are particularly impaired in strabismic amblyopes, including target 
localization, contour integration, and enumeration of briefly presented targets (Wiesel and Hubel, 1963; Dews and Wiesel, 1970; Hubel et al., 1976).

In fact, partial or complete suppression of the amblyopic eye's input by the fellow eye can occur when both eyes are open (Harrad, 1996; Sengpiel and Blakemore, 1996). As a result, stereopsis and depth perception based on binocular disparity cues are almost universally absent, forcing the patient to rely on other depth cues, such as texture, shading, size, perspective, and superposition (Gibson, 1950; Gibson and Flock, 1962). For example, normal texture segmentation has been found in strabismic amblyopes (Mussap and Levi, 1999).

\subsubsection{Animal Models of Amblyopia}

Although anisometropic and strabismic amblyopia seem to represent somewhat different pathological conditions, they also share common characteristics at early stages of the visual system. Both types are thought to have their neural basis in primary visual cortex (V1), the first level of the visual system where input from the left and right eyes meet (Smith, III et al., 1997). Early evidence supporting the cortical pathology came from studying induced deprivation amblyopia in young cats and monkeys, primarily via the lid suture technique (Boothe et al., 1982; Smith, III et al., 1985; Movshon et al., 1987). These animals were found to have grossly normal retinas and lateral geniculate nuclei, but significant alterations in the organization of V1. Specifically, the ocular dominance columns of layer IV (revealed using radiolabeling or cytochrome oxidase staining techniques) were dramatically shifted toward the unsutured eye, a consequence of decreased interocular competition. As a result, very few cortical neurons were able to be effectively driven by the sutured eye, and binocularly responding neurons were only sparsely found throughout all layers of visual cortex (Hubel and Wiesel, 1965; Kiorpes and Boothe, 1981; Crewther and Crewther, 1990).

Subsequent animal studies have used less severe manipulations to achieve analogs of human anisometropic (Crawford and Harwerth, 2004) and strabismic amblyopia (Kiorpes et al., 1998; Fenstemaker et al., 2001), and naturally occurring amblyopia has been also been identified in monkeys (Kiorpes and Boothe, 1981; Horton et al., 1997). Like their lid sutured counterparts, these animals also have physiological deficits, but they are generally much milder (Candy, 2000). Psychophysics in these amblyopic monkeys resembles human results, suggesting that the 
neural basis of human amblyopia is likely to be similar to the foundation of the disorder in cats and monkeys (Kiorpes, 1992).

Nevertheless, this simplistic view must be taken with caution, as cortical ocular dominance shifts have not been found humans with amblyopia. In amblyopes who were monocularly blinded late in life, postmortem investigations using cytochrome oxidase histochemistry have found regular ocular dominance periodicity resembling that seen in humans without amblyopia (Horton and Hedley-Whyte, 1984; Horton et al., 1990; Horton and Stryker, 1993; Horton and Hocking, 1996). Therefore, even though animal models are critical for learning more about the mechanisms of amblyopia, the human condition cannot be expected to exactly parallel these models. Other techniques, such as neuroimaging, must be employed for exploring the anatomical and functional underpinnings of human amblyopia. 


\subsection{Functional Magnetic Resonance Imaging}

\subsection{1 fMRI and the BOLD Response}

Functional magnetic resonance imaging (fMRI) has emerged over the past decade as a powerful, noninvasive technique for studying patterns of neural activation in humans (Menon et al., 1992; Kwong et al., 1992; Ogawa et al., 1992; Bandettini et al., 1992). Specific cortical systems, typically somatosensory, motor, vision, or memory-related, are currently being studied with varied experimental paradigms. The basis of the fMRI signal is the blood oxygenation level-dependent (BOLD) response which arises when cerebral autoregulation causes an increase in local blood flow in response to neural activity (Ogawa et al., 1990). This increase in local flow is actually a physiologic overcompensation, so that the relative percentage of deoxygenated hemoglobin actually decreases in the local venous vasculature (Fox et al., 1988). Deoxygenated hemoglobin acts as a paramagnetic agent while oxyhemoglobin is diamagnetic, and different ratios of these species affect the spins of neighboring water protons (Pauling and Coryell, 1936). The relative spin effect is detected by the MR imager, and the image is reconstructed by twodimensional Fourier analysis. fMRI methods are optimized to acquire multiple images of the relevant volume very quickly, resulting in a four-dimensional $(x, y, z$, and $t)$ data set with spatial (2-4 $\mathrm{mm})$ and temporal resolution (1-2 s) acceptable for studying the visual cortex.

The output data in its raw form is an 8-bit grayscale intensity value for each voxel and time point. The BOLD response causes the intensity values of individual voxels in any given data set to be representative of the relative blood oxygenation level of the tissue encoded by those voxels. These intensity values are compared with values acquired from a baseline or resting condition to determine the ratio, or percentage, of signal change. Most fMRI methods utilize a type of correlative design, which can be applied to either blocks of sustained stimulation, or to rapidly presented stimuli with overlapping BOLD responses. However, a notable exception to subtraction is the phase-encoded design, which instead uses Fourier analysis and is discussed in much greater detail in subsequent chapters. 


\subsubsection{Retinotopic Mapping}

Much of the fMRI literature falls in the domain of visual neuroscience. Consequently, we have learned quite a lot about the organization of human visual cortex in the last few years. One of the most fundamental findings has been the demonstration and visualization of the retinotopic organization of multiple visual areas (Fig. 1-4), including V1 and extrastriate areas V2, V3, V3A, VP, V4v, and MT (Sereno et al., 1995; DeYoe et al., 1996; Engel et al., 1997). These visual areas are comprised of multiple representations of retinotopic visual space, and are thought to be responsible for progressively more sophisticated analyses of visual stimuli, operating both serially and in parallel (Maunsell and Van Essen, 1983b; Felleman and Van Essen, 1991). In general, ventral visual areas are more sensitive to object features, while dorsal visual areas are more sensitive to visual motion and space. For example, V4v contains neurons responsive to complex stimulus features like contours and color (Zeki, 1973; Gallant et al., 1996), while V3, V3A and MT contain neurons selective for direction and speed of motion (Albright, 1984; Tootell et al., 1997; Gegenfurtner et al., 1997). For this reason, ventral visual areas are often referred to as belonging to a 'what' pathway of visual processing, while dorsal visual areas are often referred to as forming a 'where' pathway (Ungerleider and Haxby, 1994). It is easiest to study retinotopic organization using techniques for visualizing fMRI results on flattened computer models of individual subjects' cortical surfaces (Fischl et al., 1999; Dale et al., 1999). The retinotopic organization in humans seems to agree with maps derived in monkeys from traditional electrophysiological or fMRI recordings (Felleman and Van Essen, 1991; Van Essen et al., 2001). 


\subsection{Functional Imaging of Amblyopia}

In recent years, functional neuroimaging has allowed the correlation of physiologic data with psychophysics in human observers. With the advent of positron-emission tomography (PET), magnetoencephalography (MEG), and functional magnetic resonance imaging (fMRI), several investigators have examined cortical function in humans with amblyopia and other visual disorders. For example, in addition to amblyopia, functional neuroimaging has also been used to study congenital achromatopsia, albinism, and lesions to the optic radiations (Morland et al., 2001; Baseler et al., 2002). However, many more studies have been devoted to amblyopia than to any other visual disorder.

\subsubsection{PET Studies}

All of the PET studies of amblyopia have reported decreased signal in visual cortex with amblyopic eye stimulation compared to fellow eye stimulation, regardless of amblyopic subtype or whether a metabolic $\left(\left[{ }^{18} \mathrm{~F}\right]\right.$-2-deoxyglucose $)$ or perfusion $\left(\mathrm{H}_{2}{ }^{15} \mathrm{O}\right)$ tracer was used. This suggests that the metabolic rate in visual cortex following amblyopic eye stimulation is relatively lower than for the fellow eye. In addition, these were the first imaging studies to actually find an inverse linear relationship between the amblyopic eye acuity deficit and the monocular calcarine regional blood flow change (Demer et al., 1997). These findings are consistent with metabolic findings showing shifts in the extent of the metabolic marker $\mathrm{CO}$ in amblyopic macaque striate cortex (Horton et al., 1997). However, additional reported results are less consistent across studies. Some studies reported less activation of primary visual cortex (V1) by the amblyopic than the fellow eye (Demer et al., 1988; Kabasakal et al., 1995). Others reported similar activation of Brodmann's area (BA) 17 (V1) after stimulation through either eye (Imamura et al., 1997), with activation differences localized solely to BAs 18 and 19. Still others reported broad signal decreases throughout visual cortex (Choi et al., 2002; Mizoguchi et al., 2005). Different stimuli and viewing conditions further confound comparisons between these studies.

Methodologically, it is important to note that the temporal resolution of PET is quite limited. Acquisitions are taken with the subject viewing a given stimulus condition for an extended period of time, e.g., for several minutes. This length of time represents a long period over which neural activity, assessed as glucose uptake or rCBF changes, is pooled. Furthermore, 
the spatial resolution is merely adequate compared with $\mathrm{fMRI}$, typically on the order of $1 \mathrm{~cm}^{3}$ in PET studies. Finally, PET applications can be limited in certain populations, because they require intravenous injection of a radioactive tracer compound. Tracer doses must be carefully titrated, limiting the amount of experimental exposure. Furthermore, the availability of radioactive tracer can also be a problem at sites without a cyclotron and radiochemist. Therefore, PET is usually of limited use in serial applications, as might be ideal for studying normal and abnormal developmental processes.

\subsubsection{MEG Studies}

MEG experiments provide complementary information to PET, with much better temporal resolution (at the msec level), but greater uncertainty for signal localization (Ueno and Iramina, 1990; Mosher et al., 1993; Tomita et al., 1996). The only reported MEG study of amblyopia described evoked responses to 1-2 cpd gratings in strabismics. Amblyopic eye stimulation resulted in signals that were lower in amplitude and delayed relative to fellow eye stimulation. The amount of the delay varied between subjects, but the fellow eye response typically peaked at $90 \%$ of the amblyopic eye latency. The maximal evoked responses were shifted to lower spatial frequencies in the amblyopic eye than in the normal eye, consistent with known psychophysical deficits. These signals were estimated to arise from the area of the V1/V2 border (Anderson et al., 1999).

\subsection{3 fMRI Studies}

The superior spatial resolution of fMRI has recently been applied to the study of amblyopia. Briefly, this has allowed quantification of reduced activation extent within anatomically specific regions of visual cortex (Goodyear et al., 2000; Algaze et al., 2002; Goodyear et al., 2002), while also confirming with more anatomical definition the decreased signal magnitudes seen with PET (Barnes et al., 2001; Choi et al., 2001; Lee et al., 2001; Liu et al., 2004). Furthermore, a few of these studies have also compared anisometropic and strabismic amblyopia, finding less activation for anisometropes at high spatial frequencies (Choi et al., 2001), but more impaired responses to binocular stimuli from strabismics (Lee et al., 2001). A more detailed discussion of these results follows. 
The first fMRI study with amblyopic subjects utilized four subjects with strabismic amblyopia and six control subjects (Goodyear et al., 2000). The subjects were presented gratings at a fixed suprathreshold contrast (22\%). Six spatial frequencies were chosen $(0.5,1,2,4,8$, and $12 \mathrm{cpd}$ ). The fMRI experiment was comprised of individual trials using an event-related design, where the six spatial frequencies were presented in random order three times each for 3 seconds each trial with an interstimulus interval of 20 seconds. To measure the fMRI response, this study used a region-of-interest (ROI) analysis defined as the intersection of an anatomically-defined area including all of V1 and some of V2 and a functional mask composed from the union of all voxels with significant fMRI responses from any stimulus.

The results of the Goodyear et al. (2000) experiment showed that the magnitude of the fMRI response (\% signal change) covaried with the perceived contrast of each grating in both normal and amblyopic subjects, regardless of spatial frequency. Only one of the four amblyopic subjects showed a large depression in the high spatial frequency portion of the contrast perception curve. Interestingly, this one subject also showed analogously lower MR signal for those stimuli, so the signal magnitude was still predicted by the reduced perceived contrast. For all subjects with amblyopia, the total number of activated voxels meeting the significance criterion decreased for amblyopic relative to fellow eye stimulation for all spatial frequencies. These findings suggest that the extent of cortical activity is less from amblyopic eye stimulation even when the percept is equivalent to that obtained with fellow eye stimulation.

Unfortunately, the conservative ROI used in this study made it impossible to know the full extent of the functional deficit in other visual (or nonvisual) areas. Furthermore, extrastriate areas with less robust activation than V1 may require more trials than were used in this study to achieve statistical significance. This study was also hindered by a small number of participants, and the fact that only one of the four amblyopic subjects showed a significantly depressed contrast perception function in the amblyopic eye.

In this study, Goodyear et al. (2000) reported dissociation in strabismic amblyopes between the fMRI signal magnitude and total activated voxels. There are several possibilities for the meaning of the decreased MR signal extent. Since the deficit seen here was primarily one of extent and not signal strength, the simplest explanation might be that fewer cortical neurons were responding to a given stimulus. However, even this effect could arise from numerous sources. For example, less fMRI activity may reflect an actual reduction in number of cortical neurons 
that can be stimulated by the amblyopic eye, fewer connections between input and output layers of V1, or even feedback effects from other visual areas which actively inhibit V1 neurons from responding. Furthermore, there are also methodological considerations that could have resulted in reduced areas of activation, like greater motion for amblyopic eye viewing. Finally, fMRI may not always be able to achieve the spatial resolution necessary to disentangle extent and magnitude of BOLD signal, since functional imaging of even the relatively large ocular dominance columns is difficult in normally sighted subjects using standard techniques and available MR imagers (Menon et al., 1997; Goodyear and Menon, 2001; Cheng et al., 2001).

The other landmark fMRI study of amblyopia presented both suprathreshold and subthreshold grating stimuli to the amblyopic eyes of ten strabismics, but surprisingly did not use control subjects (Barnes et al., 2001). All stimuli were of a constant high contrast level (50\%) but were chosen such that one grating stimulus was within the "visible" acuity range (4 cpd) while the other was "invisible," above the acuity limit (11-22 cpd, depending on the severity of the amblyopic deficit). This was the first imaging study to attempt retinotopic mapping of multiple visual areas in amblyopic subjects. Retinotopic mapping was conducted in the fellow eye to derive visual area boundaries, but was not attempted through the amblyopic eye.

The "visible" stimulus produced less activation in all subjects in all visual areas when viewed through the amblyopic eye compared to the fellow eye, possibly correlated with lower perceived contrast of these stimuli through the amblyopic eye, as in Goodyear et al. (2000). Interestingly, the decreased activation was not related to the acuity deficit in the amblyopic eye, a finding somewhat in conflict with the earlier PET data (Demer et al., 1997).

This study also demonstrated the novel finding of cortical activity in response to the "invisible" amblyopic stimuli in two subjects, localized to the dorsal V3/V3A border. This result deserves mention because it implies residual cortical visual processing in amblyopia, even if the visual activity is insufficient to reach conscious perception. Interestingly, recent reports of cortical activity in 'blindsight' patients with V1 lesions also show residual processing in dorsal extrastriate cortex, including V3 and V3A (Stoerig et al., 1998; Bittar et al., 1999).

The first fMRI study to directly address the differences between strabismic and anisometropic amblyopia used stimuli over a range of spatial and temporal frequencies (0.25 to 2 cpd and 2 to $16 \mathrm{~Hz}$ ) (Choi et al., 2001). Stimuli were high contrast reversing checkerboards, varying parametrically in one of the two variable dimensions. Once again, this group found 
reduced calcarine activation with amblyopic eye compared with fellow eye stimulation, but no attempt was made to localize the activation to individual visual areas outside of V1. Strabismic amblyopes differed from anisometropic amblyopes in the spatial frequency dependence of the reduced activity, with the strabismic range extending down to $0.25 \mathrm{cpd}$ while the anisometropic range extended only to $1 \mathrm{cpd}$. Anisometropic subjects also exhibited significantly less fMRI response through the amblyopic eye from 4 to $8 \mathrm{~Hz}$, while the strabismic group showed no difference. Unfortunately, this group did not include any psychophysical measures (e.g., a contrast sensitivity function measurement) in their design for intrasubject comparison with the fMRI data, so the relationship of the fMRI data to the subjects' percept is not known.

Finally, Lee et al. (2001) also compared strabismic and anisometropic amblyopia using fMRI, but again without a control population (Lee et al., 2001). This group stimulated the amblyopic and fellow eyes independently and calculated an index of binocularly responding voxels within an anatomical region of interest including V1 and some extrastriate cortex. The index was defined as the intersection of voxels responding to stimulation from both eyes divided by the union of voxels stimulated by either eye. The results showed that strabismic amblyopes have significantly fewer binocular cortical voxels $(17 \pm 3 \%$ vs. $35 \pm 5 \%$ for anisometropic amblyopes).

It is remarkable that this effect could be shown using fMRI, since the spatial resolution of the technique is not as fine as the size of the ocular dominance columns (ODCs) in V1 (i.e., 'typical' fMRI has a resolution of 3-4 $\mathrm{mm}$, but the ODCs are only $0.5 \mathrm{~mm}$ wide). However, the results from this novel technique agree well with available electrophysiology, suggesting that binocularity is more impaired in strabismic amblyopia (Kiorpes et al., 1998). In contrast, anisometropic amblyopia seems to be better characterized by loss of high spatial frequency channels (Movshon et al., 1987). Indeed, the anisometropic amblyopes showed less MR signal at high spatial frequencies, while the strabismics did not. This apparent double dissociation raises interesting possibilities for future amblyopia studies.

In summary, the previous neuroimaging literature has informed us that these techniques can be used to demonstrate reduced signal strength, extent of activation, and cortical ocular dominance and binocularity. However, none have specifically contrasted activity in the central and peripheral visual fields, an interesting omission since the central field is known to be particularly impaired in amblyopia while the peripheral field can be normal. This project aims to 
investigate the cortical substrates of amblyopia in adults and children, with a specific interest in the organization of the central visual field. 


\subsection{Figures}

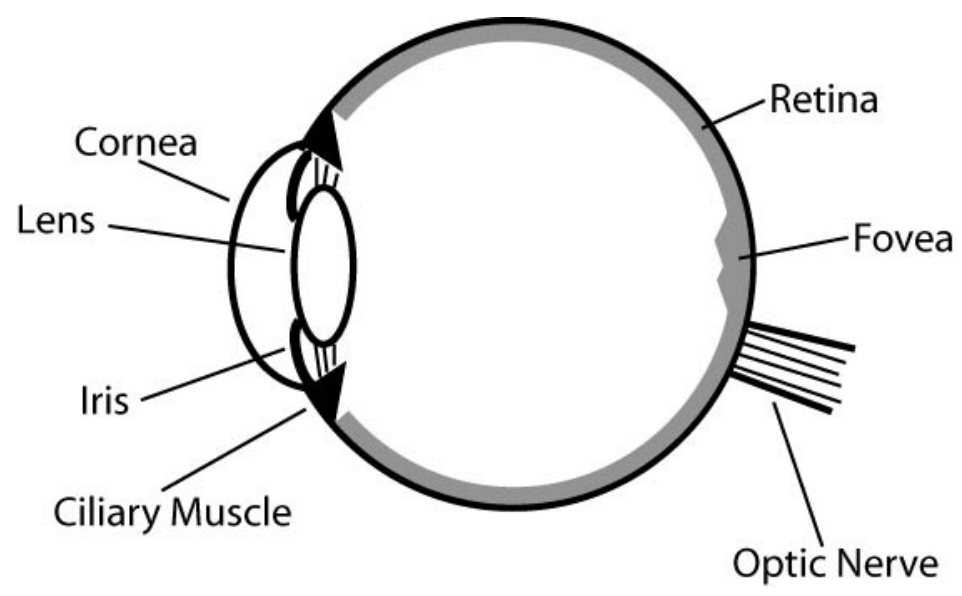

FIGURE 1-1. CROSS-SECTION OF A HUMAN EYE. Light enters and is refracted through the cornea, and is then focused onto the retina by the lens. Cones are most concentrated in the fovea, where visual acuity is also at the highest level. 


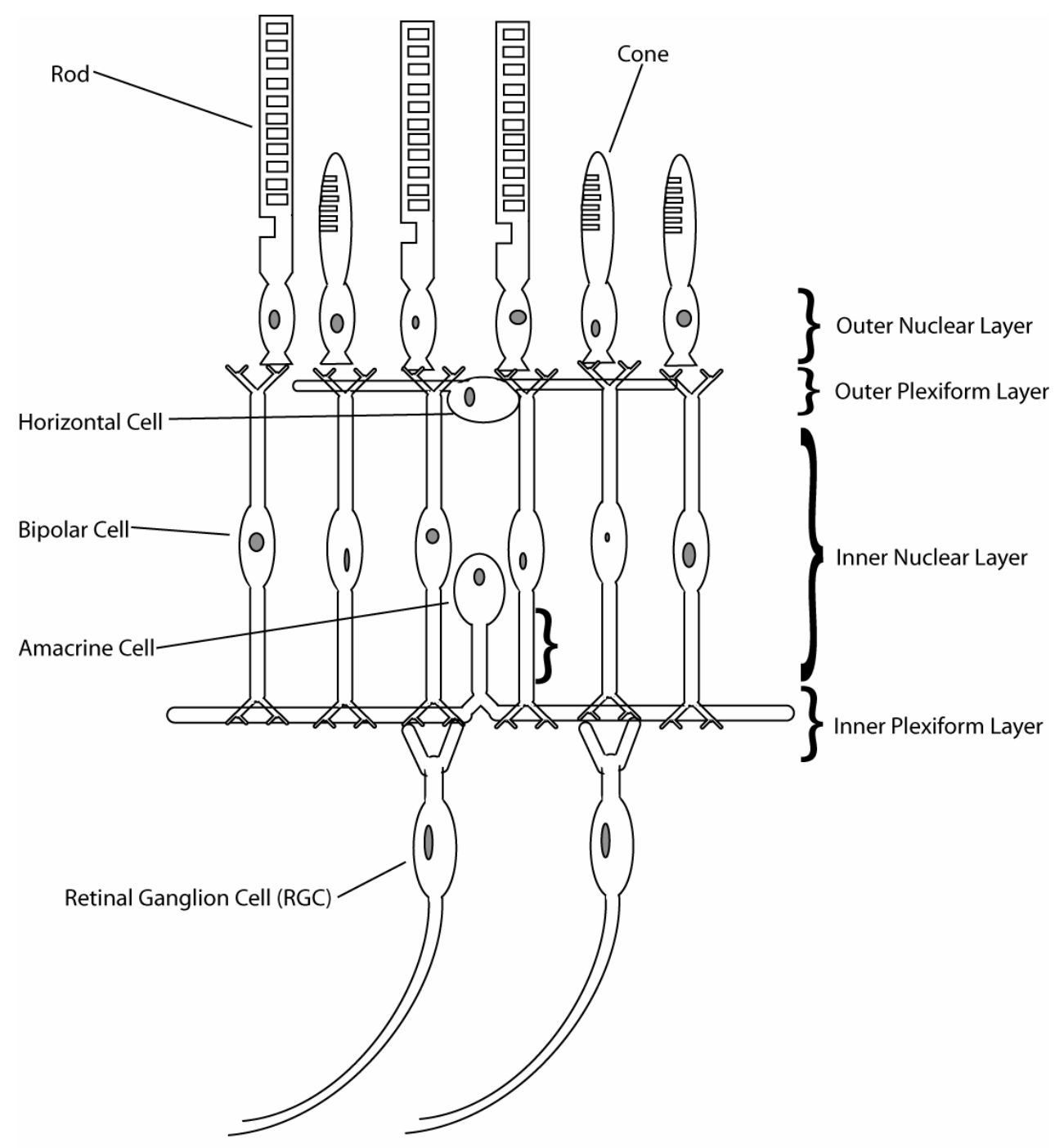

RGC Axons Exit the Retina as the Optic Nerve

Figure 1-2. Neuronal TyPeS In THE Human Retina. Light is transduced by the photoreceptors (i.e., the rods and cones), whose cell bodies are located in the outer nuclear layer. The encoded visual information is synaptically transmitted in the outer plexiform layer from the photoreceptors bipolar cells, which in turn synapse on retinal ganglion cells in the inner plexiform layer. Horizontal and amacrine cells make lateral connections in the outer and inner plexiform layers. Ganglion cell axons exiting the retina form the optic nerve. 


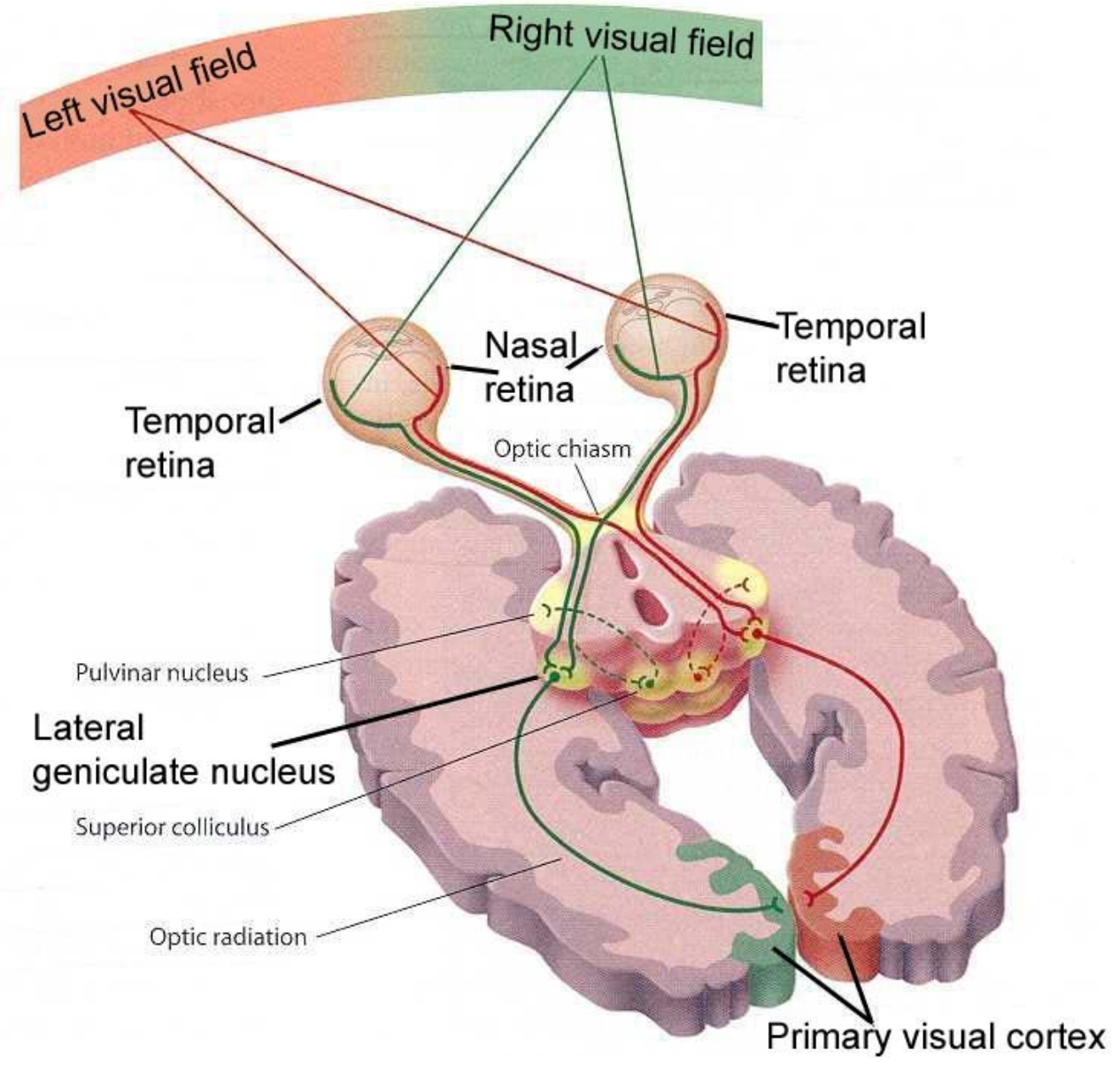

Figure 1-3. The Central Visual Pathways. Information about visual space is transmitted through the contralateral lateral geniculate nucleus to primary visual cortex (V1), where the amblyopic deficit is thought to occur. V1 is the first stage of the visual system where information from the eyes is integrated, since the LGN is functionally monocular. This illustration is adapted from another source (Goldstein, 1999). 


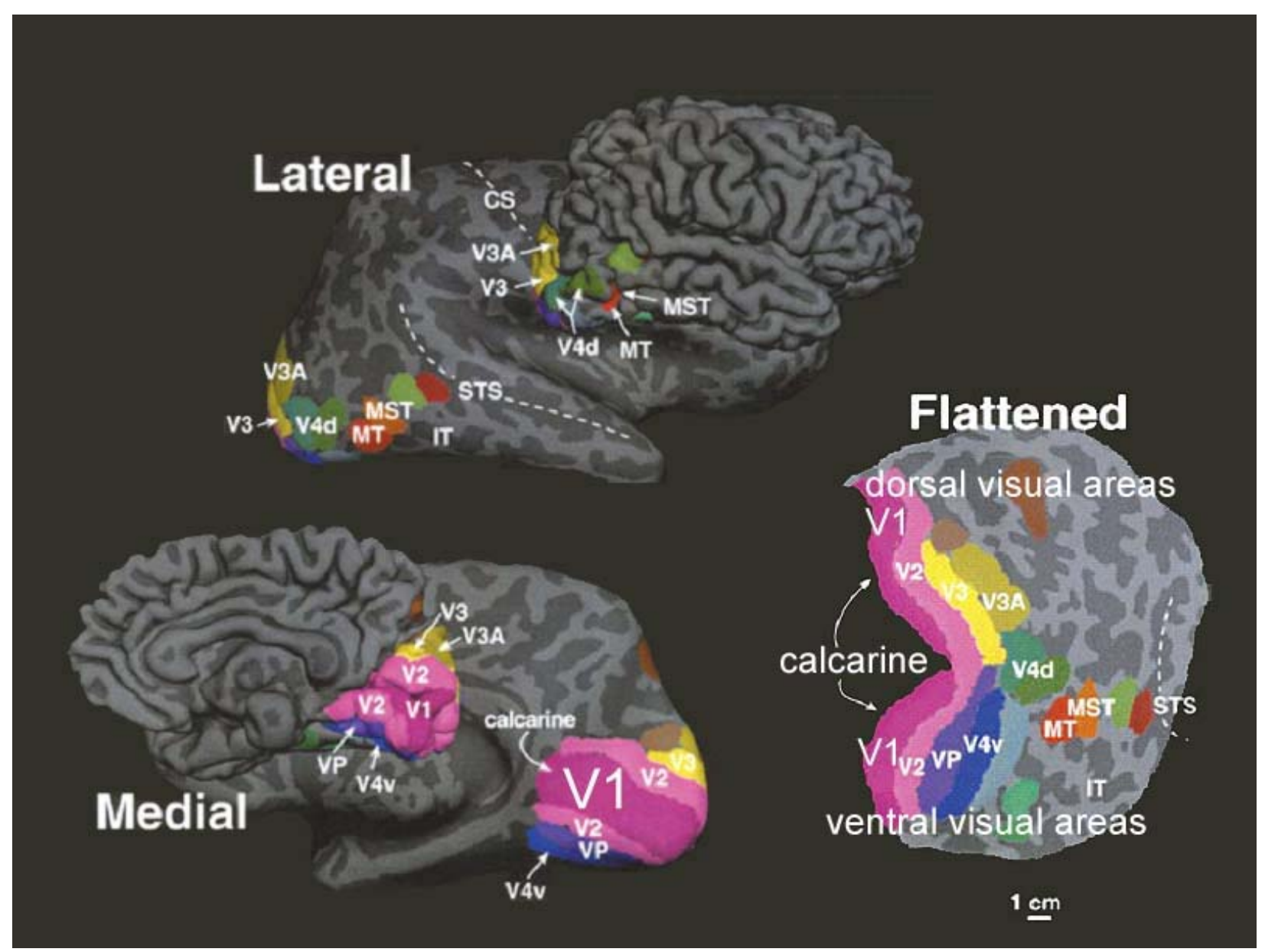

Figure 1-4. Multiple Retinotopic Areas in Visual Cortex. V1 is found in the calcarine sulcus, and is artificially split at the representation of the horizontal meridian in the flattened patch. Higher visual areas are organized ventrally (V2v, VP, V4v) and dorsally (V2d, V3, V3A) from V1. Motion-sensitive area MT is located laterally, in the posterior temporal lobe. This illustration is adapted from another source (Bear et al., 2001). 

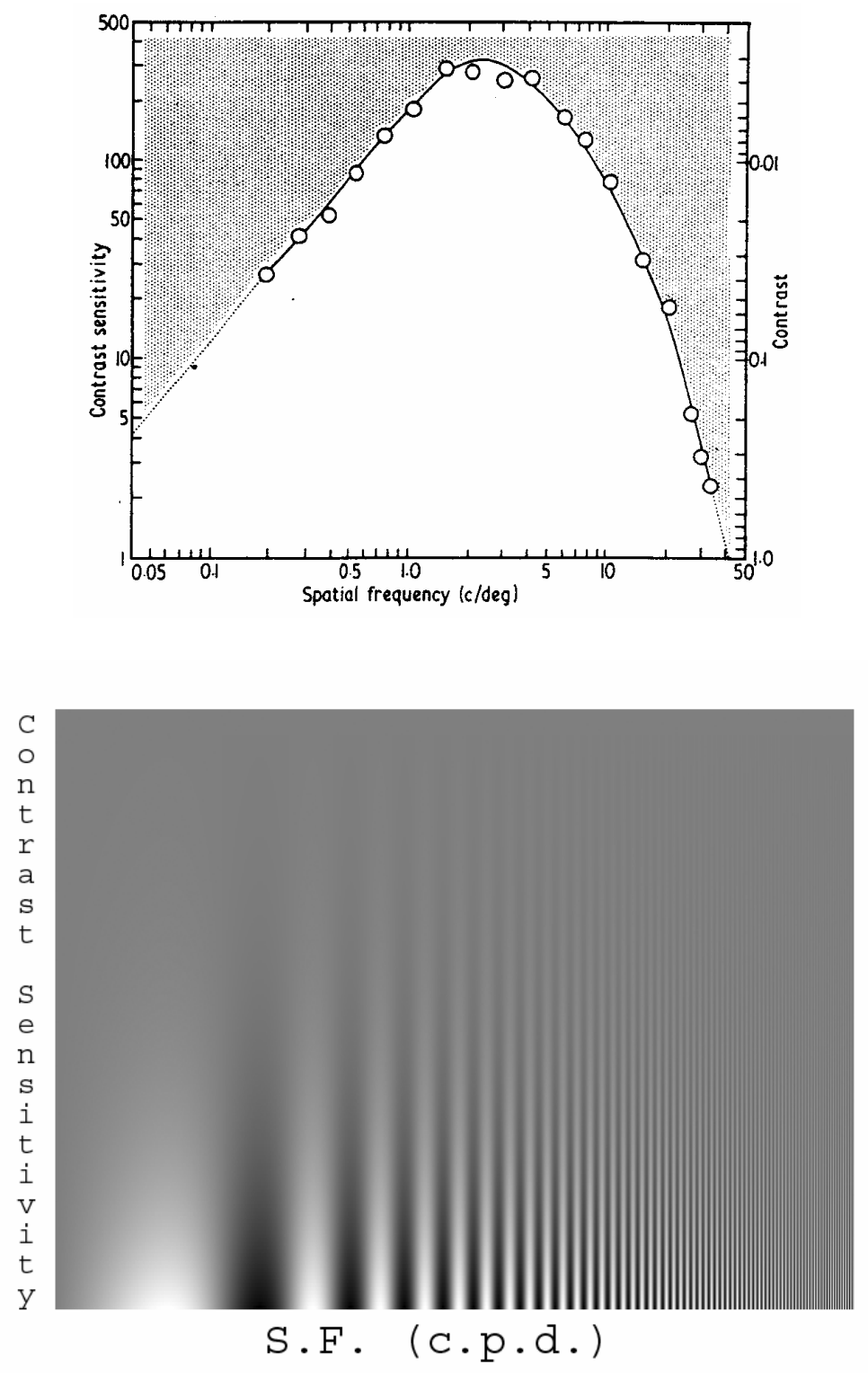

Figure 1-5. The Contrast Sensitivity Function (CSF) Has an Inverted U-Shape. Top. Human vision can detect stimuli with characteristics described under the curve. Bottom. This phenomenon is illustrated for the reader. This illustration is adapted from another source (Campbell, 1983). 


\subsection{References}

Abrahamsson M, Sjostrand J (1988) Contrast sensitivity and acuity relationship in strabismic and anisometropic amblyopia. Br J Ophthalmol 72:44-49.

Adams RJ, Courage ML (2002) Using a single test to measure human contrast sensitivity from early childhood to maturity. Vision Res 42:1205-1210.

Albright TD (1984) Direction and orientation selectivity of neurons in visual area MT of the macaque. J Neurophysiol 52:1106-1130.

Algaze A, Roberts C, Leguire L, Schmalbrock P, Rogers G (2002) Functional magnetic resonance imaging as a tool for investigating amblyopia in the human visual cortex: a pilot study. J AAPOS 6:300-308.

Allison T, McCarthy G, Nobre A, Puce A, Belger A (1994) Human extrastriate visual cortex and the perception of faces, words, numbers, and colors. Cereb Cortex 4:544-554.

Allison T, Puce A, McCarthy G (2000) Social perception from visual cues: role of the STS region. Trends Cogn Sci 4:267-278.

Anderson SJ, Holliday IE, Harding GF (1999) Assessment of cortical dysfunction in human strabismic amblyopia using magnetoencephalography (MEG). Vision Res 39:1723-1738.

Atkinson J, Braddick O, Moar K (1977) Development of contrast sensitivity over the first 3 months of life in the human infant. Vision Res 17:1037-1044.

Atkinson J (2002) The developing visual brain. Oxford: Oxford University Press.

Bandettini PA, Wong EC, Hinks RS, Tikofsky RS, Hyde JS (1992) Time course EPI of human brain function during task activation. Magn Reson Med 25:390-397.

Banks MS, Bennett PJ (1988) Optical and photoreceptor immaturities limit the spatial and chromatic vision of human neonates. J Opt Soc Am A 5:2059-2079.

Barishak YR (1992) Embryology of the eye and its adnexae. Dev Ophthalmol 24:1-142.

Barlow HB (1953) Summation and inhibition in the frog's retina. J Physiol 119:69-88.

Barnes GR, Hess RF, Dumoulin SO, Achtman RL, Pike GB (2001) The cortical deficit in humans with strabismic amblyopia. J Physiol 533:281-297.

Barrett BT, Bradley A, McGraw PV (2004) Understanding the neural basis of amblyopia. Neuroscientist 10:106-117. 
Baseler HA, Brewer AA, Sharpe LT, Morland AB, Jagle H, Wandell BA (2002) Reorganization of human cortical maps caused by inherited photoreceptor abnormalities. Nat Neurosci $5: 364-370$.

Bear MF, Connors BW, Paradiso MA (2001) Neuroscience exploring the brain. Baltimore, Md: Lippincott Williams \& Wilkins.

Bittar RG, Ptito M, Faubert J, Dumoulin SO, Ptito A (1999) Activation of the remaining hemisphere following stimulation of the blind hemifield in hemispherectomized subjects. Neuroimage 10:339-346.

Blakemore C (1976) Modification of visual function by early visual experience. Bull Schweiz Akad Med Wiss 32:13-28.

Bodis-Wollner I (1980) Detection of visual defects using the contrast sensitivity function. Int Ophthalmol Clin 20:135-153.

Boothe RG, Kiorpes L, Hendrickson A (1982) Anisometropic amblyopia in Macaca nemestrina monkeys produced by atropinization of one eye during development. Invest Ophthalmol Vis Sci 22:228-233.

Bosking WH, Zhang Y, Schofield B, Fitzpatrick D (1997) Orientation selectivity and the arrangement of horizontal connections in tree shrew striate cortex. J Neurosci 17:2112-2127.

Boycott BB, Wassle H (1974) The morphological types of ganglion cells of the domestic cat's retina. J Physiol 240:397-419.

Braddick OJ, Wattam-Bell J, Atkinson J (1986) Orientation-specific cortical responses develop in early infancy. Nature 320:617-619.

Bradley A, Freeman RD (1982) Contrast sensitivity in children. Vision Res 22:953-959.

Bradley A, Freeman RD (1981) Contrast sensitivity in anisometropic amblyopia. Invest Ophthalmol Vis Sci 21:467-476.

Burian HM (1967) The behavior of the amblyopic eye under reduced illumination and the theory of functional amblyopia. Doc Ophthalmol 23:189-202.

Campbell FW (1983) Why do we measure contrast sensitivity? Behav Brain Res 10:87-97.

Campbell FW, Robson JG (1968) Application of Fourier analysis to the visibility of gratings. J Physiol 197:551-566.

Campos E (1995) Amblyopia. Surv Ophthalmol 40:23-39.

Candy TR (2000) Neuronal correlates of amblyopia in the visual cortex of macaque monkeys with experimental strabismus and anisometropia, by L. Kiorpes, D. Kiper, L. O'Keefe, J. Cavanaugh, and A. Movshon. J neuroscience 18:6411-24, 1998. Surv Ophthalmol 45:166. 
Chalupa LM, Lia B (1991) The nasotemporal division of retinal ganglion cells with crossed and uncrossed projections in the fetal rhesus monkey. J Neurosci 11:191-202.

Cheng K, Waggoner RA, Tanaka K (2001) Human ocular dominance columns as revealed by high-field functional magnetic resonance imaging. Neuron 32:359-374.

Choi MY, Lee DS, Hwang JM, Choi DG, Lee KM, Park KH, Yu YS (2002) Characteristics of glucose metabolism in the visual cortex of amblyopes using positron-emission tomography and statistical parametric mapping. J Pediatr Ophthalmol Strabismus 39:11-19.

Choi MY, Lee KM, Hwang JM, Choi DG, Lee DS, Park KH, Yu YS (2001) Comparison between anisometropic and strabismic amblyopia using functional magnetic resonance imaging. Br J Ophthalmol 85:1052-1056.

Chua B, Mitchell P (2004) Consequences of amblyopia on education, occupation, and long term vision loss. Br J Ophthalmol 88:1119-1121.

Cobb SR, MacDonald CF (1978) Resolution acuity in astigmats: evidence for a critical period in the human visual system. Br J Physiol Opt 32:38-49.

Conley M, Fitzpatrick D (1989) Morphology of retinogeniculate axons in the macaque. Vis Neurosci 2:287-296.

Crawford ML, Harwerth RS (2004) Ocular dominance column width and contrast sensitivity in monkeys reared with strabismus or anisometropia. Invest Ophthalmol Vis Sci 45:3036-3042.

Crewther DP, Crewther SG (1990) Neural site of strabismic amblyopia in cats: spatial frequency deficit in primary cortical neurons. Exp Brain Res 79:615-622.

Culham JC, Dukelow SP, Vilis T, Hassard FA, Gati JS, Menon RS, Goodale MA (1999) Recovery of fMRI activation in motion area MT following storage of the motion aftereffect. J Neurophysiol 81:388-393.

Curcio CA, Sloan KR, Jr., Packer O, Hendrickson AE, Kalina RE (1987) Distribution of cones in human and monkey retina: individual variability and radial asymmetry. Science 236:579-582.

Cynader MS (1982) Competitive neuronal interactions underlying amblyopia. Hum Neurobiol $1: 35-39$.

Dacey DM (1994) Physiology, morphology and spatial densities of identified ganglion cell types in primate retina. Ciba Found Symp 184:12-28.

Dacey DM (2000) Parallel pathways for spectral coding in primate retina. Annu Rev Neurosci 23:743-775.

Dale AM, Fischl B, Sereno MI (1999) Cortical surface-based analysis. I. Segmentation and surface reconstruction. Neuroimage 9:179-194. 
Daw NW (1998) Critical periods and amblyopia. Arch Ophthalmol 116:502-505.

Daw N (1995) Visual development. New York: Plenum Press.

DeAngelis GC, Freeman RD, Ohzawa I (1994) Length and width tuning of neurons in the cat's primary visual cortex. J Neurophysiol 71:347-374.

Delint PJ, Weissenbruch C, Berendschot TT, Norren DV (1998) Photoreceptor function in unilateral amblyopia. Vision Res 38:613-617.

Demer JL, Grafton S, Marg E, Mazziotta JC, Nuwer M (1997) Positron-emission tomographic study of human amblyopia with use of defined visual stimuli. J AAPOS 1:158-171.

Demer JL, von Noorden GK, Volkow ND, Gould KL (1988) Imaging of cerebral blood flow and metabolism in amblyopia by positron emission tomography. Am J Ophthalmol 105:337-347.

Desimone R, Schein SJ, Moran J, Ungerleider LG (1985) Contour, color and shape analysis beyond the striate cortex. Vision Res 25:441-452.

Dews PB, Wiesel TN (1970) Consequences of monocular deprivation on visual behaviour in kittens. J Physiol 206:437-455.

DeYoe EA, Carman GJ, Bandettini P, Glickman S, Wieser J, Cox R, Miller D, Neitz J (1996) Mapping striate and extrastriate visual areas in human cerebral cortex. Proc Natl Acad Sci U S A 93:2382-2386.

Dobson V, Teller DY (1978) Visual acuity in human infants: a review and comparison of behavioral and electrophysiological studies. Vision Res 18:1469-1483.

Dobson V, Teller DY, Lee CP, Wade B (1978) A behavioral method for efficient screening of visual acuity in young infants. I. Preliminary laboratory development. Invest Ophthalmol Vis Sci 17:1142-1150.

Dowling JE, Boycott BB (1966) Organization of the primate retina: electron microscopy. Proc R Soc Lond B Biol Sci 166:80-111.

Ellemberg D, Lewis TL, Liu CH, Maurer D (1999) Development of spatial and temporal vision during childhood. Vision Res 39:2325-2333.

Engel SA, Glover GH, Wandell BA (1997) Retinotopic organization in human visual cortex and the spatial precision of functional MRI. Cereb Cortex 7:181-192.

Erwin E, Baker FH, Busen WF, Malpeli JG (1999) Relationship between laminar topology and retinotopy in the rhesus lateral geniculate nucleus: results from a functional atlas. J Comp Neurol 407:92-102. 
Eysel UT, Shevelev IA, Lazareva NA, Sharaev GA (1998) Orientation tuning and receptive field structure in cat striate neurons during local blockade of intracortical inhibition. Neuroscience 84:25-36.

Fantz RL (1965) Visual perception from birth as shown by pattern selectivity. Ann N Y Acad Sci 118:793-814.

Felleman DJ, Van Essen DC (1991) Distributed hierarchical processing in primate cerebral cortex. Cereb Cortex 1:1-47.

Fenstemaker SB, Kiorpes L, Movshon JA (2001) Effects of experimental strabismus on the architecture of macaque monkey striate cortex. J Comp Neurol 438:300-317.

Fischl B, Sereno MI, Dale AM (1999) Cortical surface-based analysis. II: Inflation, flattening, and a surface-based coordinate system. Neuroimage 9:195-207.

Flom MC, Bedell HE (1985) Identifying amblyopia using associated conditions, acuity, and nonacuity features. Am J Optom Physiol Opt 62:153-160.

Flom MC, Neumaier RW (1966) Prevalence of amblyopia. Public Health Rep 81:329-341.

Florence SL, Casagrande VA (1987) Organization of individual afferent axons in layer IV of striate cortex in a primate. J Neurosci 7:3850-3868.

Fox PT, Raichle ME, Mintun MA, Dence C (1988) Nonoxidative glucose consumption during focal physiologic neural activity. Science 241:462-464.

Gallant JL, Connor CE, Rakshit S, Lewis JW, Van Essen DC (1996) Neural responses to polar, hyperbolic, and Cartesian gratings in area V4 of the macaque monkey. J Neurophysiol 76:2718-2739.

Gegenfurtner KR, Kiper DC, Levitt JB (1997) Functional properties of neurons in macaque area V3. J Neurophysiol 77:1906-1923.

Gibson JJ (1950) The perception of visual surfaces. Am J Psychol 63:367-384.

Gibson JJ, Flock H (1962) The apparent distance of mountains. Am J Psychol 75:501-503.

Gilbert CD (1998) Adult cortical dynamics. Physiol Rev 78:467-485.

Gillespie DC, Lampl I, Anderson JS, Ferster D (2001) Dynamics of the orientation-tuned membrane potential response in cat primary visual cortex. Nat Neurosci 4:1014-1019.

Goldstein EB (1999) Sensation and perception. Pacific Grove: Brooks/Cole Pub.

Goodyear BG, Menon RS (2001) Brief visual stimulation allows mapping of ocular dominance in visual cortex using fMRI. Hum Brain Mapp 14:210-217. 
Goodyear BG, Nicolle DA, Humphrey GK, Menon RS (2000) BOLD fMRI response of early visual areas to perceived contrast in human amblyopia. J Neurophysiol 84:1907-1913.

Goodyear BG, Nicolle DA, Menon RS (2002) High resolution fMRI of ocular dominance columns within the visual cortex of human amblyopes. Strabismus 10:129-136.

Grigg J, Thomas R, Billson F (1996) Neuronal basis of amblyopia: a review. Indian J Ophthalmol 44:69-76.

Harrad R (1996) Psychophysics of suppression. Eye 10 ( Pt 2):270-273.

Hartline HK (1938) Response of single optic nerve fibers of the vertebrate eye to illumination of the retina. Am J Physiol 121:400-415.

Harwerth RS, Smith EL, III, Crawford ML, von Noorden GK (1990) Behavioral studies of the sensitive periods of development of visual functions in monkeys. Behav Brain Res 41:179198.

Harwerth RS, Smith EL, III, Duncan GC, Crawford ML, von Noorden GK (1986) Multiple sensitive periods in the development of the primate visual system. Science 232:235-238.

Hess RF (2001) Amblyopia: sight unseen. Clin Exp Optom 84:321-336.

Hess RF, France TD, Tulunay-Keesey U (1981) Residual vision in humans who have been monocularly deprived of pattern stimulation in early life. Exp Brain Res 44:295-311.

Hess RF, Howell ER (1977) The threshold contrast sensitivity function in strabismic amblyopia: evidence for a two type classification. Vision Res 17:1049-1055.

Hess RF, Jacobs RJ (1979) A preliminary report of acuity and contour interactions across the amblyope's visual field. Vision Res 19:1403-1408.

Hess RF, Pointer JS (1985) Differences in the neural basis of human amblyopia: the distribution of the anomaly across the visual field. Vision Res 25:1577-1594.

Hevner RF (2000) Development of connections in the human visual system during fetal midgestation: a DiI-tracing study. J Neuropathol Exp Neurol 59:385-392.

Hicks TP, Lee BB, Vidyasagar TR (1983) The responses of cells in macaque lateral geniculate nucleus to sinusoidal gratings. J Physiol 337:183-200.

Hillis A, Flynn JT, Hawkins BS (1983) The evolving concept of amblyopia: a challenge to epidemiologists. Am J Epidemiol 118:192-205.

Hirsch JA, Gilbert CD (1991) Synaptic physiology of horizontal connections in the cat's visual cortex. J Neurosci 11:1800-1809.

Horton JC (1984) Cytochrome oxidase patches: a new cytoarchitectonic feature of monkey visual cortex. Philos Trans R Soc Lond B Biol Sci 304:199-253. 
Horton JC, Dagi LR, McCrane EP, de Monasterio FM (1990) Arrangement of ocular dominance columns in human visual cortex. Arch Ophthalmol 108:1025-1031.

Horton JC, Hedley-Whyte ET (1984) Mapping of cytochrome oxidase patches and ocular dominance columns in human visual cortex. Philos Trans R Soc Lond B Biol Sci 304:255272.

Horton JC, Hocking DR (1996) Pattern of ocular dominance columns in human striate cortex in strabismic amblyopia. Vis Neurosci 13:787-795.

Horton JC, Hocking DR, Kiorpes L (1997) Pattern of ocular dominance columns and cytochrome oxidase activity in a macaque monkey with naturally occurring anisometropic amblyopia. Vis Neurosci 14:681-689.

Horton JC, Stryker MP (1993) Amblyopia induced by anisometropia without shrinkage of ocular dominance columns in human striate cortex. Proc Natl Acad Sci U S A 90:5494-5498.

Hubel DH, Wiesel TN (1962) Receptive fields, binocular interaction and functional architecture in the cat's visual cortex. J Physiol 160:106-154.

Hubel DH, Wiesel TN (1965) Binocular interaction in striate cortex of kittens reared with artificial squint. J Neurophysiol 28:1041-1059.

Hubel DH, Wiesel TN (1968) Receptive fields and functional architecture of monkey striate cortex. J Physiol 195:215-243.

Hubel DH, Wiesel TN (1969) Anatomical demonstration of columns in the monkey striate cortex. Nature 221:747-750.

Hubel DH, Wiesel TN (1972) Laminar and columnar distribution of geniculo-cortical fibers in the macaque monkey. J Comp Neurol 146:421-450.

Hubel DH, Wiesel TN (1977) Ferrier lecture. Functional architecture of macaque monkey visual cortex. Proc R Soc Lond B Biol Sci 198:1-59.

Hubel DH, Wiesel TN, LeVay S (1976) Functional architecture of area 17 in normal and monocularly deprived macaque monkeys. Cold Spring Harb Symp Quant Biol 40:581-589.

Imamura K, Richter H, Fischer H, Lennerstrand G, Franzen O, Rydberg A, Andersson J, Schneider H, Onoe H, Watanabe Y, Langstrom B (1997) Reduced activity in the extrastriate visual cortex of individuals with strabismic amblyopia. Neurosci Lett 225:173-176.

Jacobs DS, Blakemore C (1988) Factors limiting the postnatal development of visual acuity in the monkey. Vision Res 28:947-958.

Kabasakal L, Devranoglu K, Arslan O, Erdil TY, Sonmezoglu K, Uslu I, Tolun H, Isitman AT, Ozker K, Onsel C (1995) Brain SPECT evaluation of the visual cortex in amblyopia. J Nucl Med 36:1170-1174. 
Kapadia MK, Ito M, Gilbert CD, Westheimer G (1995) Improvement in visual sensitivity by changes in local context: parallel studies in human observers and in V1 of alert monkeys. Neuron 15:843-856.

Kaplan E, Shapley RM (1986) The primate retina contains two types of ganglion cells, with high and low contrast sensitivity. Proc Natl Acad Sci U S A 83:2755-2757.

Kiorpes L (1992) Effect of strabismus on the development of vernier acuity and grating acuity in monkeys. Vis Neurosci 9:253-259.

Kiorpes L, Boothe RG (1981) Naturally occurring strabismus in monkeys (Macaca nemestrina). Invest Ophthalmol Vis Sci 20:257-263.

Kiorpes L, Kiper DC, O'Keefe LP, Cavanaugh JR, Movshon JA (1998) Neuronal correlates of amblyopia in the visual cortex of macaque monkeys with experimental strabismus and anisometropia. J Neurosci 18:6411-6424.

Kiorpes L, Movshon JA (1996) Amblyopia: a developmental disorder of the central visual pathways. Cold Spring Harb Symp Quant Biol 61:39-48.

Kovacs I, Polat U, Pennefather PM, Chandna A, Norcia AM (2000) A new test of contour integration deficits in patients with a history of disrupted binocular experience during visual development. Vision Res 40:1775-1783.

Kuffler SW (1953) Discharge patterns and functional organization of mammalian retina. J Neurophysiol 16:37-68.

Kwong KK, Belliveau JW, Chester DA, Goldberg IE, Weiskoff RM, Poncelet BP, Kennedy DN, Hoppel BE, Cohen MS, Turner R, Rosen B, Brady TJ (1992) Dynamic magnetic resonce imaging of human brain activity during primary sensory stimulation. Proc Natl Acad Sci U S A 89:5675-5679.

Lee KM, Lee SH, Kim NY, Kim CY, Sohn JW, Choi MY, Gyu CD, Hwang JM, Ho PK, Lee DS, Suk YY, Hyun CK (2001) Binocularity and spatial frequency dependence of calcarine activation in two types of amblyopia. Neurosci Res 40:147-153.

Leventhal AG, Rodieck RW, Dreher B (1981) Retinal ganglion cell classes in the Old World monkey: morphology and central projections. Science 213:1139-1142.

Levi DM, Harwerth RS (1980) Contrast sensitivity in amblyopia due to stimulus deprivation. $\mathrm{Br}$ J Ophthalmol 64:15-20.

Levi DM, Klein S (1982) Hyperacuity and amblyopia. Nature 298:268-270.

Levi DM, Klein SA (1985) Vernier acuity, crowding and amblyopia. Vision Res 25:979-991.

Lewis TL, Maurer D (2005) Multiple sensitive periods in human visual development: Evidence from visually deprived children. Dev Psychobiol 46:163-183. 
Liu GT, Miki A, Francis E, Quinn GE, Modestino EJ, Bonhomme GR, Haselgrove JC (2004) Eye dominance in visual cortex in amblyopia using functional magnetic resonance imaging. $J$ AAPOS 8:184-186.

Livingstone MS, Hubel DH (1982) Thalamic inputs to cytochrome oxidase-rich regions in monkey visual cortex. Proc Natl Acad Sci U S A 79:6098-6101.

Livingstone MS, Hubel DH (1984) Anatomy and physiology of a color system in the primate visual cortex. J Neurosci 4:309-356.

Livingstone MS, Hubel DH (1987) Psychophysical evidence for separate channels for the perception of form, color, movement, and depth. J Neurosci 7:3416-3468.

Lund JS (1987) Local circuit neurons of macaque monkey striate cortex: I. Neurons of laminae 4C and 5A. J Comp Neurol 257:60-92.

Lund JS, Hawken MJ, Parker AJ (1988) Local circuit neurons of macaque monkey striate cortex: II. Neurons of laminae 5B and 6. J Comp Neurol 276:1-29.

Lund JS, Wu CQ (1997) Local circuit neurons of macaque monkey striate cortex: IV. Neurons of laminae 1-3A. J Comp Neurol 384:109-126.

Lund JS, Yoshioka T (1991) Local circuit neurons of macaque monkey striate cortex: III. Neurons of laminae 4B, 4A, and 3B. J Comp Neurol 311:234-258.

Maunsell JH, Newsome WT (1987) Visual processing in monkey extrastriate cortex. Annu Rev Neurosci 10:363-401.

Maunsell JH, Van Essen DC (1983a) Functional properties of neurons in middle temporal visual area of the macaque monkey. I. Selectivity for stimulus direction, speed, and orientation. J Neurophysiol 49:1127-1147.

Maunsell JH, Van Essen DC (1983b) The connections of the middle temporal visual area (MT) and their relationship to a cortical hierarchy in the macaque monkey. J Neurosci 3:25632586 .

Maurer D, Lewis TL (2001) Visual acuity: the role of visual input in inducing postnatal change. Clin Neurosci Res 1:239-247.

Mayer DL, Dobson V (1982) Visual acuity development in infants and young children, as assessed by operant preferential looking. Vision Res 22:1141-1151.

McGuire BA, Gilbert CD, Rivlin PK, Wiesel TN (1991) Targets of horizontal connections in macaque primary visual cortex. J Comp Neurol 305:370-392.

McKee SP, Levi DM, Movshon JA (2003) The pattern of visual deficits in amblyopia. J Vis 3:380-405. 
Menon RS, Ogawa S, Kim SG, Ellermann JM, Merkle H, Tank DW, Ugurbil K (1992)

Functional brain mapping using magnetic resonance imaging. Signal changes accompanying visual stimulation. Invest Radiol 27 Suppl 2:S47-S53.

Menon RS, Ogawa S, Strupp JP, Ugurbil K (1997) Ocular dominance in human V1 demonstrated by functional magnetic resonance imaging. J Neurophysiol 77:2780-2787.

Merigan WH (1996) Basic visual capacities and shape discrimination after lesions of extrastriate area V4 in macaques. Vis Neurosci 13:51-60.

Merigan WH, Nealey TA, Maunsell JH (1993) Visual effects of lesions of cortical area V2 in macaques. J Neurosci 13:3180-3191.

Mizoguchi S, Suzuki Y, Kiyosawa M, Mochizuki M, Ishii K (2005) Differential activation of cerebral blood flow by stimulating amblyopic and fellow eye. Graefes Arch Clin Exp Ophthalmol.

Morland AB, Baseler HA, Hoffmann MB, Sharpe LT, Wandell BA (2001) Abnormal retinotopic representations in human visual cortex revealed by fMRI. Acta Psychol (Amst) 107:229-247.

Mosher JC, Spencer ME, Leahy RM, Lewis PS (1993) Error bounds for EEG and MEG dipole source localization. Electroencephalogr Clin Neurophysiol 86:303-321.

Mountcastle VB (1997) The columnar organization of the neocortex. Brain 120 ( Pt 4):701-722.

Movshon JA, Eggers HM, Gizzi MS, Hendrickson AE, Kiorpes L, Boothe RG (1987) Effects of early unilateral blur on the macaque's visual system. III. Physiological observations. J Neurosci 7:1340-1351.

Mussap AJ, Levi DM (1999) Orientation-based texture segmentation in strabismic amblyopia. Vision Res 39:411-418.

Norcia AM, Tyler CW, Hamer RD (1990) Development of contrast sensitivity in the human infant. Vision Res 30:1475-1486.

O'Brien DF (1982) The chemistry of vision. Science 218:961-966.

O'Kusky J, Colonnier M (1982) A laminar analysis of the number of neurons, glia, and synapses in the adult cortex (area 17) of adult macaque monkeys. J Comp Neurol 210:278-290.

Ogawa S, Lee TM, Kay AR, Tank DW (1990) Brain magnetic resonance imaging with contrast dependent on blood oxygenation. Proc Natl Acad Sci U S A 87:9868-9872.

Ogawa S, Tank W, Menon RS, Ellermann JM, Kim SG, Merkle H, Ugurbil K (1992) Intrinsic signal changes accompanying sensory stimulation: functional brain mapping with magnetic resonce imaging. Proc Natl Acad Sci U S A 89:5951-5955. 
Ohlsson J, Villarreal G, Sjostrom A, Abrahamsson M, Sjostrand J (2001) Visual acuity, residual amblyopia and ocular pathology in a screened population of 12-13-year-old children in Sweden. Acta Ophthalmol Scand 79:589-595.

Pauling L, Coryell CD (1936) The magnetic properties and structure of hemoglobin, oxyhemoglobin, and carbonmonoxyhemoglobin. Proc Natl Acad Sci U S A 22:210-216.

Pei YF, Rhodin JA (1970) The prenatal development of the mouse eye. Anat Rec 168:105-125.

Powell TP, Mountcastle VB (1959) Some aspects of the functional organization of the cortex of the postcentral gyrus of the monkey: a correlation of findings obtained in a single unit analysis with cytoarchitecture. Bull Johns Hopkins Hosp 105:133-162.

Puce A, Allison T, Asgari M, Gore JC, McCarthy G (1996) Differential sensitivity of human visual cortex to faces, letterstrings, and textures: a functional magnetic resonance imaging study. J Neurosci 16:5205-5215.

Regan D (1989) Human brain electrophysiology evoked potentials and evoked magnetic fields in science and medicine. New York: Elsevier.

Reid RC, Alonso JM (1996) The processing and encoding of information in the visual cortex. Curr Opin Neurobiol 6:475-480.

Rodieck RW, Watanabe M (1993) Survey of the morphology of macaque retinal ganglion cells that project to the pretectum, superior colliculus, and parvicellular laminae of the lateral geniculate nucleus. J Comp Neurol 338:289-303.

Schiller PH, Colby CL (1983) The responses of single cells in the lateral geniculate nucleus of the rhesus monkey to color and luminance contrast. Vision Res 23:1631-1641.

Sengpiel F, Blakemore C (1996) The neural basis of suppression and amblyopia in strabismus. Eye 10 ( Pt 2):250-258.

Sereno MI, Dale AM, Reppas JB, Kwong KK, Belliveau JW, Brady TJ, Rosen BR, Tootell RB (1995) Borders of multiple visual areas in humans revealed by functional magnetic resonance imaging. Science 268:889-893.

Shapley R, Lennie P (1985) Spatial frequency analysis in the visual system. Annu Rev Neurosci 8:547-583.

Sharma V, Levi DM, Klein SA (2000) Undercounting features and missing features: evidence for a high-level deficit in strabismic amblyopia. Nat Neurosci 3:496-501.

Sherrington CS (1906) The integrative action of the nervous system. New York: C. Scribner's sons.

Simons K (2005) Amblyopia characterization, treatment, and prophylaxis. Surv Ophthalmol 50:123-166. 
Sincich LC, Horton JC (2002) Divided by cytochrome oxidase: a map of the projections from v1 to v2 in macaques. Science 295:1734-1737.

Sincich LC, Park KF, Wohlgemuth MJ, Horton JC (2004) Bypassing V1: a direct geniculate input to area MT. Nat Neurosci 7:1123-1128.

Sireteanu R, Fronius M (1981) Naso-temporal asymmetries in human amblyopia consequence of long-term interocular suppression. Vision Res 21:1055-1063.

Sireteanu R, Fronius M (1990) Human amblyopia: structure of the visual field. Exp Brain Res 79:603-614.

Skoczenski AM, Norcia AM (1999) Development of VEP Vernier acuity and grating acuity in human infants. Invest Ophthalmol Vis Sci 40:2411-2417.

Smith EL, III, Chino YM, Ni J, Cheng H, Crawford ML, Harwerth RS (1997) Residual binocular interactions in the striate cortex of monkeys reared with abnormal binocular vision. $\mathrm{J}$ Neurophysiol 78:1353-1362.

Smith EL, III, Harwerth RS, Crawford ML (1985) Spatial contrast sensitivity deficits in monkeys produced by optically induced anisometropia. Invest Ophthalmol Vis Sci 26:330-342.

Stoerig P, Kleinschmidt A, Frahm J (1998) No visual responses in denervated V1: highresolution functional magnetic resonance imaging of a blindsight patient. Neuroreport 9:2125.

Stone J, Leicester J, Sherman SM (1973) The naso-temporal division of the monkey's retina. J Comp Neurol 150:333-348.

Stuart JA, Burian HM (1962) A study of separation difficulty. Its relationship to visual acuity in normal and amblyopic eyes. Am J Ophthalmol 53:471-477.

Thomas J (1978) Normal and amblyopic contrast sensitivity function in central and peripheral retinas. Invest Ophthalmol Vis Sci 17:746-753.

Tomita S, Kajihara S, Kondo Y, Yoshida Y, Shibata K, Kado H (1996) Influence of head model in biomagnetic source localization. Brain Topogr 8:337-340.

Tommila V, Tarkkanen A (1981) Incidence of loss of vision in the healthy eye in amblyopia. $\mathrm{Br}$ J Ophthalmol 65:575-577.

Tootell RB, Hadjikhani N (2001) Where is 'dorsal V4' in human visual cortex? Retinotopic, topographic and functional evidence. Cereb Cortex 11:298-311.

Tootell RB, Hadjikhani NK, Vanduffel W, Liu AK, Mendola JD, Sereno MI, Dale AM (1998) Functional analysis of primary visual cortex (V1) in humans. Proc Natl Acad Sci U S A 95:811-817. 
Tootell RB, Hamilton SL, Silverman MS (1984) Topography of cytochrome oxidase activity in owl monkey cortex. J Neurosci 5:2786-2800.

Tootell RB, Mendola JD, Hadjikhani NK, Ledden PJ, Liu AK, Reppas JB, Sereno MI, Dale AM (1997) Functional analysis of V3A and related areas in human visual cortex. J Neurosci 17:7060-7078.

Tootell RB, Reppas JB, Kwong KK, Malach R, Born RT, Brady TJ, Rosen BR, Belliveau JW (1995) Functional analysis of human MT and related visual cortical areas using magnetic resonance imaging. J Neurosci 15:3215-3230.

Tootell RB, Silverman MS, Switkes E, De Valois RL (1982) Deoxyglucose analysis of retinotopic organization in primate striate cortex. Science 218:902-904.

Ueno S, Iramina K (1990) Modeling and source localization of MEG activities. Brain Topogr 3:151-165.

Ungerleider LG, Haxby JV (1994) 'What' and 'where' in the human brain. Curr Opin Neurobiol 4:157-165.

Vaegan, Taylor D (1979) Critical period for deprivation amblyopia in children. Trans Ophthalmol Soc U K 99:432-439.

Van Essen DC, Anderson CH, Felleman DJ (1992) Information processing in the primate visual system: an integrated systems perspective. Science 255:419-423.

Van Essen DC, Lewis JW, Drury HA, Hadjikhani N, Tootell RB, Bakircioglu M, Miller MI (2001) Mapping visual cortex in monkeys and humans using surface-based atlases. Vision Res 41:1359-1378.

von Noorden GK, Campos EC (2001) Binocular vision and ocular motility: Theory and management of strabismus. St. Louis: Mosby.

von Noorden GK, Crawford ML (1979) The sensitive period. Trans Ophthalmol Soc U K 99:442-446.

von Noorden GK, Maumenee E (1968) Clinical observations on stimulus-deprivation amblyopia (amblyopia ex anopsia). Am J Ophthalmol 65:220-224.

Wald G, Burian HM (1944) The dissociation of form vision and light perception in strabismic amblyopia. Am J Ophthalmol 27:950-963.

Wassle H, Boycott BB (1991) Functional architecture of the mammalian retina. Physiol Rev 71:447-480.

Wiesel TN, Hubel DH (1963) Single-cell responses in striate cortex of kittens deprived of vision in one eye. J Neurophysiol 26:1003-1017. 
Wiesel TN, Hubel DH (1966) Spatial and chromatic interactions in the lateral geniculate body of the rhesus monkey. J Neurophysiol 29:1115-1156.

Wilson HR (1988) Development of spatiotemporal mechanisms in infant vision. Vision Res 28:611-628.

Yamada E, Ishikawa T (1965) The fine structure of the horizontal cells in some vertebrate retinae. Cold Spring Harb Symp Quant Biol 30:383-392.

Zeki SM (1973) Colour coding in rhesus monkey prestriate cortex. Brain Res 53:422-427. 


\section{CHAPTER 2: Objectives and Rationales}


This investigation was focused on using functional magnetic resonance imaging (fMRI) to better characterize the neural basis of amblyopia in humans. Amblyopia is an important and clinically relevant disorder of visual development, which results in significant visual impairment for $1-3 \%$ of the population. The experiments in this study were designed to meet three separate objectives; the results of these experiments are detailed in Chapters 3, 4, and 5. This chapter will introduce the three objectives and present brief rationales for the experiments.

\subsection{Objective 1- To determine the cortical retinotopic organization in children with normal vision.}

Although the retinotopic organization of the visual cortex has been well studied in adults, there has been no known study of the cortical retinotopic organization in children. Visual cortex requires visual experience to mature in the first decade after birth, and only reaches the adult state between the ends of the first and second decades. Furthermore, the abnormal developmental processes that characterize amblyopia can still be reversed with treatment into the second decade. Functional imaging could be used to evaluate and tailor treatment in children with amblyopia. Therefore, it is important to establish a functional baseline for comparison with normal children. Normal children might have adult-like cortical retinotopic organization, but could also be expected to show differences due to developmental lags in neural or hemodynamic components.

Methodologically, it is also useful to extend adult fMRI techniques to pediatric groups in order to develop better age-appropriate paradigms and tasks. Child acclimation to the scanner environment and training for vision and fMRI testing are two hurdles faced by the vision scientist who is committed to studying children and visual development using fMRI. Ideally, the lessons learned with older children could be refined and applied to progressively younger populations (to a limit), so that vision and plasticity could be studied throughout development. Chapter 3 discusses the application of traditional fMRI retinotopic mapping techniques to children with normal vision. 


\subsection{Objective 2- To determine the cortical retinotopic organization in adults with amblyopia.}

Amblyopia is characterized by an impairment of acuity and contrast sensitivity, which is particularly acute for central vision. This impairment has been studied extensively using psychophysics, yet physiologic measurements in humans have been limited through the years to recordings of evoked potentials from the scalp. These recordings have provided great insight into the functionality of the amblyopic visual system, but have been unable to directly correlate this functionality with anatomy. Recently, functional neuroimaging techniques like positron emission tomography (PET) and fMRI have begun to allow investigation of the anatomical neural basis for the psychophysical deficits, as well as comparison to well-established animal models of amblyopia. However, no other study has yet examined the cortical organization associated with the central visual deficit in amblyopia. Chapter 4 discusses the monocular retinotopic organization found in adults with amblyopia compared with control subjects.

\subsection{Objective 3- To determine the cortical retinotopic organization in children with amblyopia.}

Children with amblyopia are the most clinically relevant of these three groups. Treatment of amblyopia is only effective in childhood, while the visual system retains its innate plasticity. Therefore, children with amblyopia are most likely to benefit from the results of this study, which could be used to advise better treatment management and to evaluate alternatives to the traditional patching therapy. This represents the first known attempt to systematically study the retinotopic organization of visual cortex in children with amblyopia. Chapter 5 discusses the extension of monocular retinotopic mapping to amblyopic children, comparing their results with both normal children and amblyopic adults. 


\section{CHAPTER 3: Retinotopic Organization in Children Measured with fMRI}

This chapter was published in Journal of Vision, June 18, 2004, 4(6):509-523. 


\subsection{Abstract}

Many measures of visual function reach adult levels by about age 5, but some visual abilities continue to develop throughout adolescence. Little is known about the underlying functional anatomy of visual cortex in human infants or children. We used fMRI to measure the retinotopic organization of visual cortex in 15 children aged 7-12 years. Overall, we obtained adult-like patterns for most children tested. We found that significant head motion accounted for poor quality maps in a few tested children who were excluded from further analysis. When the maps from 10 children were compared with those obtained from 10 adults, the magnitude of retinotopic signals in visual areas $\mathrm{V} 1, \mathrm{~V} 2, \mathrm{~V} 3, \mathrm{~V} 3 \mathrm{~A}, \mathrm{VP}$, and $\mathrm{V} 4 \mathrm{v}$ was essentially the same between children and adults. Furthermore, one measure of intra-area organization, the cortical magnification function, did not significantly differ between adults and children for V1 or V2. However, quantitative analysis of visual area size revealed some significant differences beyond V1. Adults had larger extrastriate areas (V2, V3, VP, and V4v), when measured absolutely or as a proportion of the entire cortical sheet. We found that the extent and laterality of retinotopic signals beyond these classically defined areas, in parietal and lateral occipital cortex, showed some differences between adults and children. These data serve as a useful reference for studies of higher cognitive function in pediatric populations and for studies of children with vision disorders, such as amblyopia. 


\subsection{Introduction}

Psychophysical measurements in humans have established that the visual system depends extensively on postnatal experience to guide developmental mechanisms and eventually reach a mature state. Although perhaps the most dramatic improvements in performance occur in the first year of life, some abilities require many years of visual experience to achieve the adult-like state. For example, some measures of basic vision, such as grating acuity, do not reach adult values before age 6 . Temporal contrast sensitivity at high frequencies $(20$ and $30 \mathrm{~Hz})$ and critical flicker fusion frequency are adult-like at age 4, although contrast sensitivity for lower temporal frequencies and static gratings matures between ages 4 and 7 (Ellemberg et al., 1999). Even later maturation of several visual functions that require global integration across distance in the visual field has been recently documented. For example, texture segregation does not reach adult levels until between ages 14 and 18 (Sireteanu, 2000). Contour integration has been shown to have a protracted development when tested with oriented Gabor element textures, and with the Ebbinghaus illusion (Kovacs et al., 1999; Kovacs, 2000). Finally, Vernier hyperacuity undergoes a steep improvement during childhood until adult levels are reached at age 14 (Skoczenski and Norcia, 2002).

Much less is known about the physiological maturation of the visual system during childhood. One of the few available techniques is noninvasive electrical recording from the scalp, and such data indicate ongoing neural development through childhood. For example, the checkerboard onset evoked potential does not obtain its adult form before puberty (Ossenblok et al., 1994). Evoked potentials and visual performance measures sometimes show nice parallels, but not always (Regan and Spekreijse, 1986; Tyschen, 1992). Interestingly, some evidence suggests that the responses of visual neurons are often more mature than behavioral measures would predict (Kiorpes and Movshon, 2003), although the reasons for this discrepancy are still unclear.

The advent of noninvasive functional neuroimaging (fMRI) provides new opportunities to improve understanding of the functional neuroanatomy of humans in health and in disease. Although the source of the measured signal is hemodynamic, not neural, it is coupled to neural activity (Logothetis et al., 2001) with an impressive spatial (2-4 mm) and temporal (1-2 s) resolution. These techniques can be applied to pediatric populations, and have been done so 
increasingly (Casey et al., 1995; Kwon et al., 2002; Temple et al., 2003; Turkeltaub et al., 2003). Most efforts have focused on cognitive functions, such as reading ability and memory. The majority of such studies provide stimuli through the visual modality. Therefore, it is important to understand the state of maturity of early visual areas in this age range for better interpretation of cognitive studies, as well as to directly investigate human visual system development.

Retinotopic organization of human visual cortex has been mapped using functional magnetic resonance imaging (fMRI) in adults. Multiple visual areas have been shown to exist, each with its own representation of visual space (Sereno et al., 1995; DeYoe et al., 1996; Engel et al., 1997). This type of mapping of visual cortex is important for several reasons. 1. The technique allows for rapid delineation of a large expanse of visual cortex. 2. The boundaries of several visual areas can be defined by the representations of the horizontal and vertical meridians of visual space. Such objective boundaries guide the creation of regions of interest that can be applied in a statistically independent way to the results of other experiments. 3. By mapping a fundamental and continuously changing variable across the cortical sheet, significant information can be acquired about intra-areal function.

These powerful noninvasive methods for mapping retinotopic function have not previously been extended to children. Here we show that visually normal children have essentially achieved an adult-like pattern of many retinotopic visual areas mapped with functional magnetic resonance techniques, at least by age 9 . However, quantitative differences were detected between the adult and child groups in the size of visual areas. To extend our results beyond these well-known areas, we also employed new cortical surface-based methods for achieving inter-subject averaging. Different patterns of activation in parietal and lateral occipital cortices were observed. These data can serve as an important baseline from which to compare the retinotopic maps of normal children to those with visual disorders. 


\subsection{Materials and Methods}

\subsubsection{Subjects}

We studied 10 adults aged 21 to 30 years (6 male, 4 female) and 15 children aged 7 to 12 years ( 7 male, 8 female). Seven child subjects were less than 10 years and 8 were greater than or equal to 10 years. After excluding from analysis subjects exhibiting significant head motion, 10 subjects remained, and their ages were 9, 9, 10, 10, 10, 11, 12, 12, 12, and 12 years. With regard to the handedness of our subjects, $9 / 10$ adults were right-handed and 8/10 children were righthanded.

Our subjects were recruited from the local community surrounding West Virginia University (WVU). Informed consent was obtained from all subjects in a project approved by the WVU Institutional Review Board for the Protection of Human Subjects (protocol \#14788).

\subsubsection{Cortical Surface Reconstruction}

Surface reconstructions of each subject's cerebral cortex were generated from highresolution anatomical images obtained in a General Electric 1.5 Tesla MR scan session separate from the retinotopic mapping experiments. Previously validated techniques (Dale et al., 1999; Fischl et al., 1999a) were employed. Briefly, brain reconstruction was begun by collecting whole-head 3D fast spoiled grass gradient echo (FSPGR) scans, optimized for contrast between gray and white matter, for each subject. Specific parameters were fast IR prep (prep time $=300$ $\mathrm{ms}), \mathrm{TE}=1.9 \mathrm{~ms}$, flip angle $=20 \mathrm{deg}, \mathrm{FOV}=24 \mathrm{~cm}$, axial slices, $256 \times 256$ matrix, resolution $0.94 \times 0.94 \times 1.2 \mathrm{~mm}$.

The surface reconstructions were created using the FreeSurfer software package available at http://www. nmr.mgh.harvard.edu/freesurfer. Voxels containing white matter in an intensitynormalized volume were labeled using an anisotropic planar filter. A region-growing algorithm was then used to ensure that each cortical hemisphere was represented by a single connected component with no interior holes. The surfaces of these components were tessellated $(\sim 150,000$ vertices), refined against the MRI data using a deformable template technique, and manually inspected for topological defects (i.e., departures from spherical topology). Automated 
techniques for optimizing topological correctness while maintaining geometrical accuracy were employed (Fischl et al., 2001).

In a separate step, the cortical surface was computed by expanding the gray/white surface by $3 \mathrm{~mm}$ and refining it against the FSPGR MR images. The sampled functional signal included most of cortical gray matter, but it was centered just above the gray/white boundary to avoid the pial surface where macrovascular fMRI artifacts are greatest, and to ensure that functional signals were assigned to the correct sulcal bank. The surface reconstruction of each subject's brain was "inflated" by an iterative algorithm that reduced local curvature while approximately preserving local areas and angles by minimizing metric distortion.

Once a cortical surface is reconstructed, it becomes possible to calculate the surface area of the entire cortical sheet, or of smaller cortical areas defined as regions of interest (ROI). Although the ROIs can be drawn (i.e., defined) on the flattened surface, the ROIs are mapped back to the folded cortical surface when measurements are made, avoiding the areal distortion created by inflation and flattening. Measures of surface area are made at the gray/white boundary, as this best represents the location of recorded functional signals. We compute the area associated with the polygonal surface model, which is very densely sampled. The area of each triangular vertex is approximately $0.5 \mathrm{~mm}^{2}$. The area of an ROI is the sum of the area of $n$ triangles. Due to the inherent difficulty of segmentation of white from gray matter, segmentation is a potential source of error in the reconstructions. However, the accuracy of the programs has been validated (e.g., by test-retest comparisons) for estimates of cortical thickness (Fischl and Dale, 2000; Rosas et al., 2002). Furthermore, each reconstruction is manually inspected for errors.

\subsubsection{Functional Magnetic Resonance Imaging}

Subjects were scanned in a GE 1.5 Tesla MR scanner using techniques described previously (Mendola et al., 1999). After a sagittal localizing scan was obtained, a T1-weighted inversion recovery sequence ( $\mathrm{TR}=400 \mathrm{~ms}$ ) was used to acquire 20 interleaved 4-mm slices with $0.86 \times 0.86 \mathrm{~mm}$ in-plane resolution, oriented perpendicular to the calcarine sulcus. These anatomical scans were later used to register the functional scans to the FSPGR slices that were used to define the cortical surface. 
The next step was to acquire multiple functional scans using the same slice prescription selected in the anatomical scans, but with $3.44 \times 3.44 \mathrm{~mm}$ in-plane resolution. Functional signals reflecting neural activity via local oxygen consumption and blood flow were acquired (Kwong et al., 1992; Ogawa et al., 1992) using a spiral gradient echo sequence (TE $=40 \mathrm{ms,} \mathrm{TR}$ $=4000 \mathrm{~ms}$, flip angle $=65 \mathrm{deg}, \mathrm{FOV}=22 \mathrm{~cm}$, matrix $64 \times 64$ (Glover, 1999).

For the adults, functional scans had a duration of $8 \mathrm{~min}$ and $32 \mathrm{~s}$, and 128 time points were collected from each slice in all scans. Four scans of this type were administered in one session, two scans for eccentricity and two scans for polar angle. For the children, functional scans had a duration of $4 \mathrm{~min}$ and $16 \mathrm{~s}$, and 64 time points were collected from each slice in all scans. Six scans of this type were administered in one session, three scans for eccentricity and three scans for polar angle. The entire scanning procedure typically lasted about $2 \mathrm{hr}$.

Head movement (within and between scans) was minimized by the use of one of two methods. Most subjects used a bite bar, in which subjects stabilized their jaw in a rigid, deep individual dental impression, mounted in an adjustable frame. For children who were not comfortable using a bite bar, we used a rigid chin cup that greatly restricted movements in all planes. Although we did not monitor eye movements, the good quality of our retinotopic maps indicates adequate fixation during the functional scans. Our use of radial motion and symmetric stimuli also helps to minimize translational eye position drifts.

\subsubsection{Visual Stimuli}

During the fMRI experiments, the visual stimuli were generated by a Silicon Graphics O2 computer with an output resolution of $640 \times 480$ pixels. The video output was converted to a 60 $\mathrm{Hz}$ interlaced composite S-VHS signal, which served as input to an Epson Powerlite 500c LCD projector. The projector's image passed into the bore of the magnet, and appeared on a paper rear-projection screen in front of the subject. The subjects viewed the screen by looking straight up at a mirror placed at an approximately 45-deg angle to both the screen and the subject's line of sight. The stimuli subtended an area of about 25-deg horizontal by 15-deg vertical.

The cortical representation of retinotopic visual space was mapped with a phase-encoded design in which the cardinal axes of space (eccentricity and polar angle) were mapped separately (Engel et al., 1994; DeYoe et al., 1996; Engel et al., 1997). The stimuli consisted of high- 
contrast, chromatic, flickering checkerboard patterns of two specific types. The "rotating wedge" stimulus would sweep through polar angles much like a hand on a clock, and the "expanding ring" stimulus mapped eccentricity by starting from the center of the visual field and expanding outward (Fig. 3-1). Eccentricity stimuli traversed space with a logarithmic transformation, as has been used previously (Sereno et al., 1995; Tootell et al., 1997). Both stimuli attempted to compensate for the cortical magnification factor by increasing in size as they approach the periphery. These phase-encoded stimuli always used a cycle length of $64 \mathrm{~s}$, which corresponds to 8 cycles per scan for adults and 4 cycles per scan for children. Adults were shown 16 cycles of each stimulus type, whereas children were shown 12 cycles. The lower number of cycles was used for the children due to concerns about their stamina for accurate fixation and minimal head motion. However, the number of cycles was equated between groups at the subsequent analysis stage by excluding a portion of the adult data.

A central fixation mark was present at all times for all stimuli. Subjects were clearly instructed to maintain fixation on this mark at all times during an fMRI scan. Subjects were also instructed to perform a task monitoring the appearance of the fixation point to aid fixation stability. The fixation point briefly changed color from white to red with an inter-trial interval that randomly ranged from 4-32 s (in 4-s multiples) during the course of an fMRI scan, and the subjects pressed a key upon detecting such a change. The children were able to perform this task at a very accurate level (mean $=98 \%$ correct $)$.

\subsubsection{Statistical Analysis}

\subsubsection{Individual Subject Analysis}

The functional analysis was completed using the FS-FAST software tools freely available at ftp:/ftp.nmr. mgh.harvard.edu/pub/flat/fmri-analysis. A brief description of the processing steps follows. For each subject, raw MR images were first motion-corrected using an iterated, linearized weighted least-squares method, through the FS-FAST implementation of the AFNI 3dvolreg algorithm (Cox and Jesmanowicz, 1999), and then intensity-normalized using the average in-brain voxel intensity. The residual, uncorrectable head motion was measured as the root mean square (RMS) difference between motion-corrected output and a target volume defined from the middle time point of each scan. This value was computed for each subject, and 
values exceeding a RMS of 60 , which corresponded to the grossly observed image artifacts, were used as a criterion for exclusion of some of the children $(5 / 15)$ from further processing. For the remaining 10 subjects, the average corrected motion in all three planes was quantified as the vector magnitude of translational motion.

After the preprocessing steps, a fast Fourier analysis was conducted on the time series of each voxel to statistically correlate retinotopic stimulus location with visual cortical anatomy. To equate the children and adults for data analysis, only the first 12 cycles were analyzed for the adults. This analysis rejects low frequencies due to subject head motion or baseline drift and extracts functional signals in the form of magnitude and phase relative to the stimulus cycle frequency ( 8 or 4 cycles per scan; period $=64 \mathrm{~s}$ ). Signal magnitude reflects the degree of retinotopic specificity, which can be low due to either a lack of visually induced response or to an equal response to all retinotopic locations. The phase component of the signal was used to code retinotopic location. Activation significance $(F)$ values were computed for each voxel by using a comparison between the Fourier domain amplitude at the stimulation frequency and the average amplitude at other (non-harmonic) frequencies. For these types of calculations, the phase/magnitude data are converted from polar coordinates to the equivalent data in rectilinear coordinates (i.e., complex numbers with real and imaginary components). The $F$ statistic accommodates this multivariate analysis. The $F$ statistic is computed as the sum of the squared real and imaginary signal components divided by the noise variance. Finally, a registration procedure within Freesurfer was used to align (in all 3 planes) the T1-contrast anatomical images collected in each functional session with the cortical surface model. The same registration matrix was then applied to the functional images to view the results on the cortical surface.

The data from the paired eccentricity and polar angle scans were combined to yield field sign maps. The field sign maps the polarity of the visual field representation as either similar to the actual visual field geometry or mirror symmetrical to it. The field sign for each cortical area was objectively calculated from the vector product of the constituent phase-encoded maps of polar angle and eccentricity as in Sereno et al. (1995). Visual area naming conventions are as described in Tootell et al. (1997), and are consistent with previous studies. The superior portions of V1, V2, and V3 contain representations of the contralateral lower visual field, whereas the inferior portions of $\mathrm{V} 1, \mathrm{~V} 2, \mathrm{VP}$, and $\mathrm{V} 4 \mathrm{~V}$ represent the contralateral upper visual field. V3A represents both the lower and upper contralateral field. Areas V1, V2, VP, V3, V3A, and V4v 
are classical retinotopic areas that have been described previously. We did not attempt to explicitly identify retinotopic areas anterior to these areas. There are additional retinotopic areas, including V7 and V8, whose cruder retinotopy has been demonstrated only with high-field scanning (Hadjikhani et al., 1998; Tootell et al., 1998a; Wade et al., 2002). Also, there is still debate regarding the appropriate definition of visual areas in this region. This fringe retinotopy region has also been shown to be activated by both left and right visual fields (Tootell et al., 1998b). Thus, the current evidence suggests that areas V7 and V8 lie near the end of a continuum of decreasing retinotopy and increasing receptive field sizes.

\subsubsection{Region-of-Interest Analysis}

To generate ROIs specific to a given visual area, patches of flattened cortex that corresponded to each retinotopic area were defined based on the retinotopic field sign map for each subject (Mendola et al., 1999). These objectively defined borders were available for visual areas V1, V2 (superior and inferior), V3, VP, V3A, and V4v. The eccentricity range of these ROIs was approximately $1-15^{\circ}$ visual angle. Specifically, the vertical eccentricity was $7.5^{\circ}$ and the horizontal eccentricity was $12.5^{\circ}$, making the maximum (diagonal) eccentricity $15^{\circ}$. The outer extent of the ROI was based on the actual activation present. Because the resolution of these maps is typically too low to measure the extremely high cortical magnification factor in the central foveal representation, the ROIs did not include the entire foveal representation. The minimum eccentricity value varied slightly from subject to subject, but was between 1-2 deg for most (see Fig. 3-5). For each subject, the Freesurfer software program was used to draw the outline of each visual area for each hemisphere. In this way, we defined the boundaries of six visual areas for the 10 adults and 10 children with interpretable field sign maps. For visual area V2, an ROI was drawn for the superior/dorsal branch and another for the inferior/ventral branch (see Fig. 3-2), although the results for these branches were combined in subsequent analysis. For all RO1s except V1, the flattened cortex was used for visualization. For V1, the inflated cortex was used for visualization so that the split along V1 in the flattened view did not interfere with definition. Regardless of the view chosen for visualization, all ROIs exist in native (folded) coordinates when measurements are made.

\subsubsection{Cortical Magnification Factor Analysis}


To further compare the internal organization of the V1 and V2 retinotopic map in children and adults, we computed the cortical magnification function as has been done previously by others for adult subjects (Sereno et al., 1995; Engel et al., 1997; Duncan and Boynton, 2003). This first required definition of an approximately iso-polar ROI along the representation of the horizontal meridian within V1 for every subject. This was done by applying a filter to the V1 ROI to accept voxels with polar angle phase values at the horizontal meridian $(90 \mathrm{deg}) \pm 15 \mathrm{deg}$. We then determined the eccentricity phase values of all voxels in this ROI. These voxels were sorted according to distance from a reference point at the occipital pole defined in every subject. The distance was computed as the arc distance along the inflated cortical surface in spherical space, allowing us to express distance along the cortical sheet, regardless of cortical folding pattern. The distance of each voxel was then plotted against its eccentricity phase value (or the equivalent degrees of visual angle). Finally, this curve was fit with an exponential function that best fit the least square data. Specifically,

$$
y=y_{0} e^{k x}
$$

where $x$ is the cortical distance and $y$ is the eccentricity. The process for V2 was equivalent, except that the 30-deg filter was centered on oblique polar angles (135 deg for V2v and $45 \mathrm{deg}$ for V2d), so as to fall along the center of those areas. One adult subject and one child subject, with poor quality polar angle maps, did not satisfy our criteria of least squares $R^{2}$ fit greater than 0.5 , and were excluded from this analysis.

\subsubsection{Across-Subjects Analysis}

Directly averaging fMRI data across subjects is an inherently difficult task, due to the differences in the size, shape, and even functional organization of subjects' individual brains. Using common spaces, such as the traditional neurosurgical Talairach space (Talairach and Tournoux, 1988) or even more modern versions such as the space defined by 152 adult subjects at the Montreal Neurological Institute (Evans et al., 1993), introduces a large amount of spatial blurring into the data set. Typical variability between presumably homologous points in subjects is of the order of $1 \mathrm{~cm}$ in Talairach space. Given that individual visual areas have a (flattened) width of about $1 \mathrm{~cm}$, such averaging procedures seriously degrade the quality of retinotopic maps. Nonetheless, across-subject averaging remains a desirable goal. Specifically, to compare 
the retinotopic maps obtained beyond the 6 defined visual areas in parietal and temporal cortex, we need a strategy that does not require ROIs.

Thus, to compare the adult and child groups, we used a new technique for across-subject analysis, adapted to the folding pattern of each subject, which is significantly more accurate. Each adult's inflated cortical surface was first registered to a standardized average inflated unitsphere based on alignment of gyral and sulcal patterns using techniques described elsewhere (Fischl et al., 1999b). To combine functional data across individuals for a group comparison, Fvalue data sets were then resampled into spherical space and subsequently averaged across subjects using a fixed-effects model (i.e., $F$ ratio numerator is summed real plus imaginary components squared, and denominator is the summed noise variance divided by number of subjects). Extension of these methods to random effects is desirable, but will require accounting for multiple comparisons on the surface, and clustering approaches may not be appropriate for retinotopic data. Finally, the average $F$-value map was painted (via the spherical transformation) onto the inflated surface of one adult. Separate maps were created for the eccentricity and polar angle stimulus in each hemisphere. The entire analysis was subsequently repeated for the group of children.

It should be noted that the standardized spherical template currently available in Freesurfer was created with adult brains. Therefore, the children's brains require somewhat more distortion than the adult's when placed into spherical space. To document this, the Jacobian determinant of each vertex can be computed to indicate how much the transformation expands or shrinks each vertex in the brain (expansion results in values greater than 1, whereas contraction produces values less than 1). For the entire right hemisphere, the mean adult variance was between 1 and 1.5, whereas the child variance was 2.1. Left hemisphere values were 1.4 and 2.1. The comparison of variances across groups with a $t$-test approached significance $(p=0.06$ and $p=0.05$, respectively). 


\subsection{Results}

\subsubsection{Individual Phase-Encoded Maps}

Maps of eccentricity and of polar angle were obtained as has been reported previously for adult subjects (Sereno et al., 1995). Eccentricity maps showed the well-known organization, with central vision represented at the pole and peripheral vision more anteriorly. Polar angle maps were obtained with vertical meridian representation separating V1 and V2, and horizontal meridian separating V2 from V3 and VP. V3 and VP were separated from V3A and V4v by lower and upper vertical meridian, respectively. Similar maps were obtained from the children.

To better localize the boundaries between areas, we used the eccentricity and polar angle data to compute a field sign map (Sereno et al., 1995). All adult subjects produced maps of sufficient quality to identify the six retinotopic areas described for humans (V1, V2, V3, VP, $\mathrm{V} 3 \mathrm{~A}$, and $\mathrm{V} 4 \mathrm{v})$. We obtained interpretable maps in 10 of 15 children, and these maps were qualitatively similar to those seen in adult subjects. An example is shown of one 11-year-old subject (Fig. 3-2).

Observation during the experiments, subjective reports from the children, and head motion artifacts indicated that head motion likely contributed to the poor quality of maps in the other five subjects. Four of these subjects were among the youngest children, 7-9 years old. These subjects were excluded from further analysis.

\subsubsection{Head Motion}

To quantify the head motion in each of the experimental scans, we used the motioncorrection algorithm in AFNI. For each subject we extracted the average motion in all three directions as the vector magnitude of translational motion. This value ranged from $0.2-0.5 \mathrm{~mm}$ for adults and 0.3-2.0 mm for children (Fig. 3-3). Pearson product correlations were performed to look for any consistent relation between age and corrected head motion, especially in the young group. We did not find a significant correlation in the child group $\left(R^{2}=0.07\right)$ or the adult group $\left(R^{2}=0.34\right)$. 


\subsubsection{Region-of-Interest Analysis for Across-Group Comparisons}

Thus far, qualitative inspection of the retinotopic maps from representative children and adults did not suggest consistent differences between groups. However, it is important to be able to quantify measures from individual visual areas that can be averaged across subjects to address any systematic group differences. For the 10 adults and 10 children with interpretable field sign maps, regions of interest were created for the six visual areas (V1, V2, V3, V3A, VP, and V4v). A seventh ROI entitled "All" was defined to include all six visual areas. These ROIs were used to separately extract from our data the average fMRI signal Fourier magnitude of all voxels located in each of the six visual areas. We also directly calculated the surface area of visual area ROIs as described in Section 3.3.2.

\subsubsection{Measures of Signal Magnitude}

The average Fourier magnitude was calculated for all voxels in a given visual area, for the left and the right hemispheres separately. The results are listed in Tables 1 and 2. Direct comparison between evoked fMRI signal in each area for adults and children was performed with $t$-tests. For the polar angle stimulus, only the right VP was significantly different, having a larger value in adults. In the next section, we consider this difference in relation to the size of the visual areas.

Pearson product correlations were also performed to look for any developmental relationship between age and Fourier magnitude, as well as between corrected head motion and signal magnitude, especially in the young group. There were no significant correlations between magnitude and head motion for either group. There were several visual areas that showed a significant correlation between magnitude and age within the child group. However, the magnitude was negatively correlated with age, and may be due, in part, to the unusual coincidence that four of our youngest children were scanned on the same day. Also, the areas showing this correlation were not consistent between hemi-spheres. No correlations with age were significant for the adult group.

\subsubsection{Measures of Visual Area Size}


For each subject in both groups, the size of the six visual areas was analyzed in terms of absolute surface area in $\mathrm{mm}^{2}$, and as a relative proportion of the total cortical sheet. The adult group and the child group were then directly compared. The results are listed in Tables 3 and 4.

The size of visual areas in both adults and children covers a range of about $400-700 \mathrm{~mm}^{2}$, except for V1 and V2, which ranged from $800-1200 \mathrm{~mm}^{2}$. The results indicated some small but significant differences between the adults and children. Adults showed a slightly larger extent of visual areas $\mathrm{V} 2, \mathrm{~V} 3$, and $\mathrm{V} 4 \mathrm{v}$ in the left hemisphere. In the right hemisphere, visual areas V2, $\mathrm{V} 3$, and VP were significantly larger in adults. In contrast, there was no difference in the extent of V1 between groups.

One concern with the use of an absolute measure of visual area ROI size is that children's brains might be globally smaller than adults' brains. Hence, we compared cortical surface area for the entire neocortical reconstruction of each hemisphere between children and adults. The left and right hemisphere reconstructions yielded a mean measure of 81,734 and $81,128 \mathrm{~mm}^{2}$ for the children, and 87,009 and $86,011 \mathrm{~mm}^{2}$ for adults. The adult's brains were larger than the children's brains, but the effect was not significant $(p=0.12$ for left hemisphere; $p=0.13$ for right hemisphere). Nevertheless, given the obvious developmental trend, a measure of ROI size relative to the total neocortical sheet may be a valuable measure (Tables 3 and 4). Overall, individual visual areas range from $0.5-1.5 \%$ of the cortical sheet, and all six visual areas combined occupy about $5 \%$ of the neocortex in one hemisphere. When the adults and children were compared, the results were highly consistent with the comparisons of absolute size in that similar extrastriate areas proved to be slightly larger in adults. The total proportional size of the visual areas of both hemispheres in children and adults is shown graphically (Fig. 3-4).

Pearson product correlations were performed to look for any consistent developmental relation between age and surface area, especially in the young group. However, we found no significant correlations for the child or the adult group.

It is interesting to consider the fact that the mean level of Fourier magnitude did not differ between children and adults, yet we observed a correlation between Fourier magnitude and children's age. In contrast, mean areal size did differ between children and adults, yet no correlation was found between size and age. This suggests that the Fourier magnitude measure was more variable than areal size, and this is indeed the case. To document this efficiently, we calculated the coefficient of variance $(\mathrm{CV})$ as the SD divided by the mean. Fourier magnitude 
measures for children's visual areas had a CV in the range of 50-55\%; adults produced values ranging from $35-45 \%$. Measures of areal size (absolute and normalized) had a CV in the range of $15-30 \%$ for both children and adults.

It is well known in the neuroimaging field that magnitude of fMRI signals cannot typically be separated from the extent of activation. However, in the case of phase-encoded retinotopy, the situation is different. Signal magnitude is not directly used to determine the size of the visual areas, although a minimal magnitude is required to carry the phase-encoded signals. The preceding paragraph certainly documents that these two variables showed different patterns in our results. For a few visual areas, we did observe a significant correlation between Fourier magnitude and area size, but these did not dominate the data set and showed no clear consistency. Specifically, for children, normalized area was correlated with magnitude for left $\mathrm{V} 4 \mathrm{v}$ and right V2 for eccentricity, and right All for polar angle. For adults, normalized area was correlated with magnitude for left VP for eccentricity, and left V4v for polar angle.

\subsubsection{Cortical Magnification Function for Across-Group Comparisons}

In addition to measures of Fourier magnitude, our methods provide information about the phase of the MRI signal for each voxel within a visual area. A smooth progression of phase values is produced by the eccentricity and polar angle stimuli (the retinotopic map). An important feature of this map is the amount of cortex devoted to representing a unit of visual space, and this can be plotted as a cortical magnification function. We computed the cortical magnification function for areas V1 and V2 in children and adults. For each subject, we plotted stimulus eccentricity versus cortical distance from the occipital pole along the horizontal meridian in V1.

The curves from each subject were well fit by exponential functions $\left(R^{2}>0.5\right)$. Moreover, the average adult and children curves were fit with $R^{2}$ values of 0.94 and 0.96 , respectively (Fig. 3-5). The mean fitted exponent was 0.074 for the adults and 0.071 for the children. The two subject groups did not have a different distribution of exponential values. This was true both when the fit was done after placing data in 1-deg bins $(p=0.66)$ and in the case of the non-binned data $(p=0.96)$. Thus, our data indicate no difference in the precise 
retinotopic mapping function, although it can be seen that the variance of the child group was somewhat greater.

For V2, we measured the cortical magnification function for both the ventral and dorsal branch, V2v and V2d (Fig. 3-6). The results were similar to those for V1. Specifically, for V2V we obtained $R^{2}$ values of 0.98 and 0.97 for adults and children. The mean fitted exponential value is 0.073 for the adults and 0.057 for the children. For V2d, $R^{2}$ values are 0.96 and 0.92 for adults and children. The mean fitted exponential value is 0.081 for the adults and 0.080 for the children. The two subject groups do not have a different distribution of exponential values for $\mathrm{V} 2 \mathrm{~V}(p=0.66)$ or $\mathrm{V} 2 \mathrm{~d}(p=0.99)$. With regard to any differences between the exponential function for $\mathrm{V} 2 \mathrm{~V}$ and $\mathrm{V} 2 \mathrm{~d}$, there is a significant difference for the children $(p=0.01)$ with $\mathrm{V} 2 \mathrm{~d}$ showing a steeper slope than V2v. The same trend is observed in adults.

\subsubsection{Cortical Surface Averaging for Across-Group Comparisons}

The parietal, lateral occipital, and temporal cortical regions are not thought to contain precise retinotopic maps, but cruder retinotopic biases may, in fact, exist (Malach et al., 2002; Hasson et al., 2002). To compare phase-encoded retinotopic signals in these regions, we could not use the ROI strategy because the field sign maps do not extend into these cortices, and thus do not provide a method for identifying visual area boundaries. Using anatomically defined ROIs to directly compare children and adults was rejected due to uncertainty regarding homologous points in the cortex of these two groups. The two groups could nevertheless be compared by creating maps that show the average activation pattern for all children and for all adults.

Specifically, we used a new technique for performing across-subject averaging (Fischl et al., 1999b). This new solution is based on a spherical surface template, and provides significantly improved accuracy over volume-template techniques. This method uses the cortical surface reconstructions that we made for every subject, is based on localizing position relative to the $2 \mathrm{D}$ cortical sheet, and is adapted to the folding pattern of each individual subject. We thus made across-subject averages of the statistical measure of Fourier magnitude based on the Fstatistic. Average F-statistic maps were produced for the adult group and the children's group. The results for the eccentricity stimulus indicate a larger extent of signal in the adult subjects in 
parietal cortex (Fig. 3-7). Inspection of the individual data revealed that this was a consistent trend in the groups, for both hemispheres. Seven out of 10 adults showed some activation in the middle and/or anterior extent of the intraparietal sulcus, whereas only three children passed these criteria.

The results for the polar angle stimulus showed that the children and adults differed in the direction of their hemispheric laterality (Fig. 3-8). The adult group produced more activity in the right hemisphere, whereas the children displayed more activity in the left hemisphere. This finding was consistent at the level of individual subjects. Five adults and six children showed asymmetric activation in the direction stated above, with only one child and adult showing the opposite. This one adult was left-handed, but the child was right-handed.

In contrast to these differences, the maps of Fourier magnitude $F$-statistic in adults and children appeared qualitatively similar in the ventral temporal cortices of both hemispheres (Fig. 3-9). 


\subsection{Discussion}

The results of this study provide a quantitative comparison of retinotopic mapping in adults and children. Retinotopic organization in children older than 9 years and in adults is qualitatively similar, and six classically reported visual areas could be readily identified (V1, V2, V3, V3A, VP, and V4v). Small differences in regional signal magnitude and areal size were documented, and suggest some developmental trends. According to our experience, children younger than 9 years may require more training due to greater head motion and less attention to task. Extra training may improve success rates for this group.

Although motion artifact appeared to be the major determinant of poor fMRI maps in our youngest subjects (aged 7 and 8 years), we cannot completely exclude immaturity at the neural or hemodynamic levels. The basic mechanism of image contrast in fMRI is known (called blood oxygenation level dependent [BOLD]), but a detailed understanding of the coupling between changes in neural activity and changes in blood oxygenation and flow has not yet been achieved. It is not known if this mechanism changes during the course of development. Some results have indicated a negative BOLD response in the visual cortex of sedated infants, raising the possibility of drastic developmental changes in the BOLD mechanism (Born et al., 1996; Born et al., 1998; Yamada et al., 1997). Possible reasons for differences in the BOLD signal in children include higher metabolic rates at rest than in adults, perhaps supporting higher synaptic density (Chugani et al., 1988). However, sedation or sleep may instead be the important variable here. Recently, a negative BOLD response was obtained in children and in adults during slow wave sleep, as well as in some cases of sedated adults (Born et al., 2002). Regardless of the precise role of these factors in infants and young children, our own results and those of others suggest that a positive BOLD response dominates in awake children by age 7-8 (Martin et al., 1999).

There are several other concerns that apply to the comparison of children and adults with fMRI, particularly comparison of magnitude measures. Many equipment-related factors affect the exact magnitude of fMRI signals. Furthermore, unlike PET, fMRI does not provide absolute measures of flow or oxygenation; only relative changes can be detected. fMRI signal can be interpreted only with respect to other "baseline" conditions, which is readily accomplished for within-group comparisons (Friston et al., 1996). However, a comparison between groups relies 
on the assumption that both the baseline and the experimental brain states are the same (Bookheimer, 2000).

When subjects perform a task during a scan, controlling for task difficulty becomes a concern. If our groups did not perform the same task during the scanning, then it naturally follows that differences in brain activation could be independent of developmental state. In our case, it is possible that the children differed in fixation stability or extrafoveal spatial attention. However, our fixation task did not involve cognitively difficult reasoning or speeded reactions. To reduce the demands on children with regard to sustained vigilance/attention, we administered the scans in units of $4 \mathrm{~min}$ rather than $8 \mathrm{~min}$. The adult-like retinotopic maps we obtained from the children suggest that they did indeed maintain fixation and attention. Moreover, for the six retinotopic areas, the main effect was the normalized size of several extrastriate areas, and this selective topographic effect is unlikely to be due solely to one of these confounds.

Minimal differences in signal magnitude between children and adults were observed in the ROI analysis, so we can probably exclude major group differences due to head position in the coil or head motion. Once we excluded the subjects with gross head motion artifacts, we observed no correlation between head motion and Fourier magnitude. We did, however, find that the Fourier magnitude measure was variable in children.

To compare the organization of primary visual cortex in more detail, we computed the cortical magnification function in both children and adults. We found that the children's data did not differ from the adults' for V1 or V2. Our values were highly consistent with those reported by previous studies. For example, the exponent fitted to the V1 data of Engel et al. (1997) was 0.063 for two adults, and the data from Sereno et al. (1995) is approximately 0.082. These values are close to our value of 0.074 for 10 adults. Interestingly, the V1 cortical magnification factor has been shown to be correlated with behavioral performance on Vernier acuity tasks (Duncan and Boynton, 2003). Given that Vernier acuity does not reach adult levels until age 14, the adult-like cortical magnification factor we found for the child group is notable. This may be another example in which development of complex sensorimotor behaviors may lag relative to isolated physiological indices (in visual cortex).

We measured the surface area of retinotopic visual areas in terms of absolute and proportional size. Similar data have recently been reported for absolute size for three visual areas in adults only (Dougherty et al., 2003). These authors obtained mean sizes of 1470, 1115, 
and $819 \mathrm{~mm}$ for V1, V2, and V3. The value for V2 is the same as our measurements, although our estimates for V1 and V3 are lower. There are several methodological differences that could provide an explanation: cortical flattening techniques, our use of field sign computations, and our use of manual versus automatic tracing of visual boundaries. Nonetheless, all of our methods were applied consistently to the adults and children, and should allow meaningful comparison between the two groups.

Our data indicate that extrastriate cortex was measurably smaller in children compared to adults. This result was obtained for both absolute size and percentage of the entire reconstructed neocortical sheet, suggesting that gross brain size is not a relevant factor. Consistently, previous literature indicates no significant change in cerebral volume after age 5 (Giedd et al., 1996; Reiss et al., 1996). The idea that extrastriate cortex could mature later than striate cortex is confirmed by the results of some previous reports. Ossenblok et al. (1994) concluded that striate activity dominates the checkerboard onset evoked potential of the children aged about 4-8 years, whereas extrastriate activity grows later in life. A posterior-to-anterior maturation gradient is also suggested by the few available anatomical studies of children's brains (Garey, 1984; Thompson et al., 2000; Sowell et al., 1999). Nonetheless, the fact that our data showed adultlike cortical magnification functions in V2 despite its smaller size in children may indicate that the overall size difference has limited functional significance. Future studies of extrastriate cortical areas in children may help clarify these issues.

We performed whole brain across-subject averaging to assess the regional extent of retinotopic activity in higher level cortex of the parietal, lateral occipital, and temporal cortex. Based on gross anatomical homologies, the eccentricity stimulus results suggest a slight trend toward greater activity in parietal lobes of the adults. However, we cannot easily separate fixation task-related activity from a true difference in retinotopy. Further study of spatial perception and attention in children would be warranted. The most striking difference between children and adults was seen for the polar angle stimulus (Fig. 3-8). However, caution is required when interpreting these data, given that we could not directly compare the hemispheres of adults and children. However, we can still see that within the adult group, the right hemisphere produced more activity, whereas in the child group, the opposite bias was seen. Furthermore, these suggestive differences occur in the absence of differences elsewhere (e.g., ventral temporal cortex). 
There is only a small literature that speaks to hemispheric lateralization during human cortical development, but there is evidence that developmental rates do differ between hemispheres. However, no simple left-right gradient is likely to exist, rather, regionally specific effects have often been found at different ages (Thatcher et al., 1987; Sowell et al., 2001). Given our lateralized findings with the moving polar angle stimulus, one study that measured cortical activity during a form-from-motion task in the left or right hemifield is quite relevant (HollantsGilhuijs et al., 1998). This cross-sectional ERP study of children and adults concluded that "maturation of motion sensitive areas of the extrastriate cortex in children's right hemisphere is delayed with respect to that of the homologous regions of the left hemisphere." The convergence of their conclusion with our own is suggestive, although clearly more studies that measure visual performance along with brain activity are required. Finally, it may be relevant that cognitive tasks such as analysis of global versus local object structure, when measured with fMRI, do not produce the adult-like degree of hemispheric lateralization in many children aged 12-14 years (Moses et al., 2002).

Despite the subtle differences between adults and children we documented here, the overall similarity between the groups is evident. The rather mature visual maps seen in children aged between 9 and 12 years contrast with many cognitive functions that mature much later, and fMRI is increasingly being employed to study such protracted neurological development. For example, the first pediatric fMRI study focused on immature frontal lobe function as assessed by working memory (Casey et al., 1995). Recent cross-sectional studies of reading (Turkeltaub et al., 2003) and visuo-spatial working memory (Kwon et al., 2002) have included extensive behavioral measures and document impressive amounts of both age- and performance-related change. The relatively stable retinotopic visual representations during the childhoodadolescence period may serve as a baseline for comparison to the more protracted development of anterior regions. 


\subsection{Conclusions}

In this study, we measured retinotopic organization in children in multiple visual areas for the first time. We demonstrated the feasibility of applying techniques developed for adults, with only slight modifications. The children were not given separate training sessions, although the reports of other investigators indicate that this is an effective strategy and could improve the success rate even further. In the future, the fMRI technique, especially with longitudinal designs, should contribute greatly to studies of brain development because experiments can be repeated to document change over time. Cortical flattening methods can further facilitate the accumulation of data from multiple experiments onto individual maps of visual cortex. It will be possible to compare retinotopic maps in normal children to children with visual disorders and monitor the effect of treatment variables over time, an approach that is already revealing neurological effects of remediation in children with dyslexia (Temple et al., 2003). 
3.7 Tables

\begin{tabular}{lllllll}
\hline $\begin{array}{l}\text { Visual } \\
\text { Area }\end{array}$ & $\begin{array}{l}\text { Child } \\
\text { LH } \\
\text { polar }\end{array}$ & $\begin{array}{l}\text { Adult } \\
\text { LH } \\
\text { polar }\end{array}$ & $\begin{array}{l}\text { Sig. } p \\
\text { LH } \\
\text { polar }\end{array}$ & $\begin{array}{l}\text { Child } \\
\text { LH } \\
\text { eccen }\end{array}$ & $\begin{array}{l}\text { Adult } \\
\text { LH } \\
\text { eccen }\end{array}$ & $\begin{array}{l}\text { Sig. } p \\
\text { LH } \\
\text { eccen }\end{array}$ \\
\hline V1 & 3.1 & 2.6 & - & 7.6 & 5.4 & - \\
V2 & 3.7 & 3.3 & - & 9.5 & 6.7 & - \\
V3 & 5.7 & 4.7 & - & 12.4 & 9.3 & - \\
V3A & 5.3 & 4.1 & - & 9.3 & 7.2 & - \\
VP & 4.0 & 3.9 & - & 9.8 & 6.4 & - \\
V4V & 3.9 & 4.1 & - & 5.7 & 5.6 & - \\
All & 25.7 & 22.7 & - & 54.3 & 40.6 & - \\
\hline
\end{tabular}

Table 1. Left hemisphere differences between children and adults for polar angle and eccentricity Fourier magnitude across visual areas. 


\begin{tabular}{lllllll}
\hline $\begin{array}{l}\text { Visual } \\
\text { Area }\end{array}$ & $\begin{array}{l}\text { Child } \\
\mathrm{RH} \\
\text { polar }\end{array}$ & $\begin{array}{l}\text { Adult } \\
\mathrm{RH} \\
\text { polar }\end{array}$ & $\begin{array}{l}\text { Sig. } p \\
\mathrm{RH} \\
\text { polar }\end{array}$ & $\begin{array}{l}\text { Child } \\
\mathrm{RH} \\
\text { eccen }\end{array}$ & $\begin{array}{l}\text { Adult } \\
\mathrm{RH} \\
\text { eccen }\end{array}$ & $\begin{array}{l}\text { Sig. } p \\
\mathrm{RH} \\
\text { eccen }\end{array}$ \\
\hline V1 & 2.0 & 3.0 & - & 5.8 & 4.9 & - \\
V2 & 3.1 & 4.0 & - & 8.4 & 7.1 & - \\
V3 & 6.3 & 5.1 & - & 13.0 & 7.6 & - \\
V3A & 4.5 & 3.1 & - & 7.8 & 6.6 & - \\
VP & 3.2 & 5.2 & 0.007 & 8.6 & 8.3 & - \\
V4V & 4.9 & 4.1 & - & 8.9 & 5.8 & - \\
All & 24.0 & 24.5 & - & 52.4 & 40.3 & - \\
\hline
\end{tabular}

Table 2. Right hemisphere differences between children and adults for polar angle and eccentricity Fourier magnitude across visual areas. 


\begin{tabular}{lllllll}
\hline $\begin{array}{l}\text { Visual } \\
\text { Area }\end{array}$ & $\begin{array}{l}\text { Child } \\
\text { LH } \\
\mathrm{mm}^{2}\end{array}$ & $\begin{array}{l}\text { Adult } \\
\text { LH } \\
\mathrm{mm}^{2}\end{array}$ & $\begin{array}{l}\text { Sig. } p \\
\text { LH } \\
\mathrm{mm}^{2}\end{array}$ & $\begin{array}{l}\text { Child } \\
\text { LH } \\
\%\end{array}$ & $\begin{array}{l}\text { Adult } \\
\text { LH } \\
\%\end{array}$ & $\begin{array}{l}\text { Sig. } p \\
\text { LH } \\
\%\end{array}$ \\
\hline V1 & 1102 & 1182 & - & 1.3 & 1.4 & - \\
V2 & 851 & 1194 & 0.02 & 1.0 & 1.4 & 0.02 \\
V3 & 417 & 580 & 0.04 & 0.5 & 0.7 & 0.05 \\
V3A & 513 & 678 & - & 0.6 & 0.8 & - \\
VP & 496 & 678 & - & 0.6 & 0.8 & - \\
V4V & 484 & 738 & 0.01 & 0.6 & 0.9 & 0.02 \\
All & 3865 & 5049 & 0.02 & 4.7 & 5.5 & 0.03 \\
\hline
\end{tabular}

Table 3. Left hemisphere differences between children and adults for visual area size. 


\begin{tabular}{lllllll}
\hline $\begin{array}{l}\text { Visual } \\
\text { Area }\end{array}$ & $\begin{array}{l}\text { Child } \\
\mathrm{RH} \\
\mathrm{mm}^{2}\end{array}$ & $\begin{array}{l}\text { Adult } \\
\mathrm{RH} \\
\mathrm{mm}^{2}\end{array}$ & $\begin{array}{l}\text { Sig. } p \\
\mathrm{RH} \\
\mathrm{mm}^{2}\end{array}$ & $\begin{array}{l}\text { Child } \\
\mathrm{RH} \\
\%\end{array}$ & $\begin{array}{l}\text { Adult } \\
\mathrm{RH} \\
\%\end{array}$ & $\begin{array}{l}\text { Sig. } p \\
\mathrm{RH} \\
\%\end{array}$ \\
\hline V1 & 1219 & 1155 & - & 1.5 & 1.4 & - \\
V2 & 866 & 1161 & 0.02 & 1.1 & 1.4 & 0.04 \\
V3 & 381 & 502 & 0.04 & 0.5 & 0.6 & 0.07 \\
V3A & 549 & 540 & - & 0.7 & 0.6 & - \\
VP & 462 & 667 & 0.003 & 0.6 & 0.8 & 0.02 \\
V4v & 597 & 648 & - & 0.7 & 0.8 & - \\
All & 4073 & 4673 & 0.07 & 5.1 & 5.6 & - \\
\hline
\end{tabular}

Table 4. Right hemisphere differences between children and adults for visual area size. 


\subsection{Figures}
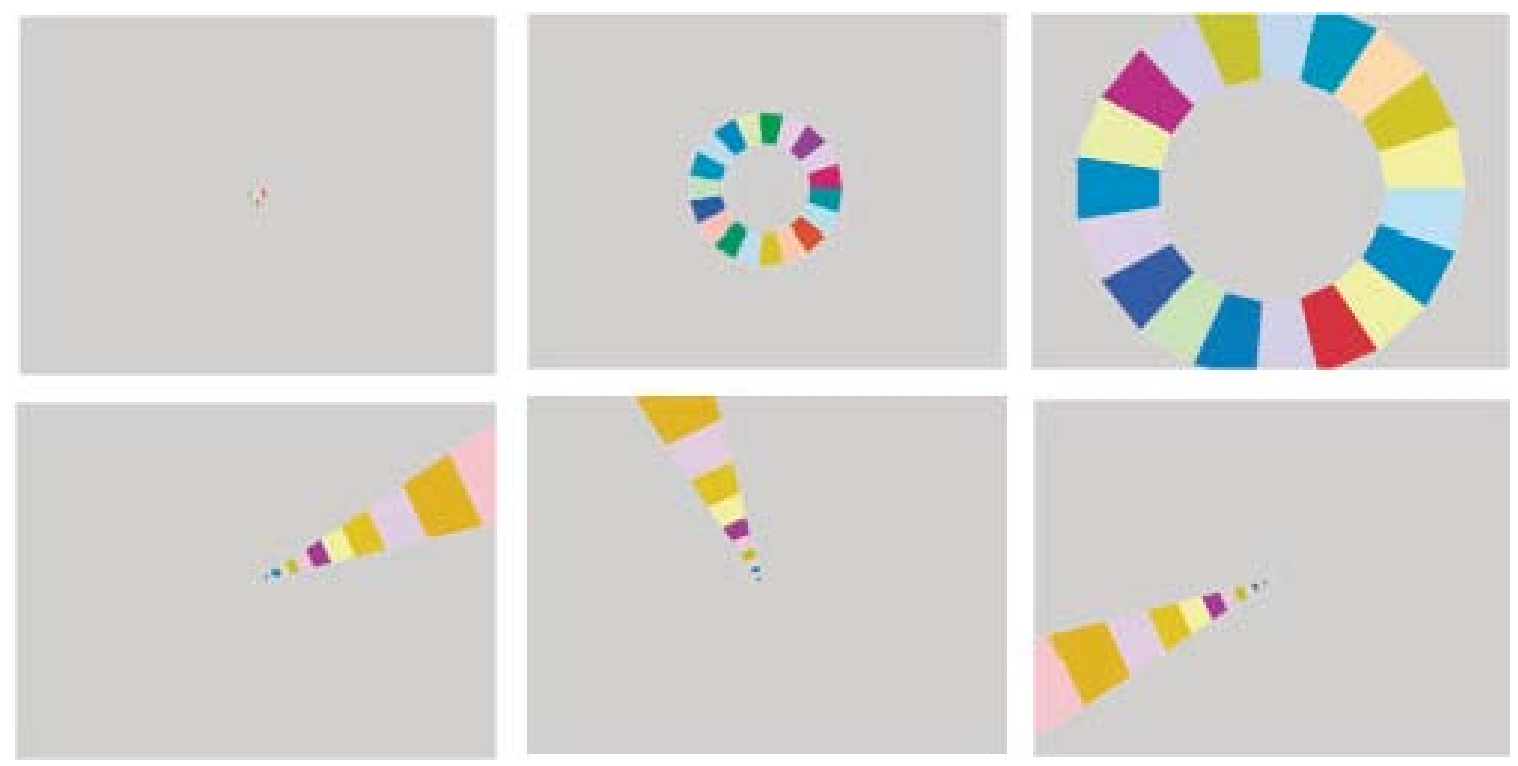

Figure 3-1. Depiction of Dynamic Eccentricity and Polar Angle Stimuli. Top. The eccentricity stimulus was an annular ring that slowly expanded in size. Three isolated example frames are shown. Bottom. The polar angle stimulus was a wedge that rotated around the fixation point. 


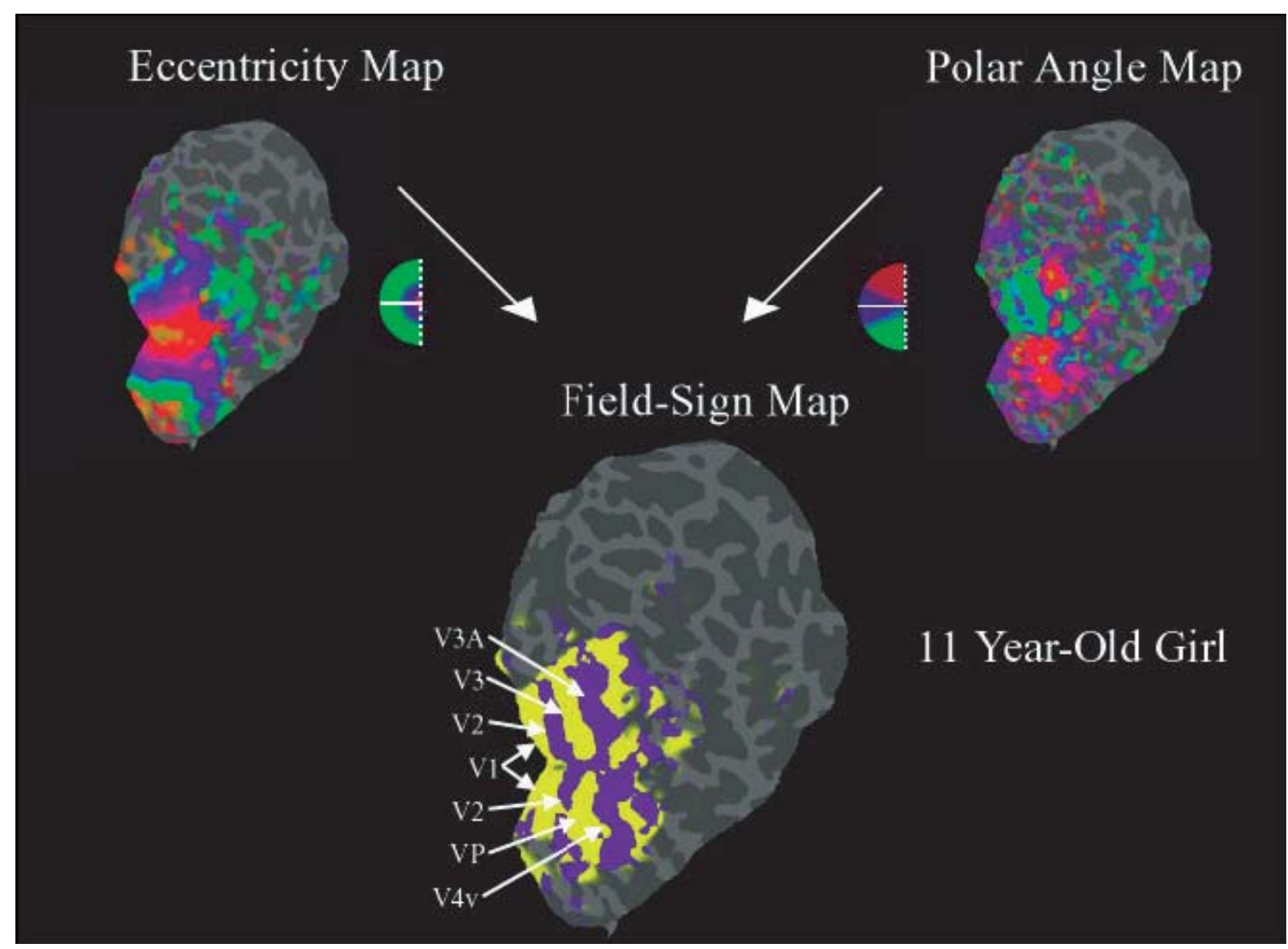

Figure 3-2. Retinotopic Mapping Results from One Child. A. Eccentricity representation is shown on a flattened representation of the right occipital pole. Light and dark grays indicate the unfolded gyri and sulci, respectively. Red, green, and blue indicate the central 1.5-deg, 1.5-5deg, and 5-15-deg eccentricity. The adjacent semicircular logo depicts this color scheme in the corresponding left visual field. B. Polar angle representation is shown in the same subject. As indicated in the adjacent logo, red and green indicate the upper and lower vertical meridians, and blue indicates the horizontal meridians. C. Data sets in A and B are combined to yield the field sign map that indicates the boundaries of multiple visual areas. Yellow indicates areas with quarter or hemifield representation; blue areas have the opposite field sign (i.e., a mirror-reversed map). 


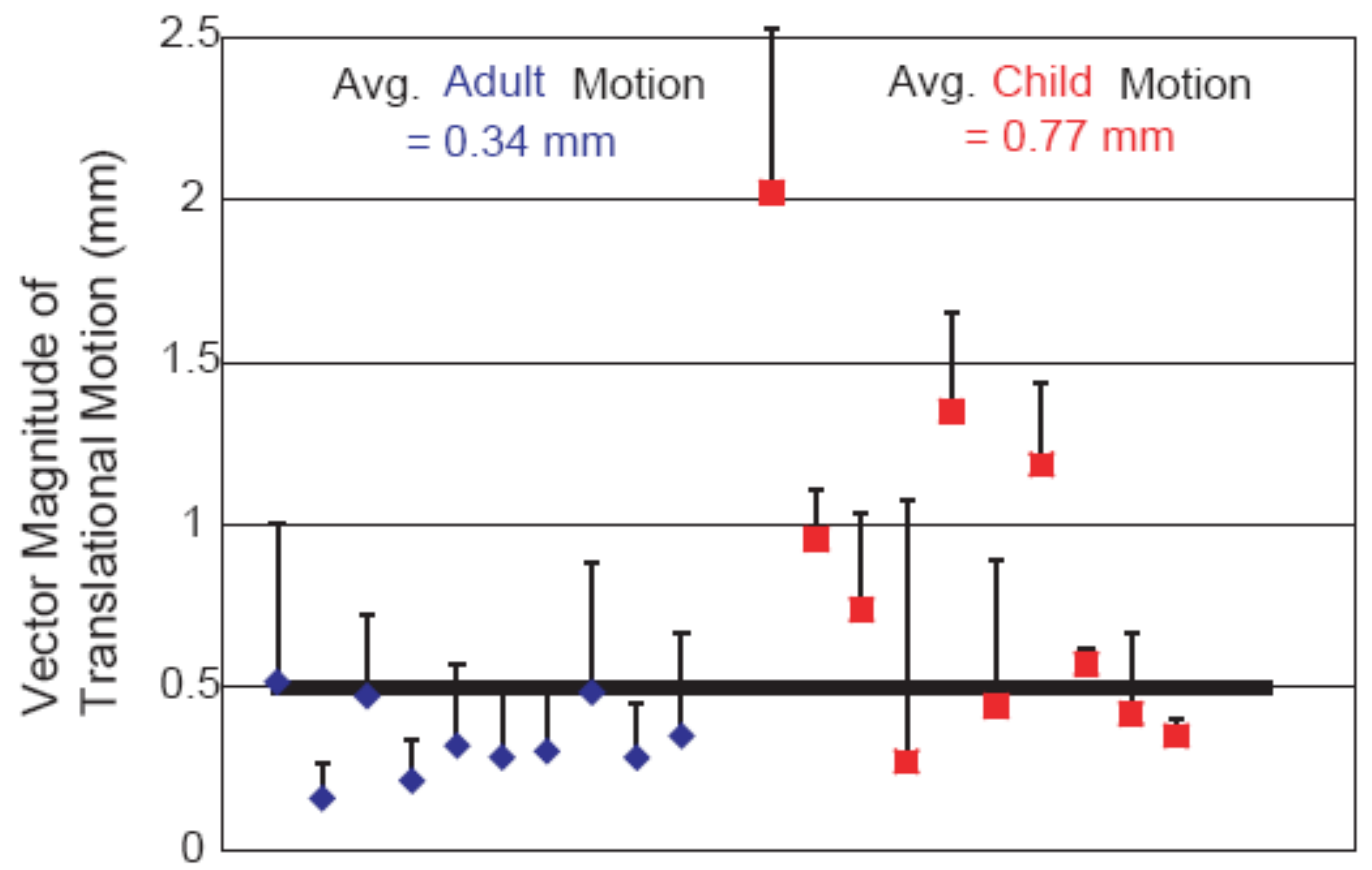

Figure 3-3. COMParison of Average Head Motion During the fMri ScAns for Adults AND CHILDREN. Each data point represents the vector magnitude of translational head motion detected by the motion-correction algorithm. Adults are indicated with blue diamonds, children with red squares. As a population, children produced more movement, but some children were indistinguishable from adults. The thick horizontal black line serves as a reference mark. 


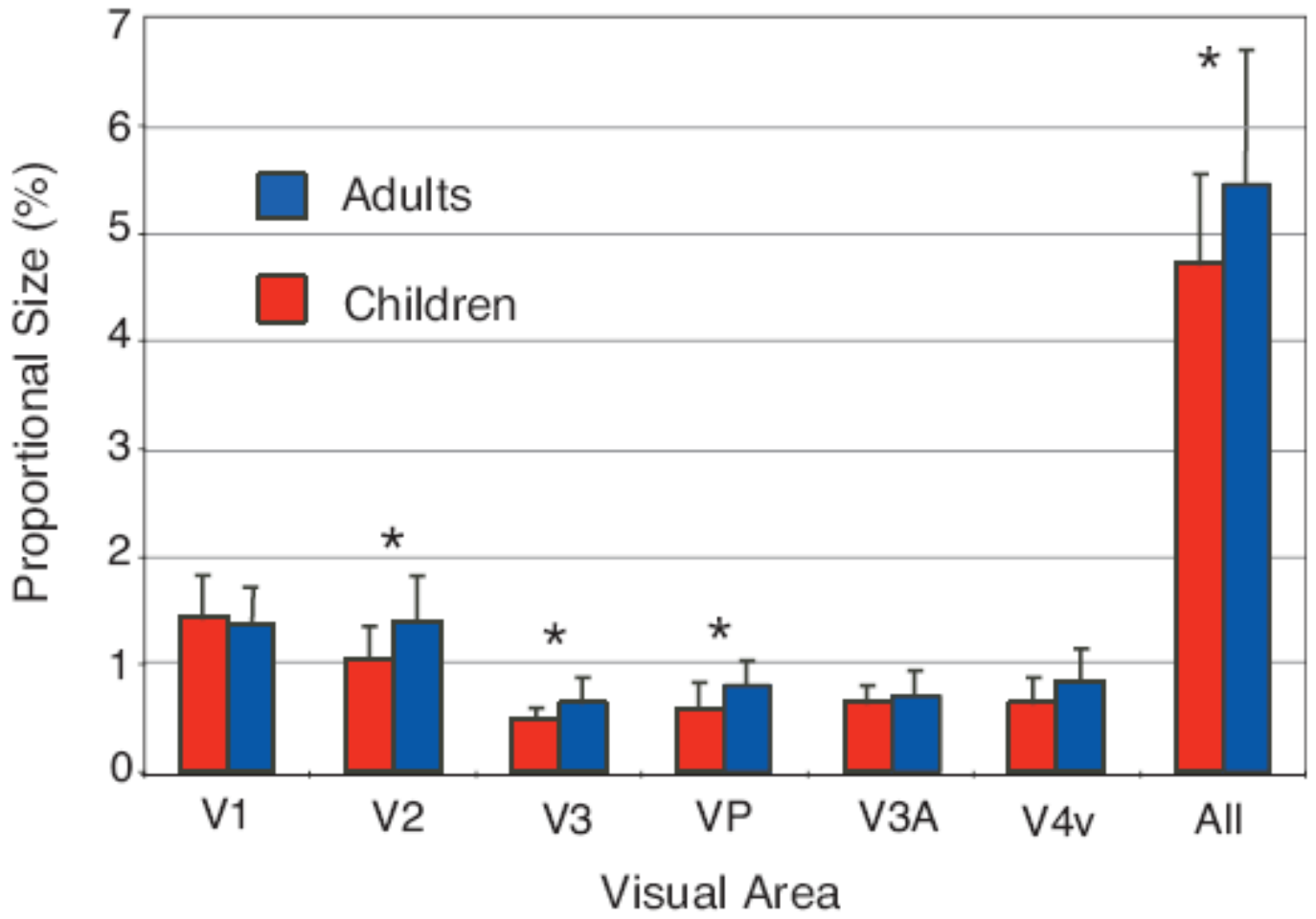

Figure 3-4. COMPARISON OF Size of Visual AREAS In ChILdREN AND Adults. The total combined area (as a percentage of the entire cortical sheet) of homologous visual areas in both hemispheres is plotted. A significant difference between groups is indicated with an asterisk. 


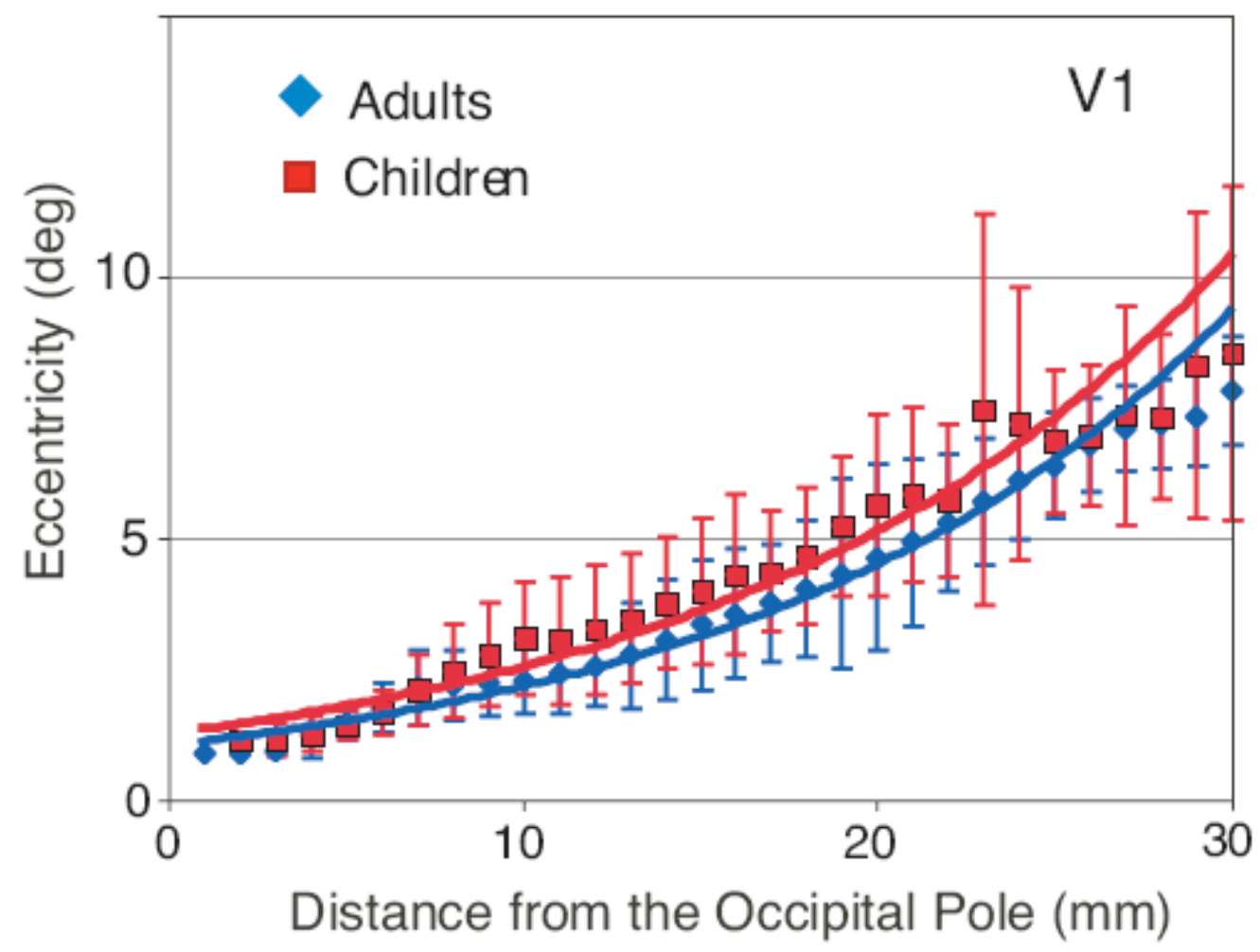

Figure 3-5. COMPARISON OF CORTICAL MAGNifiCATION FunCTIONS IN AREA V1 FOR ChILDREN AND ADULTS. Eccentricity is plotted against cortical distance from the occipital pole along the horizontal meridian. 


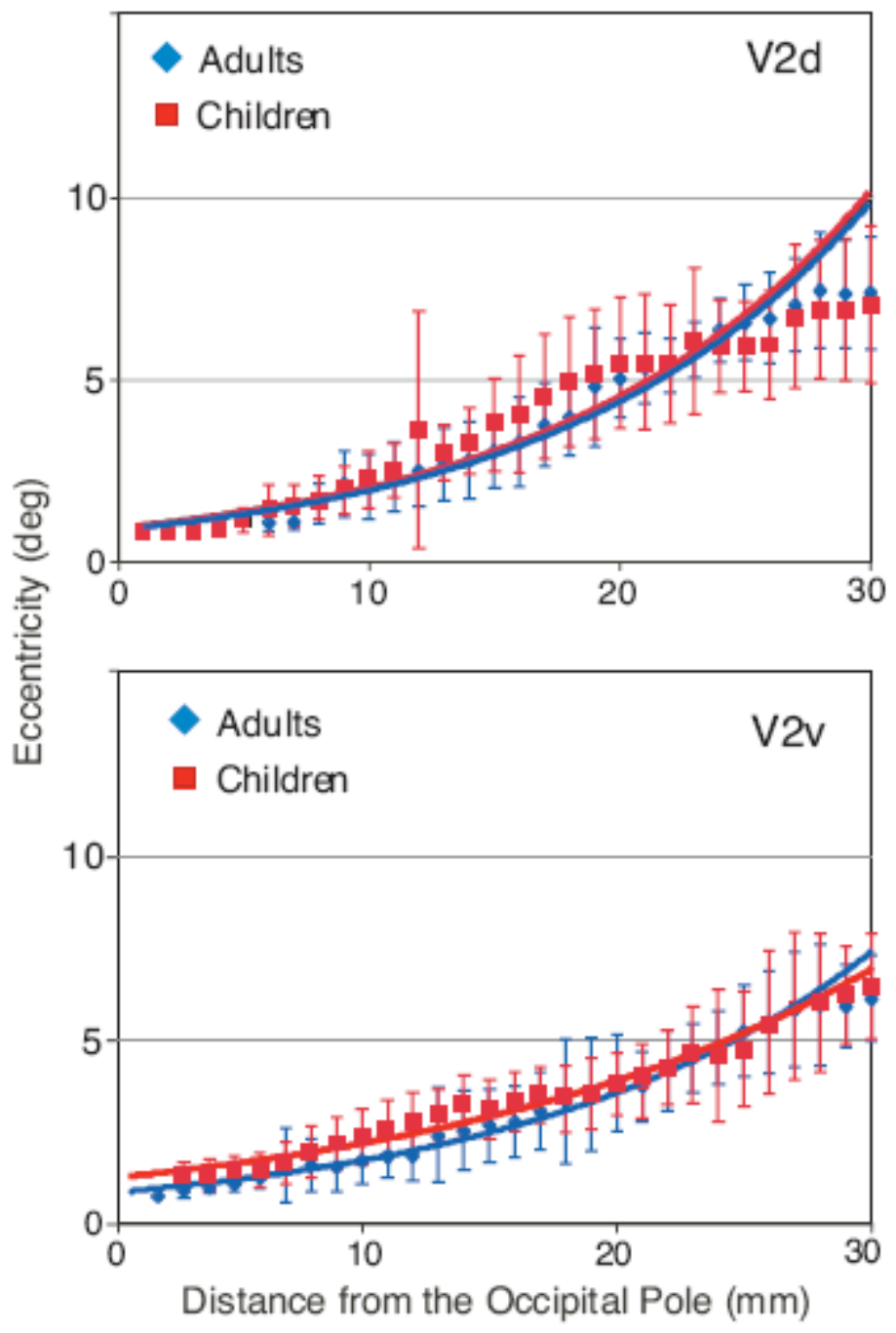

Figure 3-6. COMPARISON OF CORTICAL MAGNifiCATION FunCTIONS IN AREAS V2V AND V2D FOR ChILDREN AND AdUlTS. Eccentricity is plotted against cortical distance from the occipital pole. 


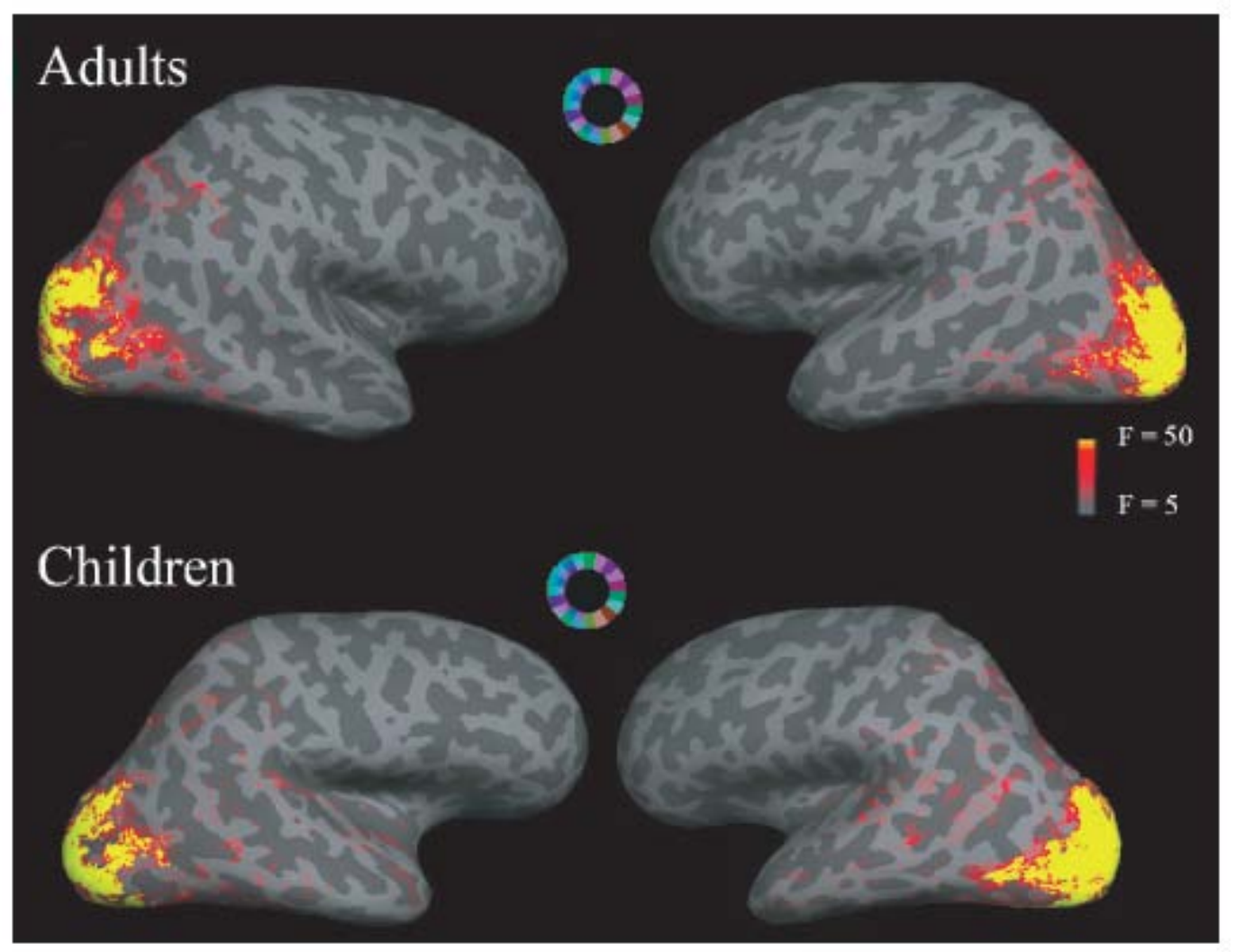

FIGURE 3-7. COMPARISON OF ECCENTRICITY SIGNALS IN LATERAL OCCIPITO-TEMPORAL CORTEX For Adults And Children. Top. The yellow-red color scale shows the Fourier magnitude measured as an F-statistic averaged across 10 adults and plotted on the lateral view of the inflated right and left hemispheres of one representative adult. The central annular symbol indicates the geometry of the eccentricity stimulus. Bottom. The equivalent data are shown for the group of 10 children. The two groups appear qualitatively similar, except for weak signals observed in the parietal cortex of adults that are not visible for the children. 


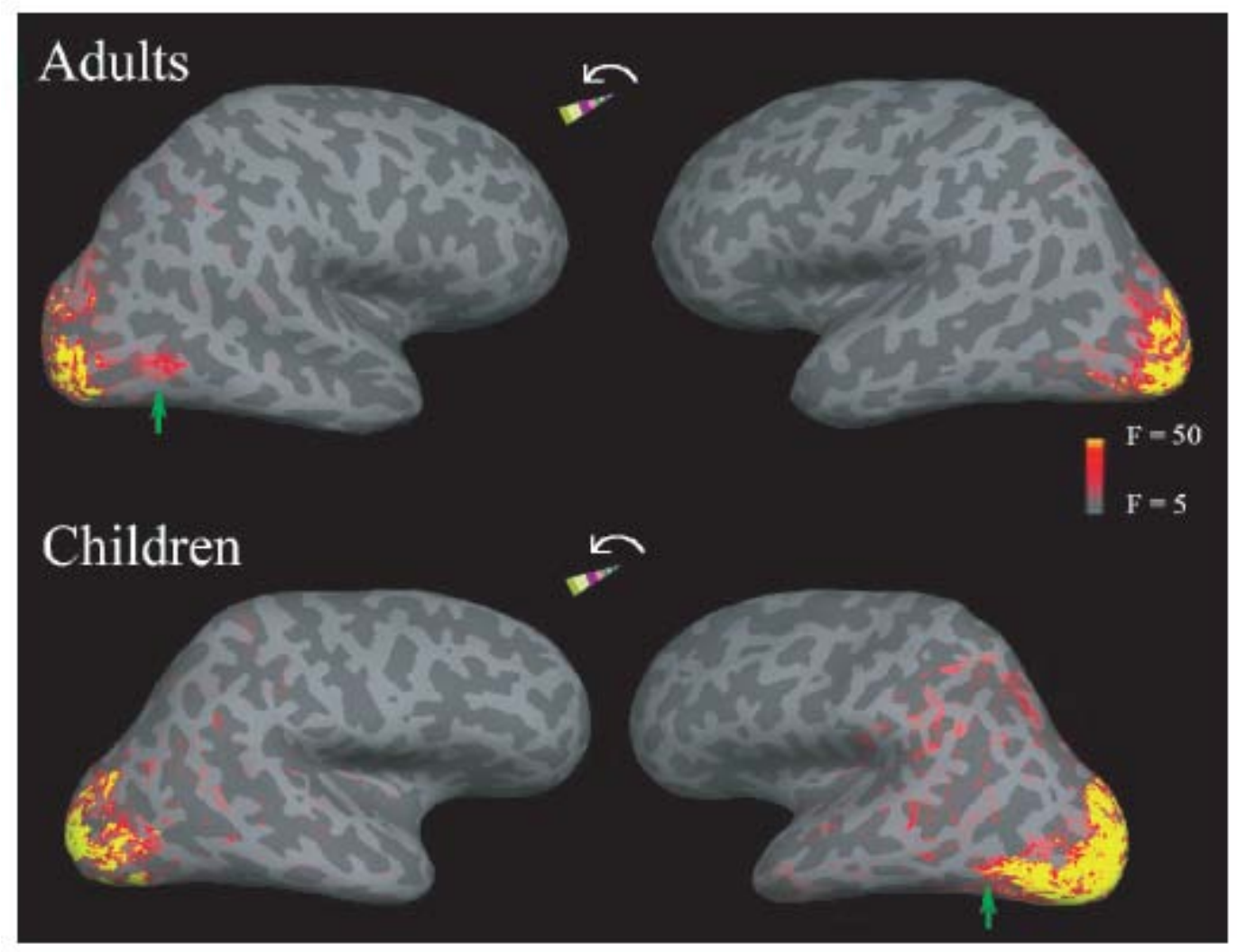

Figure 3-8. Comparison of Polar Angle Signals in Lateral OCCIPITO-Temporal CorteX For Adults And Children. Top. The yellow-red color scale shows the Fourier magnitude measured as an F-statistic averaged across 10 adults and plotted on the lateral view of the inflated right and left hemispheres of one representative adult. The central wedge symbol indicates the geometry of the polar angle stimulus. Bottom. The equivalent data are shown for the group of 10 children. The adults and children differ with respect to which hemisphere shows an advantage in the extent of significant signal (highlighted with green arrows). 


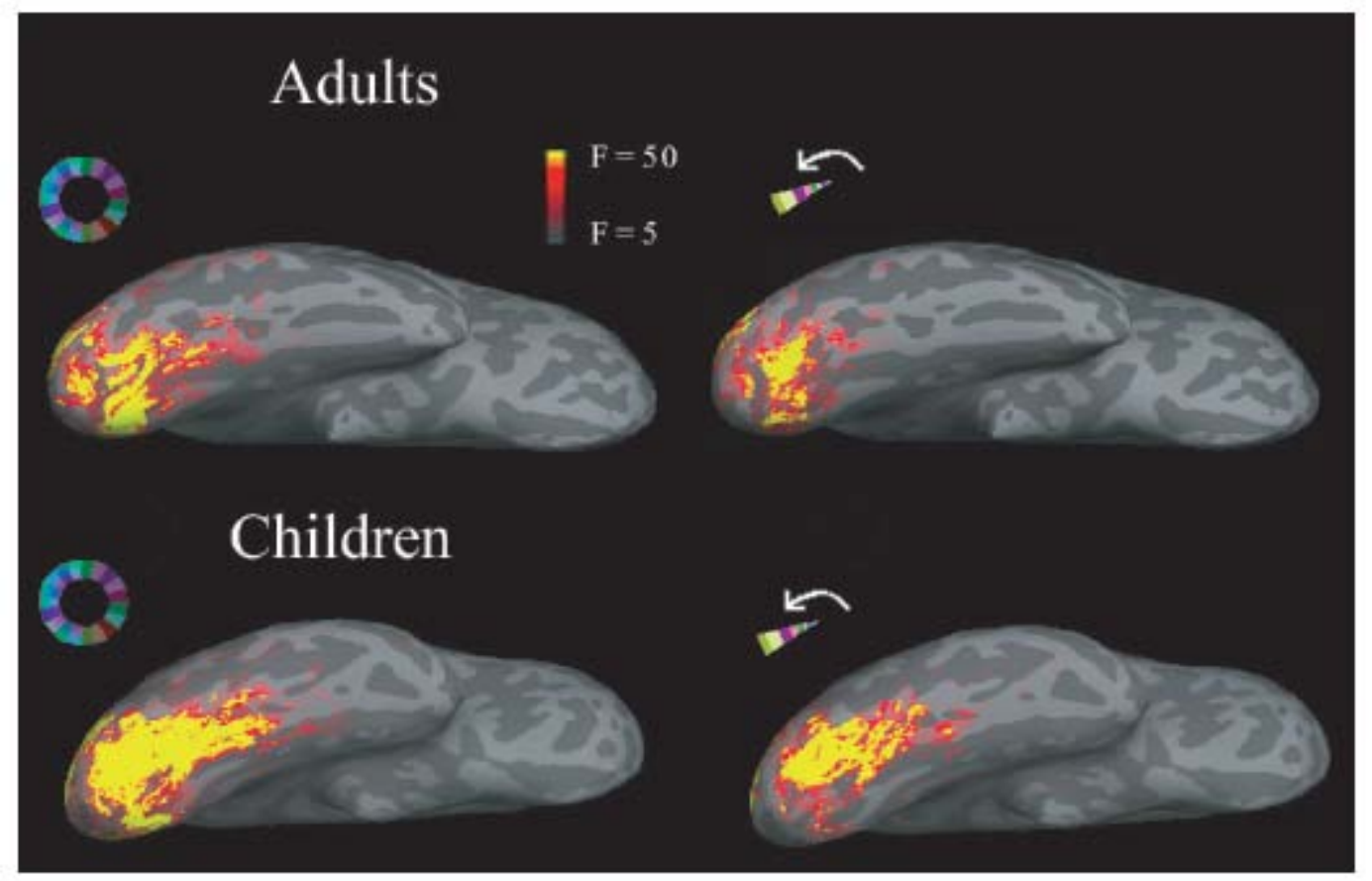

FIgURE 3-9. COMPARISON OF ECCENTRICITY AND POLAR ANGLE SigNALS IN VENTRAL TEMPORAL Cortex for Adults And Children. Top. The yellow-red color scale shows the Fourier magnitude measured as an F-statistic averaged across 10 adults and plotted on the ventral view of the inflated right hemisphere of one representative adult. The annular or wedge symbols indicate when eccentricity or polar angle data are shown. Bottom. The equivalent data are shown for the group of 10 children. The two groups appear qualitatively similar. 


\subsection{References}

Bookheimer SY (2000) Methodological issues in pediatric neuroimaging. Ment Retard Dev Disabil Res Rev 6:161-165.

Born AP, Law I, Lund TE, Rostrup E, Hanson LG, Wildschiodtz G, Lou HC, Paulson OB (2002) Cortical deactivation induced by visual stimulation in human slow-wave sleep. Neuroimage 17:1325-1335.

Born P, Leth H, Miranda MJ, Rostrup E, Stensgaard A, Peitersen B, Larsson HB, Lou HC (1998) Visual activation in infants and young children studied by functional magnetic resonance imaging. Pediatr Res 44:578-583.

Born P, Rostrup E, Leth H, Peitersen B, Lou HC (1996) Change of visually induced cortical activation patterns during development. Lancet 347:543.

Casey BJ, Cohen JD, Jezzard P, Turner R, Noll DC, Trainor RJ, Giedd J, Kaysen D, HertzPannier L, Rapoport JL (1995) Activation of prefrontal cortex in children during a nonspatial working memory task with functional MRI. Neuroimage 2:221-229.

Chugani HT, Shewmon DA, Peacock WJ, Shields WD, Mazziotta JC, Phelps ME (1988) Surgical treatment of intractable neonatal-onset seizures: the role of positron emission tomography. Neurology 38:1178-1188.

Cox RW, Jesmanowicz A (1999) Real-time 3D image registration for functional MRI. Magn Reson Med 42:1014-1018.

Dale AM, Fischl B, Sereno MI (1999) Cortical surface-based analysis. I. Segmentation and surface reconstruction. Neuroimage 9:179-194.

DeYoe EA, Carman GJ, Bandettini P, Glickman S, Wieser J, Cox R, Miller D, Neitz J (1996) Mapping striate and extrastriate visual areas in human cerebral cortex. Proc Natl Acad Sci U S A 93:2382-2386.

Dougherty RF, Koch VM, Brewer AA, Fischer B, Modersitzki J, Wandell BA (2003) Visual field representations and locations of visual areas V1/2/3 in human visual cortex. J Vis 3:586-598.

Duncan RO, Boynton GM (2003) Cortical magnification within human primary visual cortex correlates with acuity thresholds. Neuron 38:659-671.

Ellemberg D, Lewis TL, Liu CH, Maurer D (1999) Development of spatial and temporal vision during childhood. Vision Res 39:2325-2333.

Engel SA, Glover GH, Wandell BA (1997) Retinotopic organization in human visual cortex and the spatial precision of functional MRI. Cereb Cortex 7:181-192. 
Engel SA, Rumelhart DE, Wandell BA, Lee AT, Glover GH, Chichilnisky EJ, Shadlen MN (1994) fMRI of human visual cortex. Nature 369:525.

Evans C, Collins DL, Mills SR, Brown ED, Kelly RL, Peters TM (1993) 3D statistical anatomical models from 305 MRI volumes. pp 1813-1817.

Fischl B, Dale AM (2000) Measuring the thickness of the human cerebral cortex from magnetic resonance images. Proc Natl Acad Sci U S A 97:11050-11055.

Fischl B, Liu A, Dale AM (2001) Automated manifold surgery: constructing geometrically accurate and topologically correct models of the human cerebral cortex. IEEE Trans Med Imaging 20:70-80.

Fischl B, Sereno MI, Dale AM (1999a) Cortical surface-based analysis. II: Inflation, flattening, and a surface-based coordinate system. Neuroimage 9:195-207.

Fischl B, Sereno MI, Tootell RB, Dale AM (1999b) High-resolution intersubject averaging and a coordinate system for the cortical surface. Hum Brain Mapp 8:272-284.

Friston KJ, Price CJ, Fletcher P, Moore C, Frackowiak RS, Dolan RJ (1996) The trouble with cognitive subtraction. Neuroimage 4:97-104.

Garey LJ (1984) Structural development of the visual system of man. Hum Neurobiol 3:75-80.

Giedd JN, Vaituzis AC, Hamburger SD, Lange N, Rajapakse JC, Kaysen D, Vauss YC, Rapoport JL (1996) Quantitative MRI of the temporal lobe, amygdala, and hippocampus in normal human development: ages 4-18 years. J Comp Neurol 366:223-230.

Glover G (1999) Simple analytic spiral K-space algorithm. Magn Reson Med 42:412-415.

Hadjikhani N, Liu AK, Dale AM, Cavanagh P, Tootell RB (1998) Retinotopy and color sensitivity in human visual cortical area V8. Nat Neurosci 1:235-241.

Hasson U, Levy I, Behrmann M, Hendler T, Malach R (2002) Eccentricity bias as an organizing principle for human high-order object areas. Neuron 34:479-490.

Hollants-Gilhuijs MA, Ruijter JM, Spekreijse H (1998) Visual half-field development in children: detection of motion-defined forms. Vision Res 38:651-657.

Kiorpes L, Movshon JA (2003) Neural limitations on visual development in primates. In: The visual neurosciences (Chalupa L, Werner JS, eds), pp 159-173. Cambridge, MA: MIT Press.

Kovacs I (2000) Human development of perceptual organization. Vision Res 40:1301-1310.

Kovacs I, Kozma P, Feher A, Benedek G (1999) Late maturation of visual spatial integration in humans. Proc Natl Acad Sci U S A 96:12204-12209.

Kwon H, Reiss AL, Menon V (2002) Neural basis of protracted developmental changes in visuospatial working memory. Proc Natl Acad Sci U S A 99:13336-13341. 
Kwong KK, Belliveau JW, Chester DA, Goldberg IE, Weiskoff RM, Poncelet BP, Kennedy DN, Hoppel BE, Cohen MS, Turner R, Rosen B, Brady TJ (1992) Dynamic magnetic resonce imaging of human brain activity during primary sensory stimulation. Proc Natl Acad Sci U S A 89:5675-5679.

Logothetis NK, Pauls J, Augath M, Trinath T, Oeltermann A (2001) Neurophysiological investigation of the basis of the fMRI signal. Nature 412:150-157.

Malach R, Levy I, Hasson U (2002) The topography of high-order human object areas. Trends Cogn Sci 6:176-184.

Martin E, Joeri P, Loenneker T, Ekatodramis D, Vitacco D, Hennig J, Marcar VL (1999) Visual processing in infants and children studied using functional MRI. Pediatr Res 46:135-140.

Mendola JD, Dale AM, Fischl B, Liu AK, Tootell RB (1999) The representation of illusory and real contours in human cortical visual areas revealed by functional magnetic resonance imaging. J Neurosci 19:8560-8572.

Moses P, Roe K, Buxton RB, Wong EC, Frank LR, Stiles J (2002) Functional MRI of global and local processing in children. Neuroimage 16:415-424.

Ogawa S, Tank W, Menon RS, Ellermann JM, Kim SG, Merkle H, Ugurbil K (1992) Intrinsic signal changes accompanying sensory stimulation: functional brain mapping with magnetic resonce imaging. Proc Natl Acad Sci U S A 89:5951-5955.

Ossenblok P, de Munck JC, Wieringa HJ, Reits D, Spekreijse H (1994) Hemispheric asymmetry in the maturation of the extrastriate checkerboard onset evoked potential. Vision Res 34:581590.

Regan D, Spekreijse H (1986) Evoked potentials in vision research 1961-86. Vision Res 26:1461-1480.

Reiss AL, Abrams MT, Singer HS, Ross JL, Denckla MB (1996) Brain development, gender and IQ in children. A volumetric imaging study. Brain 119 ( Pt 5):1763-1774.

Rosas HD, Liu AK, Hersch S, Glessner M, Ferrante RJ, Salat DH, van Der KA, Jenkins BG, Dale AM, Fischl B (2002) Regional and progressive thinning of the cortical ribbon in Huntington's disease. Neurology 58:695-701.

Sereno MI, Dale AM, Reppas JB, Kwong KK, Belliveau JW, Brady TJ, Rosen BR, Tootell RB (1995) Borders of multiple visual areas in humans revealed by functional magnetic resonance imaging. Science 268:889-893.

Sireteanu R (2000) Texture segmentation, "pop-out," and feature binding in infants and children. In: Progress in infancy research (Rovee-Collier C, Lipsitt L, Hayne H, eds), pp 183-250. Mahwah, NJ: Lawrence Erlbaum. 
Skoczenski AM, Norcia AM (2002) Late maturation of visual hyperacuity. Psychol Sci 13:537541.

Sowell ER, Thompson PM, Holmes CJ, Batth R, Jernigan TL, Toga AW (1999) Localizing agerelated changes in brain structure between childhood and adolescence using statistical parametric mapping. Neuroimage 9:587-597.

Sowell ER, Thompson PM, Tessner KD, Toga AW (2001) Mapping continued brain growth and gray matter density reduction in dorsal frontal cortex: Inverse relationships during postadolescent brain maturation. J Neurosci 21:8819-8829.

Talairach J, Tournoux P (1988) Co-planar stereotaxic atlas of the human brain. New York: Thieme.

Temple E, Deutsch GK, Poldrack RA, Miller SL, Tallal P, Merzenich MM, Gabrieli JD (2003) Neural deficits in children with dyslexia ameliorated by behavioral remediation: evidence from functional MRI. Proc Natl Acad Sci U S A 100:2860-2865.

Thatcher RW, Walker RA, Giudice S (1987) Human cerebral hemispheres develop at different rates and ages. Science 236:1110-1113.

Thompson PM, Giedd JN, Woods RP, MacDonald D, Evans AC, Toga AW (2000) Growth patterns in the developing brain detected by using continuum mechanical tensor maps. Nature 404:190-193.

Tootell RB, Hadjikhani N, Hall EK, Marrett S, Vanduffel W, Vaughan JT, Dale AM (1998a) The retinotopy of visual spatial attention. Neuron 21:1409-1422.

Tootell RB, Mendola JD, Hadjikhani NK, Ledden PJ, Liu AK, Reppas JB, Sereno MI, Dale AM (1997) Functional analysis of V3A and related areas in human visual cortex. J Neurosci 17:7060-7078.

Tootell RB, Mendola JD, Hadjikhani NK, Liu AK, Dale AM (1998b) The representation of the ipsilateral visual field in human cerebral cortex. Proc Natl Acad Sci U S A 95:818-824.

Turkeltaub PE, Gareau L, Flowers DL, Zeffiro TA, Eden GF (2003) Development of neural mechanisms for reading. Nat Neurosci 6:767-773.

Tyschen L (1992) Binocular vision. In: Adler's physiology of the eye: clinical application (Hart WM, ed), pp 773-853. St. Louis, MO: Mosby.

Wade AR, Brewer AA, Rieger JW, Wandell BA (2002) Functional measurements of human ventral occipital cortex: retinotopy and colour. Philos Trans R Soc Lond B Biol Sci 357:963973.

Yamada H, Sadato N, Konishi Y, Kimura K, Tanaka M, Yonekura Y, Ishii Y (1997) A rapid brain metabolic change in infants detected by fMRI. Neuroreport 8:3775-3778. 
CHAPTER 4: Monocular Representation in Visual Cortex of Amblyopic Adults Measured with fMRI 


\subsection{Abstract}

Amblyopia is a developmental disorder that results in abnormalities in primary visual cortex, although the extent of further cortical involvement remains unclear. This fMRI study directly compared the BOLD signals produced by monocular stimulation in 18 adults (6 esotropic strabismics, 6 anisometropes, and 6 controls). Measures were made in three cortical regions-ofinterest (ROIs), individually defined using standard retinotopic mapping techniques in the nonamblyopic eye, corresponding to extrafoveal V1, extrafoveal V2, and the foveal representation at the occipital pole. Fixation stability was continuously monitored and found not to differ significantly between subject groups. Overall, results showed depressed fMRI signal magnitude for amblyopic eyes compared with fellow eyes in all ROIs, although a few subjects did not show this trend. Assessment of the spatial extent of activation using an ocular dominance index revealed significantly larger interocular differences for both strabismics and anisometropes compared with controls. In addition, both amblyopic groups showed fewer vertices able to be driven by both eyes. Analysis of individual phase-encoded retinotopic maps obtained from amblyopic eyes revealed fewer vertices in the occipital pole activated by foveal stimuli, consistent with known psychophysical losses. Finally, several amblyopic subjects demonstrated the unexpected finding of increased activity in parietal and temporal cortex for the amblyopic eye. We conclude that, even in a heterogeneous population, abnormal early visual experience commonly leads to regionally specific cortical adaptations. 


\subsection{Introduction}

Amblyopia, often referred to as "lazy eye," is the most common visual disorder in children (Hillis et al., 1983). It is defined as visual impairment without ocular lesion, resulting instead from abnormal neural development due to atypical early visual experience (Campos, 1995). Visual acuity is usually unimpaired in the nonamblyopic eye.

The two most prevalent amblyogenic factors are unequal interocular refractive error and ocular deviation, sometimes presenting concurrently (von Noorden and Campos, 2001). Unbalanced refractive error results in anisometropic amblyopia, whereas strabismic amblyopia follows ocular deviation. Less frequently, unilateral cataract can cause deprivation amblyopia (Barrett et al., 2004). The common factor in amblyopia, regardless of etiology, is interruption of normal visual experience during critical developmental periods (Daw, 1998). Consequently, studies of amblyopic adults afford an opportunity to understand the effects of early sensory impairment on subsequent brain organization and function.

Earlier investigations of human amblyopia relied upon careful psychophysical testing, finding consistently impaired visual acuity and contrast sensitivity in amblyopic eyes (Abrahamsson and Sjostrand, 1988; Asper et al., 2000b; Ciuffreda and Fisher, 1987), particularly in central vision (Thomas, 1978; Sireteanu and Fronius, 1990). Furthermore, asymmetric visual field deficits have been reported in amblyopes with esotropic (i.e., inward deviating) strabismus, with nasal impaired more than temporal retina input (Sireteanu and Fronius, 1981; Hess and Pointer, 1985). The amblyopic deficit is thought to be based in primary visual cortex (V1), where monocular inputs are first combined (Cynader, 1982; Hess, 2001; Asper et al., 2000a). Animal deprivation models utilizing eyelid suture show grossly normal retinas and lateral geniculate nuclei, but dramatic shifts in ocular dominance in V1 layer IV (Wiesel and Hubel, 1963; Hubel et al., 1976). Accordingly, few neurons respond to the sutured eye, and binocularity is severely impaired. Subsequent animal studies utilizing models of human anisometropic (Movshon et al., 1987) and strabismic (Kiorpes and Boothe, 1981) amblyopia show milder

ocular dominance shifts, less severe binocularity losses, and impaired cortical acuity and contrast sensitivity for the affected eye (Crawford and Harwerth, 2004; Smith, III et al., 1997). These data match the human psychophysics well, suggesting similar neural substrates. 
The neuroimaging techniques of positron-emission tomography (PET) and functional magnetic resonance imaging (fMRI) have recently expanded the study of human amblyopia. PET has shown reduced cerebral blood flow and glucose metabolism in V1 and extrastriate cortex for amblyopic eye viewing (Mizoguchi et al., 2005; Demer et al., 1997; Imamura et al., 1997), and fMRI has been used to quantify reduced activation extent (Goodyear et al., 2000; Algaze et al., 2002) and signal magnitude in occipital cortex (Barnes et al., 2001; Liu et al., 2004). Furthermore, a few studies have also compared amblyopic subtypes, finding less activation at high spatial frequencies for anisometropes (Choi et al., 2001), but more impaired binocular responses in strabismics (Lee et al., 2001). Most studies have not localized deficits to specific field locations or visual areas.

The present study compared strabismic and anisometropic amblyopes with matched controls. First, we administered psychophysical tests to confirm diagnoses. We used monocular hemifield mapping to examine interocular and between-group metabolic signal differences. We then performed retinotopic mapping, and defined V1, V2, and a foveal region-of-interest. We addressed four hypotheses: 1.) Amblyopic eyes will produce reduced signal magnitude. 2.) Ocular dominance will shift toward the fellow eye and binocularity will be impaired. 3.) The nasal retina will be more impaired in strabismic amblyopes. 4.) Early visual areas will have abnormal representations of central visual space. We found reduced amblyopic eye signal, shifted ocular dominance, and impaired binocularity in amblyopic subjects. Moreover, we found visual field remapping in occipital pole locations that represented foveal space in the fellow eye, but parafoveal locations in the amblyopic eye. Unexpectedly, we also found abnormal activation patterns in parietal and temporal cortex of three amblyopes, a potential signature of higher-order deficits. 


\subsection{Materials and Methods}

\subsubsection{Subjects}

We studied 18 adult volunteers aged 18 to 35 (13 female, 5 male). Six were control subjects (CTL), six had previously been diagnosed with strabismic amblyopia (STRAB), and six had previously been diagnosed with anisometropic amblyopia (ANISO). Any subject with other known or suspected neurological or psychiatric conditions was excluded. These subjects were recruited through public advertisement in the surrounding regions of West Virginia, Pennsylvania, and Maryland. Informed consent was obtained from all subjects (IRB protocol \# 14788), in accordance with the Code of Ethics of the World Medical Association (Declaration of Helsinki).

Our subject groups were matched for mean age $(\mathrm{CTL}=25, \mathrm{STRAB}=27, \mathrm{ANISO}=28)$ and mean years of education $(\mathrm{CTL}=15, \mathrm{STRAB}=16, \mathrm{ANISO}=15)$. All amblyopic subjects had a history of patch occlusion treatment during childhood, but the presence of visual impairment at the time of testing demonstrates that the deficit was never completely reversed (Table 1). Three of the six strabismic subjects also reported surgical correction of their deviation in childhood.

All subjects completed a full ophthalmologic exam at the WVU Eye Institute to confirm their diagnosis. Diagnosis of anisometropic amblyopia was assigned on the basis of 1.) Interocular refractive difference of hyperopia $>=+1.0$ diopter, astigmatism $>=+1.0$ diopter, or myopia $>=-2.5$ diopters; or 2.) History of anisometropia but no history of strabismus or strabismus surgery. Diagnosis of strabismus was made on the basis of a history of strabismus or strabismus surgery, but no anisometropia (as defined above). In clinical practice it is common to find that some subjects with amblyopia present with a mixed anisometropic/strabismic diagnosis, although little consensus exists regarding additional subtypes. One of our strabismic subjects (S4) had an interocular difference of hyperopia of 2.75 diopters, consistent with a potential mixed diagnosis.

The direction and magnitude of strabismic deviation in our subjects was determined with cover-uncover, alternate cover, and prism testing. All six of our strabismic subjects showed inward eye deviations (esotropia) rather than outward deviations (exotropia). Congenital esotropia and exotropia are commonly associated with amblyopia, although it has previously 
been shown that esotropia has the greater prevalence in Caucasian populations (Ing and Pang, 1974). Latent deviations for our subjects ranged from 0-25 prism diopters (Table 1).

The ophthalmologic tests also included examination of the fundus with dilation, documentation of ductions and versions, autorefraction, and a sensory exam including Snellen visual acuity (Lombart Instrument, Norfolk, VA), contrast sensitivity (Lighthouse International, New York, NY), Worth 4-dot, stereoacuity (Titmus Optical, Inc., Petersburg, VA), and Ishihara color plates (Kanehara and Co., Ltd., Tokyo, Japan). The results for Snellen visual acuity, interocular refractive difference, and the Titmus stereoacuity are given in Table 1. The Titmus stereoacuity test was scored according to highest level of detectable horizontal disparity for the Wirt rings or for the Titmus fly. The crudest stereoacuity measurable with this test is 3500 , assigned for patients able to perceive disparity only in the Titmus fly illustration.

Finally, in order to facilitate comparison of amblyopic and control subjects, individuals in the control group were assigned a dominant eye (DE) and a non-dominant eye (NDE) based on visual acuity. We used Snellen acuity was the primary criterion, but in three subjects grating acuity was used because Snellen acuity was equal in both eyes. All amblyopic subjects had worse Snellen and grating acuity in their amblyopic eye (AE) than their fellow eye (FE). In all subsequent group analyses, AE was always compared with NDE, and FE with DE.

\subsubsection{Psychophysical Testing}

All psychophysical tests were administered with optical correction. Translucent plastic patches covered the non-testing eye. All tests included practice trials to ensure that the stimuli were visible and the task instructions were adequately understood.

\subsubsection{Grating Acuity}

Stimuli were generated using the Psychophysics Toolbox (Brainard, 1997; Pelli, 1997) and MATLAB 5 for Macintosh OS 9 and were displayed on a calibrated high resolution CRT monitor (Hitachi Super Scan 812) at 5.9 meters. For each trial, the subject viewed two temporally sequenced screens identified by audible tone, containing either a vertical sinusoidal grating (80\% physical contrast, subtending 8 degrees of visual angle) or an isoluminant gray screen. One-third of trials were blanks. When present, grating contrast was ramped-on over 200 
msec, plateaued for $500 \mathrm{msec}$, and then ramped-off over $200 \mathrm{msec}$. The subject identified the grating epoch using a two-alternative forced choice paradigm (2-AFC). Spatial frequency was varied by a staircase procedure that increased spatial frequency following two correct responses and decreased spatial frequency after one incorrect response (2-up, 1-down), terminating after seven reversals. The acuity threshold was taken as the geometric mean of the last four reversals.

\subsubsection{Contrast Sensitivity}

These stimuli were generated as described above, and displayed on a calibrated Apple Studio Display LCD monitor at $57 \mathrm{~cm}$. For each trial, the subject viewed two temporally sequenced epochs identified by audible tone, containing either a vertical sinusoidal grating (subtending 6 degrees of visual angle, $200 \mathrm{~ms}$ duration) or an isoluminant gray screen. The entire trial lasted $2400 \mathrm{~ms}$ with an intertrial interval of $1800 \mathrm{~ms}$. The subject identified the grating epoch, using 2-AFC. An interactive staircase procedure (2-up, 1-down) was used to approach the contrast detection threshold (the $71 \%$ correct level) at each of five spatial frequencies $(0.5,1.0,2.0,4.0$, and 8.0 cycles per degree), terminating after seven reversals for each spatial frequency. The first three reversals used a step size of $6 \mathrm{~dB}$ to rapidly approach threshold, while the last four reversals used a $2 \mathrm{~dB}$ step size. The contrast threshold was taken as the geometric mean of the last four reversals.

\subsubsection{Contour Integration}

Stimuli were displayed on a Silicon Graphics CRT monitor (model GDM-5411). The task was to determine the orientation (i.e., pointing left or right) of a perceived egg-shaped contour made up of 15 small aligned Gabor patches embedded in a field of randomly oriented patches of identical contrast and spatial frequency (Braun, 1999; Kovacs and Julesz, 1993; Chandna et al., 2001; Field et al., 1993; Pennefather et al., 1999; Kovacs et al., 1999). This test was administered using the method of constant stimuli and a 2-AFC paradigm at six levels of increasing difficulty (available online at http://zeus.rutgers.edu/ ikovacs/S\&P contour.html). Task difficulty increased as a function of increasing orientation jitter of the Gabor patches along the contour. 


\subsubsection{Cortical Surface Reconstruction}

Surface reconstructions of each subject's cerebral cortex were generated from highresolution anatomical images obtained in a General Electric 1.5 Tesla scan session prior to the fMRI experiments, using the freely available (http://www.nmr.mgh.harvard.edu/freesurfer) FreeSurfer software package (Dale et al., 1999; Dale and Sereno, 1993; Fischl et al., 1999; Fischl et al., 2001). Briefly, whole-head 3D Fast Spoiled Grass (FSPGR) gradient echo scans, optimized for contrast between gray and white matter, were collected for each subject. Specific parameters were: fast IR prep (prep time $=300 \mathrm{~ms}$ ), TE $=1.9 \mathrm{~ms}$, flip angle $=20 \mathrm{deg}, \mathrm{FOV}=24$ $\mathrm{cm}$, axial slices, $256 \times 256$ matrix, voxel resolution $=0.94 \times 0.94 \times 1.2 \mathrm{~mm}$.

The cortical surface representation was inflated and then flattened by introducing a series of cuts to the 3-D surface to isolate the occipital pole (Sereno et al., 1995). Using this technique, the primary relaxation cut corresponds to calcarine sulcus, approximately the horizontal meridian representation in V1. The resulting occipital patch was used for fMRI data display, but all data analysis was performed in native space.

\subsubsection{Functional Magnetic Resonance Imaging}

\subsubsection{General Methods}

Subjects were scanned in both a General Electric 3 Tesla MR scanner (Experiment 1) and a General Electric 1.5 Tesla MR scanner (Experiment 2), using a visual surface coil (Nova Medical, Inc., Wilmington, MA), as has been previously described (Mendola et al., 1999; Conner et al., 2004). After a sagittal localizing scan, a T1-weighted inversion recovery sequence $(\mathrm{TR}=$ $400 \mathrm{~ms}$ ) was used to acquire 20 interleaved oblique $4 \mathrm{~mm}$ slices with 0.86 x $0.86 \mathrm{~mm}$ in-plane resolution, oriented perpendicular to the calcarine sulcus, beginning at the occipital pole. These anatomical scans were later utilized to register functional data to the cortical surface model.

The next step was to acquire multiple functional scanning runs using the same slice prescription selected in the anatomical scans, but with $1.72 \times 1.72 \mathrm{~mm}$ (Exp. 1) or $3.44 \times 3.44$ mm (Exp. 2) in-plane resolution. Functional signals reflecting the blood oxygenation level dependent (BOLD) contrast were acquired using a spiral gradient echo sequence [TE $=40 \mathrm{~ms}$, Flip Angle $=65 \mathrm{deg}, \mathrm{TR}=2000 \mathrm{~ms}$ (Exp.1) or TR = $4000 \mathrm{~ms}$ (Exp. 2)] (Kwong et al., 1992; Ogawa et al., 1992; Glover, 1999). 
Head movement (within- and between- scans) was minimized by the use of foam packing and adhesive tape around the subject's head within the MR scanner. Eye movements were monitored using the Sensomotorics iView system in order to ensure fixation stability during the functional scans. The iView system was used to measure gaze position in the stimulated eye, and was calibrated using a nine-point display at a screen resolution of $832 \times 624$ pixels, subtending approximately $30 \mathrm{deg}$ horizontal $\times 23 \mathrm{deg}$ vertical of visual angle. Accurate calibration was not achieved in a minority of subjects (see Results), but most subjects were able to have eye tracking throughout both experiments. The recording rate of the iView camera was $60 \mathrm{~Hz}$.

During the MR imaging experiments, the visual stimuli were generated using the Psychophysics Toolbox and MATLAB 5 for Macintosh OS 9 on a PowerMac G4 computer with dual SVGA display drivers (output resolution $=832 \times 624$ pixels, 30 deg horizontal x 23 deg vertical). The stimuli were displayed in the scanner using the Avotec SilentVision dichoptic projector. Subjects viewed the images with both eyes open by looking straight ahead into the eyepieces, which were placed approximately $1 \mathrm{~cm}$ in front of their eyes. Subjects used the eyepieces' built-in optical correction. For each functional scanning run, one eyepiece displayed the stimulus and fixation target while the other displayed an isoluminant gray screen. Left and right eye stimulation was alternated during each experiment.

A central fixation mark was present at all times for the fMRI experiments. Subjects were clearly instructed to maintain fixation on this mark throughout the functional scanning. The fixation target was a small arrowhead $(0.5 \mathrm{deg})$ pointing in one of four directions (i.e., up, down, left, or right) which randomly changed direction every $4 \mathrm{~s}$. In order to aid fixation stability and maintain attention to the stimulus, subjects were given a task of monitoring and reporting the appearance of the fixation point. Subjects used a fiber-optic button pad with four buttons configured in a cross shape to report arrow orientation.

\subsubsection{Experiment 1: Hemifield Mapping}

This experiment utilized a blocked design, showing $16 \mathrm{~s}$ periods of a high-contrast, radially moving wedge in the left or right visual hemifield, alternating with a fixation-only condition. The wedge spanned either 100 degrees (40 degrees from vertical meridian) or 160 degrees (10 degrees from vertical meridian), and spared the central 0.5 degrees of visual space, 
where the fixation target was presented (Fig. 4-1 A). These stimuli were designed to avoid the cortical representation of the vertical meridian (the region of visual cortex where the hemifields overlap), similar to those used in an earlier study of the ipsilateral visual field representation in subjects with normal vision (Tootell et al., 1998). In this study, we report data from the hemisphere contralateral to the stimulated field. Each scan stimulated only one eye, consisting of 8 alternating blocks of hemifield stimulus and fixation, for a total scan length of 256 seconds. Eight total runs were collected, two for each combination of wedge size and eye. The entire scanning experiment lasted approximately 2 hours.

\subsubsection{Experiment 2: Retinotopic Mapping}

The cortical representation of retinotopic visual space was determined using a phaseencoded design in which the cardinal axes of visual space (eccentricity and polar angle) were mapped separately (Engel et al., 1997). The stimuli consisted of two different high-contrast, multi-colored checkerboard patterns. The "rotating wedge" stimulus sweeps through the polar angle dimension like the hand of a clock, while the "expanding ring" stimulus maps eccentricity by starting from the center of the visual field and expanding outward (Fig. 4-1 B). Eccentricity stimuli traversed space with a logarithmically increasing rate, as has been used previously (Conner et al., 2004; Sereno et al., 1995). This allowed the eccentricity stimulus to spend a greater proportion of the stimulus cycle in the foveal portion of the visual field, approximately accounting for cortical magnification from central vision to the periphery. Both stimuli also approximately compensated for the increasing receptive field size by increasing in size as they approach the periphery.

These phase-encoded stimuli used a 64 s cycle, completing eight cycles per scan. As a result, each functional scan took $512 \mathrm{~s}$, collecting 128 time points per functional scan volume. Four scans of this type were administered for each eye in one session, two for eccentricity and two for polar angle. Paired clockwise/counterclockwise and expansion/contraction scans were used in order to cancel the effects of residual hemodynamic phase delays. The entire scanning protocol for the retinotopic mapping experiment typically lasted about 2 hours. 


\subsubsection{Statistical Analysis}

The functional analysis was completed using the FS-FAST software tools freely available

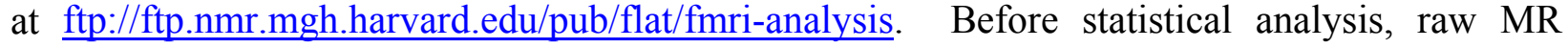
images were first motion-corrected to the $64^{\text {th }}$ volume (i.e., the midpoint of 128 volumes) of the first run using an iterated linearized weighted least squares method through the FS-FAST implementation of the AFNI 3dvolreg algorithm (Cox and Jesmanowicz, 1999). In addition to motion-correcting, this algorithm also provides a sum-of-squares estimate of average head motion throughout the fMRI scanning session, which was compared between subject groups for both experiments. The MR volumes were subsequently intensity normalized using the average in-brain voxel intensity. The resulting data sets were then used as inputs for the subsequent analysis, which differed for the two experiments.

\subsubsection{Experiment 1: Hemifield Mapping}

Briefly, the FS-FAST software was used to selectively average the blocks corresponding to stimulation of each of the left and right visual hemifields, separately for each eye. A voxelwise F-test computed the significance of the contrast between BOLD signal for each block and fixation baseline. The functional results were then resampled onto individual cortical surfaces, producing maps of statistical significance overlaid on inflated or flattened representations of individual brains. This allowed the pattern of significant $(p<0.05)$ fMRI results to be easily visualized for each subject.

\subsubsection{Experiment 2: Retinotopic Mapping}

Fast Fourier transform analysis was conducted on the time series of each voxel to statistically correlate retinotopic stimulus location with visual cortical anatomy. This analysis rejected low frequencies due to head motion or baseline drift and extracted functional signals in the form of magnitude and phase relative to the stimulus cycle frequency. Signal magnitude reflects retinotopic specificity, which can be low due to either lack of visually induced response or equivalent response to all retinotopic locations. The phase component of the signal codes specific retinotopic location, but is also dependent upon individual hemodynamic delay. To standardize the effect of heterogeneous hemodynamics across subjects, individual maps were first optimized with respect to phase for each subject's fellow (or dominant) eye, and the 
resulting phase offset was subsequently applied to the amblyopic eye maps. For eccentricity maps, this consisted of choosing a phase offset that optimally aligned the representation of central vision with the occipital pole, resulting in a normal map with a concentric red (foveal), blue (parafoveal), and green (peripheral) pattern. Thus, the fellow eye was established to serve as an internal control for each subject. The data were projected onto flattened representations of individual brains using a tricolor code of signal phase, i.e., visual field location. Finally, data from the eccentricity and polar angle scans were combined to create field sign maps for each eye, demonstrating visual area boundaries. The field sign maps' polarity is either similar or mirror symmetrical to the actual visual field geometry, and is defined as the sign of the cross-product between receptive field eccentricity and polar angle gradients (Sereno et al., 1994; Sereno et al., 1995).

In order to directly compare eccentricity stimulus magnitude patterns between eyes in individual subjects, we further plotted the significance of the F-statistic for each eye onto flattened occipital patches. These significance maps most accurately reflected the consistency of cortical activation in eccentricity scans, independent of phase. We used the Talairach Daemon (http://ric.uthscsa.edu/projects/talairachdaemon.html) to describe the location of any cortical regions with greater activity for amblyopic than for fellow eyes (Lancaster et al., 2000; Talairach and Tournoux, 1988).

\subsubsection{Region-of-Interest Analysis}

Field sign maps generated from each subject's fellow/dominant eye (Exp. 2) were used to define retinotopically specific regions-of-interest (ROIs) (Sereno et al., 1995; Engel et al., 1997). These objectively determined borders were available for V1 and V2 for all subjects, but further visual areas were not definable for most amblyopic subjects. In addition, a "foveal" ROI (FOV) was defined as the region of occipital pole activated by the fellow or dominant eye in the central 2.5 degrees of visual angle. ROIs corresponding to extrafoveal V1 and V2 (V1 $1_{\mathrm{EF}}$ and V2 $2_{\mathrm{EF}}$ ) were defined as the remainder of these areas (i.e., 2.5 to 15 degrees eccentricity) after subtraction of FOV.

For Exp. 1, these ROIs were used to extract BOLD signal magnitudes from the hemispheres contralateral to the stimulated hemifield for each experimental condition. These 
data were separately extracted for each ROI, wedge size, and eye. Mean signal magnitudes were then compared across diagnoses using a multifactorial, random-effects ANOVA. Initially, five factors were included in this analysis: subject group, ROI, wedge size, stimulated hemiretina (nasal vs. temporal), and eye (amblyopic vs. fellow eye or non-dominant vs. dominant eye). There were no significant effects of wedge size or hemiretina, so these factors were subsequently collapsed across conditions. Unpaired two-tailed $t$-tests were then used to directly compare amblyopic with control and fellow eyes for each subject group, within each ROI. For Exp. 2, the Fourier magnitude and phase of each vertex was extracted for each eye and ROI for the eccentricity stimulus. The average Fourier magnitudes were compared across diagnoses using a multifactorial, random-effects ANOVA with three factors: subject group, ROI, and eye.

Next, the Fourier phase values were analyzed for differences across groups. Phase values range from -180 to +180 , with negative values corresponding to foveal and positive values corresponding to peripheral field stimulation. In order to allow for noise in the phase estimation, correct for the phase discontinuity at the end of the eccentricity cycle (i.e., periphery wraps around to fovea), and avoid systematic bias in the mean phase estimation, the continuous circular phase scale was linearized and recentered to form a bracket around the predicted phase values for each ROI (Supplemental Fig. 4-S2 A). For the foveal ROI, the predicted phase of all vertices is 180 to -60 (the first third of the stimulus cycle), so phase values in the ROI corresponding to the last third of the stimulus cycle $(+60$ to +180$)$ were assumed to represent offset noise in the phase estimation and shifted by -360 , to -300 to -180 . This results in an effective range of -300 to +60 for the foveal ROI. Similarly, for $\mathrm{V} 1_{\mathrm{EF}}$ and $\mathrm{V} 2_{\mathrm{EF}}$, the predicted phase of all vertices is -60 to +180 (the final two-thirds of the stimulus cycle), so phase values corresponding to the first sixth of the stimulus cycle $(-180$ to -120$)$ were shifted by +360 , to +180 to +240 , resulting in an effective range of -120 to +240 (Supplemental Fig. 4-S1 B). This recentering procedure allowed the use of linear descriptive and comparative statistics for comparing phases across subjects. Mean phase was calculated for each ROI and eye in each subject. Interocular phase differences were then calculated (FE - AE or DE - NDE). Using this method, negative phase differences indicate a relative shift of amblyopic eye vertices toward later phase values (i.e., driven by more peripheral visual field locations) and vice versa. These average phase differences were compared across diagnoses for each ROI using an unpaired $t$-test. 
Finally, we recognized that reduced fixation stability in amblyopic subjects could influence our results, leading to possible misinterpretations. In order to dissociate our results from these effects, we performed a correlation analysis for BOLD signal magnitude (Exp. 1) and Fourier magnitude and phase (Exp. 2) with fixation stability during the corresponding scans.

\subsubsection{Interocular ROI Indices}

For Exp. 1, the ROIs defined above were also used to quantify the difference in fMRI response between eyes at the individual subject level. Two separate indices were defined, using only the cortical vertices that were significantly activated $(p<0.05)$.

Ocular Dominance Index. The dominance index (DI) was calculated as the difference between the number of vertices activated by each eye, divided by the sum of the number of vertices activated by each eye, i.e., $\mathrm{DI}=(\mathrm{FE}-\mathrm{AE}) /(\mathrm{FE}+\mathrm{AE})$. Using this calculation, $\mathrm{a}$ positive DI indicates more vertices activated by the fellow eye, while a negative DI indicates more vertices activated by the amblyopic eye. The non-dominant eye (NDE) and the dominant eye (DE) were used in place of $\mathrm{AE}$ and $\mathrm{FE}$ for control subjects. The mean DI for each subject group was compared with the control group using unpaired $t$-tests.

Binocularity Index. A vertex was classified as binocular if monocular stimulation through both eyes resulted in significant MR signal. Therefore, it should be noted that this index is not a true measure of binocularity, but rather and estimate of the potential cortical extent of binocularity. The binocularity index (BI) was thus calculated as the intersection divided by the union of vertices activated by each monocular stimulation, i.e., $\mathrm{BI}=(\mathrm{OS} \cap \mathrm{OD}) /(\mathrm{OS} \mathrm{U}$ OD) (Lee et al., 2001). Unlike Lee et al. (2001), who calculated the binocularity index using voxels in a volumetric ROI, our index was calculated using only vertices intersecting the cortical surface. The mean BI for each diagnosis group was compared with the control group using unpaired $t$-tests. 


\subsection{Results}

\subsubsection{Psychophysics}

\subsubsection{Grating Acuity}

Consistent with Snellen acuity (Table 1), grating acuity measures for fellow eyes were always better than for amblyopic eyes. This trend even held true for the control subjects, where 5/6 eyes classified as dominant had better grating acuity. Across groups, amblyopic subjects tended to have decreased grating acuity in their impaired eyes compared with both controls and their fellow eye $(C T L-D E=0.78 \mathrm{~min}, \mathrm{CTL}-\mathrm{NDE}=0.84 \mathrm{~min}$; STRAB-FE $=0.94 \mathrm{~min}, \mathrm{STRAB}-$ $\mathrm{AE}=1.49 \mathrm{~min} ;$ ANISO-FE $=0.84 \mathrm{~min}, \mathrm{ANISO}-\mathrm{AE}=1.24 \mathrm{~min}$ ). Consistent with previous work, amblyopic grating acuity exceeded Snellen acuity (Mayer et al., 1984; Mayer and Fulton, 1985), which is known to have additional sensitivity to higher-order effects such as crowding (Stuart and Burian, 1962; Levi et al., 2002).

\subsubsection{Contrast Sensitivity}

Contrast sensitivity in the amblyopic eyes of both groups was depressed compared with control eyes at 2, 4, and $8 \mathrm{cpd}$, consistent with the commonly reported higher spatial frequency deficit in amblyopia (Bodis-Wollner, 1980; Hess and Howell, 1977; Bradley and Freeman, 1981; McKee et al., 2003). These differences reached significance for anisometropic eyes at $4 \mathrm{cpd}$ $(\mathrm{CTL}-\mathrm{NDE}=39.8 \mathrm{~dB}$, ANISO-AE $=32.5 \mathrm{~dB}, t=4.96, p<0.05)$, but not at other spatial frequencies (Supplemental Fig. 4-S2 A). No significant differences were seen between contrast sensitivity functions for amblyopic vs. fellow eyes or for amblyopic fellow vs. control nondominant eyes for either patient group.

\subsubsection{Contour Integration}

Contour integration was significantly impaired for strabismic eyes compared with control eyes at the first $(\mathrm{CTL}-\mathrm{NDE}=96 \%$, STRAB-AE $=80 \%, t=3.67, p<0.05)$, second $(\mathrm{CTL}-\mathrm{NDE}=$ $92 \%$, STRAB-AE $=79 \%, t=2.93, p<0.05)$, third $(\mathrm{CTL}-\mathrm{NDE}=85 \%, \mathrm{STRAB}-\mathrm{AE}=67 \%, t=$ $4.81, p<0.01)$, and fifth (CTL-NDE $=61 \%$, STRAB-AE $=44 \%, t=3.85, p<0.05)$ difficulty levels. Furthermore, strabismic fellow eyes were also significantly impaired compared with control dominant eyes at the third (CTL-DE $=85 \%$, STRAB-FE $=71 \%, t=6.24, p<0.01$ ) and 
fifth $(\mathrm{CTL}-\mathrm{DE}=64 \%$, STRAB-FE $=48 \%, t=3.34, p<0.05)$ difficulty levels (Supplemental Fig. 4-S2 B). The strabismic and fellow eyes did not significantly differ from each other at any difficulty level. These results are consistent with reports of impairments at this task in both strabismic and fellow eyes, independent of acuity (Kovacs et al., 2000).

In contrast, no deficit was found for anisometropic eyes compared with control eyes, although an interocular comparison was significant at the third difficulty level (ANISO-FE = $88 \%$, ANISO-AE $=78 \%, t=4.89, p<0.01$ ), as has been shown elsewhere (Chandna et al., 2001). Previous work has suggested that strabismic and anisometropic amblyopia can be distinguished based on differences in higher-order visual functions such as vernier acuity and contour integration (Levi and Klein, 1982; Levi and Klein, 1985), so a greater impairment in strabismics was expected. Overall, the psychophysical results suggest that our strabismic and anisometropic subject groups are comparable to previous studies.

\subsubsection{Experiment 1: Hemifield Mapping}

\subsubsection{Fixation Stability and Head Motion}

In order to provide a single index of fixation during each scan, the standard deviation of horizontal gaze position was calculated for each subject. Vertical gaze position was also measured, but was less variable between subjects, consistent with the known pattern of fixational eye movements in amblyopes (Westall and Aslin, 1984; Ciuffreda et al., 1980). Mean gaze position variance was calculated from the raw eye position data after filtering for blinks. The means did not significantly differ between amblyopic and control eyes (CTL-NDE $=1.3 \mathrm{deg}$, $\mathrm{STRAB}-\mathrm{AE}=2.2 \mathrm{deg}$, ANISO-AE $=2.0 \mathrm{deg}$ ), although more variability existed between subjects in the amblyopic groups than in the control group (Fig. 4-2). Also, both amblyopic groups displayed a trend for less fixation stability than controls. Interestingly, two of the strabismic subjects actually had better fixation stability when viewing with their amblyopic eye (S2 and S4), but all anisometropic subjects had better stability when viewing with their fellow eyes. Control subjects had similar fixation stability in both dominant and non-dominant eyes. Review of fixation task response data confirmed that all subjects provided feedback for $>75 \%$ of the trials, with no significant differences in accuracy between the groups. Finally, head motion, 
estimated as the mean vector magnitude of translational motion within each scan, did not differ between eyes or groups.

\subsubsection{2 fMRI Activation Maps}

For both wedge sizes and both eyes, stimulation in one hemifield of visual space resulted in an increase in fMRI signal in the contralateral visual cortex in every subject, consistent with the normal retinogeniculocortical projection. In 4/6 strabismics and 5/6 anisometropes, a clear preference for the fellow compared with amblyopic eye was observable in both hemispheres in both the extent and the magnitude of the activation pattern. However, strabismics S2 and S5 did not show this pattern, instead exhibiting stronger activation from the nasal retina than the temporal retina of each eye. Additionally, the mild anisometrope A4 had stronger activation in both hemispheres in response to stimulation of the amblyopic than the fellow eye. In comparison, only $3 / 6$ control subjects displayed greater activation for the dominant vs. nondominant eye.

\subsubsection{ROI Analysis of Signal Magnitude}

The multifactorial ANOVA across groups revealed a main effect of eye, with greater mean signal elicited from the fellow/dominant eye $(\mathrm{FE} / \mathrm{DE}=0.57 \%, \mathrm{AE} / \mathrm{NDE}=0.44 \%, F=$ 23.97, $p<0.0001)$. Consistently greater signal was seen for $\mathrm{FE}$ or $\mathrm{DE}$ in most individual subjects as well (Fig. 4-3). The three exceptions (S2, S5, and A4) were noted above. At a group level, a trend for diminished signal magnitude for the weaker eye (AE/NDE) compared with the stronger eye (FE/DE) was present in every case, although none of the individual group level $t$ tests of amblyopic vs. fellow eye stimulation were significant (Table 2). Furthermore, there was a trend in every comparison for reduced mean signal magnitude in AE vs. CTL-NDE. This trend was significant for strabismic eyes compared with non-dominant control eyes in FOV (Table 2).

The comparison of nasal vs. temporal hemiretina did not show any significant differences, regardless of ROI, eye, or subject group. There was not even a consistent trend for greater signal magnitude from the nasal or temporal hemiretina across ROIs for any group. With the exception of the two strabismic subjects described above, there does seem to be any support for a hemiretinal bias in this group of subjects under the current viewing conditions. 
Finally, it was important to determine if fixation stability contributed to the signal magnitude differences, even though it did not significantly differ between groups. Thus, we tested for linear correlations between fixation stability and BOLD signal. We did find one significant correlation between signal magnitude in FOV and fixation variance for strabismic eyes $\left(\mathrm{R}^{2}=0.92, p<0.05\right)$, so in this case the reduced magnitude for strabismic eyes should be viewed with caution. However, none of the other ROIs $\left(\mathrm{V} 1_{\mathrm{EF}}\right.$ and $\left.\mathrm{V} 2_{\mathrm{EF}}\right)$ correlated with fixation for strabismics, nor did any of the ROIs for anisometropic subjects.

\subsubsection{ROI Interocular Indices}

Ocular Dominance Index. In general, individual strabismic and anisometropic subjects showed greater DI relative to control subjects for all ROIs (Fig. 4-4). The DIs for the control subjects were distributed tightly around zero, indicating no consistent ocular dominance, at least in number of activated vertices. In contrast, all but three of the amblyopic subjects showed fellow eye dominance, with positive DIs in all ROIs. Consistent with the ROI magnitude results, the exceptions were S2, S5, and A4, each with a DI favoring their amblyopic eye.

Despite the variability of individual subjects, all three ROIs for both amblyopic groups had mean DIs which were significantly more positive than controls (Table 3). Although DI was calculated from signal extent (number of vertices), it also strongly correlated across all subjects with an analogously calculated signal magnitude measure in FOV $\left(\mathrm{R}^{2}=0.74, p<0.00001\right), \mathrm{V} 1_{\mathrm{EF}}$ $\left(\mathrm{R}^{2}=0.73, p<0.00001\right)$, and $\mathrm{V} 2_{\mathrm{EF}}\left(\mathrm{R}^{2}=0.68, p<0.0001\right)$, thus supporting the known relationship between extent of activation and signal magnitude.

Binocularity Index. A wide range of individual BI values were seen for strabismic and anisometropic subjects, overlapping somewhat with the BI distribution for control subjects (Fig. 4-5). However, mean BIs for all ROIs of both amblyopic groups trended toward smaller values than controls, reaching significance for $\mathrm{V} 1_{\mathrm{EF}}$ and $\mathrm{V} 2_{\mathrm{EF}}$ of the anisometropic group (Table 3).

\subsubsection{Experiment 2: Eccentricity Mapping}

4.4.3.1 Fixation Stability and Head Motion 
As in Exp. 1, mean horizontal fixation stability did not significantly differ between amblyopic and control eyes $(\mathrm{CTL}-\mathrm{NDE}=3.1 \mathrm{deg}, \mathrm{STRAB}-\mathrm{AE}=3.7 \mathrm{deg}$, ANISO-AE $=2.2$ deg). In fact, one strabismic subject (S5) and three anisometropic subjects (A1, A3, and A4) actually had better fixation stability when viewing with their amblyopic than their fellow eye (Fig. 4-6). Review of fixation task response data for this experiment confirmed that all subjects provided feedback for $>95 \%$ of the trials, with no significant differences in accuracy between groups or eyes. Head motion did not differ between eyes or groups.

\subsubsection{2 fMRI Activation Maps}

Phase-encoded maps of eccentricity are shown for strabismic and anisometropic subjects (Fig. 4-7, control subjects are shown in Supplemental Fig. 4-S3). These individual maps of eccentricity are vertically arranged according to a qualitative assessment of departure from the normal pattern, with the more abnormal activation patterns placed toward the bottom. Normal eccentricity maps are comprised of a concentrically-organized pattern of red for central vision, blue for parafovea/mid-periphery, and green for periphery. S1 and A1, for example, possess eccentricity maps resembling the normal pattern for their fellow eyes. In general, the amblyopic eyes' maps were always less normal in appearance. Moreover, subjects near the bottom produced grossly abnormal maps, including a lack of concentric organization (S4, S5, S6, and A6) and a clearly diminished representation of foveal signals (fewer vertices labeled red for nearly all amblyopic eyes to some degree, but strikingly so for strabismics S4, S5, and S6, and anisometropes A5 and A6). These abnormalities usually occurred in concert with fellow eye maps which demonstrated a normal pattern of organization, but moderately diminished strength of signal.

\subsubsection{ROI Analysis of Fourier Magnitude}

Similar to Exp. 1, amblyopic eyes also produced lower Fourier magnitudes than either fellow or control eyes in most subjects and ROIs (Fig. 4-8). The exceptions were S6 (FOV), A3 $\left(\mathrm{V} 2_{\mathrm{EF}}\right), \mathbf{A} 4(\mathrm{FOV})$, and $\mathbf{A 6}\left(\mathrm{FOV}, \mathrm{V} 1_{\mathrm{EF}}\right.$, and $\left.\mathrm{V} 2_{\mathrm{EF}}\right)$. Despite the few exceptions, the multifactorial ANOVA for Exp. 2 resulted in a main effect of eye $(\mathrm{FE} / \mathrm{DE}=2.0, \mathrm{AE} / \mathrm{NDE}=$ $1.3, F=35.19, p<0.0001)$, as well as an interaction of diagnosis and eye $(F=4.74, p<0.01)$. However, upon separating the diagnoses, this effect remained significant only in $\mathrm{V} 1_{\mathrm{EF}}$ and $\mathrm{V} 2_{\mathrm{EF}}$ 
of strabismic subjects (Table 2). Finally, the ANOVA found an additional main effect of ROI $\left(\mathrm{FOV}=1.4, \mathrm{~V} 1_{\mathrm{EF}}=1.5, \mathrm{~V} 2_{\mathrm{EF}}=2.0, F=9.25, p<0.001\right)$, reflective of a trend for greatest Fourier magnitude in $\mathrm{V} 2_{\mathrm{EF}}$ in all groups.

When we compared the amblyopic eyes to control eyes, we found a trend for less Fourier magnitude in amblyopic eyes. Between-group t-tests revealed a significant deficit in $\mathrm{V} 1_{\mathrm{EF}}$ of anisometropic eyes (Table 2), but the clear trends suggest that a larger number of subjects would result in significant $t$-test comparisons in more of the ROIs of both amblyopic groups. Finally, Fourier magnitude did not correlate with fixational variance in any group or ROI.

\subsubsection{ROI Analysis of Eccentricity Phase}

The results of this quantitative analysis of Fourier signal phase were consistent with the qualitative observation that both amblyopic groups demonstrated reduced cortical representations of foveal stimuli from their amblyopic compared with their fellow eyes (Fig. 4-9). Mean phase differences between the fellow and amblyopic eyes (FE - AE) in FOV were significantly shifted toward more negative values (i.e., greater eccentricities) for both strabismic and anisometropic subjects (Table 3). Notably, neither group differed significantly from controls in $\mathrm{V} 1_{\mathrm{EF}}$ or $\mathrm{V} 2_{\mathrm{EF}}$. Importantly, eccentricity phase did not correlate with fixation stability for either anisometropic or strabismic groups, suggesting that this result is relatively insensitive to small eye movements. For example, the fellow eye of S5 had the worst fixation stability in the study, yet produced a normal eccentricity map. Finally, there were no significant differences across groups in the proportion of recentered vertices, arguing against the potential explanation that the mean phase shift is noise-related. Instead, the preferred eccentricity shift shown here suggests cortical remapping, i.e., interocular differences in the strength of representation of visual field locations.

\subsubsection{5 fMRI Patterns in Higher-Order Cortex}

Although the previous ROI analyses allowed quantitative assessment of interocular differences in fMRI signal in early retinotopic areas, they do not address the possibility of differences in higher-level cortical regions. Qualitatively, a few subjects' eccentricity maps appeared to have greater signal in parietal and temporal cortical regions for amblyopic than for fellow eye stimulation. We plotted the significance of the F-statistic, and unexpectedly found three amblyopic subjects with increased fMRI activation in regions of parietal and temporal 
cortex for amblyopic compared with fellow eye viewing (Fig. 4-10, summarized with Talairach coordinates in Table 4). This pattern was not seen in any control subjects. Specifically, the right hemisphere of strabismic S4 showed greater activation in the middle segment of the intraparietal sulcus (IPS), while S5 demonstrated bilaterally increased activation in the posterior segment of the IPS. Anisometrope A2 also showed more activation in the posterior segment of the left IPS and middle segment of the right IPS, combined with increased signal in the fusiform gyrus of the right hemisphere. It should be noted that all three of these subjects had greater activation in all retinotopic ROIs when stimulated through the fellow eye (Fig. 4-8), dissociating these high-level findings from patterns in lower retinotopic ROIs. We consider possible explanations for these results in the discussion. 


\subsection{Discussion}

We have demonstrated that amblyopia and the associated interruption of early visual experience leads to regionally specific abnormalities in neural processing of visual stimuli in human subjects. This is the first study to directly measure retinotopic organization driven by amblyopic eyes relative to both fellow and control eyes. Our study improved upon previous work by using a relatively large number of subjects, monitoring fixation, and individually defining retinotopic ROIs on the flattened cortical surface.

\subsubsection{Fixation Stability}

Given evidence that fixation stability can be impaired in amblyopia (Westall and Aslin, 1984), it is an important potential confound. However, we did not find significantly impaired fixation stability in our amblyopes. Furthermore, fixation task performance was equivalent across groups, further supporting our claim that fixation and attention were maintained throughout the experiment. These measurements suggest that impaired fixation does not necessarily preclude meaningful fMRI experiments in mild-to-moderate amblyopic subjects. Finally, the lack of correlations between fixation stability and fMRI measures in all but one case (Exp. 1, STRAB, FOV) provides further argument against eye movements as a confounding factor.

\subsubsection{Signal Magnitude Deficits}

We report here a main effect of eye for both experiments, despite our stringent inclusion of eye dominance in control subjects. It is notable that acuity-based eye dominance in controls was an effective predictor of interocular signal magnitude (Fig. 4-3), a novel result. We do not claim that our method of assigning eye dominance extends to all visual functions, but this implementation provided a conservative control for amblyopia. The main effects of eye were apparent as group-level trends for all ROIs of both amblyopic subtypes, consistent with previous PET (Demer et al., 1988; Demer et al., 1997; Imamura et al., 1997) and fMRI (Barnes et al., 2001; Choi et al., 2001; Algaze et al., 2002). Furthermore, amblyopic eyes showed group-level trends for less signal than control eyes. We found significantly reduced signal magnitude in 
FOV of strabismic eyes (Fig. 4-3), which appeared to support the hypothesis of impaired central vision, but fixation stability predicted this fMRI signal. However, signal magnitudes in the strabismics' other ROIs did not correlate with eye movements. One explanation for this dissociation is that FOV was more susceptible to small eye movements than $\mathrm{V} 1_{\mathrm{EF}}$ or $\mathrm{V} 2_{\mathrm{EF}}$, presumably due to larger magnification factor in foveal neurons. Regardless, it is clear that future PET or fMRI amblyopia studies should monitor eye movements and consider subject training to stabilize fixation.

\subsubsection{Shifted Ocular Dominance}

Although human post-mortem cases have not found abnormal ocular dominance column (ODC) periodicity (Horton and Stryker, 1993; Horton and Hocking, 1996), two recent neuroimaging studies demonstrated ocular dominance shifts in V1 of human amblyopes. Goodyear et al. (2002) used high resolution $(0.5 \times 0.5 \times 3 \mathrm{~mm})$ fMRI to image ODCs in six strabismic adults (Goodyear et al., 2002), finding a 60/40 dominance ratio in favor of the fellow eye. Alternatively, Liu et al. (2004) demonstrated dominance for the fellow eye in two anisometropic amblyopes, but instead constructed ocular dominance histograms (Hubel and Wiesel, 1962) using the voxel-wise Student $t$-statistic for the OS vs. OD contrast (Liu et al., 2004). We used a vertex-counting technique similar to Goodyear et al. (2002), but did not attempt to resolve individual ODC boundaries. Like Liu et al. (2004), our method pooled signals across multiple ODCs to compute mean ocular dominance in larger cortical regions. Our results agree well with these previous reports.

\subsubsection{Impaired Cortical Binocularity}

Cortical binocularity has been extensively studied in humans with amblyopia using psychophysics. These studies have demonstrated impaired binocular summation, but intact inhibition (i.e., suppression or dichoptic masking) in amblyopes (Levi et al., 1980; Harrad and Hess, 1992). Visual evoked potentials (VEPs) have confirmed this dissociation in both strabismic and anisometropic amblyopes (Tsutsui and Fukai, 1980; Baitch and Levi, 1988).

A recent pair of fMRI papers has added to these physiological results. Using a binocularity index similar to ours, Lee et al. (2001) reported impaired binocularity for strabismic 
$(\mathrm{N}=6)$ and anisometropic $(\mathrm{N}=5)$ amblyopes in the calcarine region, while Algaze et al. (2002) found reduced calcarine binocularity for 5 amblyopic subjects of mixed diagnoses. We improved upon these techniques by using vertices restricted to the cortical surface, in multiple visual areas. We found degraded binocularity in our amblyopes, although not as severe as previously reported. Since we did not use a binocular viewing condition, we instead divided the intersection by the union of monocularly driven vertices. Our index does not account for the possibility of interocular suppression under natural viewing conditions, so we potentially overestimated binocularity. Furthermore, we cannot completely rule out interocular suppression with our viewing conditions, but our fixation task and stability data suggest that our amblyopic subjects were not suppressing the central fixation target, where suppression of the amblyopic eye tends to be most severe (Sireteanu, 1982). Future studies employing other viewing conditions (e.g., fellow eye open or closed) could be informative.

\subsubsection{Nasotemporal Asymmetry}

Amblyopia psychophysics have shown consistently greater impairments for the nasal retina, e.g., visual acuity and luminance detection (Sireteanu and Fronius, 1981), reaction time for suprathreshold light detection (Chelazzi et al., 1988), and pursuit eye movements and monocular optokinetic nystagmus (OKN) (Tychsen and Lisberger, 1986; Schor and Levi, 1980). These findings have been postulated to result from constant, active suppression of input from the amblyopic eye's nasal retina by the foveal region of the fellow eye's temporal retina under binocular viewing conditions (Sireteanu and Fronius, 1990). We thus hypothesized that nasal retina stimulation might result in less fMRI activation. However, we found no evidence for this effect with our current methods. Future studies of this issue could adopt viewing conditions that encourage greater interocular suppression or focus more specifically on the oculomotor system.

\subsubsection{Visual Field Remapping}

Rapid reorganization of visual cortex has been demonstrated after focal retinal (Chino et al., 1992; Chino et al., 1995) and cortical lesions (Eysel and Schweigart, 1999; Zepeda et al., 2003), and in one study foveal lesions remapped more quickly and effectively than lesions in

peripheral retina (Rosa et al., 1995). Furthermore, the literature is rich with examples of 
learning-related psychophysical improvements in amblyopia (Levi and Polat, 1996; Polat et al., 2004) and reorganization in patients with congenital photoreceptor abnormalities and brain lesions (Baseler et al., 1999; Baseler et al., 2002). Therefore, we hypothesized that active cortical remapping could occur in the central field representation of early visual areas in amblyopia.

We indeed found significant shifts toward greater preferred eccentricity in FOV of amblyopic eyes, indicating that foveal eccentricities in the fellow eye and parafoveal eccentricities in the amblyopic eye shared cortical area, reminiscent of the effects of a central retinal lesion. Although amblyopia cannot be equated with retinal denervation, cortical reorganization is plausible following years of chronic suppression of foveal input from the amblyopic eye under normal viewing conditions. Moreover, we found no relationship between fixation stability and eccentricity remapping, making such an explanation unlikely.

\subsubsection{Findings in Temporal and Parietal Cortex}

We observed greater activity for amblyopic than fellow eye stimulation in regions of parietal and ventral temporal cortex in three amblyopic subjects (S4, S5, and A2), a pattern not seen in any control subjects. It is notable that this activity was dissociable from effects in retinotopic cortex, since all three subjects showed the expected amblyopic eye impairment in V1 and V2.

All three subjects had abnormal activity with amblyopic eye stimulation in mid-posterior IPS, homologous to monkey lateral intraparietal area (LIP). This was seen in the right hemisphere of S4 and bilaterally in S5 and A2. We propose two possible explanations. First, this result may reflect an input "bottleneck" to visual cortex. We have already shown reduced $\mathrm{V} 1_{\mathrm{EF}}$ and $\mathrm{V} 2_{\mathrm{EF}}$ activity, but LIP receives diffuse projections from both retinotopic cortex and subcortical structures such as the superior colliculus and pulvinar (Lewis and Van Essen, 2000; Blatt et al., 1990). With the depression of input from V1 and V2, disinhibition of subcortical pathways could lead to increased LIP activity. A second possibility is that this activity might result from increased attentional effort when viewing with the amblyopic eye (Corbetta and Shulman, 2002). LIP has been termed the "parietal eye field" because of its role in fixation and

eye movement planning (Cavada, 2001). Impaired control of ocular motility might require 
increased vigilence, leading to greater activation in both the LIP and the frontal eye fields (FEF). A future study of frontal areas in amblyopia might help validate this hypothesis.

We also found increased activation in $\mathbf{A} 2$ of the right posterior fusiform gyrus of the ventral temporal lobe, a region previously implicated in object processing (Malach et al., 1995; Lerner et al., 2003) and orientation discrimination (Faillenot et al., 2001). This increased activation might also reflect attentional effort for amblyopic eye viewing and performance of the orientation discrimination task.

Finally, structural abnormalities in very similar regions have been recently described in amblyopia. Statistical analyses have found reduced gray matter volume in the IPS of strabismic adults (Chan et al., 2004) and in the IPS and fusiform gyrus of anisometropic and strabismic children (Mendola et al., 2005). The convergence of structural and fMRI results is noteworthy. It would be valuable to study these cortical areas in more detail with animal models of amblyopia. 


\subsection{Conclusions}

We conclude that functional abnormalities of visual cortical areas are a prevalent marker of human amblyopia. We used fixation monitoring and retinotopic ROIs, methods that should prove useful in further studies of amblyopia. We replicated the results of others with our BOLD signal magnitude measures, but show that these effects are of moderate size when strictly compared to normal controls. Unlike some previous reports, our results do not clearly discriminate the two amblyopic subtypes. Retinotopic mapping of the amblyopic eye showed a shift of parafoveal eccentricity representations toward the occipital pole. Finally, we also found novel activation patterns in parietal and temporal cortex in several amblyopic subjects, suggesting future avenues of amblyopia research. 


\subsection{Tables}

Table 1. Subject Characteristics

\begin{tabular}{|c|c|c|c|c|c|c|c|c|c|c|}
\hline $\begin{array}{l}\text { CTL } \\
(\mathrm{N}=6)\end{array}$ & $\begin{array}{c}\text { Age at } \\
\text { Diagnosis } \\
\text { (years) }\end{array}$ & $\begin{array}{c}\text { Patch } \\
\text { Duration } \\
\text { (months) }\end{array}$ & $\begin{array}{l}\text { Age at } \\
\text { Surgery } \\
\text { (years) }\end{array}$ & $\begin{array}{l}\text { Current } \\
\text { Deviation } \\
\text { (prism } \\
\text { diopters) }\end{array}$ & NDE & $\begin{array}{l}\text { NDE } \\
\text { VA } \\
\text { (MAR) }\end{array}$ & $\begin{array}{c}\text { Near } \\
\text { VA Diff } \\
\text { (MAR) }\end{array}$ & $\begin{array}{c}\text { Far } \\
\text { VA Diff } \\
(M A R)\end{array}$ & $\begin{array}{c}\text { IRD } \\
\text { (diopters) }\end{array}$ & $\begin{array}{c}\text { Stereo- } \\
\text { acuity } \\
\text { (arc-sec) }\end{array}$ \\
\hline C1 & - & - & - & - & OS & 1.25 & 0.00 & 0.00 & - & 40 \\
\hline$\bullet \mathrm{C} 2$ & - & - & - & - & OD & 1.00 & 0.20 & 0.00 & - & 40 \\
\hline$\Delta \mathrm{C} 3$ & - & - & - & - & os & 1.00 & 0.00 & 0.00 & - & 40 \\
\hline$\times \mathrm{C} 4$ & - & - & - & - & OD & 1.00 & 0.00 & 0.00 & - & 40 \\
\hline * C5 & - & - & - & - & OS & 1.00 & 0.20 & 0.20 & - & 80 \\
\hline - $\mathrm{C} 6$ & - & - & - & - & OD & 1.60 & 0.35 & 0.25 & - & 40 \\
\hline Mean & - & - & - & - & - & 1.14 & 0.13 & 0.08 & - & - \\
\hline $\begin{array}{c}\text { STRAB } \\
(\mathrm{N}=6)\end{array}$ & $\begin{array}{c}\text { Age at } \\
\text { Diagnosis } \\
\text { (years) }\end{array}$ & $\begin{array}{c}\text { Patch } \\
\text { Duration } \\
\text { (months) }\end{array}$ & $\begin{array}{l}\text { Age at } \\
\text { Surgery } \\
\text { (years) }\end{array}$ & $\begin{array}{l}\text { Current } \\
\text { Deviation } \\
\text { (prism } \\
\text { diopters) }\end{array}$ & $\mathrm{AE}$ & $\begin{array}{c}\text { AE } \\
\text { VA } \\
(M A R)\end{array}$ & $\begin{array}{l}\text { Near } \\
\text { VA Diff } \\
\text { (MAR) }\end{array}$ & $\begin{array}{l}\text { Far } \\
\text { VA Diff } \\
\text { (MAR) }\end{array}$ & $\begin{array}{c}\text { IRD } \\
\text { (diopters) }\end{array}$ & $\begin{array}{c}\text { Stereo- } \\
\text { acuity } \\
\text { (arc-sec) }\end{array}$ \\
\hline S1 & 3 & 24 & - & 20 & OD & 1.60 & 0.60 & 0.80 & 0.75 & $>3500$ \\
\hline s2 & 4 & 6 & 15 & 20 & os & 1.25 & 0.25 & 0.60 & 0 & $>3500$ \\
\hline$\Delta \mathrm{S} 3$ & 6 & 2 & - & 6 & OD & 2.00 & 1.00 & 1.00 & 0.75 & 400 \\
\hline$\times S 4$ & 2 & 36 & 2,13 & 12 & os & 2.50 & 1.50 & 1.50 & $2.75^{\star}$ & $>3500$ \\
\hline * S5 & 10.5 & 0 & - & 25 & OD & 2.50 & 1.25 & 1.25 & 0 & $>3500$ \\
\hline S6 & 4 & 24 & 6 & 8 & OS & 8.00 & 7.00 & 7.00 & 1.0 & 3500 \\
\hline Mean & 4.9 & 15.3 & - & 11.8 & - & 2.98 & 1.93 & 2.03 & 0.50 & - \\
\hline & & & & & & & & & & \\
\hline $\begin{array}{c}\text { ANISO } \\
(\mathrm{N}=6)\end{array}$ & $\begin{array}{c}\text { Age at } \\
\text { Diagnosis } \\
\text { (years) }\end{array}$ & $\begin{array}{c}\text { Patch } \\
\text { Duration } \\
\text { (months) }\end{array}$ & $\begin{array}{l}\text { Age at } \\
\text { Surgery } \\
\text { (years) }\end{array}$ & $\begin{array}{l}\text { Current } \\
\text { Deviation } \\
\text { (prism } \\
\text { diopters) }\end{array}$ & $\mathrm{AE}$ & $\begin{array}{c}\text { AE } \\
\text { VA } \\
\text { (MAR) }\end{array}$ & $\begin{array}{c}\text { Near } \\
\text { VA Diff } \\
\text { (MAR) }\end{array}$ & $\begin{array}{c}\text { Far } \\
\text { VA Diff } \\
\text { (MAR) }\end{array}$ & $\begin{array}{c}\text { IRD } \\
\text { (diopters) }\end{array}$ & $\begin{array}{c}\text { Stereo- } \\
\text { acuity } \\
\text { (arc-sec) }\end{array}$ \\
\hline$\square \mathrm{A} 1$ & 9 & 2 & - & - & OS & 4.00 & 2.75 & 2.15 & 3.5 & 800 \\
\hline$\rightarrow \mathrm{A} 2$ & 5 & 12 & - & - & OD & 2.00 & 0.75 & 1.25 & 1.75 & 400 \\
\hline$\Delta \mathrm{A} 3$ & 5.5 & 24 & - & - & OS & 2.00 & 1.00 & 0.60 & 2.0 & 800 \\
\hline$\times \mathrm{A4}$ & 10 & 2 & - & - & OD & 1.60 & 0.60 & 0.25 & 1.5 & 800 \\
\hline * A5 & 9 & 1 & - & - & OD & 1.25 & 0.25 & 0.80 & 2.75 & 100 \\
\hline A6 & 9 & 12 & - & - & OD & 10.00 & 8.00 & 6.00 & 0.5 & $>3500$ \\
\hline Mean & 7.9 & 8.8 & - & - & - & 3.48 & 2.23 & 1.84 & 2.00 & - \\
\hline
\end{tabular}

VA - visual acuity, reported as minimum angle of resolution (MAR) in arc-minutes.

IRD - interocular refractive difference, diopters of spherical equivalent.

NDE - non-dominant control eye, AE - amblyopic eye.

* IRD consistent with mixed strabismic/anisometropic diagnosis. 


\section{Table 2. Comparison of Signal Magnitude in Regions-of-Interest}

\begin{tabular}{|c|c|c|c|c|}
\hline & \multicolumn{2}{|c|}{ Exp. 1 - BOLD Signal } & \multicolumn{2}{c|}{ Exp. 2 - Fourier Signal } \\
\hline $\begin{array}{c}\text { STRAB } \\
\text { (N=6) }\end{array}$ & AE vs FE & AE vs CTL & AE vs FE & AE vs CTL \\
\hline FOV & $\checkmark$ & $0.05(2.28)$ & $\checkmark$ & $\checkmark$ \\
\hline V1 $1_{\mathrm{EF}}$ & $\checkmark$ & $\checkmark$ & $0.05(2.22)$ & $\checkmark$ \\
\hline V2 $2_{\mathrm{EF}}$ & $\checkmark$ & $\checkmark$ & $0.004(3.73)$ & $\checkmark$ \\
\hline & \multicolumn{5}{|c|}{} \\
\hline $\begin{array}{c}\text { ANISO } \\
\text { (N=6) }\end{array}$ & AE vs FE & AE vs CTL & AE vs FE & AE vs CTL \\
\hline FOV & $\checkmark$ & $\checkmark$ & $\checkmark$ & $\checkmark$ \\
\hline V1 $1_{\mathrm{EF}}$ & $\checkmark$ & $\checkmark$ & $\checkmark$ & $0.05(2.25)$ \\
\hline V2 2 EF & $\checkmark$ & $\checkmark$ & $\checkmark$ & $\checkmark$ \\
\hline
\end{tabular}

$\mathrm{AE}$ - amblyopic eye, FE - fellow eye.

$\mathrm{CTL}$ - non-dominant control eye.

FOV - foveal ROI, V1 $1_{E F}$ - extrafoveal V1, V2 ${ }_{E F}$ - extrafoveal V2.

$\checkmark$ indicates expected trend for reduced signal AE signal compared to FE or CTL. (Significant comparisons are given as $p(t)$ ) 
Table 3. Comparison of Magnitude Indices and Phase in Regions-of-Interest

\begin{tabular}{|c|c|c|c|}
\hline & \multicolumn{2}{|l|}{ Exp. 1} & Exp. 2 \\
\hline $\begin{array}{c}\text { STRAB } \\
(\mathrm{N}=6)\end{array}$ & $\begin{array}{c}\text { DI } \\
\text { vs } \mathrm{CTL}\end{array}$ & $\begin{array}{c}\mathrm{BI} \\
\text { vs } \mathrm{CTL}\end{array}$ & $\begin{array}{c}\text { Phase Diff } \\
\text { vs CTL }\end{array}$ \\
\hline FOV & $0.03(2.41)$ & $\checkmark$ & $0.03(2.74)$ \\
\hline $\mathrm{V} 1_{\mathrm{EF}}$ & $0.02(2.48)$ & $\checkmark$ & $x$ \\
\hline $\mathrm{V} 2_{\mathrm{EF}}$ & $0.03(2.32)$ & $\checkmark$ & $\checkmark$ \\
\hline $\begin{array}{c}\text { ANISO } \\
(\mathrm{N}=6)\end{array}$ & $\begin{array}{c}\text { DI } \\
\text { vs CTL }\end{array}$ & $\begin{array}{c}\mathrm{BI} \\
\text { vs } \mathrm{CTL}\end{array}$ & $\begin{array}{c}\text { Phase Diff } \\
\text { vs CTL }\end{array}$ \\
\hline FOV & $0.001(3.69)$ & $\checkmark$ & $0.03(2.55)$ \\
\hline $\mathrm{V} 1_{\mathrm{EF}}$ & $0.02(2.53)$ & $0.004(3.20)$ & $\checkmark$ \\
\hline $\mathrm{V} 2_{\mathrm{EF}}$ & $0.02(2.43)$ & $0.02(2.47)$ & $x$ \\
\hline
\end{tabular}

$\mathrm{DI}$ - dominance index, $\mathrm{BI}$ - binocularity index.

FOV - foveal ROI, V1 $1_{E F}$ - extrafoveal V1, V2 ${ }_{E F}$ - extrafoveal V2.

$\checkmark$ indicates expected trend for reduced BI (Exp. 1),

or greater interocular phase difference (Exp. 2) compared to CTL. (Significant comparisons are given as $p(t)$ )

$\mathbf{x}$ indicates trend for reduced interocular phase difference compared to CTL. 


\section{Table 4. Parietal and Temporal Activation in Amblyopic Subjects}

\begin{tabular}{|c|c|c|c|c|}
\hline $\begin{array}{c}\text { Subject } \\
\text { Identifier }\end{array}$ & Cortical Location & \multicolumn{3}{|c|}{$\begin{array}{ccc}\text { Talairach Coordinates } \\
\mathbf{X} & \mathbf{Y} & \mathbf{Z}\end{array}$} \\
\hline$\times \mathrm{S} 4$ & $\mathrm{RH}$ : middle IPS & +18 & -58 & +55 \\
\hline * S5 & $\begin{array}{l}\text { LH: posterior IPS } \\
\text { RH: posterior IPS }\end{array}$ & $\begin{array}{r}-25 \\
+25\end{array}$ & $\begin{array}{l}+61 \\
-66\end{array}$ & $\begin{array}{l}+35 \\
+31\end{array}$ \\
\hline A2 & $\begin{array}{l}\text { LH: posterior IPS } \\
\mathrm{RH}: \text { middle IPS } \\
\\
\text { posterior fusiform }\end{array}$ & $\begin{array}{r}-26 \\
+23 \\
+37\end{array}$ & $\begin{array}{l}-66 \\
-68 \\
-48\end{array}$ & $\begin{array}{r}+32 \\
+63 \\
-11\end{array}$ \\
\hline
\end{tabular}

IPS - intraparietal sulcus 


\subsection{Figures}
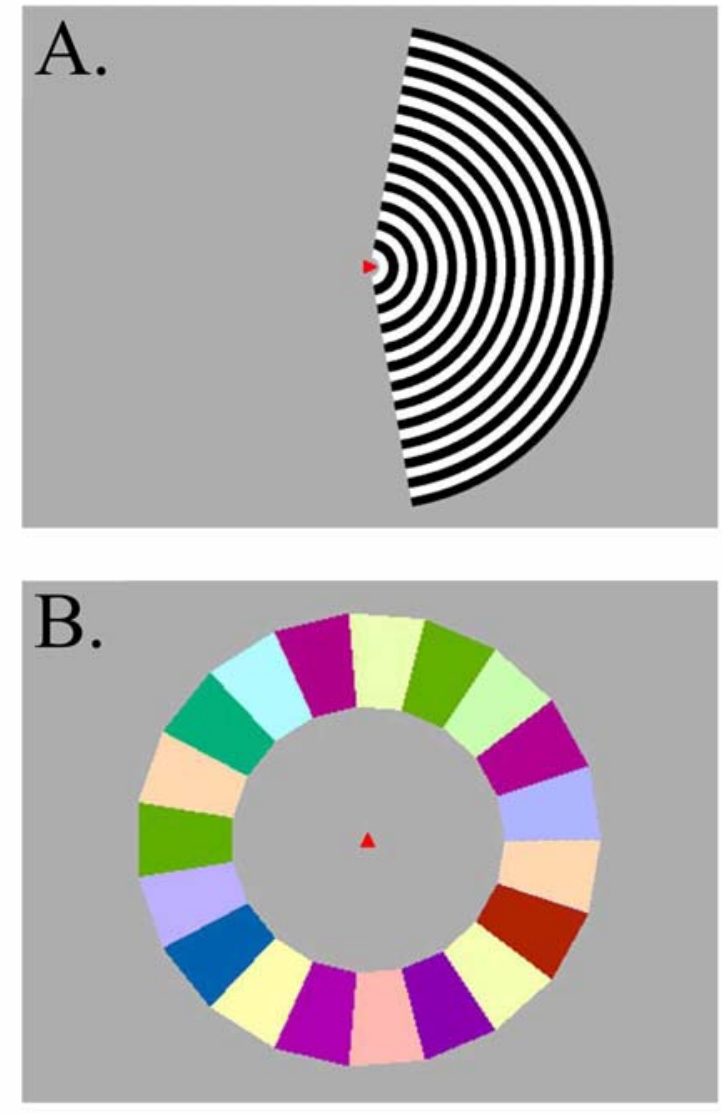

FIGURE 4-1. FMRI STIMULI. The subject maintained fixation on the central "arrowhead" target. Attention was monitored by recording a button-press each time the arrowhead changed orientation. A. The hemifield stimulus, measuring either 100 or 160 degrees, was presented in $16 \mathrm{~s}$ alternating blocks to the left and right hemifields. B. The eccentricity stimulus, which mapped retinotopic visual space, was presented in paired expansion and contraction scans. 


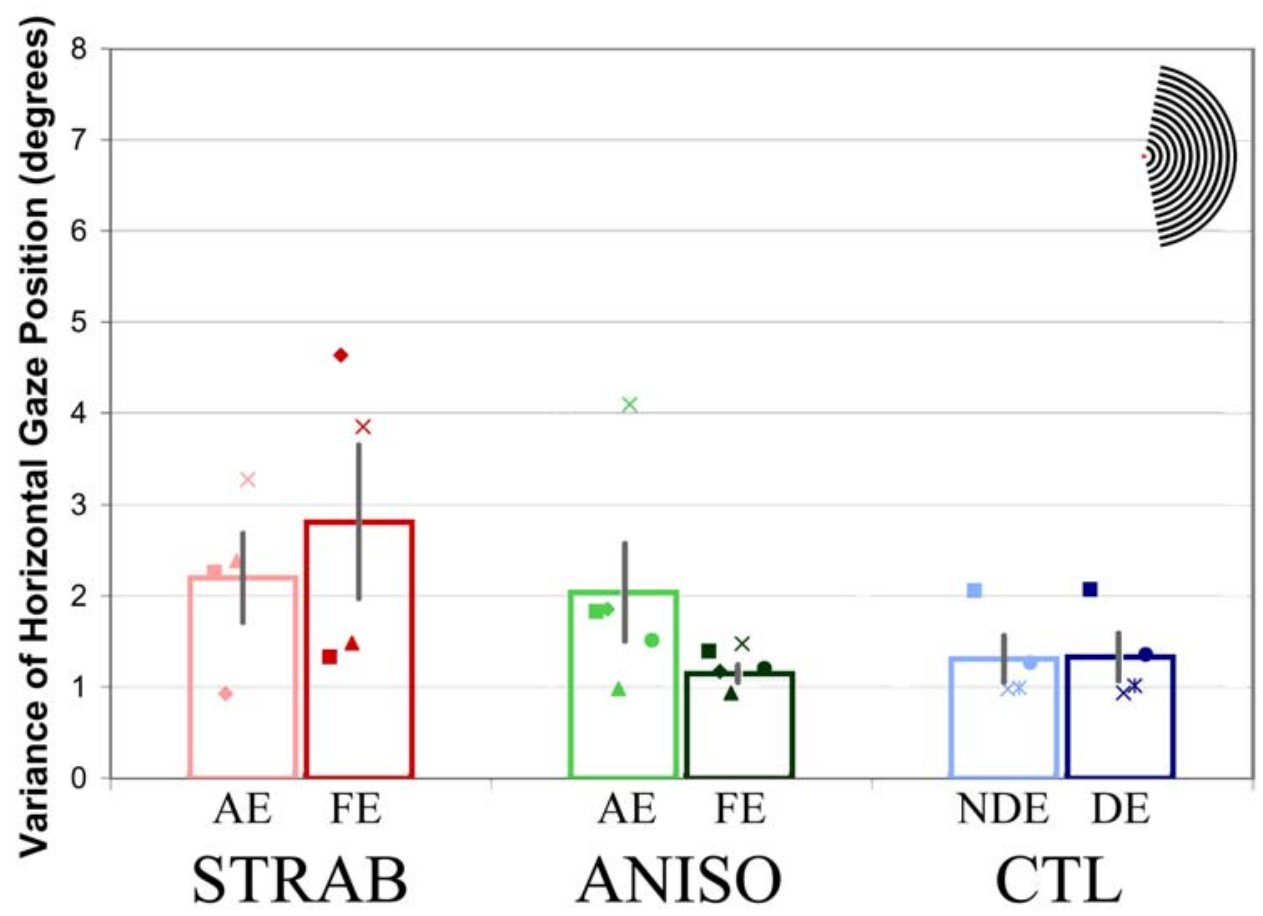

Figure 4-2. FiXAtion Stability FOR EXPERIMENT 1. No significant differences of fixation stability are seen for either intergroup or interocular comparisons. Group mean (open bars) and individual data are shown, with saturated colors corresponding to the dominant/fellow eye $(\mathrm{DE} / \mathrm{FE})$ and lighter shades to the non-dominant/amblyopic eye (NDE/AE). Calibration error resulted in lack of eye tracking data in two strabismics (S5, S6), one anisometrope (A5), and two controls $(\mathbf{C} 2, \mathbf{C} 3)$. Error bars show the standard error of the mean. 


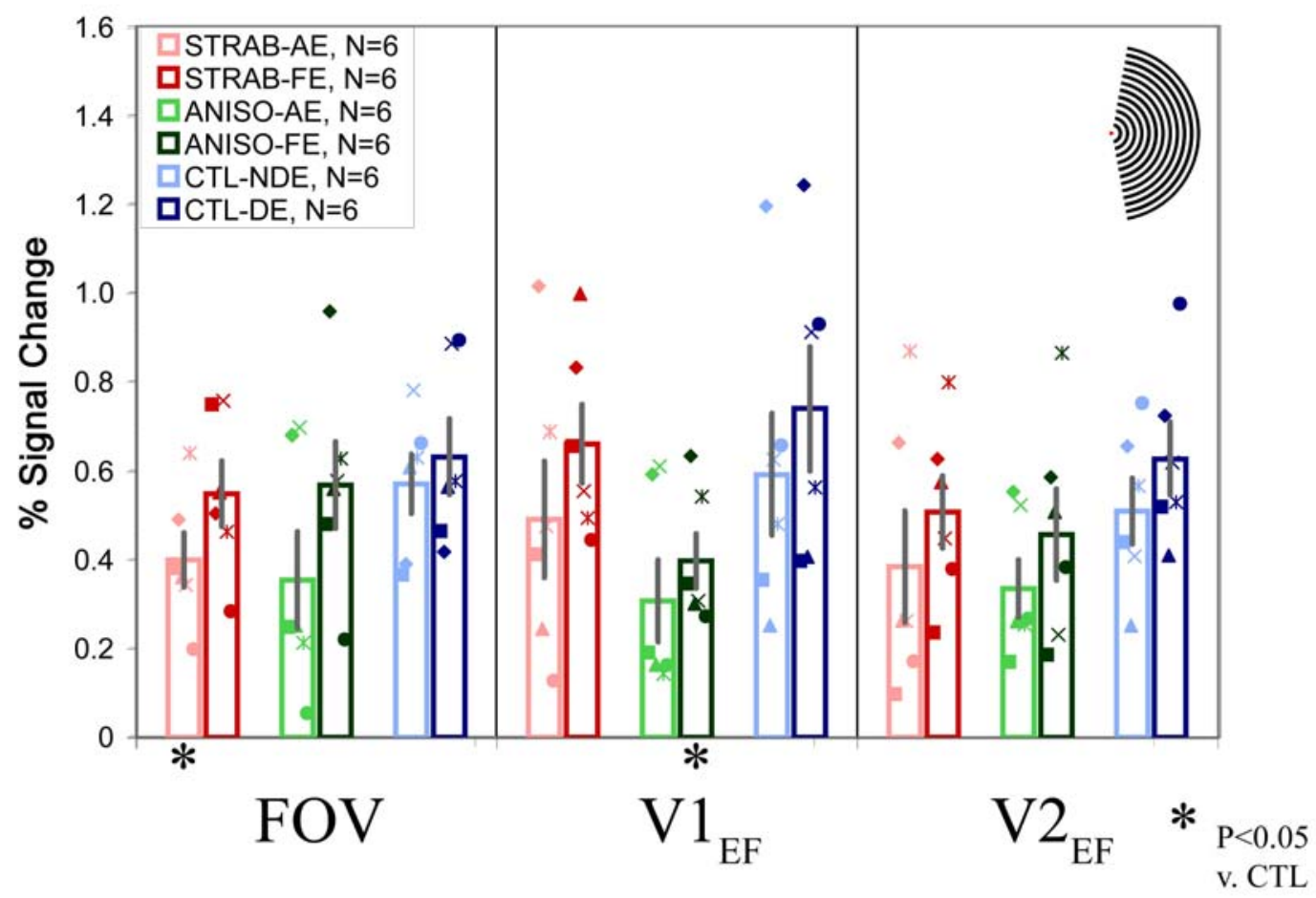

Figure 4-3. FMRI BOLD Signal MAgnitude FOR EXPERIMENT 1. Mean BOLD signal magnitude is depressed for every intergroup and interocular comparison, although a few amblyopic patients do not follow this trend (S2, S5, and A4, see text for details). There is also a consistent trend for less signal magnitude in NDE compared to DE for controls. Asterisks indicate significant intergroup differences. Other labeling conventions are the same as in Fig. 42. 


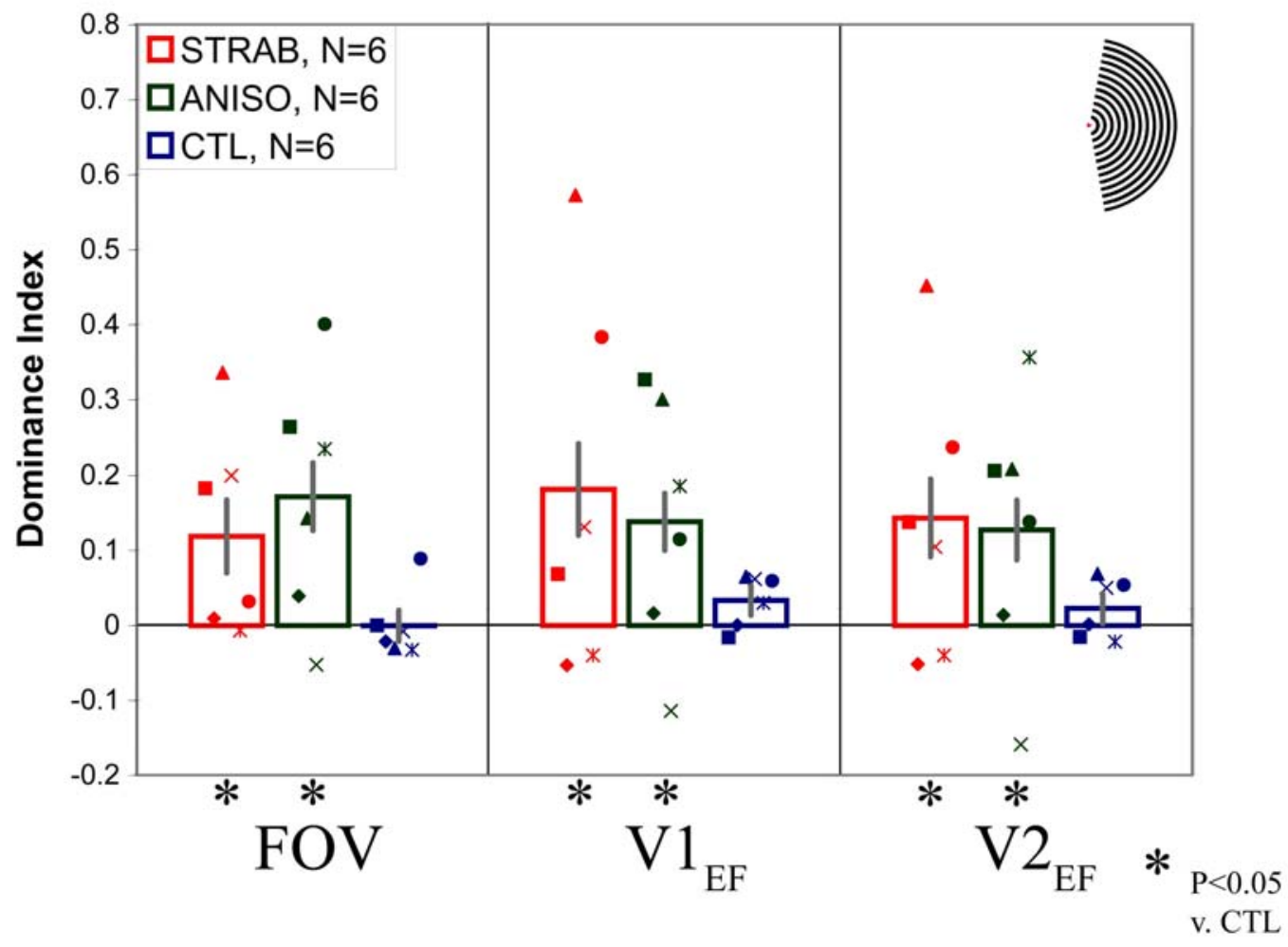

Figure 4-4. OCUlar Dominance INDEX By GROUP AND ROI. Mean DI is significantly shifted toward the fellow eye in all ROIs for strabismic and anisometropic amblyopes. Labeling conventions are the same as in Fig. 4-3. 


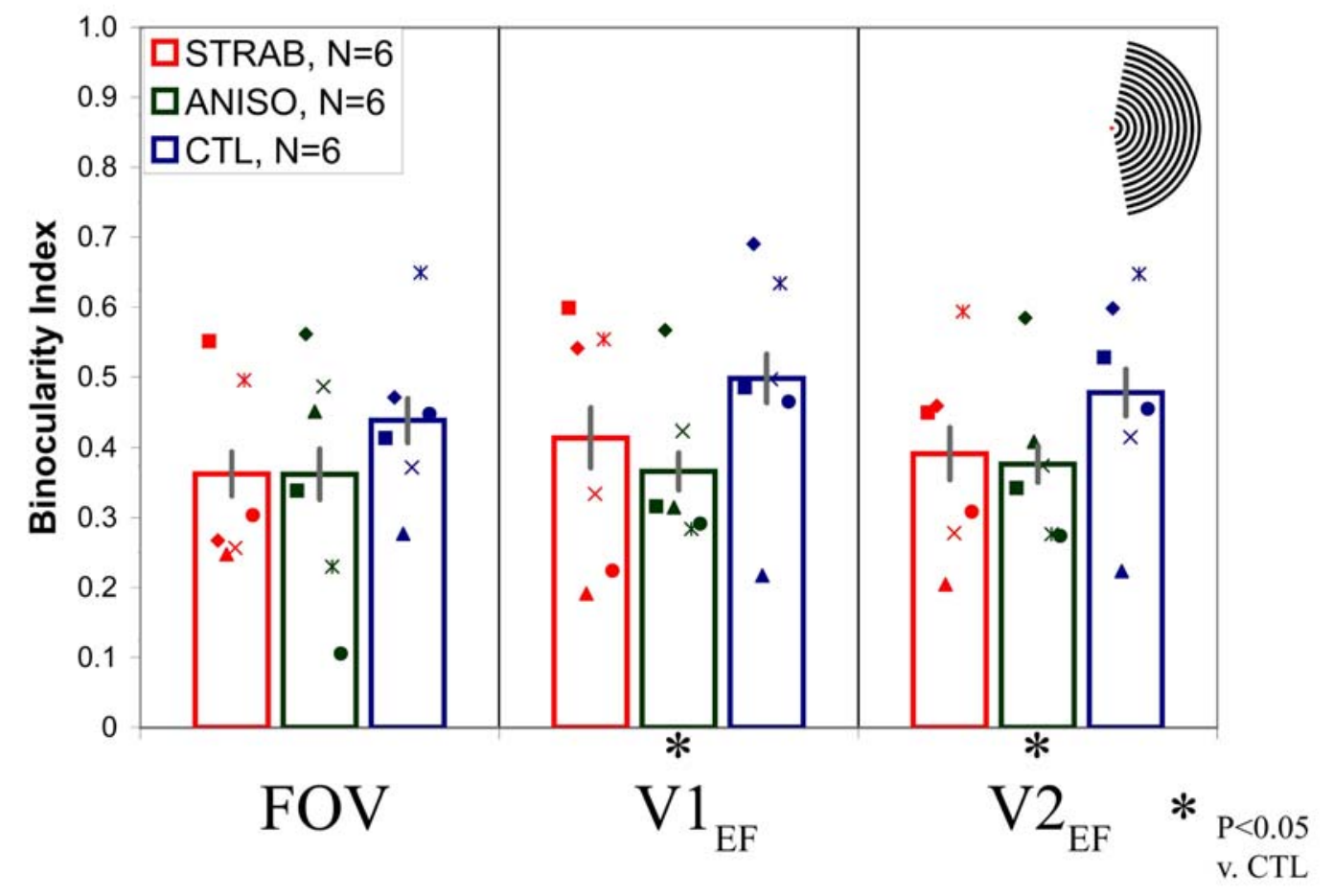

FIgURE 4-5. BINOCULARITY InDEX BY GROUP AND ROI. Mean BI is depressed in all ROIs for strabismic and anisometropic amblyopes. Labeling conventions are the same as in Fig. 4-3. 


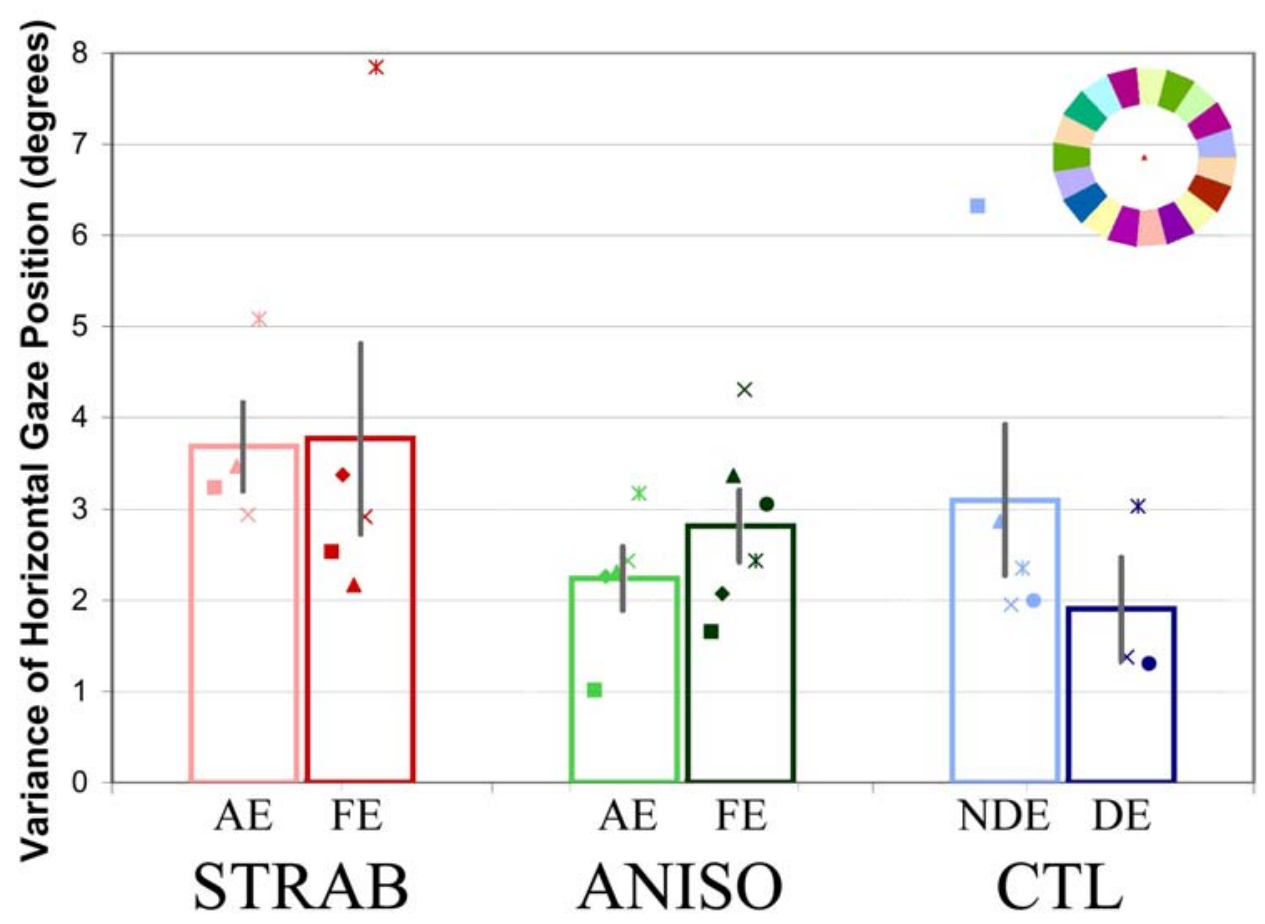

FIgURE 4-6. FiXATION STABILITY FOR EXPERIMENT 2. No significant differences of fixation stability are seen for either intergroup or interocular comparisons. Calibration error resulted in lack of eye tracking data in two strabismics (S2, S6), one anisometrope (A6), and one control (C2). Labeling conventions are the same as in Fig. 4-3. 


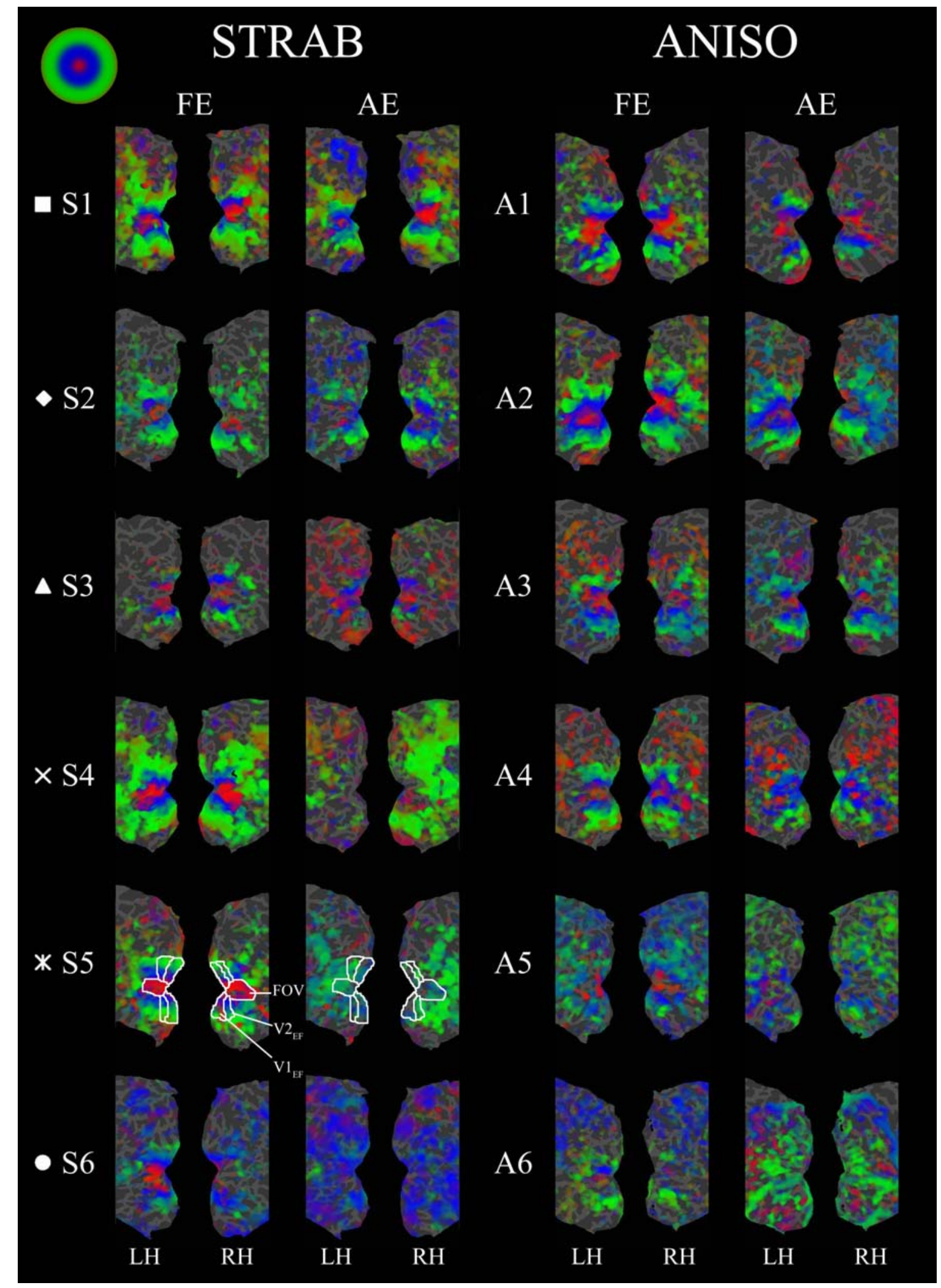


FIgURE 4-7. ECCENTRICITY MAPS FROM AMBLYOPIC PATIENTS. Eccentricity maps are shown on left and right hemisphere flattened occipital poles for fellow and amblyopic eyes of twelve subjects. These maps are arranged in order from most (top) to least (bottom) normal in appearance. Red, blue, and green indicate the central 0-2.5 deg, 2.5-8.5 deg, and 8.5-15 deg eccentricity, respectively (see key in upper left corner). Note the trend for more organized arrangement of colors in the fellow eyes vs. the disorganized patterns in the amblyopic eyes, especially within the ROIs. White outlines show the location of FOV, $\mathrm{V} 1_{\mathrm{EF}}$, and $\mathrm{V} 2_{\mathrm{EF}}$ for strabismic subject S5. Light and dark gray regions indicate the unfolded gyri and sulci, respectively. 


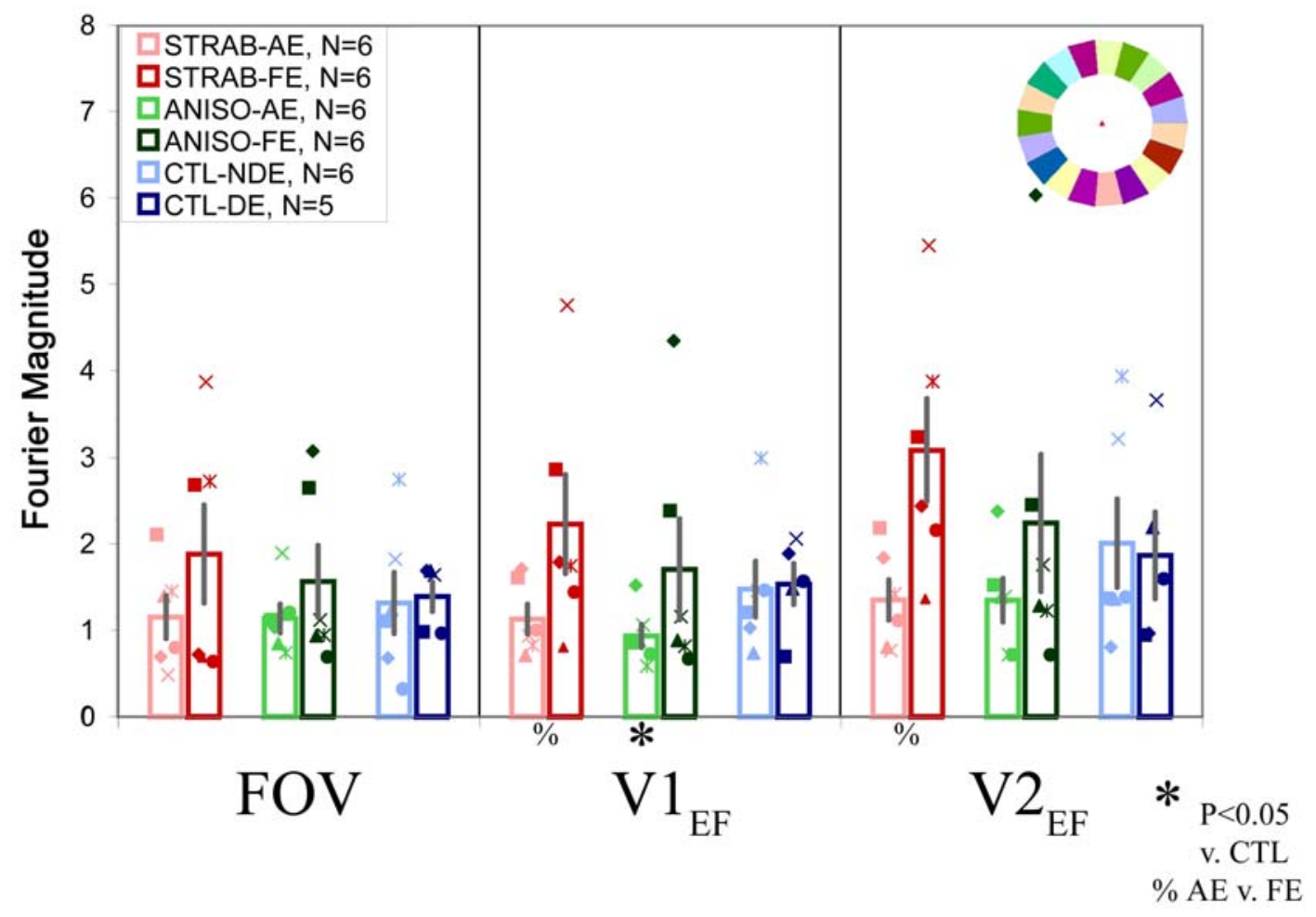

FIgURE 4-8. Fourier MAgNitUde FOR EXPERIMENT 2. Mean Fourier magnitude is depressed for every intergroup and interocular comparison. Percent symbols indicate significant interocular differences. Other labeling conventions are the same as in Fig. 4-3. 


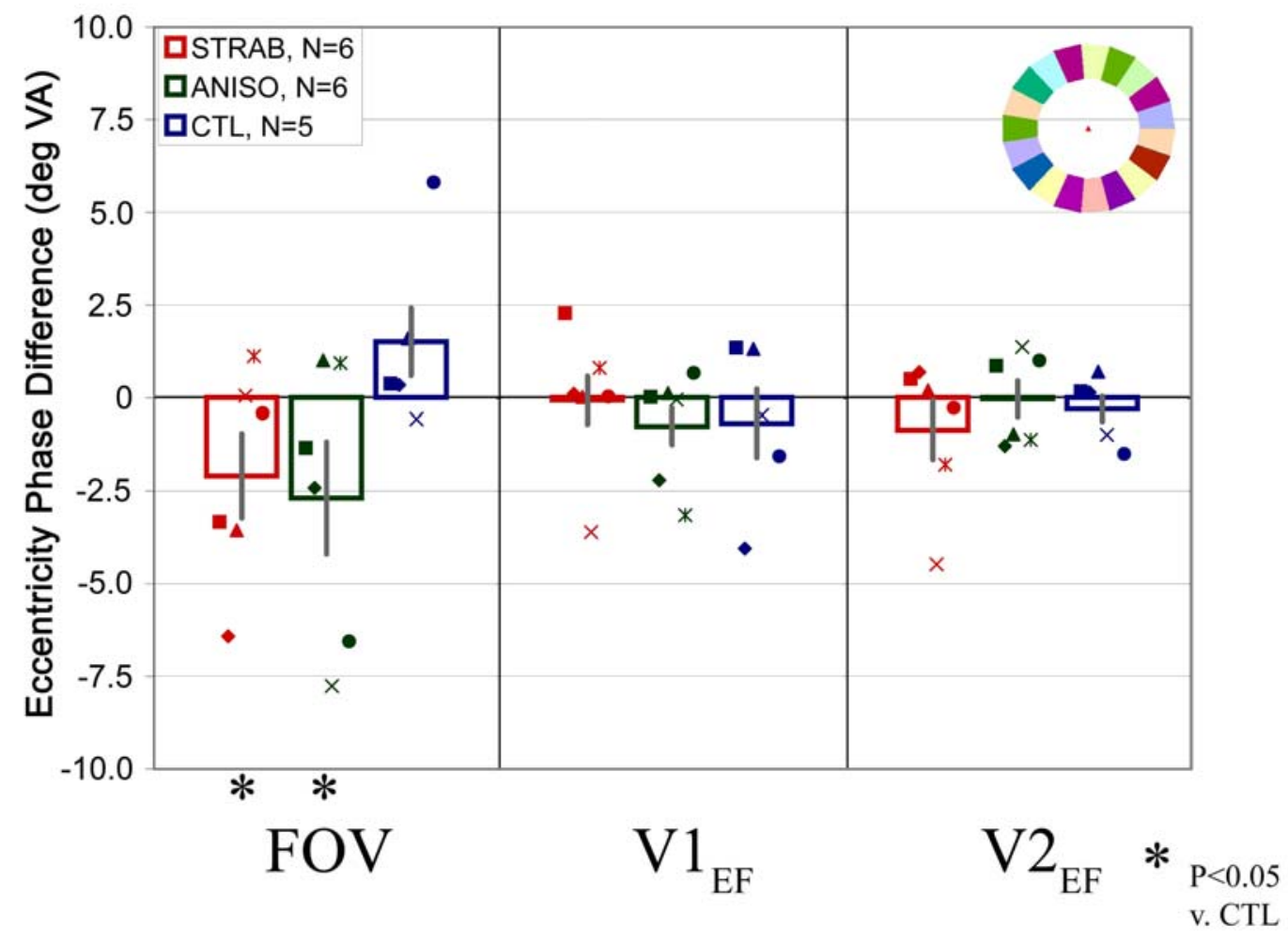

Figure 4-9. ECCENTRicity Phase DiffEREnCE. Mean interocular phase difference (FE - AE) was significantly more negative for both amblyopic groups in FOV. Labeling conventions are the same as in Fig. 4-3. 


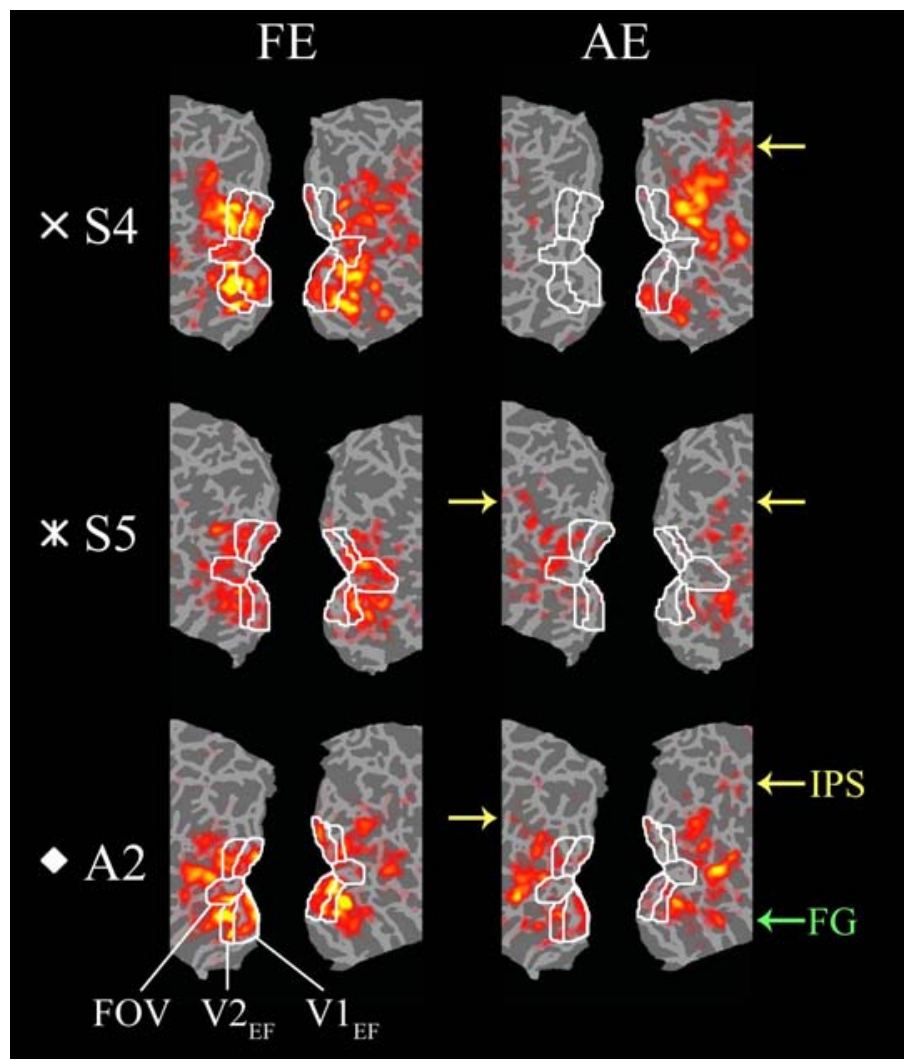

Figure 4-10. Parietal and Temporal Activation in Three Amblyopic SubJects. Increased activation was seen in the intraparietal sulcus (IPS, yellow arrows) and fusiform gyrus (FG, green arrow) for amblyopic eye stimulation in a subset of subjects. White outlines demonstrate the location of the foveal (FOV), $\mathrm{V} 1_{\mathrm{EF}}$, and $\mathrm{V} 2_{\mathrm{EF}}$ ROIs for these subjects. These results are summarized with Talairach coordinates in Table 4. 
A.
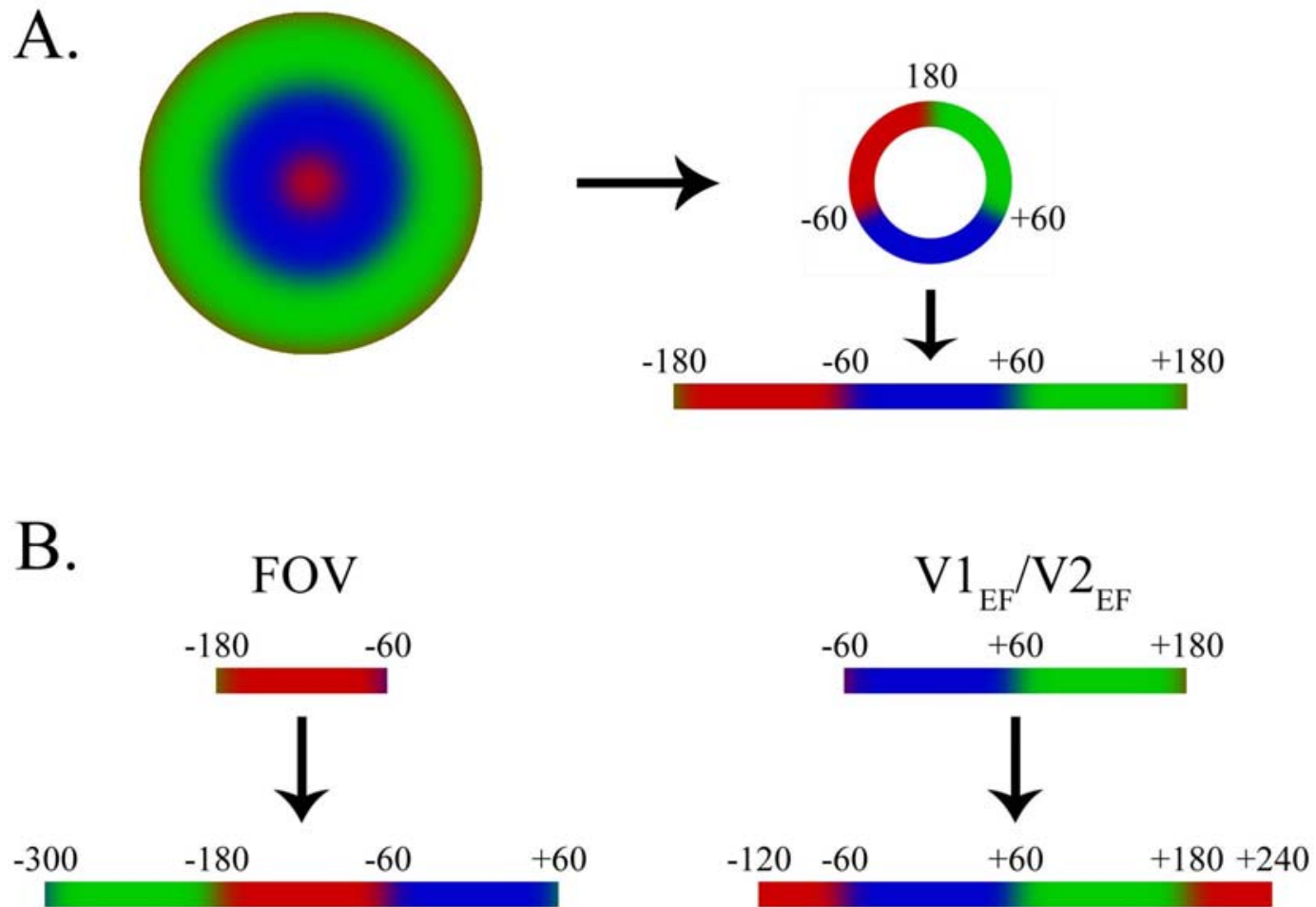

Figure 4-S1. ECCENTRICITY Phase CALCUlATION FOR ExPERIMENT 2. Mean eccentricity phases were calculated within each ROI (see text). A. Retinotopic location is assigned a phase and color value for eccentricity mapping. Phase is represented on a continuum from -180 to +180 degrees. Linearizing phase introduces a discontinuity, but the imprecision of the phase estimate results in ambiguity. B. Predicted phase values for the foveal (left) and V1/V2 (right) ROIs fall within a defined range, but values outside of this range are still found in the ROIs. Phase values are recentered around the predicted range in order to avoid systematic bias in mean phase calculation. 
A.
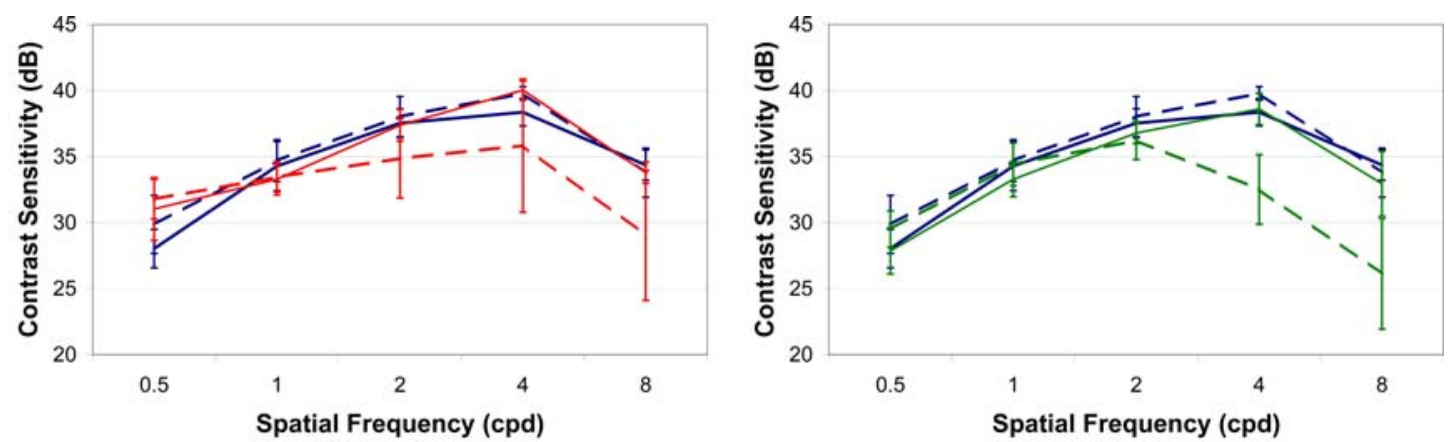

B.
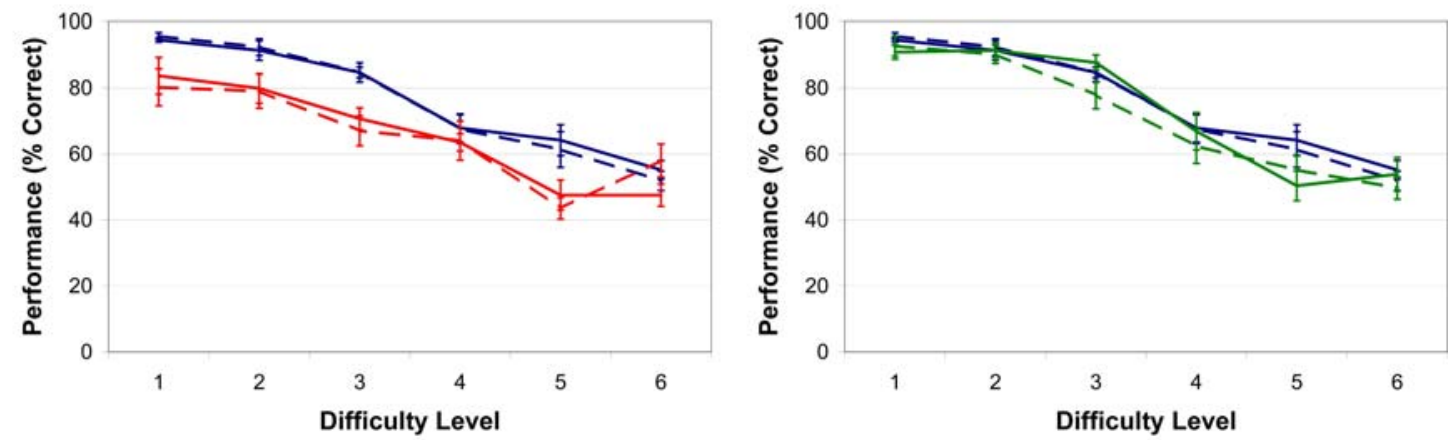

$-\cdot$ CTL-NDE —CTL-DE - -STRAB-AE — STRAB-FE

Figure 4-S2. Results from Psychophysics Testing. A. Contrast sensitivity functions for strabismic and anisometropic subjects show deficits in the higher spatial frequency range. B. Contour integration is impaired at all levels for both amblyopic and fellow eyes of strabismic subjects. 


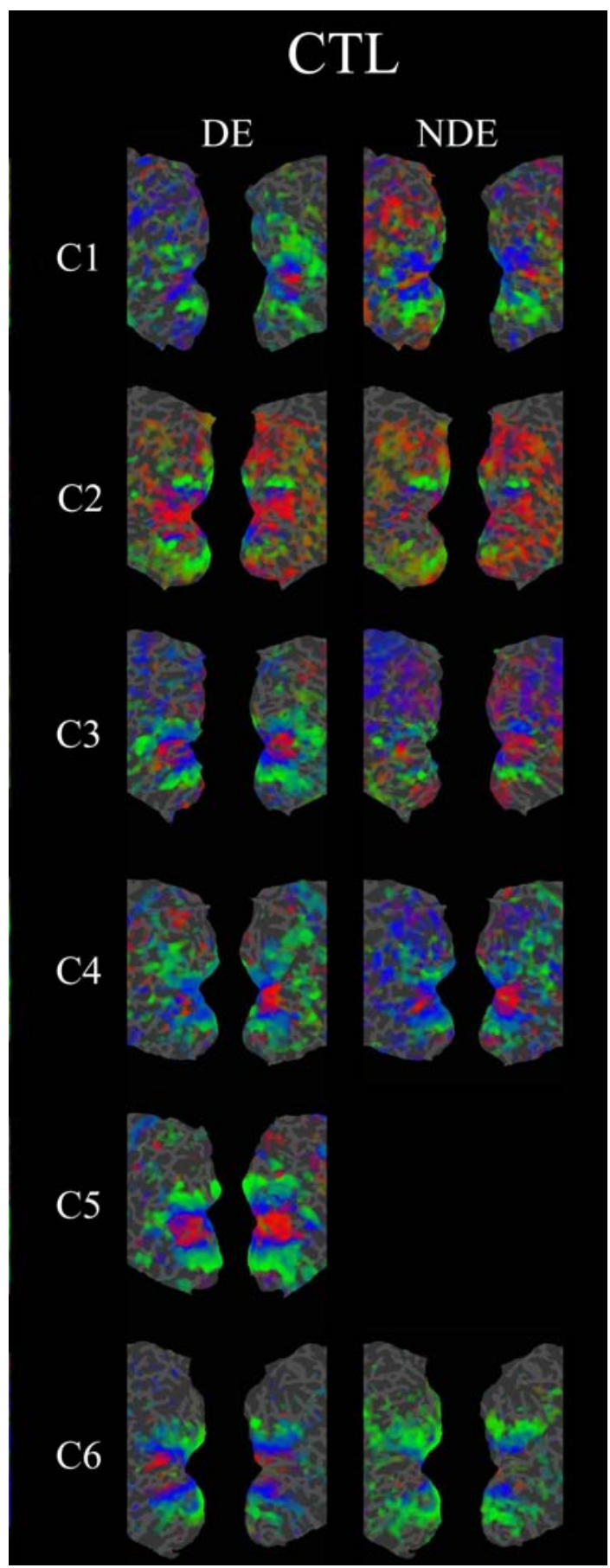

FIGURE 4-S3. ECCENTRICITY MAPS FROM CONTROL PATIENTS. Eccentricity maps are shown on left and right hemisphere flattened occipital poles for dominant and nondominant eyes of six subjects. These maps are arranged in order from most (top) to least (bottom) normal in appearance. Red, blue, and green indicate the central 0-2.5 deg, 2.5-8.5 deg, and 8.5-15 deg eccentricity, respectively. Light and dark gray regions indicate the unfolded gyri and sulci. 


\subsection{References}

Abrahamsson M, Sjostrand J (1988) Contrast sensitivity and acuity relationship in strabismic and anisometropic amblyopia. Br J Ophthalmol 72:44-49.

Algaze A, Roberts C, Leguire L, Schmalbrock P, Rogers G (2002) Functional magnetic resonance imaging as a tool for investigating amblyopia in the human visual cortex: a pilot study. J AAPOS 6:300-308.

Asper L, Crewther D, Crewther SG (2000a) Strabismic amblyopia. Part 2. Neural processing. Clin Exp Optom 83:200-211.

Asper L, Crewther D, Crewther SG (2000b) Strabismic amblyopia. Part 1. Psychophysics. Clin Exp Optom 83:49-58.

Baitch LW, Levi DM (1988) Evidence for nonlinear binocular interactions in human visual cortex. Vision Res 28:1139-1143.

Barnes GR, Hess RF, Dumoulin SO, Achtman RL, Pike GB (2001) The cortical deficit in humans with strabismic amblyopia. J Physiol 533:281-297.

Barrett BT, Bradley A, McGraw PV (2004) Understanding the neural basis of amblyopia. Neuroscientist 10:106-117.

Baseler HA, Brewer AA, Sharpe LT, Morland AB, Jagle H, Wandell BA (2002) Reorganization of human cortical maps caused by inherited photoreceptor abnormalities. Nat Neurosci 5:364-370.

Baseler HA, Morland AB, Wandell BA (1999) Topographic organization of human visual areas in the absence of input from primary cortex. J Neurosci 19:2619-2627.

Blatt GJ, Andersen RA, Stoner GR (1990) Visual receptive field organization and corticocortical connections of the lateral intraparietal area (area LIP) in the macaque. J Comp Neurol 299:421-445.

Bodis-Wollner I (1980) Detection of visual defects using the contrast sensitivity function. Int Ophthalmol Clin 20:135-153.

Bradley A, Freeman RD (1981) Contrast sensitivity in anisometropic amblyopia. Invest Ophthalmol Vis Sci 21:467-476.

Brainard DH (1997) The Psychophysics Toolbox. Spat Vis 10:433-436.

Braun J (1999) On the detection of salient contours. Spat Vis 12:211-225.

Campos E (1995) Amblyopia. Surv Ophthalmol 40:23-39. 
Cavada C (2001) The visual parietal areas in the macaque monkey: current structural knowledge and ignorance. Neuroimage 14:S21-S26.

Chan ST, Tang KW, Lam KC, Chan LK, Mendola JD, Kwong KK (2004) Neuroanatomy of adult strabismus: a voxel-based morphometric analysis of magnetic resonance structural scans. Neuroimage 22:986-994.

Chandna A, Pennefather PM, Kovacs I, Norcia AM (2001) Contour integration deficits in anisometropic amblyopia. Invest Ophthalmol Vis Sci 42:875-878.

Chelazzi L, Marzi CA, Panozzo G, Pasqualini N, Tassinari G, Tomazzoli L (1988) Hemiretinal differences in speed of light detection in esotropic amblyopes. Vision Res 28:95-104.

Chino YM, Kaas JH, Smith EL, III, Langston AL, Cheng H (1992) Rapid reorganization of cortical maps in adult cats following restricted deafferentation in retina. Vision Res 32:789796.

Chino YM, Smith EL, III, Kaas JH, Sasaki Y, Cheng H (1995) Receptive-field properties of deafferentated visual cortical neurons after topographic map reorganization in adult cats. J Neurosci 15:2417-2433.

Choi MY, Lee KM, Hwang JM, Choi DG, Lee DS, Park KH, Yu YS (2001) Comparison between anisometropic and strabismic amblyopia using functional magnetic resonance imaging. Br J Ophthalmol 85:1052-1056.

Ciuffreda KJ, Fisher SK (1987) Impairment of contrast discrimination in amblyopic eyes. Ophthalmic Physiol Opt 7:461-467.

Ciuffreda KJ, Kenyon RV, Stark L (1980) Increased drift in amblyopic eyes. Br J Ophthalmol 64:7-14.

Conner IP, Sharma S, Lemieux SK, Mendola JD (2004) Retinotopic organization in children measured with fMRI. J Vis 4:509-523.

Corbetta M, Shulman GL (2002) Control of goal-directed and stimulus-driven attention in the brain. Nat Rev Neurosci 3:201-215.

Cox RW, Jesmanowicz A (1999) Real-time 3D image registration for functional MRI. Magn Reson Med 42:1014-1018.

Crawford ML, Harwerth RS (2004) Ocular dominance column width and contrast sensitivity in monkeys reared with strabismus or anisometropia. Invest Ophthalmol Vis Sci 45:3036-3042.

Cynader MS (1982) Competitive neuronal interactions underlying amblyopia. Hum Neurobiol $1: 35-39$.

Dale AM, Fischl B, Sereno MI (1999) Cortical surface-based analysis. I. Segmentation and surface reconstruction. Neuroimage 9:179-194. 
Dale AM, Sereno MI (1993) Improved localization of cortical activity by combining EEG and MEG with MRI cortical surface reconstruction. J Cogn Neurosci 5:162-176.

Daw NW (1998) Critical periods and amblyopia. Arch Ophthalmol 116:502-505.

Demer JL, Grafton S, Marg E, Mazziotta JC, Nuwer M (1997) Positron-emission tomographic study of human amblyopia with use of defined visual stimuli. J AAPOS 1:158-171.

Demer JL, von Noorden GK, Volkow ND, Gould KL (1988) Imaging of cerebral blood flow and metabolism in amblyopia by positron emission tomography. Am J Ophthalmol 105:337-347.

Engel SA, Glover GH, Wandell BA (1997) Retinotopic organization in human visual cortex and the spatial precision of functional MRI. Cereb Cortex 7:181-192.

Eysel UT, Schweigart G (1999) Increased receptive field size in the surround of chronic lesions in the adult cat visual cortex. Cereb Cortex 9:101-109.

Faillenot I, Sunaert S, Van Hecke P, Orban GA (2001) Orientation discrimination of objects and gratings compared: an fMRI study. Eur J Neurosci 13:585-596.

Field DJ, Hayes A, Hess RF (1993) Contour integration by the human visual system: evidence for a local "association field". Vision Res 33:173-193.

Fischl B, Liu A, Dale AM (2001) Automated manifold surgery: constructing geometrically accurate and topologically correct models of the human cerebral cortex. IEEE Trans Med Imaging 20:70-80.

Fischl B, Sereno MI, Dale AM (1999) Cortical surface-based analysis. II: Inflation, flattening, and a surface-based coordinate system. Neuroimage 9:195-207.

Glover G (1999) Simple analytic spiral K-space algorithm. Magn Reson Med 42:412-415.

Goodyear BG, Nicolle DA, Humphrey GK, Menon RS (2000) BOLD fMRI response of early visual areas to perceived contrast in human amblyopia. J Neurophysiol 84:1907-1913.

Goodyear BG, Nicolle DA, Menon RS (2002) High resolution fMRI of ocular dominance columns within the visual cortex of human amblyopes. Strabismus 10:129-136.

Harrad RA, Hess RF (1992) Binocular integration of contrast information in amblyopia. Vision Res 32:2135-2150.

Hess RF (2001) Amblyopia: sight unseen. Clin Exp Optom 84:321-336.

Hess RF, Howell ER (1977) The threshold contrast sensitivity function in strabismic amblyopia: evidence for a two type classification. Vision Res 17:1049-1055.

Hess RF, Pointer JS (1985) Differences in the neural basis of human amblyopia: the distribution of the anomaly across the visual field. Vision Res 25:1577-1594. 
Hillis A, Flynn JT, Hawkins BS (1983) The evolving concept of amblyopia: a challenge to epidemiologists. Am J Epidemiol 118:192-205.

Horton JC, Hocking DR (1996) Pattern of ocular dominance columns in human striate cortex in strabismic amblyopia. Vis Neurosci 13:787-795.

Horton JC, Stryker MP (1993) Amblyopia induced by anisometropia without shrinkage of ocular dominance columns in human striate cortex. Proc Natl Acad Sci U S A 90:5494-5498.

Hubel DH, Wiesel TN (1962) Receptive fields, binocular interaction and functional architecture in the cat's visual cortex. J Physiol 160:106-154.

Hubel DH, Wiesel TN, LeVay S (1976) Functional architecture of area 17 in normal and monocularly deprived macaque monkeys. Cold Spring Harb Symp Quant Biol 40:581-589.

Imamura K, Richter H, Fischer H, Lennerstrand G, Franzen O, Rydberg A, Andersson J, Schneider H, Onoe H, Watanabe Y, Langstrom B (1997) Reduced activity in the extrastriate visual cortex of individuals with strabismic amblyopia. Neurosci Lett 225:173-176.

Ing MR, Pang SW (1974) The racial distribution of strabismus. A statistical study. Hawaii Med J 33:22-23.

Kiorpes L, Boothe RG (1981) Naturally occurring strabismus in monkeys (Macaca nemestrina). Invest Ophthalmol Vis Sci 20:257-263.

Kovacs I, Julesz B (1993) A closed curve is much more than an incomplete one: effect of closure in figure-ground segmentation. Proc Natl Acad Sci U S A 90:7495-7497.

Kovacs I, Kozma P, Feher A, Benedek G (1999) Late maturation of visual spatial integration in humans. Proc Natl Acad Sci U S A 96:12204-12209.

Kovacs I, Polat U, Pennefather PM, Chandna A, Norcia AM (2000) A new test of contour integration deficits in patients with a history of disrupted binocular experience during visual development. Vision Res 40:1775-1783.

Kwong KK, Belliveau JW, Chester DA, Goldberg IE, Weiskoff RM, Poncelet BP, Kennedy DN, Hoppel BE, Cohen MS, Turner R, Rosen B, Brady TJ (1992) Dynamic magnetic resonce imaging of human brain activity during primary sensory stimulation. Proc Natl Acad Sci U S A 89:5675-5679.

Lancaster JL, Woldorff MG, Parsons LM, Liotti M, Freitas CS, Rainey L, Kochunov PV, Nickerson D, Mikiten SA, Fox PT (2000) Automated Talairach atlas labels for functional brain mapping. Hum Brain Mapp 10:120-131.

Lee KM, Lee SH, Kim NY, Kim CY, Sohn JW, Choi MY, Gyu CD, Hwang JM, Ho PK, Lee DS, Suk YY, Hyun CK (2001) Binocularity and spatial frequency dependence of calcarine activation in two types of amblyopia. Neurosci Res 40:147-153. 
Lerner Y, Pianka P, Azmon B, Leiba H, Stolovitch C, Loewenstein A, Harel M, Hendler T, Malach R (2003) Area-specific amblyopic effects in human occipitotemporal object representations. Neuron 40:1023-1029.

Levi DM, Hariharan S, Klein SA (2002) Suppressive and facilitatory spatial interactions in amblyopic vision. Vision Res 42:1379-1394.

Levi DM, Harwerth RS, Smith EL (1980) Binocular interactions in normal and anomalous binocular vision. Doc Ophthalmol 49:303-324.

Levi DM, Klein S (1982) Hyperacuity and amblyopia. Nature 298:268-270.

Levi DM, Klein SA (1985) Vernier acuity, crowding and amblyopia. Vision Res 25:979-991.

Levi DM, Polat U (1996) Neural plasticity in adults with amblyopia. Proc Natl Acad Sci U S A 93:6830-6834.

Lewis JW, Van Essen DC (2000) Corticocortical connections of visual, sensorimotor, and multimodal processing areas in the parietal lobe of the macaque monkey. J Comp Neurol 428:112-137.

Liu GT, Miki A, Francis E, Quinn GE, Modestino EJ, Bonhomme GR, Haselgrove JC (2004) Eye dominance in visual cortex in amblyopia using functional magnetic resonance imaging. J AAPOS 8:184-186.

Malach R, Reppas JB, Benson RR, Kwong KK, Jiang H, Kennedy WA, Ledden PJ, Brady TJ, Rosen BR, Tootell RB (1995) Object-related activity revealed by functional magnetic resonance imaging in human occipital cortex. Proc Natl Acad Sci U S A 92:8135-8139.

Mayer DL, Fulton AB (1985) Preferential looking grating acuities of infants at risk of amblyopia. Trans Ophthalmol Soc U K 104 ( Pt 8):903-911.

Mayer DL, Fulton AB, Rodier D (1984) Grating and recognition acuities of pediatric patients. Ophthalmology 91:947-953.

McKee SP, Levi DM, Movshon JA (2003) The pattern of visual deficits in amblyopia. J Vis 3:380-405.

Mendola JD, Conner IP, Roy A, Chan ST, Schwartz TL, Odom JV, Kwong KK (2005) Voxelbased analysis of MRI detects abnormal visual cortex in children and adults with amblyopia. Human Brain Mapping (In Press).

Mendola JD, Dale AM, Fischl B, Liu AK, Tootell RB (1999) The representation of illusory and real contours in human cortical visual areas revealed by functional magnetic resonance imaging. J Neurosci 19:8560-8572. 
Mizoguchi S, Suzuki Y, Kiyosawa M, Mochizuki M, Ishii K (2005) Differential activation of cerebral blood flow by stimulating amblyopic and fellow eye. Graefes Arch Clin Exp Ophthalmol.

Movshon JA, Eggers HM, Gizzi MS, Hendrickson AE, Kiorpes L, Boothe RG (1987) Effects of early unilateral blur on the macaque's visual system. III. Physiological observations. J Neurosci 7:1340-1351.

Ogawa S, Tank W, Menon RS, Ellermann JM, Kim SG, Merkle H, Ugurbil K (1992) Intrinsic signal changes accompanying sensory stimulation: functional brain mapping with magnetic resonce imaging. Proc Natl Acad Sci U S A 89:5951-5955.

Pelli DG (1997) The VideoToolbox software for visual psychophysics: transforming numbers into movies. Spat Vis 10:437-442.

Pennefather PM, Chandna A, Kovacs I, Polat U, Norcia AM (1999) Contour detection threshold: repeatability and learning with 'contour cards'. Spat Vis 12:257-266.

Polat U, Ma-Naim T, Belkin M, Sagi D (2004) Improving vision in adult amblyopia by perceptual learning. Proc Natl Acad Sci U S A 101:6692-6697.

Rosa MG, Schmid LM, Calford MB (1995) Responsiveness of cat area 17 after monocular inactivation: limitation of topographic plasticity in adult cortex. J Physiol 482 ( Pt 3):589608.

Schor CM, Levi DM (1980) Disturbances of small-field horizontal and vertical optokinetic nystagmus in amblyopia. Invest Ophthalmol Vis Sci 19:668-683.

Sereno MI, Dale AM, Reppas JB, Kwong KK, Belliveau JW, Brady TJ, Rosen BR, Tootell RB (1995) Borders of multiple visual areas in humans revealed by functional magnetic resonance imaging. Science 268:889-893.

Sereno MI, McDonald CT, Allman JM (1994) Analysis of retinotopic maps in extrastriate cortex. Cereb Cortex 4:601-620.

Sireteanu R (1982) Human amblyopia: consequence of chronic interocular suppression. Hum Neurobiol 1:31-33.

Sireteanu R, Fronius M (1981) Naso-temporal asymmetries in human amblyopia consequence of long-term interocular suppression. Vision Res 21:1055-1063.

Sireteanu R, Fronius M (1990) Human amblyopia: structure of the visual field. Exp Brain Res 79:603-614.

Smith EL, III, Chino YM, Ni J, Cheng H, Crawford ML, Harwerth RS (1997) Residual binocular interactions in the striate cortex of monkeys reared with abnormal binocular vision. $\mathrm{J}$ Neurophysiol 78:1353-1362. 
Stuart JA, Burian HM (1962) A study of separation difficulty. Its relationship to visual acuity in normal and amblyopic eyes. Am J Ophthalmol 53:471-477.

Talairach J, Tournoux P (1988) Co-planar stereotaxic atlas of the human brain. New York: Thieme.

Thomas J (1978) Normal and amblyopic contrast sensitivity function in central and peripheral retinas. Invest Ophthalmol Vis Sci 17:746-753.

Tootell RB, Mendola JD, Hadjikhani NK, Liu AK, Dale AM (1998) The representation of the ipsilateral visual field in human cerebral cortex. Proc Natl Acad Sci U S A 95:818-824.

Tsutsui J, Fukai S (1980) Studies on visual evoked cortical response by liquid crystal phase difference haploscope: amblyopia and/or strabismus. J Pediatr Ophthalmol Strabismus 17:185-190.

Tychsen L, Lisberger SG (1986) Maldevelopment of visual motion processing in humans who had strabismus with onset in infancy. J Neurosci 6:2495-2508.

von Noorden GK, Campos EC (2001) Binocular vision and ocular motility: Theory and management of strabismus. St. Louis: Mosby.

Westall CA, Aslin RN (1984) Fixational eye movements and autokinesis in amblyopes. Ophthalmic Physiol Opt 4:333-337.

Wiesel TN, Hubel DH (1963) Single-cell responses in striate cortex of kittens deprived of vision in one eye. J Neurophysiol 26:1003-1017.

Zepeda A, Vaca L, Arias C, Sengpiel F (2003) Reorganization of visual cortical maps after focal ischemic lesions. J Cereb Blood Flow Metab 23:811-820. 


\section{CHAPTER 5: Monocular Representation in Visual Cortex of Amblyopic Children Measured with fMRI}




\subsection{Abstract}

Amblyopia is a developmental disorder that is known to result in abnormalities in primary visual cortex, although characterization of its neural substrates, especially in children, remains unexplored. This fMRI study directly compared the BOLD signals produced by monocular stimulation in nine children ( 3 esotropic strabismics, 3 anisometropes, and 3 controls). Measures were made in two cortical regions-of-interest (ROIs), corresponding to the representation of the nonamblyopic eye's fovea at the occipital pole and to an anatomically defined region of calcarine cortex, excluding the foveal ROI. Fixation stability was monitored in six subjects and was not found to correlate with BOLD signal strength. Overall, results showed depressed fMRI signal magnitude for anisometropic eyes compared with fellow eyes in all ROIs. Strabismic subjects showed mildly depressed signal in both eyes compared with controls. Analysis of individual phase-encoded retinotopic maps obtained from amblyopic eyes revealed fewer vertices in the occipital pole activated by foveal stimuli, consistent with known psychophysical losses and previous results obtained in adults. These losses were more profound for the anisometropic subjects. We conclude that the development of abnormal visual field organization, as seen in adults with amblyopia, has occurred in children ages 10 to 14 . 


\subsection{Introduction}

Visual experience is critical for normal brain development. For over 40 years, early visual deprivation has been recognized as a cause of subsequently measured abnormalities in visual cortex (Wiesel and Hubel, 1963). The time course of deprivation is important in directing the pattern of deficits, since different visual functions are acquired at varying times and rates throughout development. The time windows for the normal maturation, or pathological disruption, of visual functions have been termed sensitive periods (Harwerth et al., 1986). Most sensitive periods for visual development begin in infancy, but plasticity in response to experience or insult continues for various lengths of time throughout childhood, depending on the visual function (Lewis and Maurer, 2005). Visual functions with neural substrates at higher levels of information processing tend to have more prolonged sensitive periods (Daw, 1994).

In general, the order of maturation has been shown to progress hierarchically, with simpler characteristics of vision developing before more advanced visual abilities. For example, orientation discrimination, an important early function, is already present at birth but continues refinement throughout postnatal development (Slater et al., 1988; Chapman et al., 1999; White et al., 2001). Development of grating acuity follows, rapidly improving from approximately 40 times worse than adults in the newborn to 8 times worse by six months, and reaching adult-like levels around 4 to 6 years of age (Mayer and Dobson, 1982; Maurer and Lewis, 2001). Spatial contrast sensitivity matures in parallel with acuity, improving to adult-like levels between 6 and 9 years of age (Bradley and Freeman, 1982; Ellemberg et al., 1999; Adams and Courage, 2002). Finally, stereopsis, a binocular visual function, relies upon the prior establishment of adequate monocular acuity and motor control of vergence eye movements (Thorn et al., 1994). Therefore, stereopsis does not appear until between 3 to 4 months of age, but then quickly matures to adultlike levels (relative to acuity) by about 6 months of age (Birch et al., 1982; Schor, 1985). Other visuo-spatial integration functions continue to mature well into the second decade (Kovacs et al., 1999; Skoczenski and Norcia, 2002). Nevertheless, available imaging techniques have demonstrated adult-like neural organization in much of visual cortex in children between 10 to 12 years of age (Conner et al., 2004).

Amblyopia (i.e., "lazy eye") is the most common disorder of visual development in humans, affecting $1-3 \%$ of the population. It is clinically defined as reduced visual acuity not 
due to eye disease and not correctable by refractive lenses. It is associated with an interruption in early childhood of the experience-dependent maturation of visual cortex, usually by visual deprivation, strabismus, or anisometropia. Deprivation by congenital cataract has the most severe impact, but is comparatively rare (Birch and Stager, 1996; Maurer et al., 1999). Strabismus (misalignment of the eyes) and anisometropia (unequal interocular refractive error) are far more common during early visual development, and are likely to lead to amblyopia if untreated within the first 1 to 2 years of life (Atkinson et al., 1982; Birch and Stager, 1985). In fact, any impediment to normal pattern vision arising before the end of the first decade can lead to milder amblyopic deficits, indicating that cortical plasticity remains until at least this age (Vaegan and Taylor, 1979). However, successful treatment of amblyopia through patching the good eye diminishes with increasing age, with the best results achieved before 5 to 7 years (Ellemberg et al., 2000; Magnusson et al., 2002).

Earlier investigations of human amblyopia relied upon careful psychophysical testing in adults, consistently finding impaired visual acuity and contrast sensitivity in amblyopic eyes (Ciuffreda and Fisher, 1987; Abrahamsson and Sjostrand, 1988; Asper et al., 2000). Similar results for visual acuity and contrast sensitivity have been found in amblyopic children as well (Howell et al., 1983; Rogers et al., 1987; Ellemberg et al., 2000). In adults it has been shown that these deficits vary across the visual field, affecting central more than peripheral vision (Thomas, 1978; Sireteanu and Fronius, 1990). In addition, the specific pattern of visual impairments has been suggested to depend on clinical subtype, with strabismic but not anisometropic adults showing Snellen and vernier acuity impairments which are more severe than their grating acuity deficits (Levi and Klein, 1985; Birch and Swanson, 2000). Interestingly, strabismic amblyopes also perform more poorly than anisometropes on positional acuity, contour integration, and spatial localization tasks (Levi et al., 1999; Kovacs et al., 2000; Sharma et al., 2000). Spatial localization deficits have also recently been confirmed in a group of children with strabismic amblyopia (mean age $=7$ years) (Fronius et al., 2004). Nevertheless, the neural substrates for these deficits remain unclear.

Although these behavioral studies of human amblyopia have provided vital information for guiding clinical decisions, studies using animal models are also quite useful because they present a more controlled opportunity for investigating the neural basis of amblyopia (Mitchell and MacKinnon, 2002). The visual deficits seen in adult monkeys with induced amblyopia are 
similar to the acuity, contrast sensitivity, and stereopsis losses seen in humans (Harwerth et al., 1983; Smith, III et al., 1985; Kiorpes et al., 1987). Electrophysiology in the primary visual cortex (V1) of adult amblyopic monkeys has shown impaired contrast sensitivity and binocularity at the level of single neurons (Kiorpes et al., 1998). However, these techniques have not yet been applied to young animals still within the sensitive periods of visual development.

Fewer techniques for measuring neural activity in humans are available, but data are consistent with the information from animal models. The pattern reversal visual evoked potential (VEP) is commonly used to objectively assess acuity and contrast sensitivity in children otherwise unable to provide reliable feedback (Norcia et al., 1990; Skoczenski and Norcia, 1999). Specifically, in both adults and children with amblyopia, the P1 waveform of the VEP consistently has a longer latency and attenuated amplitude for the amblyopic eye compared with its fellow eye (Levi and Harwerth, 1978; Sokol, 1983; Henc-Petrinovic et al., 1993). Some differences between strabismic and anisometropic amblyopia have also been described, specifically that strabismics suffer a selective high spatial frequency deficit while anisometropes are impaired across the frequency range (Campos et al., 1984). Despite the usefulness of this data, these techniques are unable to provide detailed information for spatially localizing the source of the signal deficit within visual cortex.

In contrast, neuroimaging tools like positron-emission tomography (PET) and functional magnetic resonance imaging (fMRI) are able to localize signals within the brain, and have recently begun providing additional physiologic data from adults with amblyopia. PET has shown reduced cerebral blood flow and glucose metabolism in V1 and extrastriate cortex for amblyopic eye viewing (Demer et al., 1997; Imamura et al., 1997; Mizoguchi et al., 2005), while fMRI has been used to quantify reduced activation extent (Goodyear et al., 2000; Algaze et al., 2002) and signal magnitude in occipital cortex (Barnes et al., 2001; Liu et al., 2004). One recent fMRI study has extended these findings, showing abnormal cortical representation of visual space through the amblyopic eye of adult strabismic and anisometropic amblyopes (I. Conner, J. Odom, T. Schwartz, and J. Mendola, submitted). In these patients, visual field remapping is seen in early visual cortical areas for the amblyopic eye, such that occipital regions which map the foveal visual field of the fellow eye respond instead to parafoveal simulation when driven by the amblyopic eye. In addition, we also found abnormal activation of regions of parietal and 
temporal cortex for amblyopic eye viewing, suggesting atypical higher-order visual processing. These regionally specific abnormalities suggest that the visual field representation for the amblyopic eye might be functionally remapped through abnormal visual development in childhood.

Despite the progress that has been made in neurologically characterizing amblyopic adults with these imaging techniques, children with amblyopia have been rarely studied. One recent anatomical study using MRI voxel-based morphometry (VBM) showed reduced gray matter volumes in visual cortex for both adults and children with amblyopia (Mendola et al., 2005). Compared with control children, amblyopic children had less gray matter in calcarine and paracalcarine cortex, the medial and lateral parieto-occipital junction, and ventral temporal cortex. Thus, these children might be expected to show fMRI abnormalities in these regions, similar to those documented in adults. To date, no functional imaging studies have focused exclusively on amblyopic children, although one fMRI study including both children and adults found reduced cortical binocularity for strabismics and high spatial frequency deficits for anisometropes (Lee et al., 2001). However, this study did not separate their subjects for analysis by age, instead reporting only subtype-related differences.

The present study used monocular stimulation methods, similar to those previously used in adults, to study visual cortex in children (10 to 14 years old) with strabismic and anisometropic amblyopia. First, we used contrast sensitivity and contour integration psychophysics to demonstrate that the visual abilities of our children were similar to amblyopic children in other behavioral studies, as well as our previous group of amblyopic adults. Then, we used standard retinotopic mapping fMRI techniques to measure the cortical representation of visual field eccentricity. We hypothesized that amblyopic children in this age range would show the pattern of reduced fMRI activity and shifted receptive fields as reported in adults. We found that our amblyopic children did display this pattern, consistent with the hypothesis. 


\subsection{Materials and Methods}

\subsubsection{Subjects}

We studied 9 pediatric volunteers aged 10 to 14 (6 female, 3 male). Three were control (CTL) subjects, three had previously been diagnosed with strabismic (STRAB) amblyopia, and three had previously been diagnosed with anisometropic (ANISO) amblyopia (Table 1). Subjects with other known or suspected neurological or psychiatric conditions were excluded. These subjects were recruited through referral from the WVU Eye Institute from surrounding regions of West Virginia, Pennsylvania, and Maryland. Informed assent and consent was obtained from all participants and their parents or guardians (IRB protocol \# 14788), in accordance with the Code of Ethics of the World Medical Association (Declaration of Helsinki).

Our subject groups were matched for mean age $(\mathrm{CTL}=13, \mathrm{STRAB}=11, \mathrm{ANISO}=12)$ and mean parents' years of education $(\mathrm{CTL}=15, \mathrm{STRAB}=13$, ANISO $=14)$. All were righthanded. All amblyopic subjects had a history of occlusion treatment, i.e., patching, during childhood, but the presence of visual impairment at the time of testing in most subjects demonstrates that the deficit was never completely reversed (Table 1).

All subjects completed a full ophthalmologic exam at the WVU Eye Institute to confirm their diagnosis. Diagnosis of anisometropic amblyopia was assigned on the basis of 1.) Interocular refractive difference of hyperopia $>=+1.0$ diopter, astigmatism $>=+1.0$ diopter, or myopia $>=-2.5$ diopters; or 2.) History of anisometropia but no history of strabismus or strabismus surgery. Diagnosis of strabismus was made on the basis of a history of strabismus or strabismus surgery, but no anisometropia (as defined above).

The direction and magnitude of strabismic deviation in our subjects was determined with cover-uncover, alternate cover, and prism testing. All of our strabismic subjects had a history of inward eye deviations (esotropia) rather than outward deviations (exotropia). Congenital esotropia and exotropia are both commonly associated with amblyopia, although it has previously been shown that esotropia has the greater prevalence in Caucasian populations (Ing and Pang, 1974). Latent deviations for our subjects ranged from 0-20 prism diopters (Table 1).

The ophthalmologic tests also included examination of the fundus with dilation, documentation of ductions and versions, autorefraction, and a sensory exam including Snellen visual acuity (Lombart Instrument, Norfolk, VA), contrast sensitivity (Lighthouse International, 
New York, NY), Worth 4-dot, stereoacuity (Titmus Optical, Inc., Petersburg, VA), and Ishihara color plates (Kanehara and Co., Ltd., Tokyo, Japan). The results for Snellen visual acuity, interocular refractive difference, and Titmus stereoacuity are shown (Table 1). The Titmus stereoacuity test was scored according to highest level of detectable horizontal disparity for the Wirt rings or for the Titmus fly. The crudest stereoacuity measurable with this test is 3500 arcsec, assigned for patients able to perceive disparity only in the Titmus fly illustration.

\subsubsection{Psychophysical Testing}

All psychophysical tests were administered separately for the amblyopic (AE) and fellow (FE) eyes using individual optical correction. Translucent plastic patches covered the nontesting eye. All tests included practice trials to ensure that the stimuli were visible and the task instructions were adequately understood.

\subsubsection{Contrast Sensitivity}

Stimuli were generated using the Psychophysics Toolbox (Brainard, 1997; Pelli, 1997) for MATLAB 5 in Macintosh OS 9, and were displayed on a calibrated Apple Studio Display LCD monitor at $57 \mathrm{~cm}$. For each trial, the subject viewed two temporally sequenced epochs identified by audible tone, containing either a vertical sinusoidal grating (subtending 6 degrees of visual angle, $200 \mathrm{~ms}$ duration) or an isoluminant gray screen. The entire trial lasted $2400 \mathrm{~ms}$ with an intertrial interval of $1800 \mathrm{~ms}$. The subject identified the grating epoch, using 2-AFC. An interactive staircase procedure (2-up, 1-down) was used to approach the contrast detection threshold (the $71 \%$ correct level) at each of five spatial frequencies $(0.5,1.0,2.0,4.0$, and 8.0 cycles per degree), terminating after seven reversals for each spatial frequency. The first three reversals used a step size of $6 \mathrm{~dB}$ to rapidly approach threshold, while the last four reversals used a $2 \mathrm{~dB}$ step size. The contrast threshold was taken as the geometric mean of the last four reversals.

\subsubsection{Contour Integration}

Stimuli were displayed on a Silicon Graphics CRT monitor (model GDM-5411). The task was to determine the orientation (i.e., pointing left or right) of a perceived egg-shaped 
contour made up of 15 small aligned Gabor patches embedded in a field of randomly oriented patches of identical contrast and spatial frequency (Field et al., 1993; Kovacs and Julesz, 1993; Kovacs et al., 1999; Pennefather et al., 1999; Braun, 1999; Chandna et al., 2001). This test was administered using the method of constant stimuli and a 2-AFC paradigm at six levels of increasing difficulty (available online at http://zeus.rutgers.edu/ ikovacs/S\&P contour.html). Task difficulty increased as a function of increasing orientation jitter of the Gabor patches along the contour.

\subsubsection{Cortical Surface Reconstruction}

Cortical surface reconstructions were generated from individual high-resolution anatomical images obtained in a General Electric 1.5 Tesla scan session prior to the fMRI experiments, using the freely available (http://www.nmr.mgh.harvard.edu/freesurfer) FreeSurfer software package (Dale and Sereno, 1993; Fischl et al., 1999; Dale et al., 1999; Fischl et al., 2001). Briefly, whole-head 3D Fast Spoiled Grass (FSPGR) gradient echo scans, optimized for contrast between gray and white matter, were collected for each subject. Specific parameters were: fast IR prep (prep time $=300 \mathrm{~ms}$ ), $\mathrm{TE}=1.9 \mathrm{~ms}$, flip angle $=20 \mathrm{deg}, \mathrm{FOV}=24 \mathrm{~cm}$, axial slices, $256 \times 256$ matrix, voxel resolution $=0.94 \times 0.94 \times 1.2 \mathrm{~mm}$.

The cortical surface representation was inflated and then flattened by introducing a series

of cuts to the 3-D surface to isolate the occipital pole. Using this technique, the primary relaxation cut corresponds to calcarine sulcus, approximately the horizontal meridian representation in V1 (Sereno et al., 1995). The resulting occipital patch was used for fMRI data display, but all data analysis was performed in native space.

\subsubsection{Functional Magnetic Resonance Imaging}

\subsubsection{General Methods}

Subjects were scanned in a General Electric 1.5 Tesla MR scanner using a visual surface coil (Nova Medical, Inc., Wilmington, MA), as has been previously described (Mendola et al., 1999; Conner et al., 2004). After a sagittal localizing scan, a T1-weighted inversion recovery sequence ( $(\mathrm{TR}=400 \mathrm{~ms}$ ) was used to acquire 20 interleaved oblique $4 \mathrm{~mm}$ slices with $0.86 \times 0.86$ $\mathrm{mm}$ in-plane resolution, oriented perpendicular to the calcarine sulcus, beginning at the occipital 
pole. These anatomical scans were later utilized to register functional data to the cortical surface model.

The next step was to acquire multiple functional scanning runs using the same slice prescription selected in the anatomical scans, but with $3.44 \times 3.44 \mathrm{~mm}$ in-plane resolution. Functional signals reflecting the blood oxygenation level dependent (BOLD) contrast were acquired using a spiral gradient echo sequence $(\mathrm{TE}=40 \mathrm{~ms}$, Flip Angle $=65 \mathrm{deg}, \mathrm{TR}=4000$ ms) (Kwong et al., 1992; Ogawa et al., 1992; Glover, 1999).

Head movement (within- and between- scans) was minimized by the use of foam packing and adhesive tape around the subject's head within the MR scanner. Eye movements were monitored using the Sensomotorics iView system in order to ensure fixation stability during the functional scans. The iView system was used to measure gaze position in the stimulated eye, and was calibrated using a nine-point display at a screen resolution of $832 \times 624$ pixels, subtending approximately 30 deg horizontal x 23 deg vertical of visual angle. Accurate calibration was not achieved in all subjects (see Results), but most subjects were able to have eye tracking throughout the experiment. The iView camera recorded at $60 \mathrm{~Hz}$.

During the MR imaging experiments, the visual stimuli were generated using the Psychophysics Toolbox and MATLAB 5 for Macintosh OS 9 on a PowerMac G4 computer with dual SVGA display drivers (output resolution $=832 \times 624$ pixels, 30 deg horizontal $\times 23 \mathrm{deg}$ vertical). The stimuli were displayed in the scanner using the Avotec SilentVision dichoptic projector. Subjects viewed the images with both eyes open by looking straight ahead into the eyepieces, which were placed approximately $1 \mathrm{~cm}$ in front of their eyes. Subjects used the eyepieces' built-in optical correction. For each functional scanning run, one eyepiece displayed the stimulus and fixation target while the other displayed an isoluminant gray screen. Left and right eye stimulation was alternated during each experiment.

A central fixation mark was present at all times for the fMRI experiments. Subjects were clearly instructed to maintain fixation on this mark throughout the functional scanning. The fixation target was a small arrowhead ( $0.5 \mathrm{deg})$ pointing in one of four directions (i.e., up, down, left, or right) which randomly changed direction every $4 \mathrm{~s}$. In order to aid fixation stability and maintain attention to the stimulus, subjects were given a task of monitoring and reporting the appearance of the fixation point. Subjects used a fiber-optic button pad with four buttons configured in a cross shape to report arrow orientation. 


\subsubsection{Retinotopic Mapping}

The cortical representation of retinotopic visual space was determined using a phaseencoded design in which the eccentricity domain of visual space was mapped (Engel et al., 1997). The stimulus consisted of a high-contrast, multi-colored checkerboard "expanding ring," which mapped eccentricity by starting from the center of the visual field and expanding outward (Fig. 5-1). Eccentricity stimuli traversed space with a logarithmically increasing rate, as has been used previously (Sereno et al., 1995; Conner et al., 2004). This allowed the stimulus to spend a greater proportion of the cycle in the foveal portion of the visual field, approximately accounting for decreasing cortical magnification from central vision to the periphery. Both stimuli also approximately compensated for the increasing receptive field size by increasing in size as they approach the periphery.

These phase-encoded stimuli used a 64 s cycle and completed eight cycles per scan, as in the previous study of adults with amblyopia. As a result, each functional scan took $512 \mathrm{~s} \mathrm{(8} \mathrm{min}$ $32 \mathrm{~s}$ ), collecting 128 time points per functional scan volume. Four scans of this type were administered in one session, two each eye. Paired expansion/contraction scans were used in order to cancel the effects of residual hemodynamic phase delays. The entire retinotopic mapping experiment typically lasted about 1 hour.

\subsubsection{Statistical Analysis}

The functional analysis was completed using the FS-FAST software tools freely available at ftp://ftp.nmr.mgh.harvard.edu/pub/flat/fmri-analysis. Before statistical analysis, raw MR images were first motion-corrected to the $64^{\text {th }}$ volume (i.e., the midpoint of 128 volumes) of the first run using an iterated linearized weighted least squares method through the FS-FAST implementation of the AFNI 3dvolreg algorithm (Cox and Jesmanowicz, 1999). In addition to motion-correcting, this algorithm also provides a sum-of-squares estimate of average head motion throughout the fMRI scanning session, which was compared between subject groups for both experiments. The MR volumes were subsequently intensity normalized using the average in-brain voxel intensity. The resulting data set was then used as input for the subsequent Fourier analysis. 
Fast Fourier transform analysis was conducted on the time series of each voxel to statistically correlate retinotopic stimulus location with visual cortical anatomy. This analysis rejected low frequencies due to head motion or baseline drift and extracted functional signals in the form of magnitude and phase relative to the stimulus cycle frequency. Signal magnitude reflects retinotopic specificity, which can be low due to either lack of visually induced response or equivalent response to all retinotopic locations. The phase component of the signal codes specific retinotopic location, but is also dependent upon individual hemodynamic delay. To standardize the effect of hemodynamics across subjects, individual maps were first optimized with respect to phase for each subject's fellow (or dominant) eye, and the resulting phase offset was subsequently applied to the amblyopic eye maps. This consisted of choosing a phase offset that optimally aligned the representation of central vision with the occipital pole, resulting in a normal map with a concentric red (foveal), blue (parafoveal), and green (peripheral) pattern. Thus, the fellow eye was established to serve as an internal control for each subject. The data was projected onto flattened representations of individual brains using a tricolor code of signal phase, i.e., visual field location (Sereno et al., 1994; Sereno et al., 1995).

In order to directly compare eccentricity stimulus magnitude patterns between eyes in individual subjects, we further plotted the significance of the F-statistic for each eye onto flattened occipital patches. These significance maps more accurately show the regional strength of cortical activation in eccentricity scans, independent of phase.

\subsubsection{Region-of-Interest Analysis}

Two sources of information were used to create regions-of-interest (ROIs) for quantitative analysis. First, the eccentricity mapping data from the fellow eye, or the right eye for controls, was used to define a functional ROI for the region of the occipital pole driven by the central 2.5 degrees of eccentric visual angle. This ROI, corresponding to the foveal (FOV) portion of the visual field, was hypothesized to possess abnormal retinotopic organization. For comparison, a separate anatomically defined region was created for the calcarine sulcus and its bounding gyri, extending anteriorly to the parieto-occipital sulcus and posteriorly to the occipital pole. A third ROI was created by subtracting the foveal ROI from the calcarine ROI. This 
resulted in a separate ROI corresponding to the extrafoveal (ExFOV) calcarine cortex (i.e., > 2.5 degrees eccentricity).

The FOV and ExFOV ROIs were used to extract the Fourier magnitude and Fourier phase of the BOLD signal at each vertex, for each eye and ROI. Phase values range from -180 to +180 , with negative values corresponding to foveal and positive values corresponding to peripheral field stimulation. In order to allow for noise in the phase estimation, correct for the phase discontinuity at the end of the eccentricity cycle (i.e., periphery wraps around to fovea), and avoid systematic bias in the mean phase estimation, the continuous circular phase scale was linearized and recentered to form a bracket around the predicted phase values for each ROI. For the foveal ROI, the predicted phase of all vertices is -180 to -60 (the first third of the stimulus cycle), so phase values in the ROI corresponding to the last third of the stimulus cycle ( +60 to +180 ) were assumed to represent offset noise in the phase estimation and shifted by -360 , to -300 to -180 . This results in an effective range of -300 to +60 for the foveal ROI. Similarly, for EF, the predicted phase of all vertices is -60 to +180 (the final two-thirds of the stimulus cycle), so phase values corresponding to the first sixth of the stimulus cycle (-180 to -120) were shifted by +360 , to +180 to +240 , resulting in an effective range of -120 to +240 . This recentering procedure allowed the use of linear descriptive statistics for comparing phases across subjects. Mean phase was calculated for each ROI and eye in each subject. Interocular phase differences were then calculated (FE - AE or OS - OD). Using this method, negative phase differences indicate a relative shift of amblyopic eye vertices toward later phase values (i.e., driven by more peripheral visual field locations) and vice versa. 


\subsection{Results}

\subsubsection{Psychophysics}

\subsubsection{Contrast Sensitivity}

Contrast sensitivity in the amblyopic eyes of both groups was depressed at higher spatial frequencies (i.e., 2, 4, and $8 \mathrm{cpd}$ ) compared with control eyes (Fig. 5-2 A). This result is consistent with the commonly reported contrast sensitivity deficit in amblyopia (Hess and Howell, 1977; Bodis-Wollner, 1980; Bradley and Freeman, 1981; McKee et al., 2003).

\subsubsection{Contour Integration}

For strabismics, both the amblyopic and fellow eyes were impaired at low difficulty levels (Fig. 5-2 B), consistent with reports of impairments at this task in both strabismic and fellow eyes, independent of acuity (Kovacs et al., 2000). In contrast, only amblyopic eyes of anisometropes were impaired at this task, as has been shown elsewhere (Chandna et al., 2001). Previous work has suggested that strabismic and anisometropic amblyopia can be distinguished based on differences in higher-order visual functions such as vernier acuity and contour integration (Levi and Klein, 1982; Levi and Klein, 1985), so greater impairments in strabismics were expected. Overall, these results show that our strabismic and anisometropic subject groups are comparable to previous studies.

\subsubsection{Retinotopic Mapping}

\subsubsection{Head Motion and Fixation Stability}

A major concern in any functional neuroimaging experiment is subject cooperation, but this is particularly true when studying children. Previous neuroimaging work has shown that head motion is a potential confound with children (Conner et al., 2004). In the setting of impaired vision, it was especially important to ensure that head movements were no greater in amblyopes than in controls. To address this concern, we averaged the translational distance of the head from its original position for each time point of each scan to calculate the mean translational distance across scans for each subject. We found that mean across-scan translational head motion was equivalent across groups $(\mathrm{CTL}=2.2 \mathrm{~mm}$, STRAB $=2.6 \mathrm{~mm}$, 
ANISO $=1.7 \mathrm{~mm}$ ). This showed that the amblyopic children did not move in the scanner more than the control children.

Next, we wanted to determine if there were differences in fixation behavior between the amblyopic and control subjects, as poor fixation could also confound the interpretation of any fMRI differences. To achieve this goal, we used the iView eye tracking system to measure ocular movements during each fMRI experiment. Mean horizontal fixation variance was chosen to assess fixation because it is more variable than vertical fixation in strabismus (Westall and Aslin, 1984). However, we were not able to collect fixation data from all subjects, primarily due to failures in the eye tracker calibration software. In these cases, it was important to also use the fixation task accuracy to assess fixation and attention. All subjects provided feedback for $>80 \%$ of these trials.

For the anisometropes, we were able to monitor fixation in two subjects. Data from these subjects, A1 and A3, closely matched two control subjects, C2 and C1 (Fig. 5-3 A), and was in the same range as fixation stability reported previously in adults. Interestingly, our subject with the poorest amblyopic eye acuity and largest interocular refractive difference, A3 (Table 1), also had quite poor fixation task accuracy for amblyopic eye viewing (Fig. 5-3 B). However, fixation stability from $\mathbf{A} \mathbf{3}$ was nearly identical to $\mathbf{C} \mathbf{1}$, so poor acuity rather than fixation stability seems to explain this subject's task results.

For the strabismics, we were unable to monitor fixation in any amblyopic eye. Given the aforementioned concern regarding the potential confound of poor fixation, this certainly hampers our interpretation of fMRI data from strabismics. However, we were able to collect task performance data from two of our strabismics, S2 and S3, which was indistinguishable from control subjects' data (Fig. 5-3 B). Although it would have been preferable to confirm fixation in these subjects, their high level of task performance demonstrates that they discriminated and attended to the target, making large errors of fixation stability unlikely. Nevertheless, experimental eye tracking remains the standard, and should continue to be attempted in future experiments.

\subsubsection{2 fMRI Activation Maps}

Phase-encoded maps of eccentricity are shown for strabismic and anisometropic subjects (Fig. 5-4). These individual maps of eccentricity are vertically arranged according to a 
qualitative assessment of departure from the normal pattern, with the more abnormal activation patterns placed toward the bottom. Normal eccentricity maps are comprised of a concentricallyorganized pattern of red for central vision, blue for parafovea/mid-periphery, and green for periphery. S1 and A1, for example, possess eccentricity maps resembling the normal pattern for their fellow eyes. In general, the amblyopic eyes' maps were always less normal in appearance. Many of the amblyopic eye maps were grossly abnormal, including a lack of concentric organization and a clearly diminished representation of foveal signals. These abnormalities usually occurred in concert with fellow eye maps which demonstrated a normal pattern of organization, but moderately diminished strength of signal.

Significance maps $(p<0.05)$ for the Fourier signal magnitude were also plotted for each subject, independent of phase information (Fig. 5-5). With the exception of the mild anisometropic subject A1, all amblyopes had decreased signal magnitudes for both eyes compared with controls. It is interesting to note here that $\mathbf{A} \mathbf{1}$ had the smallest interocular refractive difference as well as nearly normal stereoacuity, which is a sensitive marker of amblyopia when impaired (Table 1).

\subsubsection{ROI Analysis of Fourier Magnitude}

The results of ROI analysis in the calcarine cortex were consistent with the qualitative results described above. Mean signal was less for amblyopic compared with fellow eyes in both ROIs for anisometropic subjects. In contrast, signal magnitudes in strabismic ROIs were low but similar in both eyes (Fig. 5-6).

In a separate analysis, we examined whether Fourier magnitude varied as a function of either fixation stability or task accuracy. Since there were only three subjects in each group and a significant number of missing data for both fixation stability and accuracy, we performed a Pearson correlation with signal magnitude for each measure across all subjects and eyes. We did not find a significant correlation between signal magnitude and either fixation stability (FOV: $\mathrm{R}^{2}$ $=0.13, p=0.31$; ExFOV: $\left.\mathrm{R}^{2}=0.09, p=0.41\right)$ or task accuracy (FOV: $\mathrm{R}^{2}=0.07, p=0.40$; ExFOV: $\mathrm{R}^{2}=0.04, p=0.50$ ).

\subsubsection{ROI Analysis of Eccentricity Phase}


Strabismic and anisometropic amblyopes both had negative interocular phase differences in FOV (FE-AE, Fig. 5-7). A negative interocular phase difference indicates that, in this ROI, the amblyopic eye preferred eccentricities from more peripheral visual field locations, compared with the fellow eye. This result is consistent with the observation that both amblyopic groups demonstrated reduced cortical representations of foveal stimuli from their amblyopic compared with their fellow eyes (Fig. 5-4). These results parallel the findings in a previous retinotopic mapping study of adults with amblyopia (I. Conner, J. Odom, T. Schwartz, and J. Mendola, submitted). Unexpectedly, the strabismic children had a large positive interocular phase difference in ExFOV, indicating that the strabismic eye abnormally preferred less peripheral visual field locations in ExFOV, compared with the fellow eye. Thus, we found a shift in strabismics toward preference for parafoveal stimulation, at the expense of both the central and peripheral visual field representations. Some previous studies have shown some impairment of peripheral as well as central vision in strabismics (Thomas, 1978; Katz et al., 1984). However, further experiments are clearly needed to confirm these results. 


\subsection{Discussion}

We have demonstrated that visual cortex in the brains of children with amblyopia contains an abnormal representation of the visual field. This result is in agreement with our previous study of adults with amblyopia, in which we found decreased mapping of foveal field locations and enhanced representation of parafoveal locations for the amblyopic eye. This is one of the few known fMRI studies of amblyopia in children, with important implications for monitoring and treating this critical visual disorder.

\subsubsection{Psychophysics}

In order to show that our subject groups were comparable to those in other behavioral studies of amblyopia, we first conducted some limited psychophysical testing. In addition to the clinical assessment of vision, we also collected contrast sensitivity and contour integration data. We found acuity, contrast sensitivity, and contour integration deficits that were similar to those found in amblyopic adults (I. Conner, J. Odom, T. Schwartz, and J. Mendola, submitted).

Threshold contrast sensitivity is commonly used to characterize amblyopia in psychophysics studies. When contrast sensitivity has been studied across a range of ages in normal children, the various studies agree that that adult-like levels are reached in normal children sometime between six (Ellemberg et al., 1999) and eight or nine years of age (Bradley and Freeman, 1982; Adams and Courage, 2002). Since our subjects were all at least 10 years old, we expected and found adult-like contrast sensitivity for our controls. For children with amblyopia, prior work has shown an impairment of contrast sensitivity at high spatial frequencies, which persists even while acuity improves with patching therapy (Lundh and Lennerstrand, 1983; Rogers et al., 1987). We found similar results in our amblyopic children. Finally, the literature has suggested that strabismics and anisometropes may differ with respect to contrast sensitivity, with strabismics suffering more severe acuity losses relative to their contrast sensitivity deficits than anisometropes (Abrahamsson and Sjostrand, 1988). We do not see this trend in our data; however, this effect would likely require a larger sample to become evident.

It has been proposed that spatial contour integration continues its development in children with normal vision until at least midway through the second decade (Kovacs et al., 1999). Although the children we studied are nearing this age of maturity, we still found worse 
performance at the mid-level difficulties in our control children than we did in adults with normal vision. Performance was further reduced at all difficulty levels in both eyes of strabismics and in the amblyopic eye of anisometropes, consistent with previous reports (Kovacs et al., 2000). Notably, our results for anisometropic children are in close agreement with a prior study of 19 children with anisometropic amblyopia (Chandna et al., 2001). Before Chandna et al., it had been thought that spatial integration in anisometropic amblyopia was unaffected (Hess and Demanins, 1998), but our findings confirm that there is a detectable deficit, at least in children. Coupled with our other psychophysical results, this shows that our amblyopic subjects were comparable to others cited in the literature, and therefore comprise a representative presentation of amblyopia in children of this age range.

\subsubsection{Fixation Stability}

Fixation must be adequately maintained in fMRI studies of vision, particularly for investigating retinotopic organization. Amblyopic patients might be expected to have increased difficulty maintaining fixation, as has been noted previously (Westall and Aslin, 1984). We included eye tracking in our experimental design to address this concern, but unfortunately we were unable to collect eye movement data for a significant proportion of our subjects. However, we did collect other data strongly supporting our claim that the fMRI retinotopic mapping data is not confounded by inadequate fixation.

First, we measured mean across-scan translational head motion in the MRI volumes. In prior work, we have used this measure to show that young children have greater head motion, which can be a confound for retinotopic mapping (Conner et al., 2004). In the current study, we did not find consistent differences in head motion between groups, demonstrating that all subjects cooperated well with the scanning procedure.

Next, we measured responses for a fixation task that was performed throughout all fMRI experiments. Children in both amblyopic groups and controls performed this task with equivalent accuracy, showing that our subjects were paying attention to the stimulus. If subjects had not maintained foveal viewing of the small fixation target, then their response accuracy should have suffered. Therefore, given the available data, we conclude that the subjects in this study were able to lie still in the scanner, and maintained an adequate level of attention to the 
task. Although these data do not prove flawless fixation, they strongly suggest that the children were compliant with the experimental procedure as a whole.

Anecdotally, we actually present indirect evidence that the fixation task accuracy might predict our retinotopic mapping results better than fixation. Despite fixating as well as control subject C1, anisometrope A3 produced a much poorer eccentricity map. However, amblyopic eye acuity, interocular refractive difference, and fixation task accuracy for A3 were by far the worst compared with all other subjects. At least in this case, the poor fixation task accuracy seems to reflect the large acuity deficit and is likely responsible for the poor quality of the eccentricity map.

\subsection{3 fMRI in Children}

Aside from the methodological issues just discussed (i.e., fixation, head motion, and attention), controversy remains whether identical imaging methods used in adults and children yield truly comparable results. The developmental literature using fMRI is rapidly growing, exploring such diverse areas as selective attention (Booth et al., 2004), language representation (Wood et al., 2004), object recognition (Gathers et al., 2004), and reward circuitry (May et al., 2004). In addition, studies are using fMRI to study abnormal developmental processes, such as attention deficit hyperactivity disorder (Booth et al., 2005), dyslexia (Temple et al., 2001), and autism (Brambilla et al., 2004). However, there remain some significant potential confounds for interpreting the developmental literature.

First, adult and pediatric brains are not anatomically or physiologically identical. For example, it is well known children's brains have greater synaptic density and less myelination than adults' brains (Gaillard et al., 2001). Congruent with this idea, initial fMRI studies in infants reported negative activations, opposite of signals found in adults (Yamada et al., 1997; Born et al., 1998). However, these effects were likely specific to the infant brain, as subsequent studies have reported more similar activation patterns in older children and adults (Kang et al., 2003). Furthermore, comparisons of the gross neuroanatomy of the brains of children and adults have not found differences large enough to affect fMRI comparisons (Burgund et al., 2002). Finally, in our previous study of children with normal vision, we found adult-like retinotopic organization in visual cortex (Conner et al., 2004). In summary, some controversy has existed in 
comparing fMRI data from pediatric and adult subjects, but recent studies have strongly suggested that the fMRI BOLD response is functionally mature by the ages examined in the current study.

\subsubsection{Visual Field Remapping}

In a group of adults with amblyopia, we previously demonstrated cortical receptive field remapping at the occipital pole of the brain (I. Conner, J. Odom, T. Schwartz, and J. Mendola, submitted). This significant shift in the visual field representation was found for both strabismic and anisometropic amblyopes, providing a neural characterization of the well-known amblyopic central visual field deficit. This selective psychophysical deficit of central vision has been repeatedly demonstrated in amblyopic adults (Thomas, 1978; Katz et al., 1984), but has thus far remained unreported in children with amblyopia. The current study also did not compare acuity or contrast sensitivity measures in central and peripheral visual fields in children, but these measures would certainly complement the present results. Instead, the current study sought to address whether the shift in visual field representation seen in adults could be found in children with amblyopia.

Eccentricity maps from children's amblyopic eyes did indeed show abnormal organization. In general, maps from strabismic eyes had an overrepresentation of parafoveal and peripheral visual field eccentricities, consistent with the results from the adult study. However, maps from anisometropic eyes were primarily characterized by decreased signal, possibly because acuity was more impaired in the anisometropic children than in the strabismic children. However, it is also important to recognize the range of results. For example, the strabismic eye of subject S1 has a better organized eccentricity map than the right eye (OD) of control subject C1. Thus, these results suggest that the eccentricity mapping function behaves along a continuum, with some overlap between the control and amblyopic groups. Nevertheless, these results are compelling in conjunction with the previous adult study, showing that visual field remapping is measurable even in children whose visual systems are not yet completely mature. 


\subsubsection{Implications for Treating Amblyopia}

Amblyopia treatment is known to be most successful when started early in life, preferably within the first two years. Unfortunately, diagnosis is imperfect in very young children. By the end of the first decade (as in the current study), the prospects for any improvement from patching therapy are lessening, but some improvement is still possible (Mohan et al., 2004; Pediatric Eye Disease Investigator Group, 2004). However, this is the first known investigation of retinotopic organization in children of any age with amblyopia.

The next logical step will be to extend these methods and lessons to younger children. We have established in this and previous studies that the retinotopic organization in visual cortex is adult-like for both normal and amblyopic children in this age range, so it will now become important to determine at what developmental stage the normal and amblyopic brains diverge from one another. Ideally, this work could be conducted in a cohort of children from diagnosis, through treatment, ending in adolescence or adulthood. With this information, fMRI could be used similarly to VEPs to improve diagnosis. Furthermore, the similarity of fMRI results we found in adults and children with amblyopia suggests that data from adults may be relevant to children, who have greater plasticity (I. Conner, J. Odom, T. Schwartz, and J. Mendola, submitted).

In addition, these techniques could be used to help pediatricians and ophthalmologists tailor patching regimens to achieve optimal results, objectively monitoring treatment efficacy while avoiding overpatching, which can in some cases lead to amblyopia of the better eye. Clinicians could also use this retinotopic mapping technique to help evaluate prospective new amblyopia treatments aimed at prolonging or reopening the sensitive period of visual plasticity in older children and adults. Electrophysiological and biochemical studies in animal models of induced amblyopia have helped to refine the concept of the sensitive period and to explore the genetics and molecular mechanisms underlying cortical plasticity (Hensch, 2004). So far, a complex interplay of long-term depression (Heynen et al., 2003), local excitatory-inhibitory circuitry (Fagiolini et al., 2003; Fagiolini et al., 2004), and extracellular matrix regulation (Pizzorusso et al., 2002) has been implicated in the cortical plasticity underlying the sensitive period. Pharmacologic and behavioral therapies aimed at manipulating these processes during the innate window of plasticity, or even reopening the sensitive periods for visual development, are likely in the future. 
For example, behavioral learning methods have shown vision improvement even in adults with amblyopia (Li and Levi, 2004; Polat et al., 2004), although it is not clear at what stage of the visual system this plasticity is taking place. Other investigators have also shown visual improvements with pharmacologic interventions, most prominently levodopa (Chatzistefanou and Mills, 2000; Pandey et al., 2002). Some progress has already been made toward characterizing the effect of levodopa in amblyopic visual cortex, but thus far is inconclusive, alternately finding both enhanced (Yang et al., 2003) and unexpectedly reduced fMRI activation (Rogers, 2003). Further studies are warranted, perhaps building upon the results described in this and our previous study of amblyopic adults. 


\subsection{Conclusions}

We conclude that functional abnormalities of visual cortical areas are a prevalent marker of human amblyopia, even in children. We used fixation monitoring and an attentional task to measure cooperation, methods that should prove useful in further studies of amblyopia. Furthermore, we replicated the results from a previous study in amblyopic adults. In both adults and children with amblyopia, retinotopic mapping of the amblyopic eye showed a shift of parafoveal eccentricity locations toward the occipital pole. This data represents another step toward understanding the neural substrates of amblyopia in human subjects. In the future, these techniques could be used to monitor treatment as vision improves, although it remains uncertain whether the cortical organization would ever really return to "normal" under these circumstances. Future studies should attempt to investigate younger children, for whom successful treatment is more likely because of greater cortical plasticity. 


\subsection{Tables}

Table 1. Subject Characteristics

\begin{tabular}{|c|c|c|c|c|c|c|c|c|c|c|}
\hline $\begin{array}{c}\text { CTL } \\
(\mathrm{N}=3)\end{array}$ & $\begin{array}{c}\text { Age at } \\
\text { Diagnosis } \\
\text { (years) }\end{array}$ & $\begin{array}{c}\text { Patch } \\
\text { Duration } \\
\text { (months) }\end{array}$ & $\begin{array}{c}\text { Age at } \\
\text { Surgery } \\
\text { (years) }\end{array}$ & $\begin{array}{l}\text { Current } \\
\text { Deviation } \\
\text { (prism } \\
\text { diopters) }\end{array}$ & & $\begin{array}{c}\text { OS } \\
\text { VA } \\
\text { (MAR) }\end{array}$ & $\begin{array}{l}\text { Near } \\
\text { VA Diff } \\
\text { (MAR) }\end{array}$ & $\begin{array}{c}\text { Far } \\
\text { VA Diff } \\
\text { (MAR) }\end{array}$ & $\begin{array}{c}\text { IRD } \\
\text { (diopters) }\end{array}$ & $\begin{array}{l}\text { Stereo- } \\
\text { acuity } \\
\text { (arc-sec) }\end{array}$ \\
\hline $\mathbf{C}$ C1 & - & - & - & - & - & 1.00 & 0.00 & 0.00 & 1.25 & 40 \\
\hline$\bullet \mathrm{C} 2$ & - & - & - & - & - & 1.00 & 0.00 & 0.00 & 0.25 & 40 \\
\hline$\Delta \mathrm{C} 3$ & - & - & - & - & - & 1.00 & 0.00 & 0.00 & 0.25 & 40 \\
\hline Mean & - & - & - & - & - & 1.00 & 0.00 & 0.00 & 0.58 & - \\
\hline & & & & & & & & & & \\
\hline $\begin{array}{c}\text { STRAB } \\
(\mathrm{N}=3)\end{array}$ & $\begin{array}{c}\text { Age at } \\
\text { Diagnosis } \\
\text { (years) }\end{array}$ & $\begin{array}{l}\text { Patch } \\
\text { Duration } \\
\text { (months) }\end{array}$ & $\begin{array}{l}\text { Age at } \\
\text { Surgery } \\
\text { (years) }\end{array}$ & $\begin{array}{l}\text { Current } \\
\text { Deviation } \\
\text { (prism } \\
\text { diopters) }\end{array}$ & $A E$ & $\begin{array}{c}\mathrm{AE} \\
\mathrm{VA} \\
\text { (MAR) }\end{array}$ & $\begin{array}{l}\text { Near } \\
\text { VA Diff } \\
\text { (MAR) }\end{array}$ & $\begin{array}{c}\text { Far } \\
\text { VA Diff } \\
\text { (MAR) }\end{array}$ & $\begin{array}{c}\text { IRD } \\
\text { (diopters) }\end{array}$ & $\begin{array}{c}\text { Stereo- } \\
\text { acuity } \\
\text { (arc-sec) }\end{array}$ \\
\hline 口 S1 & 5 & 0 & - & 20 & OS & 2.00 & 0.75 & 0.00 & 2.00 & $>3500$ \\
\hline - S2 & 6 & 14 & - & 0 & OD & 1.00 & 0.00 & 0.75 & 0.50 & 3500 \\
\hline$\Delta$ S3 & 5 & 6 & - & 3 & OD & 3.15 & 0.65 & 0.65 & 0.25 & 3500 \\
\hline Mean & 5.3 & 6.7 & - & 7.7 & - & 2.05 & 0.47 & 0.47 & 0.92 & - \\
\hline & & & & & & & & & & \\
\hline $\begin{array}{c}\text { ANISO } \\
(\mathrm{N}=3)\end{array}$ & $\begin{array}{l}\text { Age at } \\
\text { Diagnosis } \\
\text { (years) }\end{array}$ & $\begin{array}{c}\text { Patch } \\
\text { Duration } \\
\text { (months) }\end{array}$ & $\begin{array}{l}\text { Age at } \\
\text { Surgery } \\
\text { (years) }\end{array}$ & $\begin{array}{l}\text { Current } \\
\text { Deviation } \\
\text { (prism } \\
\text { diopters) }\end{array}$ & $A E$ & $\begin{array}{c}\mathrm{AE} \\
\mathrm{VA} \\
\text { (MAR) }\end{array}$ & $\begin{array}{l}\text { Near } \\
\text { VA Diff } \\
\text { (MAR) }\end{array}$ & $\begin{array}{c}\text { Far } \\
\text { VA Diff } \\
\text { (MAR) }\end{array}$ & $\begin{array}{c}\text { IRD } \\
\text { (diopters) }\end{array}$ & $\begin{array}{c}\text { Stereo- } \\
\text { acuity } \\
\text { (arc-sec) }\end{array}$ \\
\hline a1 & 8 & 12 & - & - & OS & 2.50 & 1.70 & 1.70 & 1.50 & 60 \\
\hline - $\mathrm{A} 2$ & 4 & 48 & - & - & OD & 5.00 & 4.00 & 3.00 & 2.50 & $>3500$ \\
\hline$\Delta \mathrm{A} 3$ & 9 & 1.5 & - & - & os & 12.50 & 10.90 & 9.00 & 3.50 & $>3500$ \\
\hline Mean & 7.0 & 20.5 & - & - & - & 6.67 & 5.53 & 4.57 & 2.50 & - \\
\hline
\end{tabular}

VA - visual acuity, reported as minimum angle of resolution (MAR) in arc-minutes.

IRD - interocular refractive difference, diopters of spherical equivalent.

$\mathrm{AE}$ - amblyopic eye. 


\subsection{Figures}

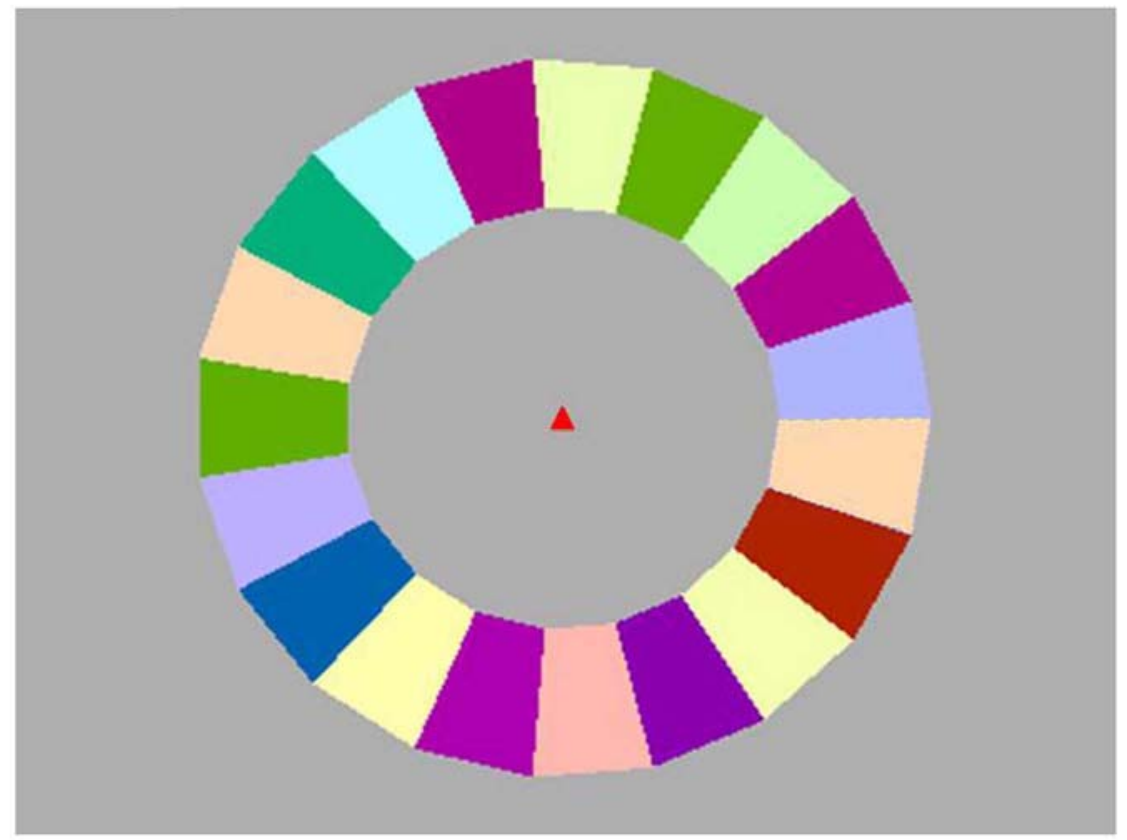

FIGURE 5-1. FMRI STIMULI. The subject maintained fixation on a central "arrowhead" target. Attention was monitored by recording a button-press each time the arrowhead changed orientation. The eccentricity stimulus was presented separately to each eye in paired expansion/contraction scans. See Methods for further details. 
A.
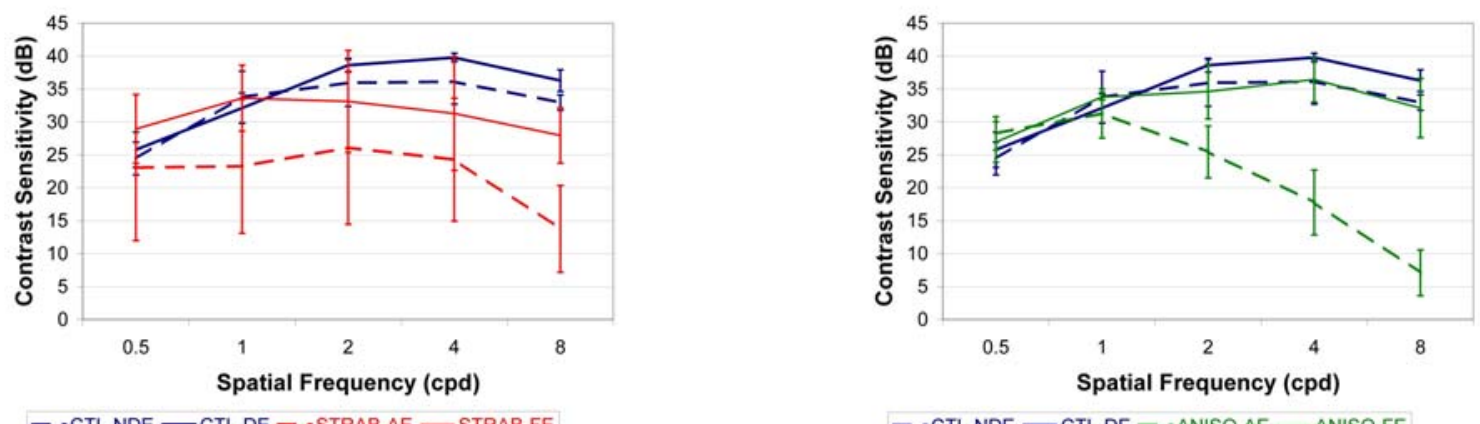

B.
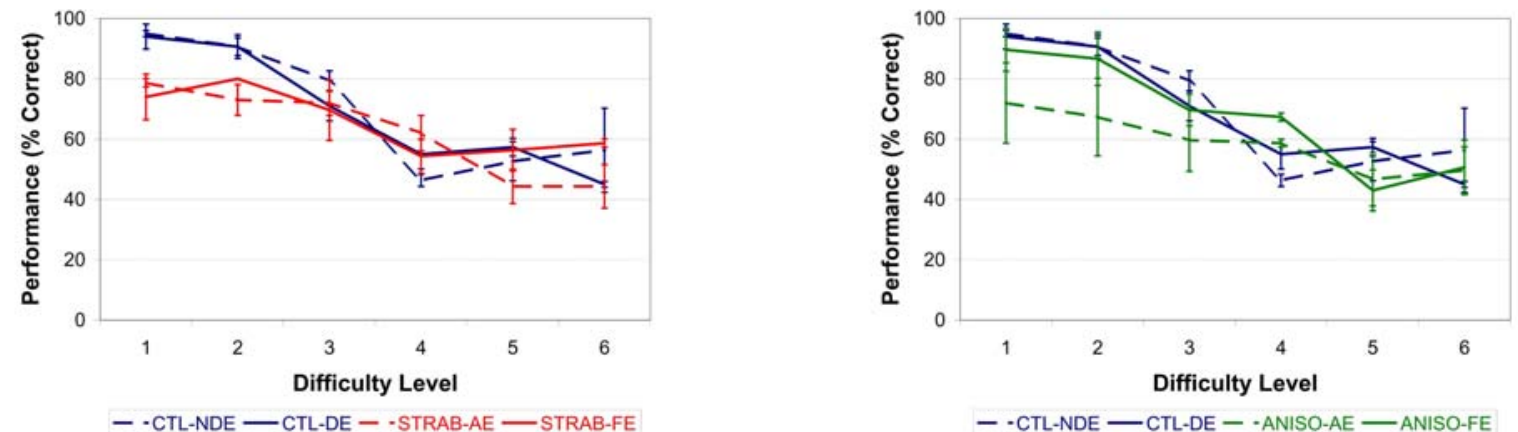

Figure 5-2. Psychophysical Testing. A. Contrast sensitivity was depressed in strabismic and anisometropic amblyopes at high spatial frequencies. B. Contour integration was impaired for amblyopic and fellow eyes in strabismics, but for amblyopic eyes only in anisometropes. 
A.

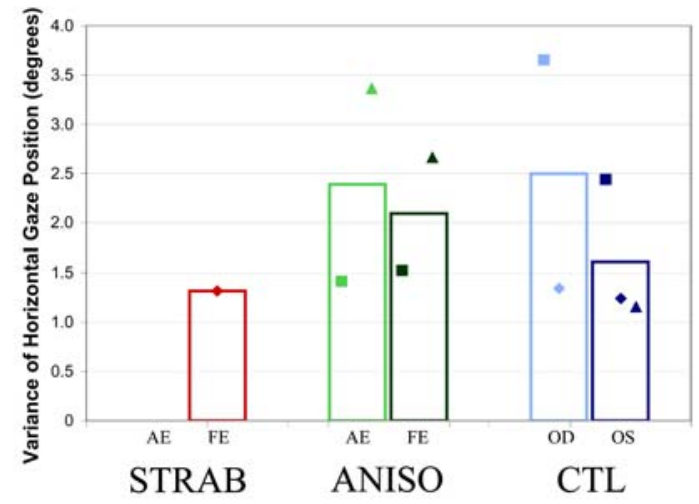

B.

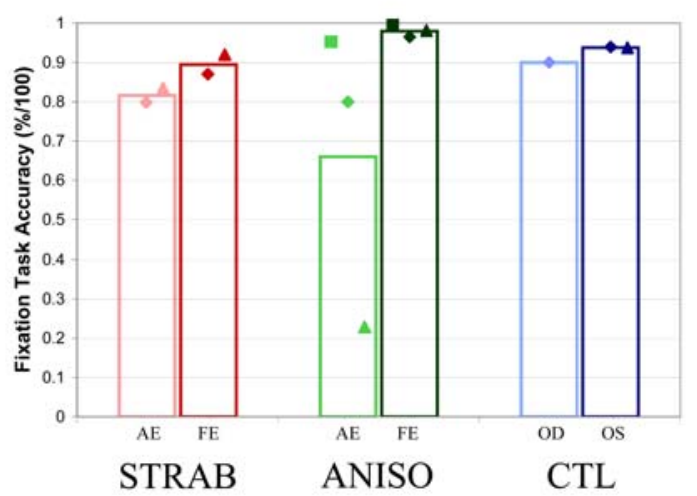

Figure 5-3. Fixation Stability and Response Accuracy. A. Fixation stability was similar across groups for the monitored subjects. B. Fixation task response accuracy was high for most subjects, regardless of eye used. Group mean (open bars) and individual data are shown, with saturated colors corresponding to the fellow eye (FE) and lighter shades to the amblyopic eye (AE). The right eye (OD) in control subjects is used for comparison with amblyopic eyes. 


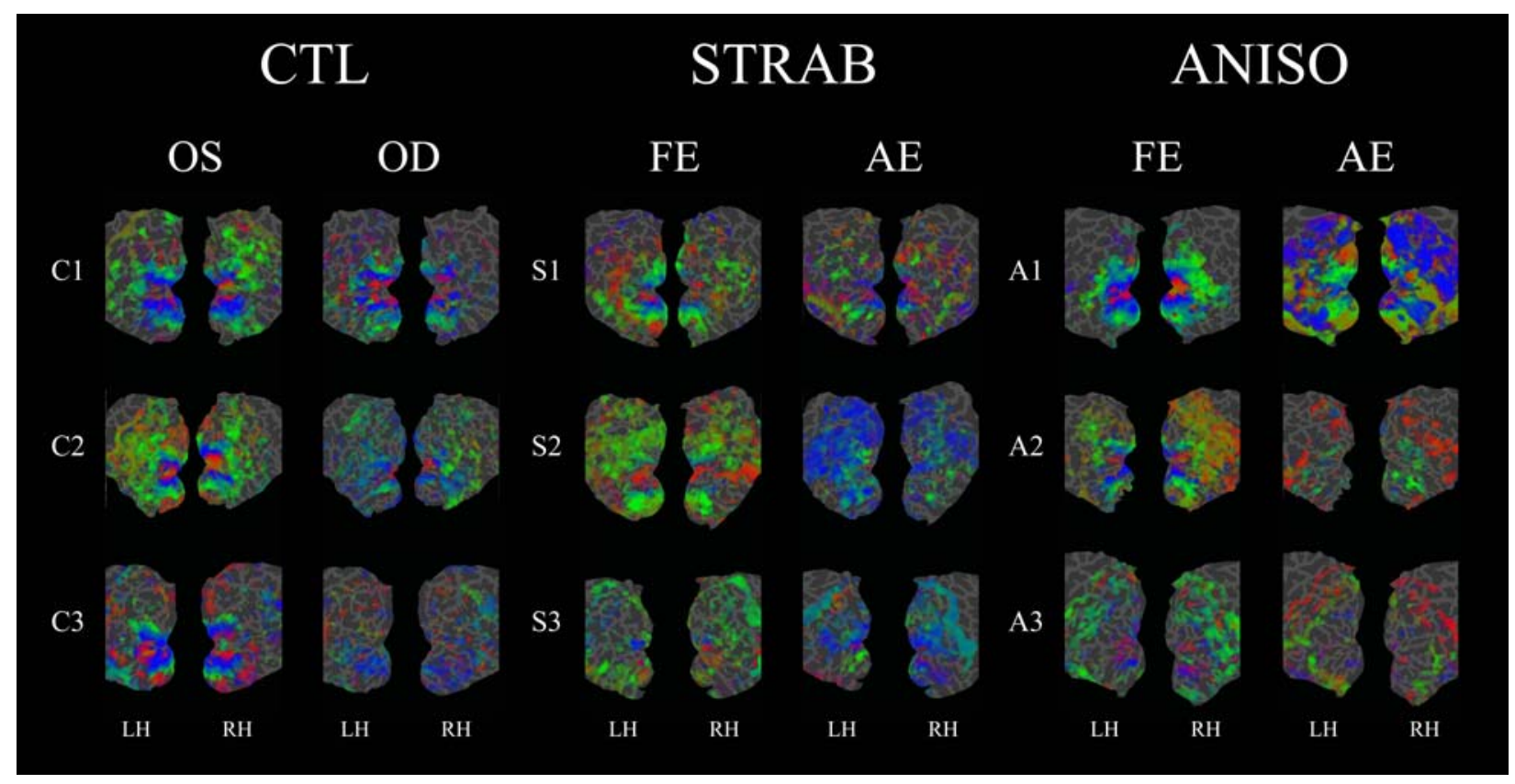

Figure 5-4. ECCENTRICITY MAPS FROM SiX AMBLYOPIC AND THREE CONTROL CHILDREN.

Eccentricity maps are shown on left and right hemisphere flattened occipital poles for fellow and amblyopic eyes of the six patients and three controls. These maps are arranged in order from most (top) to least (bottom) normal in appearance. Red, blue, and green indicate the central 0 $2.5 \mathrm{deg}, 2.5-8.5 \mathrm{deg}$, and 8.5-15 deg eccentricity, respectively. Note the trend for more organized arrangement of colors in the fellow eyes vs. the disorganized patterns in the amblyopic eyes. Light and dark gray regions indicate the unfolded gyri and sulci, respectively. 


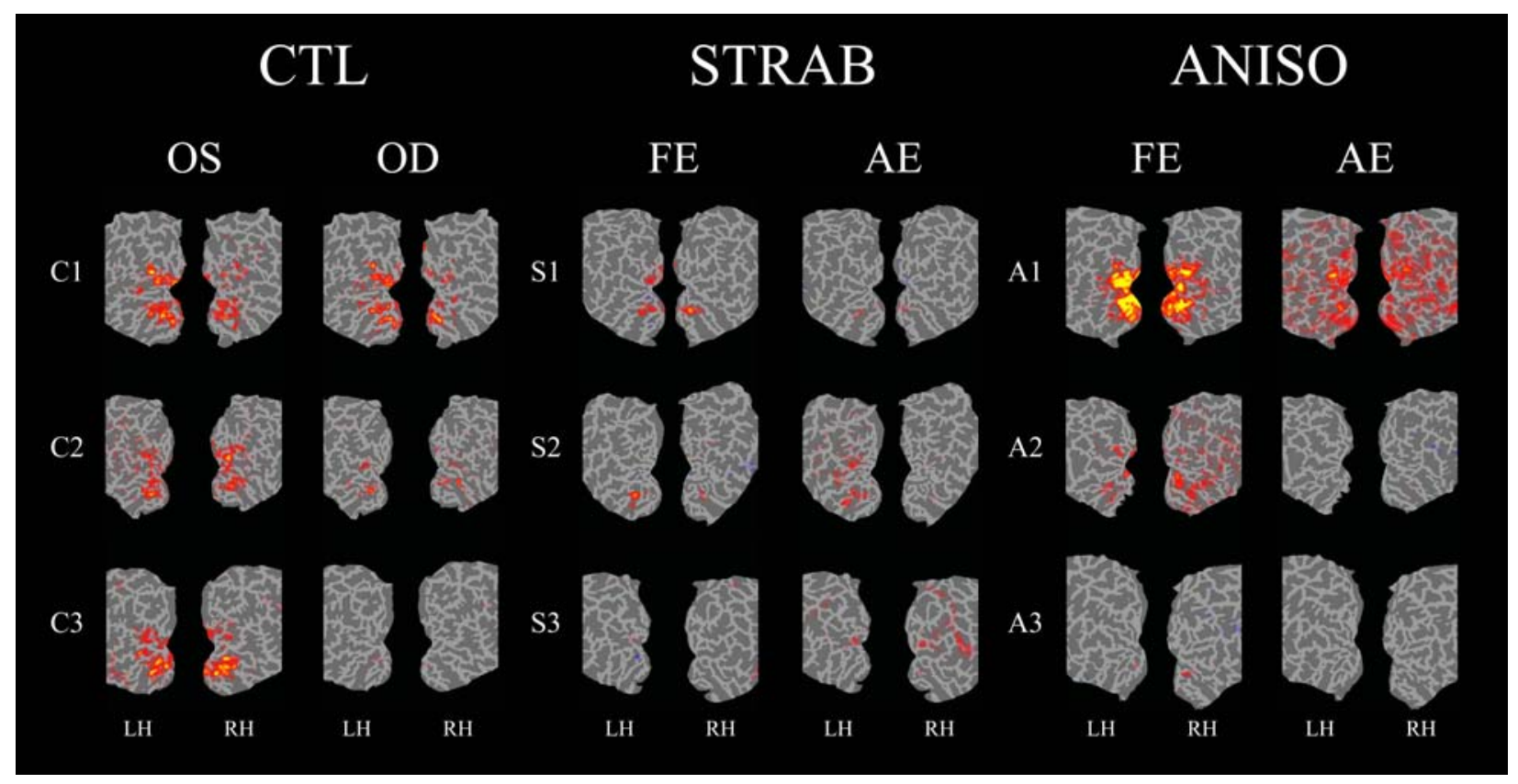

Figure 5-5. SignifiCANCE MAPS From SiX AMBlyopiC AND ThreE CONTROL Children. The significance of the Fourier signal is plotted for comparison across subjects $(0.0001<p<0.05)$. Most amblyopic brains have less signal compared with controls, as well as for amblyopic eye compared with fellow eye stimulation. 


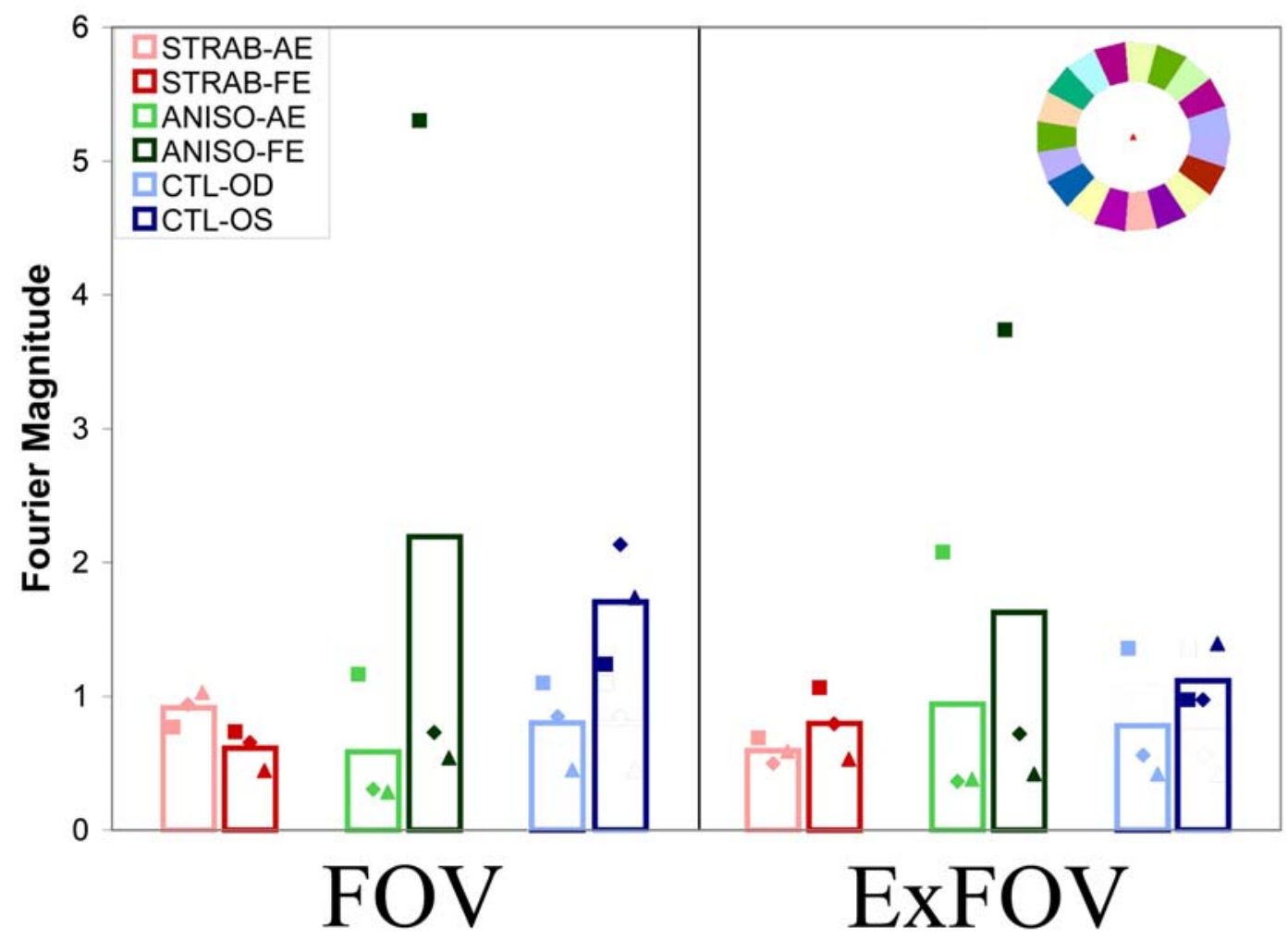

Figure 5-6. Fourier Signal Magnitude is Depressed for Amblyopic Eye Viewing. Mean Fourier magnitude is depressed for amblyopic compared with control and fellow eyes, except for FOV in strabismics, which have equivalent interocular signal. 


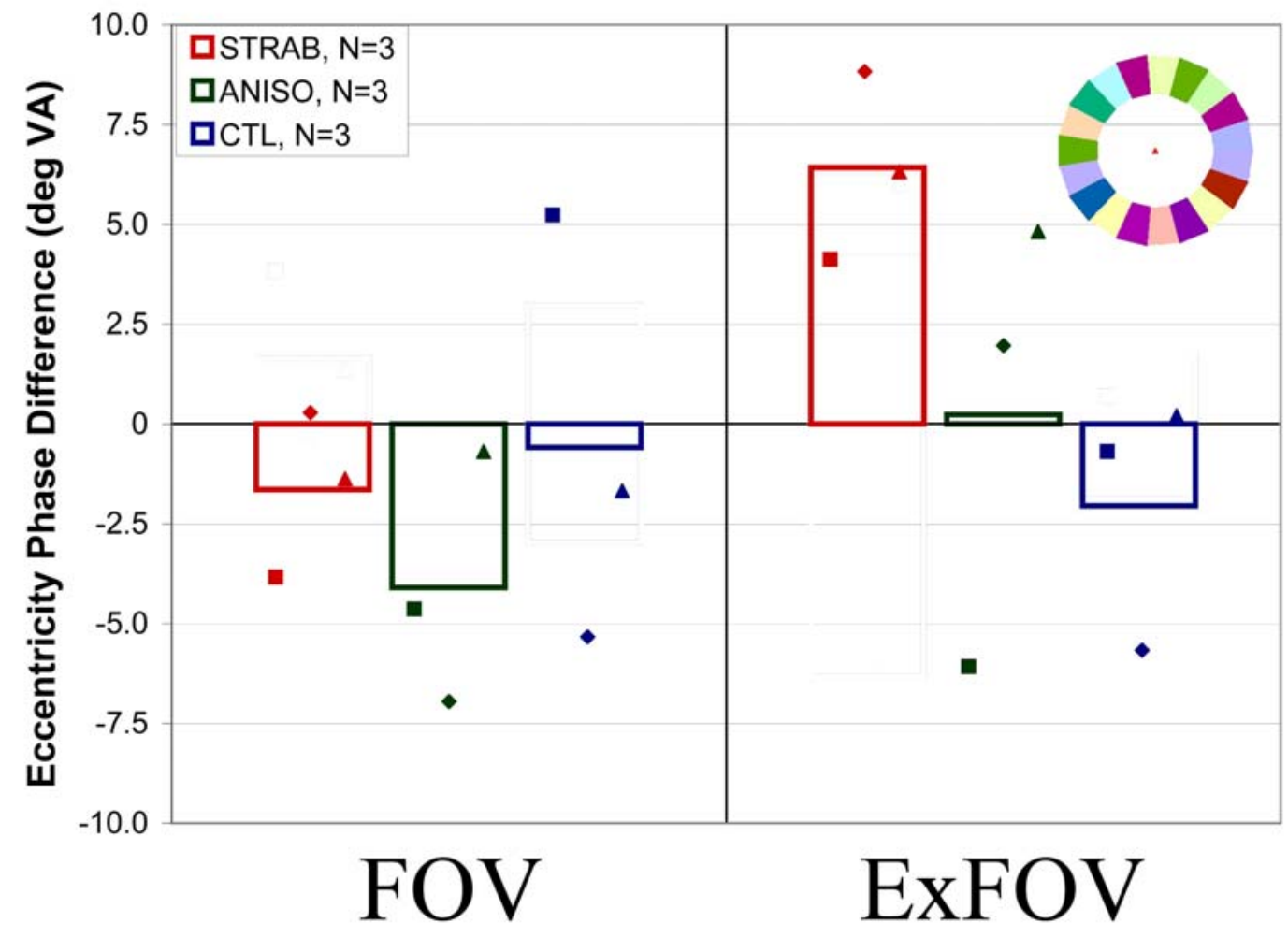

Figure 5-7. ECCENTRICITY PHASE IS SHIfTED FOR AMBlyopic SubJeCtS. Mean interocular phase difference was negative for both amblyopic groups in FOV, but positive for strabismics in ExFOV. 


\subsection{References}

Abrahamsson M, Sjostrand J (1988) Contrast sensitivity and acuity relationship in strabismic and anisometropic amblyopia. Br J Ophthalmol 72:44-49.

Adams RJ, Courage ML (2002) Using a single test to measure human contrast sensitivity from early childhood to maturity. Vision Res 42:1205-1210.

Algaze A, Roberts C, Leguire L, Schmalbrock P, Rogers G (2002) Functional magnetic resonance imaging as a tool for investigating amblyopia in the human visual cortex: a pilot study. J AAPOS 6:300-308.

Asper L, Crewther D, Crewther SG (2000) Strabismic amblyopia. Part 1. Psychophysics. Clin Exp Optom 83:49-58.

Atkinson J, Braddick O, Pimm-Smith E (1982) 'Preferential looking' for monocular and binocular acuity testing of infants. Br J Ophthalmol 66:264-268.

Barnes GR, Hess RF, Dumoulin SO, Achtman RL, Pike GB (2001) The cortical deficit in humans with strabismic amblyopia. J Physiol 533:281-297.

Birch EE, Gwiazda J, Held R (1982) Stereoacuity development for crossed and uncrossed disparities in human infants. Vision Res 22:507-513.

Birch EE, Stager DR (1985) Monocular acuity and stereopsis in infantile esotropia. Invest Ophthalmol Vis Sci 26:1624-1630.

Birch EE, Stager DR (1996) The critical period for surgical treatment of dense congenital unilateral cataract. Invest Ophthalmol Vis Sci 37:1532-1538.

Birch EE, Swanson WH (2000) Hyperacuity deficits in anisometropic and strabismic amblyopes with known ages of onset. Vision Res 40:1035-1040.

Bodis-Wollner I (1980) Detection of visual defects using the contrast sensitivity function. Int Ophthalmol Clin 20:135-153.

Booth JR, Burman DD, Meyer JR, Lei Z, Trommer BL, Davenport ND, Li W, Parrish TB, Gitelman DR, Mesulam MM (2005) Larger deficits in brain networks for response inhibition than for visual selective attention in attention deficit hyperactivity disorder (ADHD). J Child Psychol Psychiatry 46:94-111.

Booth JR, Burman DD, Meyer JR, Trommer BL, Davenport ND, Parrish TB, Gitelman DR, Mesulam MM (2004) Brain-behavior correlation in children depends on the neurocognitive network. Hum Brain Mapp 23:99-108. 
Born P, Leth H, Miranda MJ, Rostrup E, Stensgaard A, Peitersen B, Larsson HB, Lou HC (1998) Visual activation in infants and young children studied by functional magnetic resonance imaging. Pediatr Res 44:578-583.

Bradley A, Freeman RD (1982) Contrast sensitivity in children. Vision Res 22:953-959.

Bradley A, Freeman RD (1981) Contrast sensitivity in anisometropic amblyopia. Invest Ophthalmol Vis Sci 21:467-476.

Brainard DH (1997) The Psychophysics Toolbox. Spat Vis 10:433-436.

Brambilla P, Hardan AY, di Nemi SU, Caverzasi E, Soares JC, Perez J, Barale F (2004) The functional neuroanatomy of autism. Funct Neurol 19:9-17.

Braun J (1999) On the detection of salient contours. Spat Vis 12:211-225.

Burgund ED, Kang HC, Kelly JE, Buckner RL, Snyder AZ, Petersen SE, Schlaggar BL (2002) The feasibility of a common stereotactic space for children and adults in fMRI studies of development. Neuroimage 17:184-200.

Campos EC, Prampolini ML, Gulli R (1984) Contrast sensitivity differences between strabismic and anisometropic amblyopia: objective correlate by means of visual evoked responses. Doc Ophthalmol 58:45-50.

Chandna A, Pennefather PM, Kovacs I, Norcia AM (2001) Contour integration deficits in anisometropic amblyopia. Invest Ophthalmol Vis Sci 42:875-878.

Chapman B, Godecke I, Bonhoeffer T (1999) Development of orientation preference in the mammalian visual cortex. J Neurobiol 41:18-24.

Chatzistefanou KI, Mills MD (2000) The role of drug treatment in children with strabismus and amblyopia. Paediatr Drugs 2:91-100.

Ciuffreda KJ, Fisher SK (1987) Impairment of contrast discrimination in amblyopic eyes. Ophthalmic Physiol Opt 7:461-467.

Conner IP, Sharma S, Lemieux SK, Mendola JD (2004) Retinotopic organization in children measured with fMRI. J Vis 4:509-523.

Cox RW, Jesmanowicz A (1999) Real-time 3D image registration for functional MRI. Magn Reson Med 42:1014-1018.

Dale AM, Fischl B, Sereno MI (1999) Cortical surface-based analysis. I. Segmentation and surface reconstruction. Neuroimage 9:179-194.

Dale AM, Sereno MI (1993) Improved localization of cortical activity by combining EEG and MEG with MRI cortical surface reconstruction. J Cogn Neurosci 5:162-176. 
Daw NW (1994) Mechanisms of plasticity in the visual cortex. The Friedenwald Lecture. Invest Ophthalmol Vis Sci 35:4168-4179.

Demer JL, Grafton S, Marg E, Mazziotta JC, Nuwer M (1997) Positron-emission tomographic study of human amblyopia with use of defined visual stimuli. J AAPOS 1:158-171.

Ellemberg D, Lewis TL, Liu CH, Maurer D (1999) Development of spatial and temporal vision during childhood. Vision Res 39:2325-2333.

Ellemberg D, Lewis TL, Maurer D, Brent HP (2000) Influence of monocular deprivation during infancy on the later development of spatial and temporal vision. Vision Res 40:3283-3295.

Engel SA, Glover GH, Wandell BA (1997) Retinotopic organization in human visual cortex and the spatial precision of functional MRI. Cereb Cortex 7:181-192.

Fagiolini M, Fritschy JM, Low K, Mohler H, Rudolph U, Hensch TK (2004) Specific GABAA circuits for visual cortical plasticity. Science 303:1681-1683.

Fagiolini M, Katagiri H, Miyamoto H, Mori H, Grant SG, Mishina M, Hensch TK (2003) Separable features of visual cortical plasticity revealed by N-methyl-D-aspartate receptor $2 \mathrm{~A}$ signaling. Proc Natl Acad Sci U S A 100:2854-2859.

Field DJ, Hayes A, Hess RF (1993) Contour integration by the human visual system: evidence for a local "association field". Vision Res 33:173-193.

Fischl B, Liu A, Dale AM (2001) Automated manifold surgery: constructing geometrically accurate and topologically correct models of the human cerebral cortex. IEEE Trans Med Imaging 20:70-80.

Fischl B, Sereno MI, Dale AM (1999) Cortical surface-based analysis. II: Inflation, flattening, and a surface-based coordinate system. Neuroimage 9:195-207.

Fronius M, Sireteanu R, Zubcov A (2004) Deficits of spatial localization in children with strabismic amblyopia. Graefes Arch Clin Exp Ophthalmol.

Gaillard WD, Grandin CB, Xu B (2001) Developmental aspects of pediatric fMRI: considerations for image acquisition, analysis, and interpretation. Neuroimage 13:239-249.

Gathers AD, Bhatt R, Corbly CR, Farley AB, Joseph JE (2004) Developmental shifts in cortical loci for face and object recognition. Neuroreport 15:1549-1553.

Glover G (1999) Simple analytic spiral K-space algorithm. Magn Reson Med 42:412-415.

Goodyear BG, Nicolle DA, Humphrey GK, Menon RS (2000) BOLD fMRI response of early visual areas to perceived contrast in human amblyopia. J Neurophysiol 84:1907-1913. 
Harwerth RS, Smith EL, III, Boltz RL, Crawford ML, von Noorden GK (1983) Behavioral studies on the effect of abnormal early visual experience in monkeys: spatial modulation sensitivity. Vision Res 23:1501-1510.

Harwerth RS, Smith EL, III, Duncan GC, Crawford ML, von Noorden GK (1986) Multiple sensitive periods in the development of the primate visual system. Science 232:235-238.

Henc-Petrinovic L, Deban N, Gabric N, Petrinovic J (1993) Prognostic value of visual evoked responses in childhood amblyopia. Eur J Ophthalmol 3:114-120.

Hensch TK (2004) Critical period regulation. Annu Rev Neurosci 27:549-579.

Hess RF, Demanins R (1998) Contour integration in anisometropic amblyopia. Vision Res 38:889-894.

Hess RF, Howell ER (1977) The threshold contrast sensitivity function in strabismic amblyopia: evidence for a two type classification. Vision Res 17:1049-1055.

Heynen AJ, Yoon BJ, Liu CH, Chung HJ, Huganir RL, Bear MF (2003) Molecular mechanism for loss of visual cortical responsiveness following brief monocular deprivation. Nat Neurosci 6:854-862.

Howell ER, Mitchell DE, Keith CG (1983) Contrast thresholds for sine gratings of children with amblyopia. Invest Ophthalmol Vis Sci 24:782-787.

Imamura K, Richter H, Fischer H, Lennerstrand G, Franzen O, Rydberg A, Andersson J, Schneider H, Onoe H, Watanabe Y, Langstrom B (1997) Reduced activity in the extrastriate visual cortex of individuals with strabismic amblyopia. Neurosci Lett 225:173-176.

Ing MR, Pang SW (1974) The racial distribution of strabismus. A statistical study. Hawaii Med J $33: 22-23$.

Kang HC, Burgund ED, Lugar HM, Petersen SE, Schlaggar BL (2003) Comparison of functional activation foci in children and adults using a common stereotactic space. Neuroimage 19:1628.

Katz LM, Levi DM, Bedell HE (1984) Central and peripheral contrast sensitivity in amblyopia with varying field size. Doc Ophthalmol 58:351-373.

Kiorpes L, Boothe RG, Hendrickson AE, Movshon JA, Eggers HM, Gizzi MS (1987) Effects of early unilateral blur on the macaque's visual system. I. Behavioral observations. J Neurosci 7:1318-1326.

Kiorpes L, Kiper DC, O'Keefe LP, Cavanaugh JR, Movshon JA (1998) Neuronal correlates of amblyopia in the visual cortex of macaque monkeys with experimental strabismus and anisometropia. J Neurosci 18:6411-6424. 
Kovacs I, Julesz B (1993) A closed curve is much more than an incomplete one: effect of closure in figure-ground segmentation. Proc Natl Acad Sci U S A 90:7495-7497.

Kovacs I, Kozma P, Feher A, Benedek G (1999) Late maturation of visual spatial integration in humans. Proc Natl Acad Sci U S A 96:12204-12209.

Kovacs I, Polat U, Pennefather PM, Chandna A, Norcia AM (2000) A new test of contour integration deficits in patients with a history of disrupted binocular experience during visual development. Vision Res 40:1775-1783.

Kwong KK, Belliveau JW, Chester DA, Goldberg IE, Weiskoff RM, Poncelet BP, Kennedy DN, Hoppel BE, Cohen MS, Turner R, Rosen B, Brady TJ (1992) Dynamic magnetic resonce imaging of human brain activity during primary sensory stimulation. Proc Natl Acad Sci U S A 89:5675-5679.

Lee KM, Lee SH, Kim NY, Kim CY, Sohn JW, Choi MY, Gyu CD, Hwang JM, Ho PK, Lee DS, Suk YY, Hyun CK (2001) Binocularity and spatial frequency dependence of calcarine activation in two types of amblyopia. Neurosci Res 40:147-153.

Levi DM, Harwerth RS (1978) Contrast evoked potentials in strabismic and anisometropic amblyopia. Invest Ophthalmol Vis Sci 17:571-575.

Levi DM, Klein S (1982) Hyperacuity and amblyopia. Nature 298:268-270.

Levi DM, Klein SA (1985) Vernier acuity, crowding and amblyopia. Vision Res 25:979-991.

Levi DM, Klein SA, Sharma V (1999) Position jitter and undersampling in pattern perception. Vision Res 39:445-465.

Lewis TL, Maurer D (2005) Multiple sensitive periods in human visual development: Evidence from visually deprived children. Dev Psychobiol 46:163-183.

Li RW, Levi DM (2004) Characterizing the mechanisms of improvement for position discrimination in adult amblyopia. J Vis 4:476-487.

Liu GT, Miki A, Francis E, Quinn GE, Modestino EJ, Bonhomme GR, Haselgrove JC (2004) Eye dominance in visual cortex in amblyopia using functional magnetic resonance imaging. $\mathrm{J}$ AAPOS 8:184-186.

Lundh BL, Lennerstrand G (1983) Effects of amblyopia therapy on contrast sensitivity as reflected in the visuogram. Acta Ophthalmol (Copenh) 61:431-446.

Magnusson G, Abrahamsson M, Sjostrand J (2002) Changes in visual acuity from 4 to 12 years of age in children operated for bilateral congenital cataracts. Br J Ophthalmol 86:1385-1389.

Maurer D, Lewis TL (2001) Visual acuity: the role of visual input in inducing postnatal change. Clin Neurosci Res 1:239-247. 
Maurer D, Lewis TL, Brent HP, Levin AV (1999) Rapid improvement in the acuity of infants after visual input. Science 286:108-110.

May JC, Delgado MR, Dahl RE, Stenger VA, Ryan ND, Fiez JA, Carter CS (2004) Event-related functional magnetic resonance imaging of reward-related brain circuitry in children and adolescents. Biol Psychiatry 55:359-366.

Mayer DL, Dobson V (1982) Visual acuity development in infants and young children, as assessed by operant preferential looking. Vision Res 22:1141-1151.

McKee SP, Levi DM, Movshon JA (2003) The pattern of visual deficits in amblyopia. J Vis 3:380-405.

Mendola JD, Conner IP, Roy A, Chan ST, Schwartz TL, Odom JV, Kwong KK (2005) Voxelbased analysis of MRI detects abnormal visual cortex in children and adults with amblyopia. Hum Brain Mapp 25:222-236.

Mendola JD, Dale AM, Fischl B, Liu AK, Tootell RB (1999) The representation of illusory and real contours in human cortical visual areas revealed by functional magnetic resonance imaging. J Neurosci 19:8560-8572.

Mitchell DE, MacKinnon S (2002) The present and potential impact of research on animal models for clinical treatment of stimulus deprivation amblyopia. Clin Exp Optom 85:5-18.

Mizoguchi S, Suzuki Y, Kiyosawa M, Mochizuki M, Ishii K (2005) Differential activation of cerebral blood flow by stimulating amblyopic and fellow eye. Graefes Arch Clin Exp Ophthalmol.

Mohan K, Saroha V, Sharma A (2004) Successful occlusion therapy for amblyopia in 11- to 15year-old children. J Pediatr Ophthalmol Strabismus 41:89-95.

Norcia AM, Tyler CW, Hamer RD (1990) Development of contrast sensitivity in the human infant. Vision Res 30:1475-1486.

Ogawa S, Tank W, Menon RS, Ellermann JM, Kim SG, Merkle H, Ugurbil K (1992) Intrinsic signal changes accompanying sensory stimulation: functional brain mapping with magnetic resonce imaging. Proc Natl Acad Sci U S A 89:5951-5955.

Pandey PK, Chaudhuri Z, Kumar M, Satyabala K, Sharma P (2002) Effect of levodopa and carbidopa in human amblyopia. J Pediatr Ophthalmol Strabismus 39:81-89.

Pediatric Eye Disease Investigator Group (2004) A prospective, pilot study of treatment of amblyopia in children 10 to $<18$ years old. Am J Ophthalmol 137:581-583.

Pelli DG (1997) The VideoToolbox software for visual psychophysics: transforming numbers into movies. Spat Vis 10:437-442. 
Pennefather PM, Chandna A, Kovacs I, Polat U, Norcia AM (1999) Contour detection threshold: repeatability and learning with 'contour cards'. Spat Vis 12:257-266.

Pizzorusso T, Medini P, Berardi N, Chierzi S, Fawcett JW, Maffei L (2002) Reactivation of ocular dominance plasticity in the adult visual cortex. Science 298:1248-1251.

Polat U, Ma-Naim T, Belkin M, Sagi D (2004) Improving vision in adult amblyopia by perceptual learning. Proc Natl Acad Sci U S A 101:6692-6697.

Rogers GL (2003) Functional magnetic resonance imaging (fMRI) and effects of L-dopa on visual function in normal and amblyopic subjects. Trans Am Ophthalmol Soc 101:401-415.

Rogers GL, Bremer DL, Leguire LE (1987) The contrast sensitivity function and childhood amblyopia. Am J Ophthalmol 104:64-68.

Schor CM (1985) Development of stereopsis depends upon contrast sensitivity and spatial tuning. J Am Optom Assoc 56:628-635.

Sereno MI, Dale AM, Reppas JB, Kwong KK, Belliveau JW, Brady TJ, Rosen BR, Tootell RB (1995) Borders of multiple visual areas in humans revealed by functional magnetic resonance imaging. Science 268:889-893.

Sereno MI, McDonald CT, Allman JM (1994) Analysis of retinotopic maps in extrastriate cortex. Cereb Cortex 4:601-620.

Sharma V, Levi DM, Klein SA (2000) Undercounting features and missing features: evidence for a high-level deficit in strabismic amblyopia. Nat Neurosci 3:496-501.

Sireteanu R, Fronius M (1990) Human amblyopia: structure of the visual field. Exp Brain Res 79:603-614.

Skoczenski AM, Norcia AM (2002) Late maturation of visual hyperacuity. Psychol Sci 13:537541.

Skoczenski AM, Norcia AM (1999) Development of VEP Vernier acuity and grating acuity in human infants. Invest Ophthalmol Vis Sci 40:2411-2417.

Slater A, Morison V, Somers M (1988) Orientation discrimination and cortical function in the human newborn. Perception 17:597-602.

Smith EL, III, Harwerth RS, Crawford ML (1985) Spatial contrast sensitivity deficits in monkeys produced by optically induced anisometropia. Invest Ophthalmol Vis Sci 26:330-342.

Sokol S (1983) Abnormal evoked potential latencies in amblyopia. Br J Ophthalmol 67:310-314.

Temple E, Poldrack RA, Salidis J, Deutsch GK, Tallal P, Merzenich MM, Gabrieli JD (2001) Disrupted neural responses to phonological and orthographic processing in dyslexic children: an fMRI study. Neuroreport 12:299-307. 
Thomas J (1978) Normal and amblyopic contrast sensitivity function in central and peripheral retinas. Invest Ophthalmol Vis Sci 17:746-753.

Thorn F, Gwiazda J, Cruz AA, Bauer JA, Held R (1994) The development of eye alignment, convergence, and sensory binocularity in young infants. Invest Ophthalmol Vis Sci 35:544553.

Vaegan, Taylor D (1979) Critical period for deprivation amblyopia in children. Trans Ophthalmol Soc U K 99:432-439.

Westall CA, Aslin RN (1984) Fixational eye movements and autokinesis in amblyopes. Ophthalmic Physiol Opt 4:333-337.

White LE, Coppola DM, Fitzpatrick D (2001) The contribution of sensory experience to the maturation of orientation selectivity in ferret visual cortex. Nature 411:1049-1052.

Wiesel TN, Hubel DH (1963) Single-cell responses in striate cortex of kittens deprived of vision in one eye. J Neurophysiol 26:1003-1017.

Wood AG, Harvey AS, Wellard RM, Abbott DF, Anderson V, Kean M, Saling MM, Jackson GD (2004) Language cortex activation in normal children. Neurology 63:1035-1044.

Yamada H, Sadato N, Konishi Y, Kimura K, Tanaka M, Yonekura Y, Ishii Y (1997) A rapid brain metabolic change in infants detected by fMRI. Neuroreport 8:3775-3778.

Yang CI, Yang ML, Huang JC, Wan YL, Jui-Fang TR, Wai YY, Liu HL (2003) Functional MRI of amblyopia before and after levodopa. Neurosci Lett 339:49-52. 
CHAPTER 6: General Discussion 


\subsection{Summary and Significance of the Current Project}

Amblyopia is a common developmental disorder of vision, affecting from 1-3\% of the population (Flom and Neumaier, 1966; Hillis et al., 1983; Ohlsson et al., 2001; Ohlsson et al., 2003). It is associated with an obstruction to normal vision early in postnatal development, and can lead to irreversibly impaired vision in one eye if not detected and treated early in life (von Noorden and Campos, 2001). Binocular vision, characterized by stereopsis and depth perception, is universally absent in amblyopia. This has the potential to influence career and recreational choices, and these lost opportunities can have a significant social and economic impact throughout a person's life (Chua and Mitchell, 2004). In addition, the incidence of functional blindness due to loss of the good eye is at least three times greater for patients with amblyopia (Tommila and Tarkkanen, 1981). For these practical and clinical reasons, amblyopia is an important and relevant disorder for study.

More broadly, amblyopia serves as a useful example of neural plasticity in humans. As first noted by Hubel and Wiesel in kittens, eyelid suture during early development resulted in dramatic anatomical and physiological abnormalities in visual cortex (Wiesel and Hubel, 1963). This form of monocular deprivation (an analog of the condition that occurs in humans with a congenital cataract) resulted in shifted ocular dominance in primary visual cortex, the first site in the visual system where input from the two eyes is combined (Hubel et al., 1977). Analogs of other forms of human amblyopia have also found similar results (Hubel and Wiesel, 1965). Evidence that these changes were rooted in competition of monocular inputs at the cortical synaptic level was provided with the finding that early binocular deprivation did not lead to

ocular dominance shifts, and in fact was associated with a much faster and more complete recovery (Wiesel and Hubel, 1965a; Wiesel and Hubel, 1965b). Furthermore, these experiments led rapidly to the concept of the critical or sensitive period for plasticity, as it was found that the timing of deprivation, in terms of both onset and duration, was important for dictating the extent of deficits seen (Hubel and Wiesel, 1970; Daw et al., 1978; Teller and Boothe, 1979; Olson and Freeman, 1980). These findings led directly to the current clinical practice of diagnosing and treating human amblyopia as early in life as possible (Mitchell and MacKinnon, 2002).

In the current project, I aimed to bridge the gap between two different fields of amblyopia research. First, the animal studies in the tradition of Hubel and Wiesel have taught us much 
about the mechanisms of cortical plasticity and amblyopia, but have largely been restricted to investigating young animals, in which the opportunities for plasticity are still optimal. In contrast, most studies of human amblyopia, especially using neuroimaging techniques, have focused on adults. This discrepancy has not been due to short-sightedness, as adults are typically more cooperative, easier to study, and able to give full consent for participation in fMRI studies. However, if we hope to use the knowledge we gain from studying human amblyopia to improve treatment, the next logical step is to refine and apply the techniques to children, who are most likely to benefit from these advances with their greater cortical plasticity.

Since amblyopia manifests in animal models as an anatomical defect of visual cortex organization, we aimed to characterize the organization of visual cortex in children with amblyopia. However, few techniques have been described for imaging individual ocular dominance columns in humans (Goodyear and Menon, 2001; Goodyear et al., 2002), and their results remain controversial, so I chose to study the larger organizational principle of retinotopy. To date, retinotopy has been well studied in adults (Sereno et al., 1995; Engel et al., 1997; Tootell et al., 1997), but not in any known study of children. Therefore, I began by studying retinotopic organization in children with normal vision, finding similar organization as in adults, except for measurably smaller extrastriate cortical areas in children (Chapter 3).

Next, I studied adults with amblyopia using similar retinotopic mapping techniques (Chapter 4). I found shifted cortical ocular dominance and reduced cortical binocularity, consistent with the animal literature. More significantly, I also found reduced representation of the central visual field of the amblyopic eye. This novel and provocative result has potential implications for monitoring and treating amblyopia, as it represents the first report of abnormal cortical organization in amblyopia using neuroimaging techniques. Finally, I studied a small group of children with amblyopia and found abnormal retinotopic organization in visual cortex which was similar to the pattern seen in amblyopic adults (Chapter 5).

The abnormal organization of visual cortex at the occipital pole found here in people with amblyopia is the most significant finding of the current project. Although it is possible that this visual field remapping is a reflection of abnormal thalamocortical projections, this seems unlikely since previous studies have found that the LGN is functionally normal in amblyopia. Furthermore, the normal spread of thalamocortical afferents to V1 is limited to about $2 \mathrm{~mm}$ 
(Ferster and LeVay, 1978), and would not be expected to sprout collateral projections over a far enough distance to account for the effect seen here.

Rather than a bottom-up explanation for this phenomenon, horizontal connections within V1 are more likely to underlie the visual field reorganization. Electrophysiological studies in cats and monkeys have shown that binocular retinal lesions placed to interrupt a specific visual field location result in silent regions within the LGN, but restored visual activity over large spans of visual cortex after several months of recovery (Darian-Smith and Gilbert, 1995). The neurons within the initially deprived cortex (termed the lesion projection zone, LPZ) learn to represent field locations adjacent to the retinal lesion, even though they can be located between 5 and 10 mm away from neurons normally corresponding to that receptive field (Kaas et al., 1990; Gilbert and Wiesel, 1992). Long-range horizontal connections span enough cortical distance to account for this effect (Gilbert, 1998; Levitt and Lund, 2002), and one recent study has shown that kainic acid injection in locations surrounding the deafferented cortex deactivates neurons at both the injection site and the corresponding location in the LPZ, implicating horizontal corticocortical connections as the key input to the LPZ (Calford et al., 2003).

In fact, it has also been shown using induced artificial scotomas that correlated firing increases in pairs of layer $2 / 3$ neurons separated by several millimeters in primary visual cortex, suggesting strengthened horizontal connections (Das and Gilbert, 1995). The mechanisms of this effect are still being explored, but probably reflect an altered balance of excitation and inhibition in these long-range lateral connections. Perhaps the loss of feedforward input to V1 under these conditions leads to less activity in local inhibitory circuits, unmasking excitatory input from horizontal connections (Hirsch and Gilbert, 1993). Some evidence suggests that these unmasked connections are further strengthened through the sprouting of new axonal boutons (Darian-Smith and Gilbert, 1994).

It is possible that similar mechanisms are involved in the visual field remapping found in this project. Under conditions of chronic input suppression of the weak eye in amblyopia, the lack of feedforward projections from input layers of $\mathrm{V} 1$ to layer $2 / 3$ could potentially lead to unmasking of lateral excitatory inputs. The mean visual field shift shown in the current study was approximately 2.5 degrees of visual angle in the adult amblyopes, much less than the nearly 10 degrees of remapping seen in earlier animal studies with retinal lesions (DeAngelis et al., 1994; Li and Li, 1994). Since amblyopia is a developmental disorder with its origins during 
peak plasticity in early life, it could be expected that even greater amounts of adaptive remapping might be possible.

Finally, it is also possible that the reorganization seen here has resulted from top-down feedback from extrastriate visual areas into foveal cortex. Several studies have found activation of visual cortex in blind subjects performing nonvisual tasks, such as Braille reading (Burton et al., 2002). It has even been suggested that this effect in blind subjects is subject to a critical period, with little cross-modal reorganization seen if the onset of blindness was later than 16 years old (Sadato et al., 2002). Therefore, it is likely that both horizontal and top-down inputs are contributing to the reorganization of visual cortex reported here.

These studies are novel because they represent the first known attempt to study retinotopic organization in adults and children with amblyopia. This is significant because retinotopic mapping is one of the primary fMRI techniques used by vision scientists for studying the visual brain. Future neuroimaging studies of amblyopia, especially in children, will be informed by and build upon the current study. 


\subsection{Comparisons with Previous Studies}

This section will address comparisons between aspects of the current study and those that have preceded it. We will deal first with comparisons to other neuroimaging studies in children (Chapter 3), and then move on to comparisons with other studies of amblyopia in adults (Chapter 4). Finally, we will briefly address the significance of our study of children with amblyopia with respect to Chapters 3 and 4, as well as the very limited external neuroimaging data available for amblyopic children (Chapter 5).

First, we must address the feasibility of comparing fMRI studies in children and adults (Chapter 3). The basic mechanism of the BOLD response in fMRI is known, but a detailed understanding of the coupling between changes in neural activity and changes in blood oxygenation and flow is still being developed (Logothetis, 2003; Logothetis and Wandell, 2004). Furthermore, we do not know precisely how this mechanism changes during the course of development. As noted in Chapters 3 and 5, early studies of the BOLD response in infants found negative signals, raising the possibility of dramatic developmental changes in the BOLD mechanism (Born et al., 1996; Yamada et al., 1997; Born et al., 1998). However, these effects were likely specific to the infant brain, as subsequent studies have reported more similar activation patterns in older children and adults (Martin et al., 1999; Kang et al., 2003; Wenger et al., 2004). In addition, a negative BOLD response was recently obtained in children and adults during slow wave sleep, as well as in some cases of sedated adults (Born et al., 2002a; Born et al., 2002b), suggesting that sleep or sedation may have been the key factor in these early studies.

As noted in Chapter 5, fMRI is now being used and widely accepted to investigate a wide

range of normal and abnormal developmental processes in children. This literature is rapidly growing, exploring such diverse topics in normal development as selective attention (Booth et al., 2003), language representation (Poldrack et al., 2001; Joseph et al., 2001; Booth et al., 2001; Booth et al., 2004; Wood et al., 2004), object recognition (Joseph and Farley, 2004; Gathers et al., 2004), and reward circuitry (May et al., 2004), as well as developmental pathologies such as attention-deficit hyperactivity disorder (Sowell et al., 2003; Booth et al., 2005), dyslexia (Temple et al., 2001; Temple et al., 2003), and autism (Brambilla et al., 2004). This widespread acceptance of fMRI techniques applied to children further supports our novel use of retinotopic mapping techniques which were developed in adults to pediatric populations. 
In our study of children with normal vision (Conner et al., 2004), we found no difference in cortical magnification functions between children and adults, and our values were highly consistent with those reported for adults in previous studies (Sereno et al., 1995; Engel et al., 1997). However, we did find smaller extrastriate cortical areas in children compared with adults. We obtained this result for both absolute size and percentage of the entire reconstructed neocortical sheet, suggesting that gross brain size is not a relevant factor, a finding consistent with previous literature indicating no significant change in cerebral volume after age 5 (Giedd et al., 1996; Reiss et al., 1996). Previous reports agree that extrastriate cortex may develop more slowly than striate cortex, showing that striate activity dominates the checkerboard-onset evoked potential of the children aged about 4-8 years, whereas extrastriate activity matures later in development (Ossenblok et al., 1992; Ossenblok et al., 1994). A few anatomical studies of children's brains also suggest a posterior-to-anterior maturation gradient (Garey, 1984; Sowell et al., 1999; Thompson et al., 2000), as does a recent VBM study which found reduced extrastriate gray matter in children but not adults with amblyopia, which also suggests a developmental delay in the maturation of these areas (Mendola et al., 2005).

In our next study (Chapter 4), we first found less activation in visual cortex for amblyopic eye stimulation compared with fellow or control eyes, consistent with many previous PET (Demer et al., 1988; Demer et al., 1997; Imamura et al., 1997; Choi et al., 2002; Mizoguchi et al., 2005) and fMRI reports (Barnes et al., 2001; Choi et al., 2001; Algaze et al., 2002; Lerner et al., 2003; Liu et al., 2004). Interestingly, human post-mortem investigations have not found evidence for abnormal anatomical ocular dominance column periodicity (Horton and Stryker, 1993; Horton and Hocking, 1996), but two recent fMRI studies have demonstrated functional ocular dominance shifts in V1 of human amblyopes, consistent with our results in V1 and V2 (Goodyear et al., 2002; Liu et al., 2004). Furthermore, a recent pair of fMRI papers have also shown reduced indices of cortical binocularity, similar to our findings (Lee et al., 2001; Algaze et al., 2002).

Most significantly, we reported the completely novel result of abnormal retinotopic organization in our adult amblyopes. We hypothesized that the central visual field deficit seen in amblyopia (Thomas, 1978; Katz et al., 1984) would be reflected as a decreased cortical representation of central field locations for the amblyopic eye, but did not fully expect the magnitude of shifted organization that we found. Rapid reorganization of visual cortex has been 
demonstrated in young animals after focal retinal (Chino et al., 1992; Chino et al., 1995) and cortical lesions (Eysel and Schweigart, 1999; Zepeda et al., 2003), but since amblyopia is a slowly developing disorder with no acute lesion, it would be difficult to precisely predict the outcome of the visual field mapping experiment. However, this new finding holds much promise as an objective marker of the degree of cortical receptive field mismatch between the amblyopic and fellow eyes, and could potentially be used to customize and monitor treatment in children and young adults.

Although this is the first demonstration of abnormal retinotopic organization in amblyopia, a few other studies have examined retinotopic organization in age-related macular degeneration (AMD). The fovea is usually most affected in AMD, resulting in a substantial central scotoma. Although AMD occurs late in life compared with amblyopia, it is a useful analog of the retinal lesion studies in animals discussed in the previous section. However, the results from these neuroimaging studies of AMD are mixed. The first study mapped retinotopy in one patient (60 years old) with recent-onset AMD (<3 years), and found a region of 'silent' visual cortex corresponding to the central scotoma (Sunness et al., 2004). In contrast, a later study found significant reorganization in two patients with much longer-term AMD (>20 years), with normally foveal cortex responding instead to more peripheral visual field locations (Baker et al., 2005). Clearly, the difference here seems to be the length of the deprivation, with cortical reorganization occurring only with years of abnormal visual experience. Interestingly, the effect seen in Baker et al. (2005) occurred in adults years past their visual critical periods, suggesting that some remapping is possible even after the critical period plasticity has waned.

Finally, our study of retinotopic organization in children with amblyopia is the first of its kind (Chapter 5), and is therefore difficult to compare the results with other previous studies. However, we did find a similar pattern of organization in these children (ages 10 to 14) to the pattern we found in amblyopic adults (I. Conner, J. Odom, T. Schwartz, and J. Mendola, submitted), suggesting that the visual system has reached functional maturity by this age, at least as is detectable using the current techniques. It will be important to try extending these techniques to younger children, who would be expected to have greater remaining plasticity and the greatest likelihood of benefit from these findings. 


\subsection{Directions for Future Study}

The most obvious direction for future study will be to extend these visual field mapping techniques to younger children in order to elucidate the timeline of the remapping phenomenon seen in adults (Chapter 4) and older children (Chapter 5). Ultimately, studying the postnatal development of the visual system as a model of plasticity using neuroimaging could provide as much information on a macroscopic level as animal studies have at the microscopic level. It would be fascinating to follow visual development longitudinally through the critical periods for orientation, ocular dominance, disparity tuning, etc. (Lewis and Maurer, 2005). Furthermore, the lessons from normal development could then be used to evaluate and tailor treatments designed to extend or reopen the critical periods.

Of course, a critical intermediate step might be to study the postnatal development of vision using fMRI in other species which are better characterized physiologically, like cats and monkeys, and then to correlate those findings with the known electrophysiology. Animal models have similar advantages in neuroimaging as in other fields, in that they can be used in more controlled experimental manipulations, including extremely high-field MR scanners capable of much more detailed spatial resolution than the clinical scanners available for use with human subjects.

Finally, it is important to note that the current study has only aimed to characterize the neurological impairment in amblyopia and to provide a set of tools for use in future studies. Much work remains to clarify the genetics and molecular biology of amblyopia, and then to develop more effective treatments for use in children and adults (Michaelides and Moore, 2004). Amblyopia is a complicated disorder, in which it can be difficult to separate the causes from the effects (Barrett et al., 2004). Early studies such as our investigation of the nature of amblyopic human brains will provide an initial baseline for evaluating future treatments (Bacal, 2004). 


\subsection{Conclusions}

In conclusion, we have measured retinotopic organization in children in multiple visual areas and in adults and children with amblyopia for the first time. In the process, we demonstrated the feasibility of applying retinotopic mapping techniques developed for adults in children, with only slight modifications. It will now be possible to compare retinotopic maps in normal children to children with visual disorders and monitor the effect of treatment variables over time, an approach that is already revealing neurological effects of remediation in children with dyslexia (Temple et al., 2003). In addition, we also demonstrated the use of monocular retinotopic mapping in amblyopia, integrating fixation and feedback response monitoring in order to assure adequate attention and stimulus delivery. As a result, we found evidence for cortical visual field remapping in amblyopia, an effect supported by the psychophysical literature but which had not been described using neuroimaging techniques. Finally, we began the work of applying fMRI techniques to children with amblyopia, in whom the benefits of knowledge resulting from these studies are most likely to accrue. Our results suggest that further study will be necessary in younger children, both with and without amblyopia, in order to better understand the neural mechanisms of human neural development, plasticity, and amblyopia. 


\subsection{References}

Algaze A, Roberts C, Leguire L, Schmalbrock P, Rogers G (2002) Functional magnetic resonance imaging as a tool for investigating amblyopia in the human visual cortex: a pilot study. J AAPOS 6:300-308.

Bacal DA (2004) Amblyopia treatment studies. Curr Opin Ophthalmol 15:432-436.

Baker CI, Peli E, Knouf N, Kanwisher NG (2005) Reorganization of visual processing in macular degeneration. J Neurosci 25:614-618.

Barnes GR, Hess RF, Dumoulin SO, Achtman RL, Pike GB (2001) The cortical deficit in humans with strabismic amblyopia. J Physiol 533:281-297.

Barrett BT, Bradley A, McGraw PV (2004) Understanding the neural basis of amblyopia. Neuroscientist 10:106-117.

Booth JR, Burman DD, Meyer JR, Gitelman DR, Parrish TB, Mesulam MM (2004) Development of brain mechanisms for processing orthographic and phonologic representations. J Cogn Neurosci 16:1234-1249.

Booth JR, Burman DD, Meyer JR, Lei Z, Trommer BL, Davenport ND, Li W, Parrish TB, Gitelman DR, Mesulam MM (2005) Larger deficits in brain networks for response inhibition than for visual selective attention in attention deficit hyperactivity disorder (ADHD). J Child Psychol Psychiatry 46:94-111.

Booth JR, Burman DD, Meyer JR, Lei Z, Trommer BL, Davenport ND, Li W, Parrish TB, Gitelman DR, Mesulam MM (2003) Neural development of selective attention and response inhibition. Neuroimage 20:737-751.

Booth JR, Burman DD, Van Santen FW, Harasaki Y, Gitelman DR, Parrish TB, Marsel Mesulam MM (2001) The development of specialized brain systems in reading and orallanguage. Neuropsychol Dev Cogn C Child Neuropsychol 7:119-141.

Born AP, Law I, Lund TE, Rostrup E, Hanson LG, Wildschiodtz G, Lou HC, Paulson OB (2002a) Cortical deactivation induced by visual stimulation in human slow-wave sleep. Neuroimage 17:1325-1335.

Born AP, Rostrup E, Miranda MJ, Larsson HB, Lou HC (2002b) Visual cortex reactivity in sedated children examined with perfusion MRI (FAIR). Magn Reson Imaging 20:199-205. 
Born P, Leth H, Miranda MJ, Rostrup E, Stensgaard A, Peitersen B, Larsson HB, Lou HC (1998) Visual activation in infants and young children studied by functional magnetic resonance imaging. Pediatr Res 44:578-583.

Born P, Rostrup E, Leth H, Peitersen B, Lou HC (1996) Change of visually induced cortical activation patterns during development. Lancet 347:543.

Brambilla P, Hardan AY, di Nemi SU, Caverzasi E, Soares JC, Perez J, Barale F (2004) The functional neuroanatomy of autism. Funct Neurol 19:9-17.

Burton H, Snyder AZ, Conturo TE, Akbudak E, Ollinger JM, Raichle ME (2002) Adaptive changes in early and late blind: a fMRI study of Braille reading. J Neurophysiol 87:589-607.

Calford MB, Wright LL, Metha AB, Taglianetti V (2003) Topographic plasticity in primary visual cortex is mediated by local corticocortical connections. J Neurosci 23:6434-6442.

Chino YM, Kaas JH, Smith EL, III, Langston AL, Cheng H (1992) Rapid reorganization of cortical maps in adult cats following restricted deafferentation in retina. Vision Res 32:789796.

Chino YM, Smith EL, III, Kaas JH, Sasaki Y, Cheng H (1995) Receptive-field properties of deafferentated visual cortical neurons after topographic map reorganization in adult cats. J Neurosci 15:2417-2433.

Choi MY, Lee DS, Hwang JM, Choi DG, Lee KM, Park KH, Yu YS (2002) Characteristics of glucose metabolism in the visual cortex of amblyopes using positron-emission tomography and statistical parametric mapping. J Pediatr Ophthalmol Strabismus 39:11-19.

Choi MY, Lee KM, Hwang JM, Choi DG, Lee DS, Park KH, Yu YS (2001) Comparison between anisometropic and strabismic amblyopia using functional magnetic resonance imaging. Br J Ophthalmol 85:1052-1056.

Chua B, Mitchell P (2004) Consequences of amblyopia on education, occupation, and long term vision loss. Br J Ophthalmol 88:1119-1121.

Conner IP, Sharma S, Lemieux SK, Mendola JD (2004) Retinotopic organization in children measured with fMRI. J Vis 4:509-523.

Darian-Smith C, Gilbert CD (1994) Axonal sprouting accompanies functional reorganization in adult cat striate cortex. Nature 368:737-740.

Darian-Smith C, Gilbert CD (1995) Topographic reorganization in the striate cortex of the adult cat and monkey is cortically mediated. J Neurosci 15:1631-1647. 
Das A, Gilbert CD (1995) Receptive field expansion in adult visual cortex is linked to dynamic changes in strength of cortical connections. J Neurophysiol 74:779-792.

Daw NW, Berman NE, Ariel M (1978) Interaction of critical periods in the visual cortex of kittens. Science 199:565-567.

DeAngelis GC, Freeman RD, Ohzawa I (1994) Length and width tuning of neurons in the cat's primary visual cortex. J Neurophysiol 71:347-374.

Demer JL, Grafton S, Marg E, Mazziotta JC, Nuwer M (1997) Positron-emission tomographic study of human amblyopia with use of defined visual stimuli. J AAPOS 1:158-171.

Demer JL, von Noorden GK, Volkow ND, Gould KL (1988) Imaging of cerebral blood flow and metabolism in amblyopia by positron emission tomography. Am J Ophthalmol 105:337-347.

Engel SA, Glover GH, Wandell BA (1997) Retinotopic organization in human visual cortex and the spatial precision of functional MRI. Cereb Cortex 7:181-192.

Eysel UT, Schweigart G (1999) Increased receptive field size in the surround of chronic lesions in the adult cat visual cortex. Cereb Cortex 9:101-109.

Ferster D, LeVay S (1978) The axonal arborizations of lateral geniculate neurons in the striate cortex of the cat. J Comp Neurol 182:923-944.

Flom MC, Neumaier RW (1966) Prevalence of amblyopia. Public Health Rep 81:329-341.

Garey LJ (1984) Structural development of the visual system of man. Hum Neurobiol 3:75-80.

Gathers AD, Bhatt R, Corbly CR, Farley AB, Joseph JE (2004) Developmental shifts in cortical loci for face and object recognition. Neuroreport 15:1549-1553.

Giedd JN, Snell JW, Lange N, Rajapakse JC, Casey BJ, Kozuch PL, Vaituzis AC, Vauss YC, Hamburger SD, Kaysen D, Rapoport JL (1996) Quantitative magnetic resonance imaging of human brain development: ages 4-18. Cereb Cortex 6:551-560.

Gilbert CD (1998) Adult cortical dynamics. Physiol Rev 78:467-485.

Gilbert CD, Wiesel TN (1992) Receptive field dynamics in adult primary visual cortex. Nature 356:150-152.

Goodyear BG, Menon RS (2001) Brief visual stimulation allows mapping of ocular dominance in visual cortex using fMRI. Hum Brain Mapp 14:210-217. 
Goodyear BG, Nicolle DA, Menon RS (2002) High resolution fMRI of ocular dominance columns within the visual cortex of human amblyopes. Strabismus 10:129-136.

Hillis A, Flynn JT, Hawkins BS (1983) The evolving concept of amblyopia: a challenge to epidemiologists. Am J Epidemiol 118:192-205.

Hirsch JA, Gilbert CD (1993) Long-term changes in synaptic strength along specific intrinsic pathways in the cat visual cortex. J Physiol 461:247-262.

Horton JC, Hocking DR (1996) Pattern of ocular dominance columns in human striate cortex in strabismic amblyopia. Vis Neurosci 13:787-795.

Horton JC, Stryker MP (1993) Amblyopia induced by anisometropia without shrinkage of ocular dominance columns in human striate cortex. Proc Natl Acad Sci U S A 90:5494-5498.

Hubel DH, Wiesel TN (1965) Binocular interaction in striate cortex of kittens reared with artificial squint. J Neurophysiol 28:1041-1059.

Hubel DH, Wiesel TN (1970) The period of susceptibility to the physiological effects of unilateral eye closure in kittens. J Physiol 206:419-436.

Hubel DH, Wiesel TN, LeVay S (1977) Plasticity of ocular dominance columns in monkey striate cortex. Philos Trans R Soc Lond B Biol Sci 278:377-409.

Imamura K, Richter H, Fischer H, Lennerstrand G, Franzen O, Rydberg A, Andersson J, Schneider H, Onoe H, Watanabe Y, Langstrom B (1997) Reduced activity in the extrastriate visual cortex of individuals with strabismic amblyopia. Neurosci Lett 225:173-176.

Joseph J, Noble K, Eden G (2001) The neurobiological basis of reading. J Learn Disabil 34:566579.

Joseph JE, Farley AB (2004) Cortical regions associated with different aspects of object recognition performance. Cogn Affect Behav Neurosci 4:364-378.

Kaas JH, Krubitzer LA, Chino YM, Langston AL, Polley EH, Blair N (1990) Reorganization of retinotopic cortical maps in adult mammals after lesions of the retina. Science 248:229-231.

Kang HC, Burgund ED, Lugar HM, Petersen SE, Schlaggar BL (2003) Comparison of functional activation foci in children and adults using a common stereotactic space. Neuroimage 19:1628.

Katz LM, Levi DM, Bedell HE (1984) Central and peripheral contrast sensitivity in amblyopia with varying field size. Doc Ophthalmol 58:351-373. 
Lee KM, Lee SH, Kim NY, Kim CY, Sohn JW, Choi MY, Gyu CD, Hwang JM, Ho PK, Lee DS, Suk YY, Hyun CK (2001) Binocularity and spatial frequency dependence of calcarine activation in two types of amblyopia. Neurosci Res 40:147-153.

Lerner Y, Pianka P, Azmon B, Leiba H, Stolovitch C, Loewenstein A, Harel M, Hendler T, Malach R (2003) Area-specific amblyopic effects in human occipitotemporal object representations. Neuron 40:1023-1029.

Levitt JB, Lund JS (2002) The spatial extent over which neurons in macaque striate cortex pool visual signals. Vis Neurosci 19:439-452.

Lewis TL, Maurer D (2005) Multiple sensitive periods in human visual development: Evidence from visually deprived children. Dev Psychobiol 46:163-183.

Li CY, Li W (1994) Extensive integration field beyond the classical receptive field of cat's striate cortical neurons--classification and tuning properties. Vision Res 34:2337-2355.

Liu GT, Miki A, Francis E, Quinn GE, Modestino EJ, Bonhomme GR, Haselgrove JC (2004) Eye dominance in visual cortex in amblyopia using functional magnetic resonance imaging. $\mathrm{J}$ AAPOS 8:184-186.

Logothetis NK (2003) The underpinnings of the BOLD functional magnetic resonance imaging signal. J Neurosci 23:3963-3971.

Logothetis NK, Wandell BA (2004) Interpreting the BOLD signal. Annu Rev Physiol 66:735769.

Martin E, Joeri P, Loenneker T, Ekatodramis D, Vitacco D, Hennig J, Marcar VL (1999) Visual processing in infants and children studied using functional MRI. Pediatr Res 46:135-140.

May JC, Delgado MR, Dahl RE, Stenger VA, Ryan ND, Fiez JA, Carter CS (2004) Event-related functional magnetic resonance imaging of reward-related brain circuitry in children and adolescents. Biol Psychiatry 55:359-366.

Mendola JD, Conner IP, Roy A, Chan ST, Schwartz TL, Odom JV, Kwong KK (2005) Voxelbased analysis of MRI detects abnormal visual cortex in children and adults with amblyopia. Hum Brain Mapp 25:222-236.

Michaelides M, Moore AT (2004) The genetics of strabismus. J Med Genet 41:641-646.

Mitchell DE, MacKinnon S (2002) The present and potential impact of research on animal models for clinical treatment of stimulus deprivation amblyopia. Clin Exp Optom 85:5-18. 
Mizoguchi S, Suzuki Y, Kiyosawa M, Mochizuki M, Ishii K (2005) Differential activation of cerebral blood flow by stimulating amblyopic and fellow eye. Graefes Arch Clin Exp Ophthalmol.

Ohlsson J, Villarreal G, Sjostrom A, Abrahamsson M, Sjostrand J (2001) Visual acuity, residual amblyopia and ocular pathology in a screened population of 12-13-year-old children in Sweden. Acta Ophthalmol Scand 79:589-595.

Ohlsson J, Villarreal G, Sjostrom A, Cavazos H, Abrahamsson M, Sjostrand J (2003) Visual acuity, amblyopia, and ocular pathology in 12- to 13-year-old children in Northern Mexico. J AAPOS 7:47-53.

Olson CR, Freeman RD (1980) Profile of the sensitive period for monocular deprivation in kittens. Exp Brain Res 39:17-21.

Ossenblok P, Reits D, Spekreijse H (1992) Analysis of striate activity underlying the pattern onset EP of children. Vision Res 32:1829-1835.

Ossenblok P, Reits D, Spekreijse H (1994) Check size dependency of the sources of the hemifield-onset evoked potential. Doc Ophthalmol 88:77-88.

Poldrack RA, Temple E, Protopapas A, Nagarajan S, Tallal P, Merzenich M, Gabrieli JD (2001) Relations between the neural bases of dynamic auditory processing and phonological processing: evidence from fMRI. J Cogn Neurosci 13:687-697.

Reiss AL, Abrams MT, Singer HS, Ross JL, Denckla MB (1996) Brain development, gender and IQ in children. A volumetric imaging study. Brain 119 ( Pt 5):1763-1774.

Sadato N, Okada T, Honda M, Yonekura Y (2002) Critical period for cross-modal plasticity in blind humans: a functional MRI study. Neuroimage 16:389-400.

Sereno MI, Dale AM, Reppas JB, Kwong KK, Belliveau JW, Brady TJ, Rosen BR, Tootell RB (1995) Borders of multiple visual areas in humans revealed by functional magnetic resonance imaging. Science 268:889-893.

Sowell ER, Thompson PM, Holmes CJ, Jernigan TL, Toga AW (1999) In vivo evidence for post-adolescent brain maturation in frontal and striatal regions. Nat Neurosci 2:859-861.

Sowell ER, Thompson PM, Welcome SE, Henkenius AL, Toga AW, Peterson BS (2003) Cortical abnormalities in children and adolescents with attention-deficit hyperactivity disorder. Lancet 362:1699-1707. 
Sunness JS, Liu T, Yantis S (2004) Retinotopic mapping of the visual cortex using functional magnetic resonance imaging in a patient with central scotomas from atrophic macular degeneration. Ophthalmology 111:1595-1598.

Teller DY, Boothe R (1979) Development of vision in infant primates. Trans Ophthalmol Soc U K 99:333-337.

Temple E, Deutsch GK, Poldrack RA, Miller SL, Tallal P, Merzenich MM, Gabrieli JD (2003) Neural deficits in children with dyslexia ameliorated by behavioral remediation: evidence from functional MRI. Proc Natl Acad Sci U S A 100:2860-2865.

Temple E, Poldrack RA, Salidis J, Deutsch GK, Tallal P, Merzenich MM, Gabrieli JD (2001) Disrupted neural responses to phonological and orthographic processing in dyslexic children: an fMRI study. Neuroreport 12:299-307.

Thomas J (1978) Normal and amblyopic contrast sensitivity function in central and peripheral retinas. Invest Ophthalmol Vis Sci 17:746-753.

Thompson PM, Giedd JN, Woods RP, MacDonald D, Evans AC, Toga AW (2000) Growth patterns in the developing brain detected by using continuum mechanical tensor maps. Nature 404:190-193.

Tommila V, Tarkkanen A (1981) Incidence of loss of vision in the healthy eye in amblyopia. $\mathrm{Br}$ J Ophthalmol 65:575-577.

Tootell RB, Mendola JD, Hadjikhani NK, Ledden PJ, Liu AK, Reppas JB, Sereno MI, Dale AM (1997) Functional analysis of V3A and related areas in human visual cortex. J Neurosci 17:7060-7078.

von Noorden GK, Campos EC (2001) Binocular vision and ocular motility: Theory and management of strabismus. St. Louis: Mosby.

Wenger KK, Visscher KM, Miezin FM, Petersen SE, Schlaggar BL (2004) Comparison of sustained and transient activity in children and adults using a mixed blocked/event-related fMRI design. Neuroimage 22:975-985.

Wiesel TN, Hubel DH (1963) Single-cell responses in striate cortex of kittens deprived of vision in one eye. J Neurophysiol 26:1003-1017.

Wiesel TN, Hubel DH (1965a) Comparison of the effects of unilateral and bilateral eye closure on cortical unit responses in kittens. J Neurophysiol 28:1029-1040. 
Wiesel TN, Hubel DH (1965b) Extent of recovery from the effects of visual deprivation in kittens. J Neurophysiol 28:1060-1072.

Wood AG, Harvey AS, Wellard RM, Abbott DF, Anderson V, Kean M, Saling MM, Jackson GD (2004) Language cortex activation in normal children. Neurology 63:1035-1044.

Yamada H, Sadato N, Konishi Y, Kimura K, Tanaka M, Yonekura Y, Ishii Y (1997) A rapid brain metabolic change in infants detected by fMRI. Neuroreport 8:3775-3778.

Zepeda A, Vaca L, Arias C, Sengpiel F (2003) Reorganization of visual cortical maps after focal ischemic lesions. J Cereb Blood Flow Metab 23:811-820. 


\section{Curriculum Vitae}




\author{
IAN P. CONNER \\ 736 Clinton Avenue \\ Morgantown, WV 26505 \\ (304) 319-1158 \\ iconner@mix.wvu.edu
}

\title{
EDUCATION:
}

2005-2007 M.D. Clinical Training Curriculum

West Virginia University School of Medicine, Morgantown, WV

2000-2005 Ph.D. in Anatomy and Neurobiology

Dissertation Project: fMRI Studies of Amblyopia: Pediatric and Adult Perspectives

Dissertation Advisor: Janine D. Mendola, Ph.D.

WVU School of Medicine, Morgantown, WV

1998-2000 M.D. Basic Sciences Curriculum

WVU School of Medicine, Morgantown, WV

1994-1998 B.S. in Chemical Engineering

WVU College of Engineering and Mineral Resources, Morgantown, WV

\section{RESEARCH EXPERIENCE:}

2000-2005 Graduate Student, Department of Neurobiology and Anatomy

WVU School of Medicine, Morgantown, WV

1996 NSF-Research Experience for Undergraduates Fellow

Summer Project: Endothelial Cell Adhesion Properties on Synthetic Polymer Surfaces

Project Advisor: William Wagner, Ph.D.

University of Pittsburgh, Pittsburgh, PA

\section{TEACHING EXPERIENCE:}

2001 Teaching Assistant, Medical Neurobiology, Department of Neurobiology and Anatomy Taught gross neuroanatomy (including dissection) and histology to medical students WVU School of Medicine, Morgantown, WV

2000 Instructor, Kaplan MCAT Preparation Course

Taught physical and biological sciences to pre-medical undergraduate students

West Virginia Junior College, Morgantown, WV

1995-1996 Teaching Assistant, WVU Math Workshop

Taught and tutored college algebra to undergraduate students

WVU Eberly College of Arts and Sciences, Morgantown, WV

\section{HONORS AND AWARDS:}

1998 WVU Honors and Summa Cum Laude Graduate

1994-1998 WVU Honors Program

1994-1998 West Virginia University Presidential Scholarship 


\section{PAST RESEARCH AWARDS:}

2005 Selected for Talk Session at the Van Liere Convocation and Research Day

WVU Health Sciences Center, Morgantown, WV

2003 Student Travel Fellowship

Organization for Human Brain Mapping Annual Meeting, New York, NY

1998 Outstanding Student Researcher

WVU Honors Program, Morgantown, WV

\section{MEMBERSHIP IN PROFESSIONAL SOCIETIES:}

2002-2003, 2005 Vision Sciences Society

2000, 2003 Organization for Human Brain Mapping

2001 Cognitive Neurosciences Society

1998-2005 American Medical Association

1998-2005 American Medical Student Association

1998-2005 American Academy of Family Physicians

1996-1998 American Institute of Chemical Engineers

\section{PUBLICATIONS:}

Mendola JD, Conner IP, Roy A, Chan ST, Schwartz TL, Odom JV, Kwong KK (2005). Voxelbased analysis of MRI detects abnormal visual cortex in children and adults with amblyopia. Hum Brain Mapp 25:222-236.

Conner IP, Sharma S, Lemieux SK, Mendola JD (2004). Retinotopic organization in children measured with fMRI. $J$ Vis 4:509-523.

Conner IP, Odom JV, Schwartz TL, Mendola JD. Monocular representation in visual cortex of amblyopic adults measured with fMRI (submitted to Cereb Cortex).

Agrawal R, Conner IP, Odom JV, Schwartz TL, Mendola JD. Relating binocular and monocular vision in strabismic and anisometropic amblyopia (submitted to Invest Ophthalmol Vis Sci).

Mendola JD, Conner IP, Sharma S, Lemieux SK. fMRI measures of visible and invisible shapes in human visual cortex: a filling-in paradigm (submitted to $J$ Cog Neurosci).

Conner IP, Schwartz TL, Odom JV, Mendola JD. Monocular representation in visual cortex of amblyopic children measured with fMRI (in preparation).

Mendola JD, Conner IP. Does ocular dominance predict fMRI signals in retinotopic cortex? (in preparation). 


\section{PUBLISHED ABSTRACTS AND PRESENTATIONS:}

Conner IP (2005). fMRI studies of amblyopia: pediatric and adult perspectives [Oral Presentation]. Dissertation Seminar, WVU School of Medicine, Morgantown, WV.

Conner IP, Mendola JD (2005). What does an amblyopic eye tell human visual cortex? Vision Sciences Society Meeting, Sarasota, FL.

Mendola JD, Conner IP (2005). Does eye dominance predict fMRI signals in retinotopic cortex? Vision Sciences Society Meeting, Sarasota, FL.

Conner IP, Mendola JD (2005). What does an amblyopic eye tell human visual cortex [Oral Presentation]? Van Liere Convocation and Research Day, WVU Health Sciences Center, Morgantown, $W V$.

Conner IP, Mendola JD (2005). What does an amblyopic eye tell human visual cortex? WVU Sigma Xi Research Day, Morgantown, WV.

Mendola JD, Chan ST, Conner IP, Roy A, Schwartz TL, Odom JV, Kwong KK (2004). Loss of visual cortex in amblyopia correlates with perception. Organization for Human Brain Mapping Meeting, Budapest, Hungary.

Mendola JD, Chan ST, Roy A, Conner IP, Schwartz TL, Odom JV, Kwong KK (2004). Loss of visual cortex in children and adults with amblyopia. Vision Sciences Society Meeting, Sarasota, FL.

Conner IP, Schwartz TL, Odom JV, Mendola JD (2003). Abnormal maps of retinotopic space in amblyopia adults. Organization for Human Brain Mapping Meeting, New York, NY.

Sharma S, Trimmier J, Roy A, Schwab G, Upadhyay J, Conner IP, Mendola JD (2003). The functional anatomy of the contrast sensitivity function. Organization for Human Brain Mapping Meeting, New York, NY.

Conner IP, Schwartz TL, Odom JV, Mendola JD (2003). Monocular retinotopic mapping in amblyopic adults [Oral Presentation]. Vision Sciences Society Meeting, Sarasota, FL.

Conner IP (2002). Investigation of amblyopic visual perception in humans using fMRI [Oral Presentation]. Department of Neurobiology and Anatomy Seminar Series, WVU School of Medicine, Morgantown, WV.

Mendola JD, Roy A, Conner IP, Chan ST, Kwong KK, Fischl B (2002). Cortical morphological changes characterize anisometropic and strabismic amblyopia. Organization for Human Brain Mapping Meeting, Sendai, Japan. 
Marano GD, Roy A, Conner IP, Lemieux SK, Gocke R (2002). Gadolinium-based MR contrast enhancement effects on unfolding and thickness measurement of the human cortex. Organization for Human Brain Mapping Meeting, Sendai, Japan.

Conner IP, Sharma S, Mendola JD (2002). Retinotopic mapping in children with normal vision. Vision Sciences Society Meeting, Sarasota, FL.

Conner IP, Sharma S, Mendola JD (2002). Retinotopic mapping in children with normal vision. Van Liere Convocation and Research Day, WVU Health Sciences Center, Morgantown, WV.

Conner IP (2001). Using functional neuroimaging to study visual deficits in amblyopia [Oral Presentation]. Department of Neurobiology and Anatomy Seminar Series, WVU School of Medicine, Morgantown, WV.

Mendola JD, Conner IP, Sharma S, Lemieux SK (2001). fMRI measures of visible and invisible shapes in human visual cortex: a filling-in paradigm. Society for Neuroscience Meeting, San Diego, CA. 